
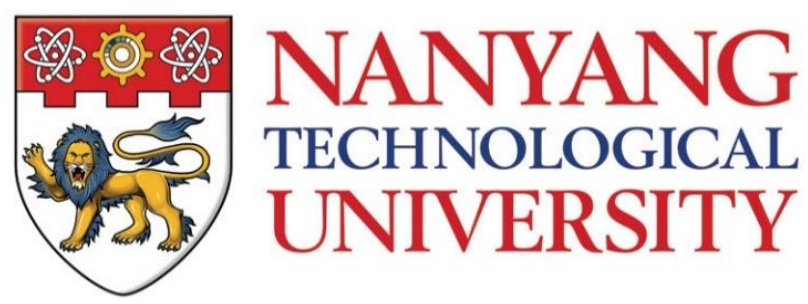

\title{
EFFECTS OF SURFACE FUNCTIONALIZATION AND PHYSICAL CONSTRAINTS OF POLY (DIMETHYLSILOXANE) ON CELLULAR BEHAVIORS
}

SHREYAS KUDDANNAYA

SCHOOL OF MECHANICAL AND AEROSPACE ENGINEERING 


\section{EFFECTS OF SURFACE FUNCTIONALIZATION AND PHYSICAL CONSTRAINTS OF POLY (DIMETHYLSILOXANE) ON CELLULAR BEHAVIORS}

SHREYAS KUDDANNAYA

School of Mechanical and Aerospace Engineering

A thesis submitted to the Nanyang Technological University in partial fulfillment of the requirement for the degree of Doctor of Philosophy 


\title{
Acknowledgement
}

\author{
"A journey of thousand miles begins with a single step" \\ -Lao Tzu (Tao Te Ching, chapter 64)
}

First and foremost, I would like to express my sincere gratitude to Assistant Professor Yilei Zhang for providing me an opportunity to realize my research pursuits and for constantly guiding me throughout the $\mathrm{PhD}$ thesis project. I thank him for his unwavering support with framing research questions, shaping novel ideas and helping me with fruitful collaborations.

A special thanks to Prof. Yuejun Kang for his timely inputs on my collaborated project and for providing access to the facilities at the AML lab and cleanroom in SCBE. I am also grateful to Dr. Yon Jin Chuah and Dr. Nishanth Menon for their collaborative support in my projects. I thank Asst. Prof Yeong Wai Yee and Asst. Prof Xu Chenjie for their support as thesis advisors. I would like to thank the research fellows Dr. Yantao Fan, Dr. Jin Lin, Dr. Sunanda Roy, Dr. Weihong Long and Dr. Sisi Li for their support with several fruitful research ideas and tasks.

I thank Ms. Heng Chee Hoon for her persistent help with the lab matters and purchases. I am very grateful for my friends and lab mates especially Qinwei Xu, Zhengkun Yi, Li Cheng, Alain Phuoc, Andy Liew, Vivek Damodar and Sathya Bhaskar for the great moments we shared both within and outside the lab.

My parents, grandmother and brother have been a constant source of encouragement for my studies and research. I am forever grateful for their love and blessings. My wife Abhijnha has been a pillar of support throughout my studies. I am thankful for her help and patience. Finally, I would like to extend my gratitude to the School of Mechanical and Aerospace Engineering, NTU for supporting my study. 


\section{Table of Contents}

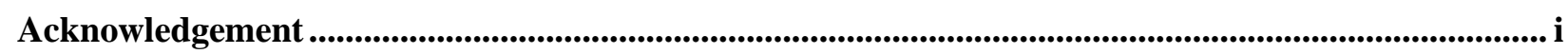

List of figures.......................................................................................................................................................... vii

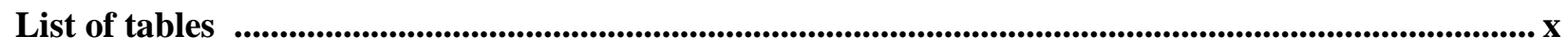

Abbreviations .................................................................................................................................................................

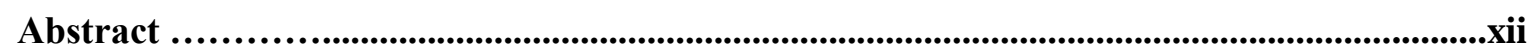

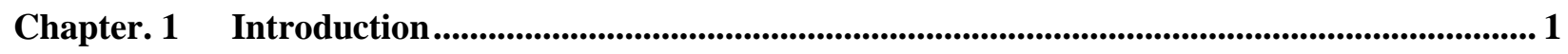

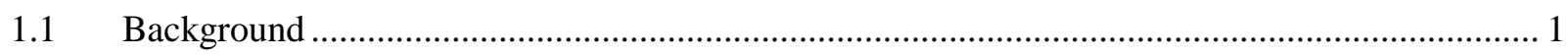

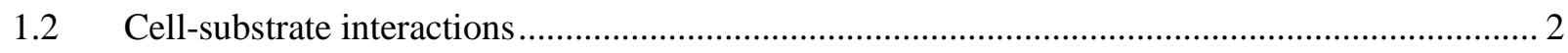

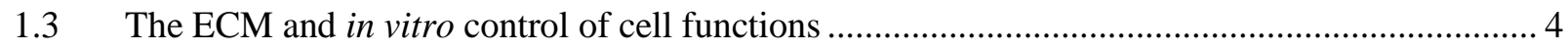

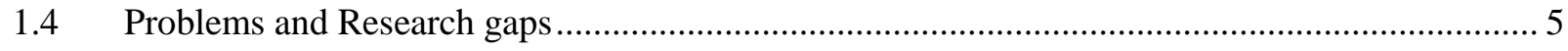

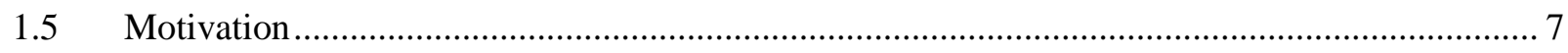

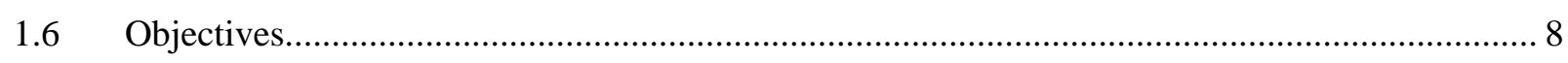

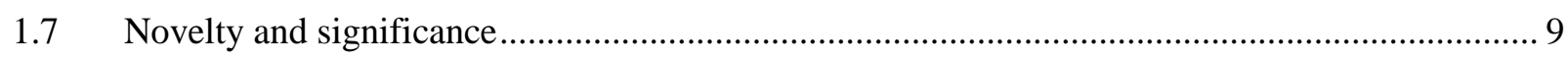

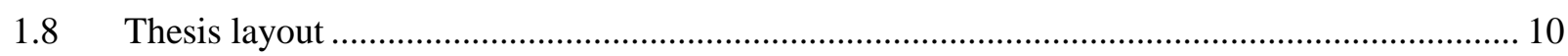

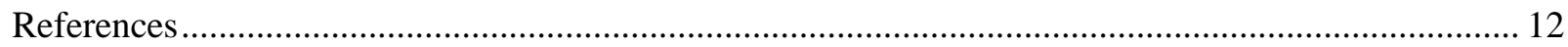

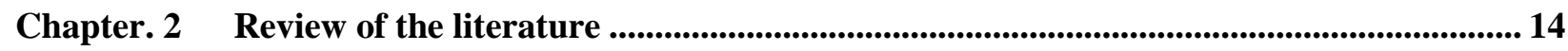

2.1 Mammalian cell differentiation and development ............................................................... 14

2.2 Cell microenvironments and Cell-ECM relationships ........................................................ 16

2.3 Impact of ECM composition and properties on cell behaviors ............................................ 17

2.4 Regulatory role of Integrin-ECM composition cytoskeleton crosstalk................................... 19

2.5 Engineering cells and cell environments for regenerative therapies ....................................... 21

2.6 hMSCs in tissue engineering and role of stem cell niches ................................................. 22

2.6.1 Cell-matrix relationships in directing hMSC cell behaviors .......................................... 23

$2.7 \quad$ Neural development, neurogenesis and neuronal niches ................................................ 24

2.7.1 Cell-matrix relationships in directing neuronal cell behaviors ....................................... 26

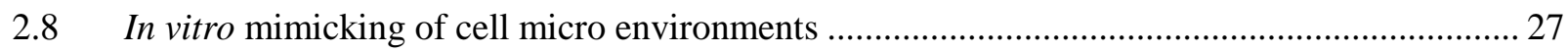

2.8.1 Rationale for in vitro biomimicking for regenerative and developmental research........... 27

2.8.2 In vitro manipulation of ECM: Overview and recent trends ............................................ 29

Control of biochemical and biophysical environments ....................................................... 29

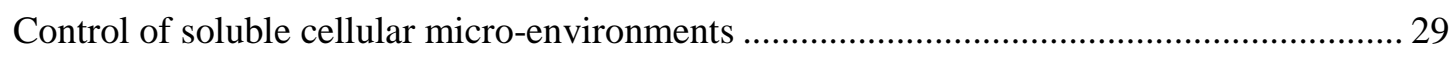

2.8.3 Role of Surface chemistry and ECM protein in modulating cell behaviors....................... 30

2.8.3.1 Surface chemistry/ECM studies on hMSC and hMSC-like cells .................................. 33

2.8.3.2 Surface chemistry/ECM based platforms for neuronal cell investigations .................... 36 
2.8.3.3 Traditional v/s SAM based approaches for ECM protein immobilization...................... 38

2.8.4 Role of ECM topography and mechanics in controlling key cell behaviors ...................... 41

2.8.4.1 Substrate topography and hMSC behaviors .............................................................. 42

2.8.4.2 Substrate topography and Neuronal cell behaviors.................................................... 43

2.9 Design of in vitro platforms for study of specific cell functions .............................................. 46

2.9.1 Differentiation/Tissue/Organ on-a-chip systems ....................................................... 47

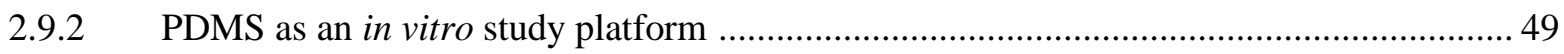

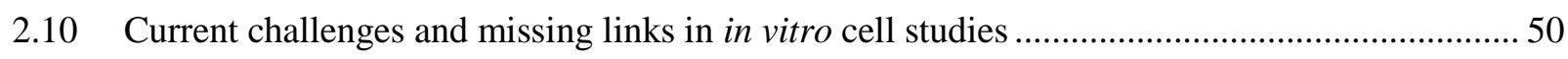

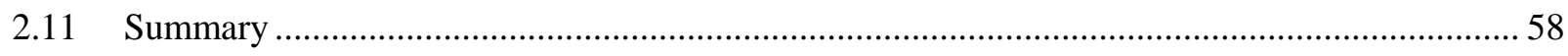

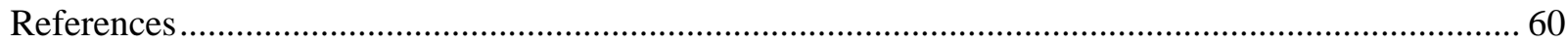

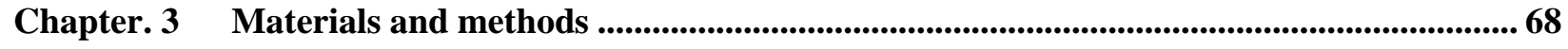

3.1 Substrate preparation, modification and microfabrication ................................................... 68

3.1.1 PDMS preparation and surface chemical modification................................................ 68

3.1.2 Fabrication of topographic micro-features by Photo-lithography ..................................... 69

3.1.3 Fabrication of Hole-pillar and groove micro-features for hMSC cell studies................... 70

3.1.4 Fabrication of geometrical micro-trap shape array for neuronal cell studies.................... 72

3.1.5 Fabrication and modification of PDMS microchannels for hMSC/neuron study .............. 73

3.2 Characterization of substrate chemical and topographical properties.................................... 74

3.2.1 Characterization of Surface's Hydrophilic Properties .................................................. 74

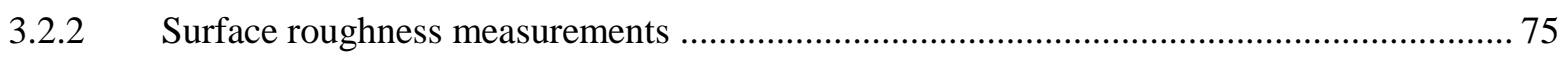

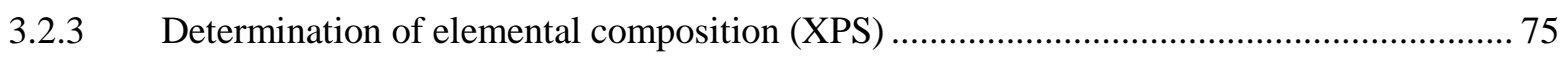

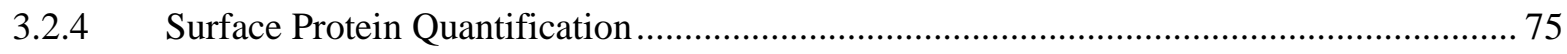

3.2.5 Surface profile analysis by profiler and SEM ............................................................. 76

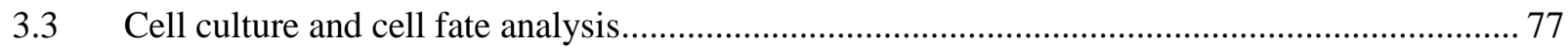

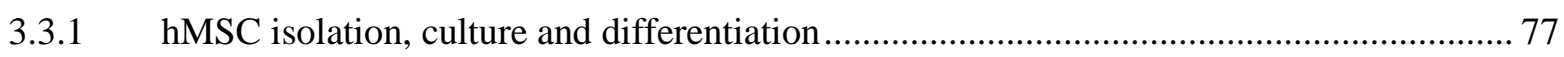

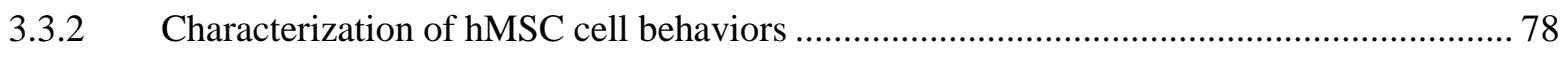

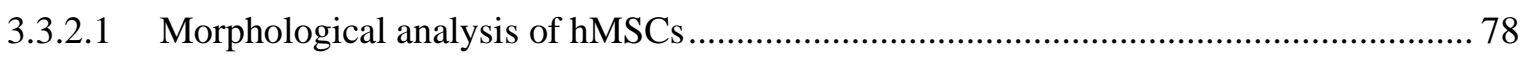

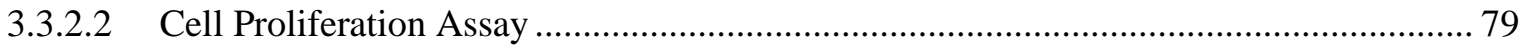

3.3.2.3 Characterization of Cell Adhesion ........................................................................... 79

3.3.2.4 Histological staining and bio-mineralization analysis ............................................. 80

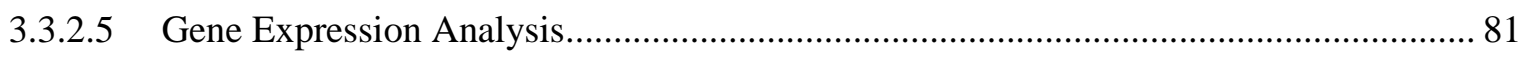

3.3.3 Isolation and culture of nascent cortical neurons ........................................................ 82

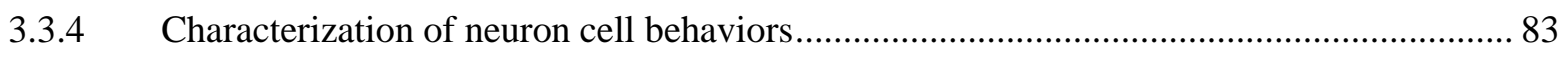

3.3.4.1 Immunostaining with neuronal and glial cell markers................................................. 83

3.3.4.2 Neurite Analysis on chemically modified flat substrates ............................................. 83

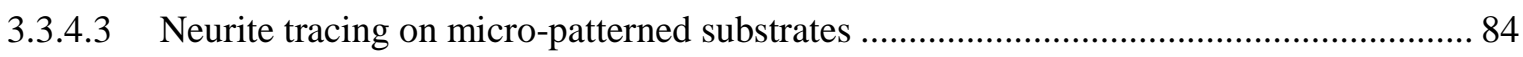




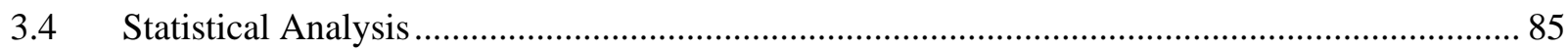

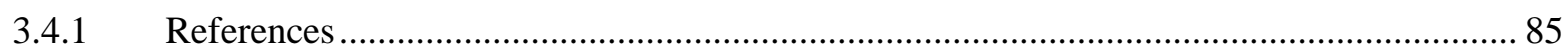

Chapter. 4 Effects of surface chemical modification in regulating hMSC behaviors \& potency $\quad 86$

4.1 Enhanced adhesion and proliferation of hMSCs on covalently crosslinked ECM protein

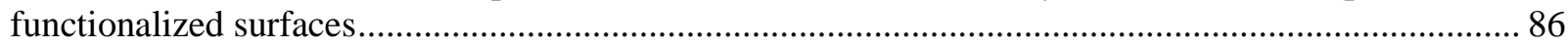

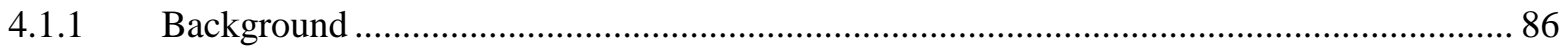

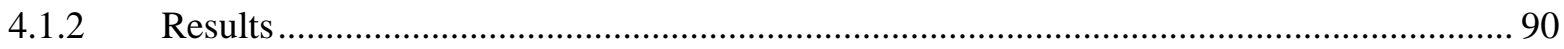

4.1.2.1 X-ray Photoelectron Spectroscopy (XPS) .................................................................. 90

4.1.2.2 Surface wettability of modified/unmodified PDMS surfaces ....................................... 91

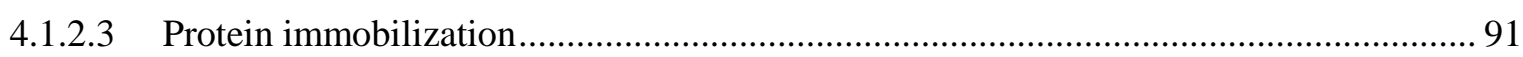

4.1.2.4 Characterization of cell adhesion on modified/unmodified PDMS surfaces ................. 92

4.1.2.5 Proliferation of cells on different chemically modified/unmodified PDMS surfaces 94

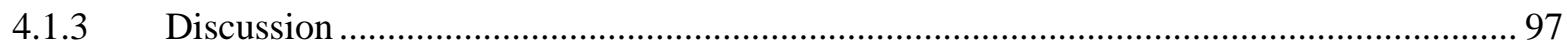

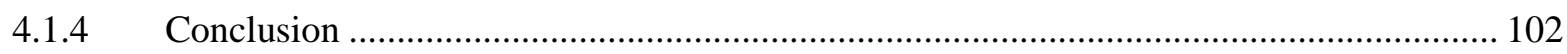



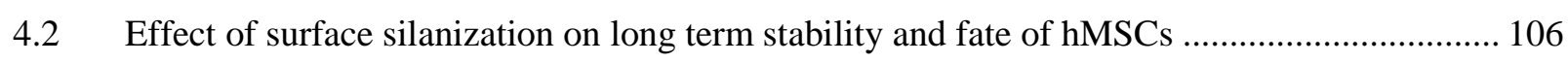

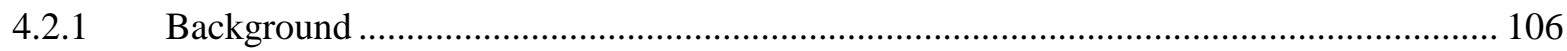

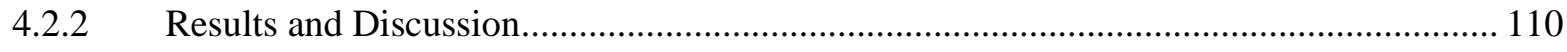

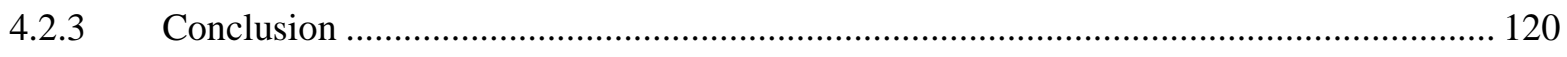

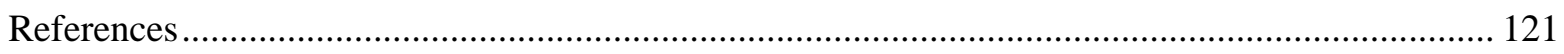

4.3 Effect of silane type, structure and functionality on hMSC fates: miniaturized model to evaluate cell-surface chemical interactions on PDMS microchip................................................... 123

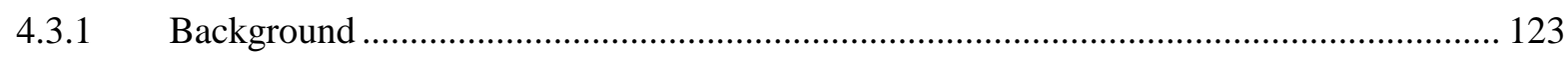

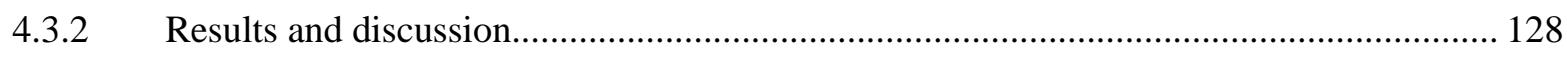

4.3.2.1 Surface hydrophobicity, Roughness and protein retention ........................................ 128

4.3.2.2 Cell morphology, initial adhesion and surface coverage (D14) ................................ 131

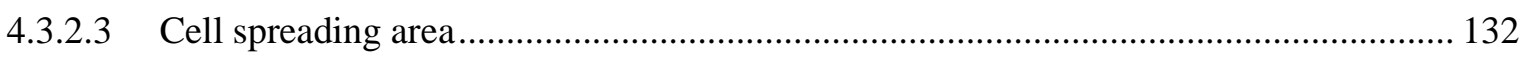

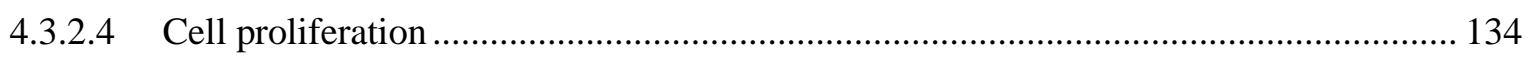

4.3.2.5 Adhesion and morphology within silanized micro-channels .................................... 135

4.3.2.6 Biomineralization and osteogenic biomarker expression......................................... 137

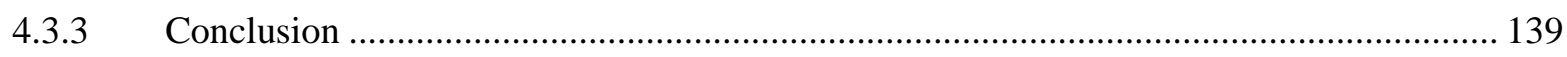

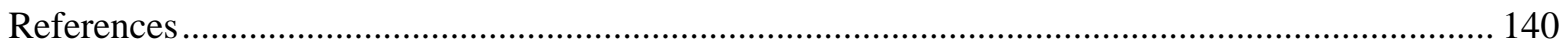

Chapter. 5 Effect of surface chemistry on neuronal cell behaviors ................................................ 143

5.1 Enhanced In-vitro Biocompatibility of Chemically Modified PDMS surfaces for Stable Adhesion and Long-term Investigation of Brain Cerebral Cortex Cells ............................................ 143

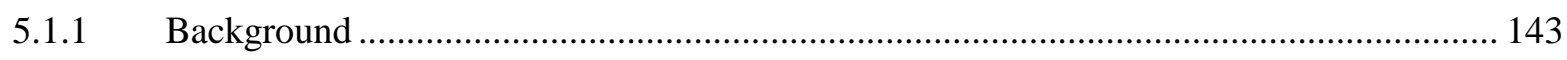




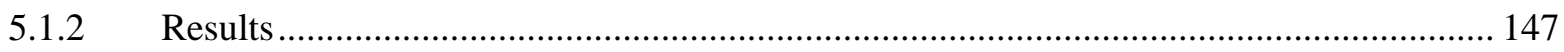

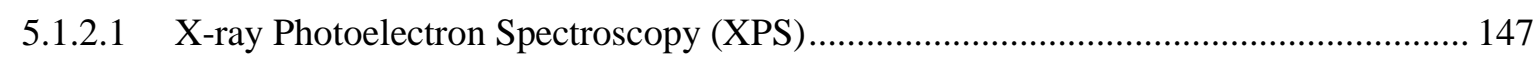

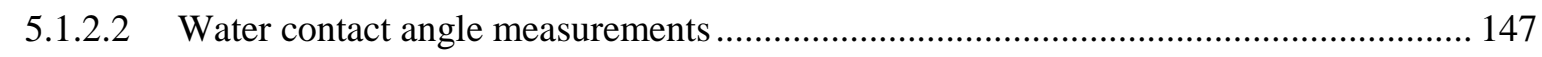

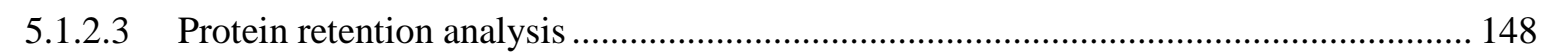

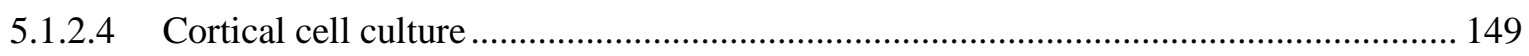

5.1.2.5 Mean neurite length and primary branching ...................................................... 150

5.1.2.6 Immunostaining and Scanning Electron Microscopy ............................................. 152

5.1.2.7 Long term culture within PDMS microfluidic chip ................................................. 154

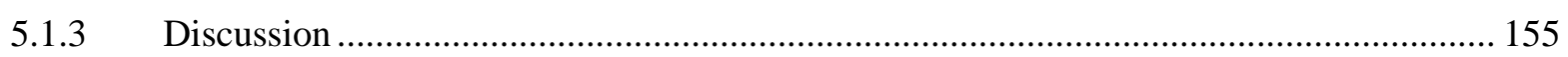

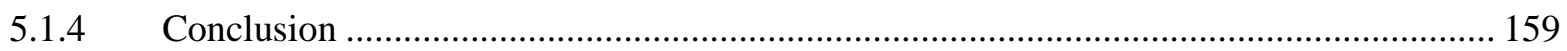

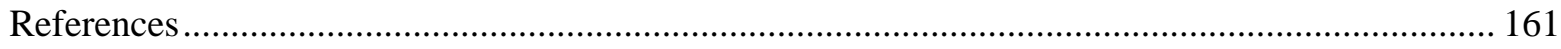

Chapter. 6 Effect of inclusion of topographic elements on hMSC behaviors ................................ 166

6.1 Combined effects of multi-topographical and surface chemical environment on

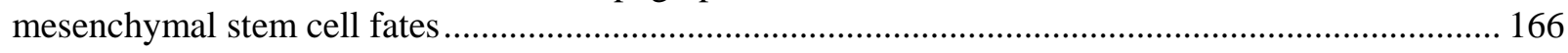

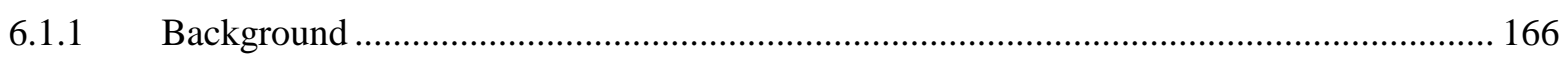

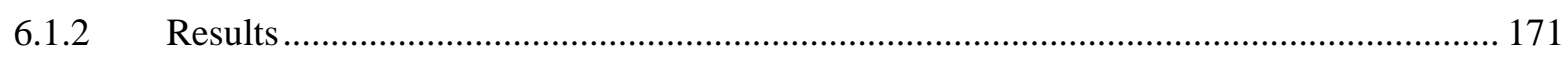

6.1.2.1 Cytoskeletal profile and surface coverage of adherent cell layers ............................... 171

6.1.2.2 Morphological analysis of hMSCs on topographic substrates ................................... 173



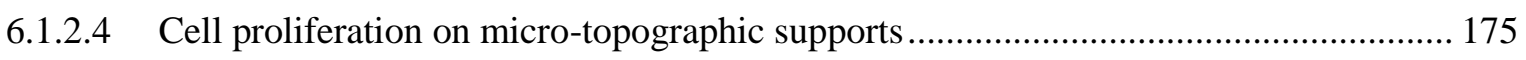

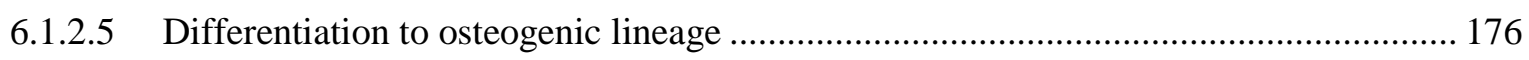

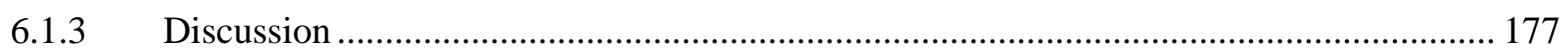

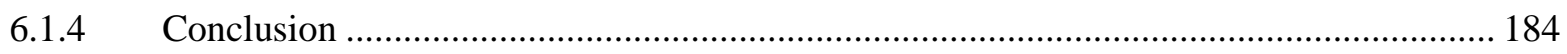

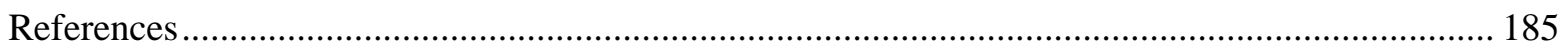

Chapter. 7 Effect of substrate topography on neuronal cell behaviors......................................... 189

7.1 Geometrically mediated topographic steering of neurite behaviors and network

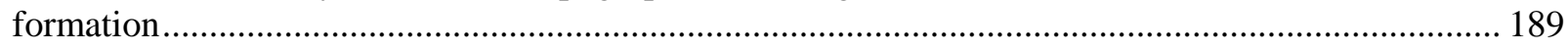

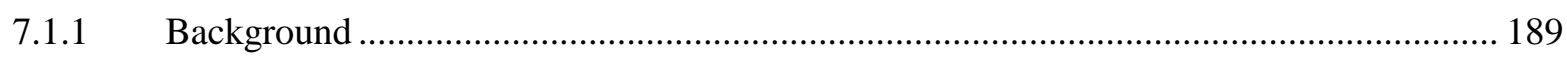

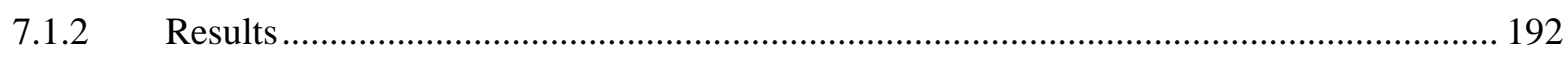

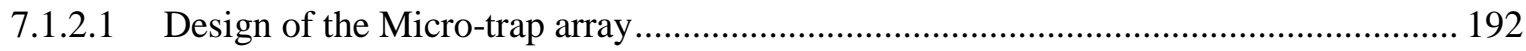

7.1.2.2 Neuronal growth and neurite deflection analysis .................................................... 193

7.1.2.3 Morphological analysis of entrapped neurons ......................................................... 195

Analysis of cell cytoskeleton and neurite branching points ............................................... 196

Analysis of F-actin profiles along angular/curved cues ..................................................... 197

Microtrap geometry affects neurite branching and length ............................................... 199

7.1.2.4 External guidance of neurites and the formation of minimalistic neuron networks...... 200

Neurite pathfinding along external cues and the influence of glial cells .............................. 201 
Analysis of Minimalistic network formation on patterned substrates ................................. 202

Regulation of neurite directionality and length in minimalistic networks ............................ 204

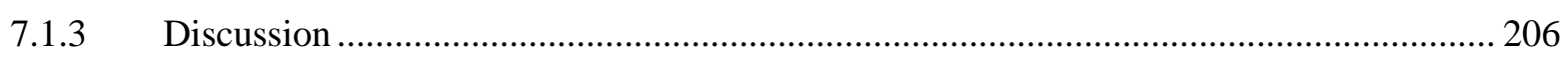

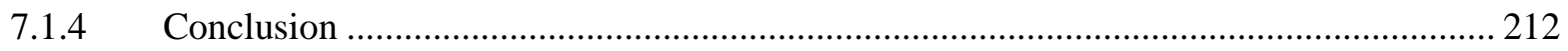

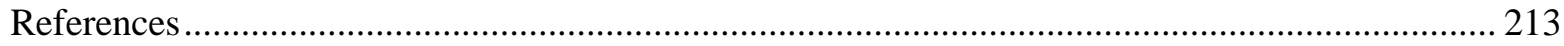

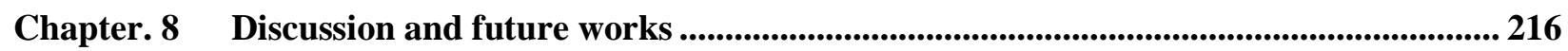

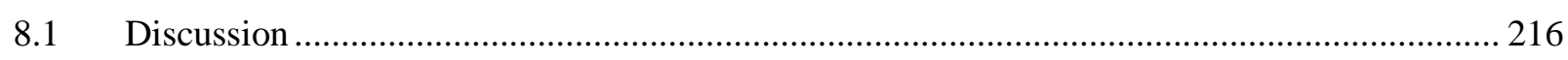

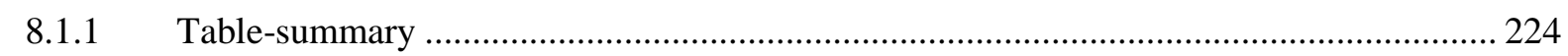

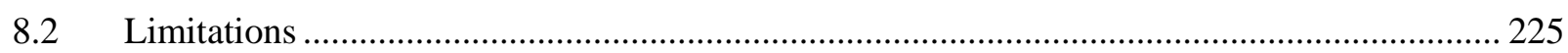

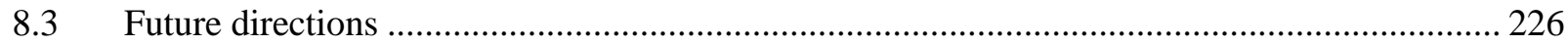

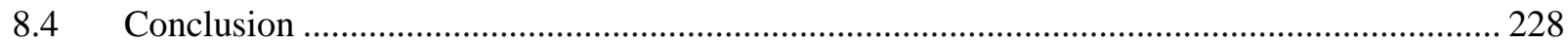




\section{List of figures}

Figure 1-1: The three deterministic substrate factors influencing cell behaviors 3

Figure 1-2: (a) The extracellular matrix of cells, cell cytoskeleton through $B$-integrins. (b) Diverse extracellular signals which induce specific cell behaviors................................................................ 4

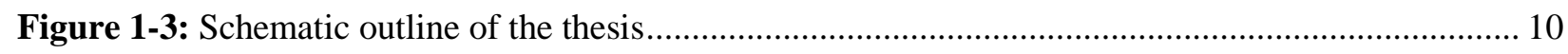

Figure 2-1: Cell differentiation and migration during embryonic germ layer formation and origin of adult human tissues originates from the distinct germ layers.

Figure 2-2: Composition of mammalian cell microenvironments consisting of variety of extracellular molecules, cells and complex assemblies .................................................................. 18

Figure 2-3: Transmembrane integrins, Cell cytoskeletal fibers and matrix proteins forming the focal adhesion points and schematic representation of integrin signaling complex ................................ 19

Figure 2-4: Mesengenic process of hMSC differentiation to somatic cell lineages................................ 22

Figure 2-5: The mesenchymal stem cell niche ........................................................................... 23

Figure 2-6: Mammalian neurogenisis and early cortical plate formation from neural stem cell proliferation, differentiation and radial migration from sub-ventricular zone of brain............................ 24

Figure 2-7: Neuronal niches, signals, integrins and active genes during neurogenesis .......................... 26

Figure 2-8: Comparison of in vitro and in vivo study bio-systems...................................................... 28

Figure 2-9: Influence of surface chemistry on the formation of focal adhesion complexes .................... 31

Figure 2-10: Protein adsorption modes and binding mechanisms on various functionalities and substrate hydrophobicity and its relevance in focal adhesion cluster formation...................................... 32

Figure 2-11: hMSC shape and lineage control on ECM protein micro-contact printed shapes and pattern based modulation of stem cell morphology and cell fates....................................................... 34

Figure 2-12: Micropatterning of glass or plastic culture substrates ........................................................ 35

Figure 2-13: Alignment of neuronal cell processes and cytoskeleton on laminin, recombinant IgG protein (rHIgM12) and poly-1-lysine adhesive micro-contact printed lines........................................... 37

Figure 2-14: Organosilane molecules used to form functionalized SAMs for enhanced reactivity ........ 39

Figure 2-15: Sensing of substrate topography by membrane bound proteins and proteins involved in the formation of focal adhesion points at integrin binding sites.

Figure 2-16: Schematic representation of bone marrow niche and effect of material stiffness and topographical variations in directing hMSC differentiation 42

Figure 2-17: Impact of micro-pillar assemblies, topographical micropatterns and their positioning on the growth and morphology on nascent neurons.

Figure 2-18: Schematic illustration of components and systems available to date that can be integrated into a fully automated lab-on-a-chip for stem cell analysis

Figure 2-19: Schematic illustration of multiple tissues on chip integrated system.

Figure 2-20: Molecular structure of PDMS and the process of replica molding and transfer of micro-features from pholithography derived silicon master wafer

Figure 3-1: Schematic representation of positive and negative photolithography routes....................... 69

Figure 3-2: SEM micrograph of the hole-pillar and ridged pillar-hole-groove topographical

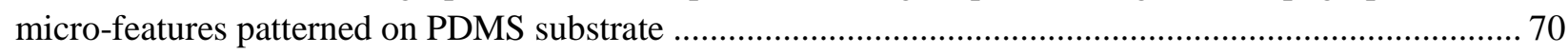

Figure 3-3: Confocal profile micrograph of circular micro-trap feature patterned on PDMS .................. 72

Figure 3-4: Measurement of water contact angle of the static/sessile droplet ....................................... 74 
Figure 3-5: Morphological analysis of hMSCs by cytoskeleton/nucleus staining and morphological analysis by SEM imaging

Figure 3-6: Dissection steps involved in the isolation of cortical hemisphere from the E18/P1 rat brain.

Figure 3-7: Quantitative assessement of neurite angle (ImageJ) and neurite tracing using Neurolucida 360 and Neurolucida explorer

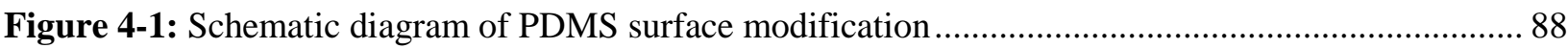

Figure 4-2: XPS scan of $C_{-}$1s and N_1s regions on unmodified and modified PDMS surfaces ............. 90

Figure 4-3: Measurement of average contact angle on TCP, and different PDMS surfaces ................... 91

Figure 4-4: Measurement of protein (Col1 and fib) retention on the modified/unmodified PDMS surfaces after one day of incubation.

Figure 4-5: Adherent cell density after 90 min of initial HMSC seeding on modified/unmodified PDMS surfaces with either collagen type 1 or fibronectin protein coating.....

Figure 4-6: Mean and median cell spread area on modified or unmodified PDMS surface displayed in Box and Whiskers plots.....

Figure 4-7: Cell-spread area of HMSCs on modified/unmodified PDMS surfaces ...

Figure 4-8: PrestoBlue assay for the proliferation of HMSCs cultured on PDMSmodified/unmodified surfaces with collagen type 1 or fibronectin coating

Figure 4-9: Schematic illustration of PDMS surface modification and subsequent cell culture.

Figure 4-10: Average contact angles measured on unmodified and chemically modified PDMS surfaces, with and without Coll coating.

Figure 4-11: Substratum topography (A) and RMS roughness (B) of unmodified and chemically modified PDMS surfaces, with and without Col1 coating.

Figure 4-12: Amount of surface bound protein (Col1) measured on the unmodified and chemically modified PDMS surfaces on day 1, day 7 and day 14, respectively....

Figure 4-13: Investigation on osteogenic differentiation of HMSCs and HMSC sheet formation on unmodified/chemically modified PDMS substrates (TCP control)

Figure 4-14: (A) Assessment of biomineralization on unmodified/chemically modified substrates by Alizarin red staining (B) Expression of osteogenic gene biomarkers by RT-PCR.

Figure 4-15: (A) Collagen formation on unmodified/chemically modified PDMS surfaces as revealed by Picro-Sirius red staining under polarizing microscope (TCP control) (B) Col1 gene expression of HMSC sheet after 2 weeks of culture on unmodified/chemically modified PDMS surfaces.

Figure 4-16: $\beta$-Catenin gene biomarker expression on unmodified/ chemically modified PDMS substrates with Col1 immobilization.

Figure 4-17: Schematic representation of surface silanization and collagen immobilization followed by hMSC culture on flat substrates and PDMS micro-channels.....

Figure 4-18: (a) Water contact angle, (b) Measurement of protein retention, (c) Root mean square (RMS) nano-scale surface roughness (AFM) and (d) XPS analysis of silanized/unmodified substrates....

Figure 4-19: Cell morphology, coverage and initial adhesion on the modified/unmodified substrates

Figure 4-20: (a) F-actin profiles and (b) Mean and median cell spread area on unmodified/native surfaces displayed in Box and Whiskers plots. 
Figure 4-21: Proliferation of hMSCs on silanized/unmodified substrates measured on several intervals between Day 3 to Day 15 by the presto-blue assay.............................................................. 134

Figure 4-22: Morphology of adherent cell sheets within silanized PDMS micro-channels. .................. 135

Figure 4-23: Assessment of calcium bio-mineralization and osteogenic gene expression following

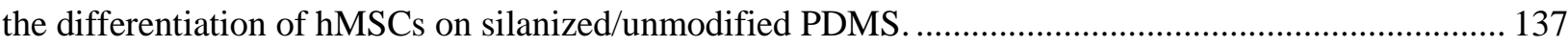

Figure 5-1: Schematic representation of PDMS surface modification and cortical neuron culture......... 146 Figure 5-2: Spectral analysis of (A) N 1s (nitrogen) and (B) C 1s (carbon) elemental composition by region XPS scans on native PDMS and APTES treated PDMS substrates................................... 147

Figure 5-3: Measurements of the average contact angle various PDMS surfaces................................ 148

Figure 5-4: Amount of proteins retained on the APTES modified and protein adsorbed PDMS

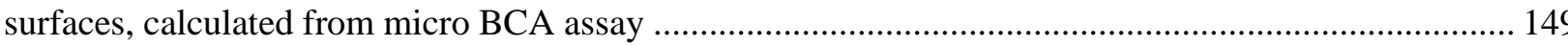

Figure 5-5: (A) Day 21 image set of cerebral cortex cell culture on APTES+protein modified PDMS surfaces. (B) Morphological analysis of attached neuronal cells by mean neurite length measurements and neurite branch count.

Figure 5-6: Immunostaining of neurons (MAP2 antibody) and glial cells (GFAP antibody) (21 DIV) on (A) fibronectin (B) collagen (C) laminin and (D) poly-l-lysine protein surfaces with/without APTES crosslinking. (E-G) SEM micrographs of APTES+poly-l-lysine modified PDMS substrates.

Figure 5-7: Cell culture and immunostaining of neurons (green/MAP2) and glial cells (red/GFAP) cultured for 21 DIV on poly-1-lysine immobilized surfaces on compartmental PDMS chip.

Figure 6-1: (a) Fabrication of PDMS based hole/pillar/groove architectures and subsequent surface modification by APTES+GA+protein crosslinking chemistry.

Figure 6-2: Cytoskeletal (F-actin) and nuclear (DAPI) staining of adhered hMSC cell layers (Day 14) on microfabricated and Flat PDMS substrates

Figure 6-3: SEM micrographs of hMSC adhesion and cell sheet formation preferences on H-P-G and H-P microfabricated substrates 173

Figure 6-4: Comparison of cell adhesion on H-P-G, H-P and flat PDMS substrates. 174

Figure 6-5: Prestoblue analysis of the hMSC proliferation on micropatterend (HPG and HP) and flat PDMS substrates measured at 3, 7 and 14 day intervals.

Figure 6-6: (a) Assessment of calcium bio-mineralization after osteogenic differentiation of hMSCs (b) Relative expression of osteogenic genes (RT-PCR) on micropatterned/Flat PDMS substrates.

Figure 6-7: Illustration of mechanisms of hMSC adhesion and cytoskeletal filament organization in response to individual topographic elements

Figure 7-1: Silicon wafer with micro-trap geometrical array of topographical feature fabrication and schematic representation of cortical neuron culture on micro-fabricated substrates.

Figure 7-2: Neuronal cell entrapment and neurite guidance through the micro-traps in various geometries and quantification of possible orientations and deflections wrt various reference axes

Figure 7-3: Neuronal morphology within the angled/curved micro-traps and neurite extensions through the micro-traps analysed with bright-field, B-III-tubulin immunostaining, DAPI imaging

Figure 7-4: F-actin distribution and orientation profiles along angled, cornered, flat, curved profiles

Figure 7-5: (a-f) Comparison of neurite lengths/neurite numbers, exit tendency and distribution along angled/curved cues. (g) Percentage wise comparison of neurite exit and branching tendency 
Figure 7-6: Micro-gap sensing and neurite guidance along the external profiles of the micro-trap arrays and the possible role of glial cell orientation/guidance.

Figure 7-7: Neuronal networks formed on angled and curved geometric arrays.

Figure 7-8: Graphical analysis of neurite directionality, length \& density in neuronal network

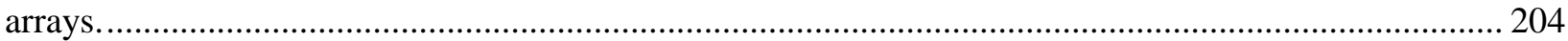

Figure 7-9: Proposed mechanism of F-actin distribution and filipodial growth in response to various angular cues.

\section{List of tables}

Table 1: Review of traditional and recent in vitro strategies to study cell-substrate interactionsx

Table 2: Mesenchymal stem cell responses to surface chemical cues

Table 3: Neuronal cell responses to surface chemical cues

Table 4: Mesenchymal stem cell responses to biophysical cues. 222

Table 5: Neuronal cell responses to biophysical cues 222 


\section{Abbreviations}

\begin{tabular}{ll} 
AFM & Atomic force microscopy \\
ALP & Alkaline phosphatase \\
APTES & (3-Aminopropyl) triethoxysilane \\
APTMS & (3-Aminopropyl) trimethoxysilane \\
ASCs & Adult stem cells \\
C1/Col1 & Collagen type-1 \\
cDNA & Complementary DNA \\
CVD & Chemical vapor deposition \\
CNS & Central nervous system \\
DAPI & 4',6-diamidino-2-phenylindole \\
ECM & Extra-cellular matrix \\
FN & Fibronectin \\
FAK & Focal adhesion Kinase \\
GA & Glutaraldehyde \\
hMSCs & Human mesenchymal stem cells \\
HSCs & Hematopoietic stem cells \\
LBL & Layer-by-layer \\
MPTMS & (3-Mercaptopropyl) trimethoxysilane \\
NSCs & Neural stem cells \\
PCR & Polymerase chain reaction \\
PDMS & Poly (di-methylsiloxane) \\
RNA & Ribonucleic acid \\
SAMs & Self-assembled monolayers \\
SEM & Scanning electron microscopy \\
SVZ & Sub-ventricular zone \\
TCP & Tissue culture plate \\
XPS & X-ray photoelectron spectroscopy \\
\hline
\end{tabular}




\begin{abstract}
Mammalian cell behaviors are highly regulated by the complex cell microenvironments. In vitro models with precise manipulation of cell-substrate interactions can provide realistic insights into the chemical and topographical signals which critically affect cell functions. Poly(dimethylsiloxane) (PDMS) based systems are increasingly used in in vitro cell studies due to several advantages such as low cost, mechanical viability and convenience of rapid prototyping of cellular/subcellular environments. However, the high surface hydrophobicity and low surface reactivity of PDMS surfaces impose serious practical limitations in long-term studies of complex yet therapeutically significant behaviors such as the mesenchymal stem cell (hMSCs) and neuronal cell systems. To address these critical problems, a silanization based chemical modification of PDMS surfaces was employed against the traditional approaches, for covalent immobilization of extracellular matrix (ECM) proteins. The resulting modification promoted stable adhesion, highly spread morphology and viability of hMSCs with stronger cell sheet formation which positively affected cell differentiation. The strategy could be successfully applied to facilitate long-term culture and osteogenic differentiation within PDMS micro-chip. Moreover, the inclusion of hybrid physical micro-features further enhanced hMSC adhesion and differentiation. Eventually, the ECM proteins relevant in nascent neuron development were immobilized by silanization route, which resulted in a healthy neurite density and morphology. Additionally, diverse physical signals in neuronal cell vicinity was studied on micro-fabricated geometrical arrays of varying angularity/curvature which critically affected neurite growth, branching and directional commitment. Taken together, a tunable model was developed to study the independent and synergistic influences of both chemical and physical cues on both cell types. The simple, reproducible and adaptable system devised in this work, could be readily employed in strategic design of cell instructive bio-materials and interfaces to promote tissue regeneration as well as to understand the intricacies and abnormalities associated with cell development.
\end{abstract}




\section{Chapter. 1 Introduction}

\subsection{Background}

To be able to understand and manipulate biological environments has been a focus of diverse areas ranging from environmental to biomedical applications. The genetic code or information related to overall cellular functions are contained in the DNA, transcribed into RNA and finally expressed as a translated protein with defined functions. This phenomenon was termed as the 'central dogma of molecular biology' in 1956 by Francis Crick who had discovered the structure of DNA [1]. The main encoding parts of DNA popularly known as 'genes' are the key intrinsic factors which determine normal cell functions by expressing as proteins as per the sequences in the gene. Although, the innate gene actions autonomously control cell behaviors, a multitude of the signals that control cell behaviors are extrinsic and are triggered from the dynamic surrounding micro-environments. The cell niche or micro-environment also known as extracellular matrix (ECM) is an ensemble of surrounding cells, blood vessels, signaling molecules, growth factors and proteins which are essential for the normal functioning and survival of cells. These niches are also associated with biophysical complexity due to varied topography, stresses and mechanical cues which mediate cell survival, migration and differentiation [2]. Since cell-ECM relationship is critical for normal biological expression of cells and tissues, manipulation of biochemical and biophysical nature of ECM has a regulatory effect on cell behaviors [3, 4]. In the field of tissue engineering, such manipulations in stem cell and nascent mammalian cell environments have been carefully investigated and harnessed for achieving diverse therapeutic end results [3, 5-8]. Since the discovery of mesenchymal stem cell (hMSC) potency in the 1970s, hMSCs have been highly harnessed to differentiate into various tissue lineages such as cardiac (heart), osteogenic (bone), neuronal (brain) and myogenic (muscle) lineages [9-12]. Since then, extensive 
research on biomaterials has focused on creating artificial organs or functional tissue units using stem cells to repair injured/damaged tissues [3,8]. Most research in this realm has dealt with creating bio-interfaces which mimic native tissue like ECM features to be potentially used as an implant to promote tissue regeneration or as a versatile in vitro study system to further understand the practical aspects of hMSC based tissue regeneration. Similarly, since cell-ECM relations are closely associated with cell development, the highly complex tissue environments such as the brain tissue have been studied to uncover the effects of microenvironmental manipulations on nascent neuronal cells and their eventual development. With the advent of micro/nanofabrication technology, there has been a rising interest to develop simple, adaptable and reliable in vitro models which simulate diverse cell-material interactions. Ultimately such investigations are aimed to promote a systematic understanding about the anomalies and underlying environmental factors which affect cell morphology, viability, differentiation or development [13-17].

\subsection{Cell-substrate interactions}

Cells are sensitive to the signals from the surrounding external stimuli. Changes in the composition of these factors in the immediate cell environments during growth/disease/maintenance processes have a direct regulatory effect on the cell behavior [2, 4]. Besides the biochemical factors, the cells sense various physical signals prevalent in the ECM such as the micro/nano-topography, roughness, geometry and stiffness [15, 18]. In this regard, both differentiating cells such as hMSCs and nascent un-differentiated cells such as the neuronal cells have incredibly complex biochemical and biophysical micro-architectures within the bone marrow and the nerve/brain tissues respectively. 


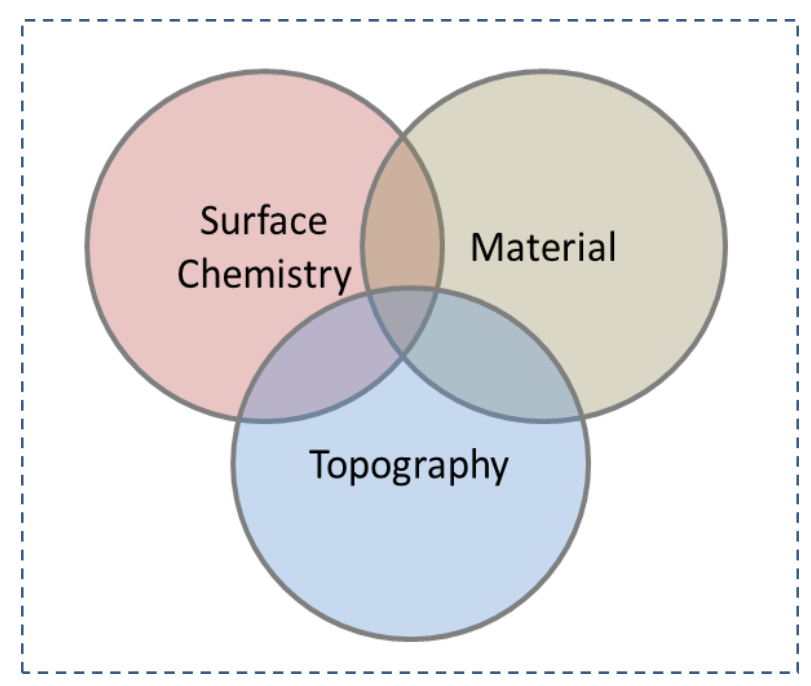

Figure 1-1: The three deterministic substrate factors influencing cell behaviors

Along with the normal physiological conditions required for cell survival (E.g., pH, temperature, soluble factors and serum proteins), there are three key external determining factors which can influence the overall cell behaviors: the surface chemistry, topography and the innate properties of the material (Figure 1-1). These factors co-exist and any change in the degree or nature of one factor will influence the other two factors. This opens diverse avenues to manipulate the material properties as well as chemical/physical interaction of contacting substrate to guide cell behaviors to desired cell fates. The advent of micro and nanotechnologies greatly enabled precise manipulations of cell environments which form the basis of tissue-engineering and biomaterial based regenerative therapies [3]. Subsequently these techniques greatly enabled in vitro investigation of complex cellular behaviors and tuning of these behaviors by micro-environmental manipulation to achieve either therapeutic end-result or to gain deeper knowledge of cell development. Recognizing the micro-environmental cues that affect cellular functions provides a comprehensive understanding of cells and enables to design of suitable approaches to engineer artificial tissues, strategies and in vitro platforms to study cell development. 


\subsection{The ECM and in vitro control of cell functions}

The bone marrow [2] and the neuronal cell niches $[16,19]$ are highly organized and complex. Specifically, cell niches are comprised of a variety of factors such as cytokines, chemokines, growth factors and a wide array of biochemical, biomechanical and bioelectrical signals. A major class of molecules that closely interacts and signals with cells is the ECM which is comprised of proteins such as collagen, laminin, fibronectin and polysaccharides such as hyaluronic acid as shown in

Figure 1-2a [20]. The cells interact with ECM proteins through their membrane proteins (E.g., integrins) and depending upon the nature of underlying ECM proteins or the microtopography, such adhesion triggers a cascade of intracellular signaling which leads to changes in cell fates (E.g., growth, morphology, migration and differentiation).
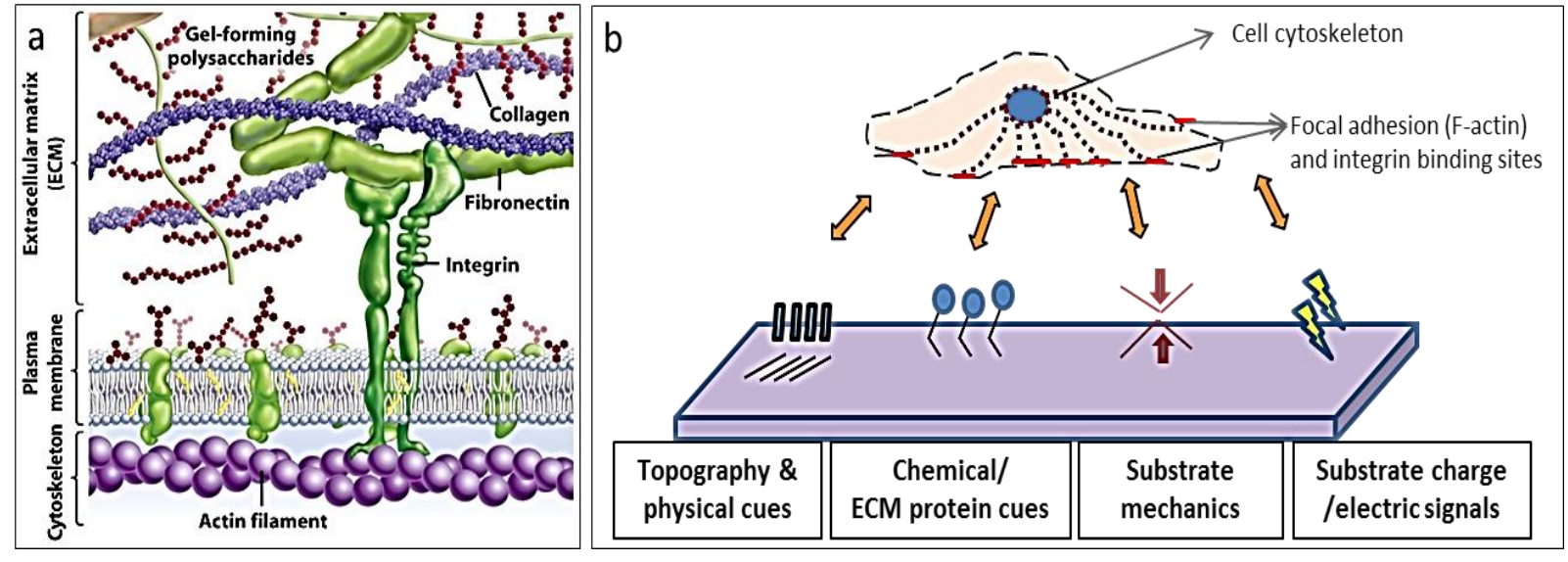

Figure 1-2: (a) The extracellular matrix of cells and their connection with cell cytoskeleton through B-integrins. Adopted from reference [1] (b) In vitro platforms to mimic ECM architectures and induce specific cell behaviors by physical/chemical/mechanical/electrical signals.

Due to high inherent complexity of mammalian cell microenvironments, there is a growing need to create reliable in vitro platforms to decipher the individual/combined influences of extrinsic factors (physical/chemical/mechanical/electrical signals) on cell behaviors and to precisely study cell responses ( 
Figure 1-2 b). To emulate suitable in vivo environments in a laboratory setup, it requires a thorough understanding of natural bio-chemical and physical environments of the cells.

Since cell-surface interactions through chemical/physical external stimuli affect the nature of cell adhesion and key behaviors, precise models which trigger specific cell responses such as differentiation, migration and proliferation have been developed. However, the development of simple, stable, reliable and well-defined in vitro models to investigate cell-material interactions is a core requirement for cell development and tissue engineering studies. A variety of materials and material interface strategies are developed to mimic and test tissue functionalities and to develop repair strategies with close consideration of native tissue architectures and conditions [3, 21-24]. In recent years, the congenial material properties of poly(dimethylsiloxane) (PDMS) has been extensively exploited to design in vitro platforms because of the ability of rapid prototyping of cell and biological environments [25]. Moreover, the tuneability of the material features attracted significant interest in generating micropatterned /microfluidic platforms often decorated with biomolecules to emulate cell niches.

\subsection{Problems and Research gaps}

Considering both in vivo complexity and growing demand for biomaterial based tissue regenerative therapies, there is a need for accurate in vitro determination of cell responses to various surface chemical and biophysical cues. Conventional biomimetic systems such as plain 2D cultures with poorly defined chemical and topographical substrate features are limited in terms of robustness and diversity of environmental signals. Further such systems are prone to inaccuracies in assessing certain key cell behaviors. In recent years, cell studies based on PDMS systems are increasingly employed for various tunable advantages and the ability to rapid prototyping of diverse features in various size and arrangements [25, 26]. However, the high hydrophobicity of PDMS surfaces poses critical limitations in studying complex and challenging cells microenvironments as the stem cells and neuronal cells, due to low surface biocompatibility which result in poor adhesion of cells and biomolecules $[25,27,28]$. Moreover, the traditionally used physical adsorption of ECM proteins on PDMS surfaces are 
characterized with poor biocompatibility due to the eventual loss of bioactivity and dislodgement of adsorbed cell or protein layers within 1week of culture. This enforces the use of expensive materials and fabrication procedures often compromising on the overall quality and applicability of PDMS platforms to long term and highly critical cell behavioral investigations. Mesenchymal stem cell differentiation to specific tissue lineage is dependent on wide variety of complex biochemical and topographical signals which critically affect their focal adhesion, survival and differentiation [29]. In this regard, conventional culture systems and low reactivity of PDMS pose problems in assessment of dynamic cell behaviors which affect these processes. Similarly, the complex and critical morpho-regulatory parameters affecting neurite growth, morphology and networking during nascent neuronal development are difficult to investigate in such systems. In the light of these issues, there is a need to create stable, simple and reproducible platform to study ECM influences on behaviors of mammalian cells of therapeutic and developmental significance. 


\subsection{Motivation}

Conventional in vitro platforms offer limited insight into overall cell interactions with biochemical and biophysical environments, due to various material and interface limitations. Although PDMS based supports are increasingly used in cell studies due to several advantages, there are some practical limitations associated with surface reactivity towards cells and the overall interaction of surface topography and chemistry in regulating certain important cell fates (highlighted in the previous section). Moreover, cell niches such as the mesenchymal stem cells and neuronal cells share common characteristics in terms of complexity, composition and difficulties associated in deciphering cell functions in lab scale. These limitations provide a motivating basis to utilize the advantages of PDMS and address the issues concerning surface reactivity/biophysical manipulation and develop simple, tunable, reproducible and versatile strategies which can enable studies of diverse cell functions with therapeutic significance. These investigations hold a potential to offer methods to flexibly functionalize ECM proteins or ligands of interest and stabilize functional cells/ cell groups/ cell sheet in various controlled architectures (E.g., microfluidics/micro-patterned supports). Eventually these platforms could be used as a tool to study influences of various stimulatory signals which affect cell morphology, survival and differentiation into specific lineages which are important in the perspective of developmental and tissue engineering studies. 


\subsection{Objective}

The overall aim of this work is to design simple yet efficient in vitro platform to study the influence of surface chemistry and topography on key cell behaviors. The specific objectives of this study are

1. Fabrication of stable, reproducible and tunable platform to study microenvironmental influences on cell behaviors

- Surface chemical modification by silanization

- Micro-fabrication of topographical features or microfluidic channels

$\circ$ Optimization and improvement of PDMS surface characteristics and biocompatibility

- Characterization of altered substrate properties

2. Investigation of cell behaviors on altered PDMS hydrophobicity/chemical composition induced by surface chemical modification

- Characterization of hMSC cell adhesion, morphology and proliferation

- Characterization of neuronal cell adhesion and survival

$\circ$ Analysis of neurite growth and morphology

3. Investigation of the effects of topography and substrate geometry on cell behaviors

- Characterization of hMSC anchorage, morphology, proliferation and potency

$\circ$ Assessment of neuronal cell preferential alignments, neurite growth and branching

4. Study of the combined effects of chemical and topographical cues on cell fates

- Identify the specific and synergistic factors which direct hMSC fate and neuronal development 


\subsection{Novelty and significance}

The role of ECM proteins and the micro-topography on mammalian cells are discussed in several past studies. However, the diverse ECM influences especially in the realm of directing critical cell processes such as hMSC adhesion and differentiation and the factors involved in early neuronal development in complex environments such as the brain are sparsely understood. Stable in vitro systems with a capability to easily manipulate the biochemical and biophysical surroundings of the cells and long-term influence of cell behavioural responses have not been sufficiently explored. To our knowledge, this is the first study to apply a silane based PDMS surface bio-functionalization strategy to stabilize mammalian cell adhesion and to eventually investigate key cell fates for $\geq 21$ days in vitro. Further, the effect of molecular structure and functionality of the self-assembled monolayers (SAMs) of silanes and the nature/conformation of immobilized ECM proteins on cell fates were carefully investigated with a successful implementation of the strategy on the PDMS micro-channels. Moreover, past studies have majorly focused on several isolated chemical/topographical influences. However, the combined influence of various topographical elements with surface chemistry in complex and therapeutically relevant cell systems such as hMSC and neurons are not known. To address these issues, a novel micro-fabricated substrate was developed which could encompass diverse topographical signals varying in size, scale and geometry. This design approach was used to enable detailed investigation of several contributing factors affecting cell behaviours and physiology. In case of hMSC cells, in vitro evaluation of cell adhesion and several critical processes which affect hMSC cell sheet stability and potency was evaluated. Hence the novel platform could be used to study diverse factors affecting these critical behaviours which have relevance in cell sheet engineering, micro-environmental regulation of cell differentiation and strategic design of in biomaterials for tissue regeneration. For neuronal studies, the silane based surface chemical modification was employed to create a novel and easily reproducible long-term investigation platform to assess neuronal $\mu$ cell morphology and viability in response to covalently immobilized ECM protein cues. Eventually, a novel micro- 
patterned model was designed to investigate the specific morphological regulation and cytoskeletal reorganization of isolated neurons and neuronal networks in response to diverse array of geometrical biophysical cues. This platform could be applied in detailed neuro-morphological screening, investigation of neuro-developmental anomalies and neuronal guidance based repair strategies. Overall, easily fabricable and tunable biointerfaces were developed to facilitate the study of diverse cell-micro-environment influences which control critical cell behaviors in complex tissue niches.

\subsection{Thesis layout}

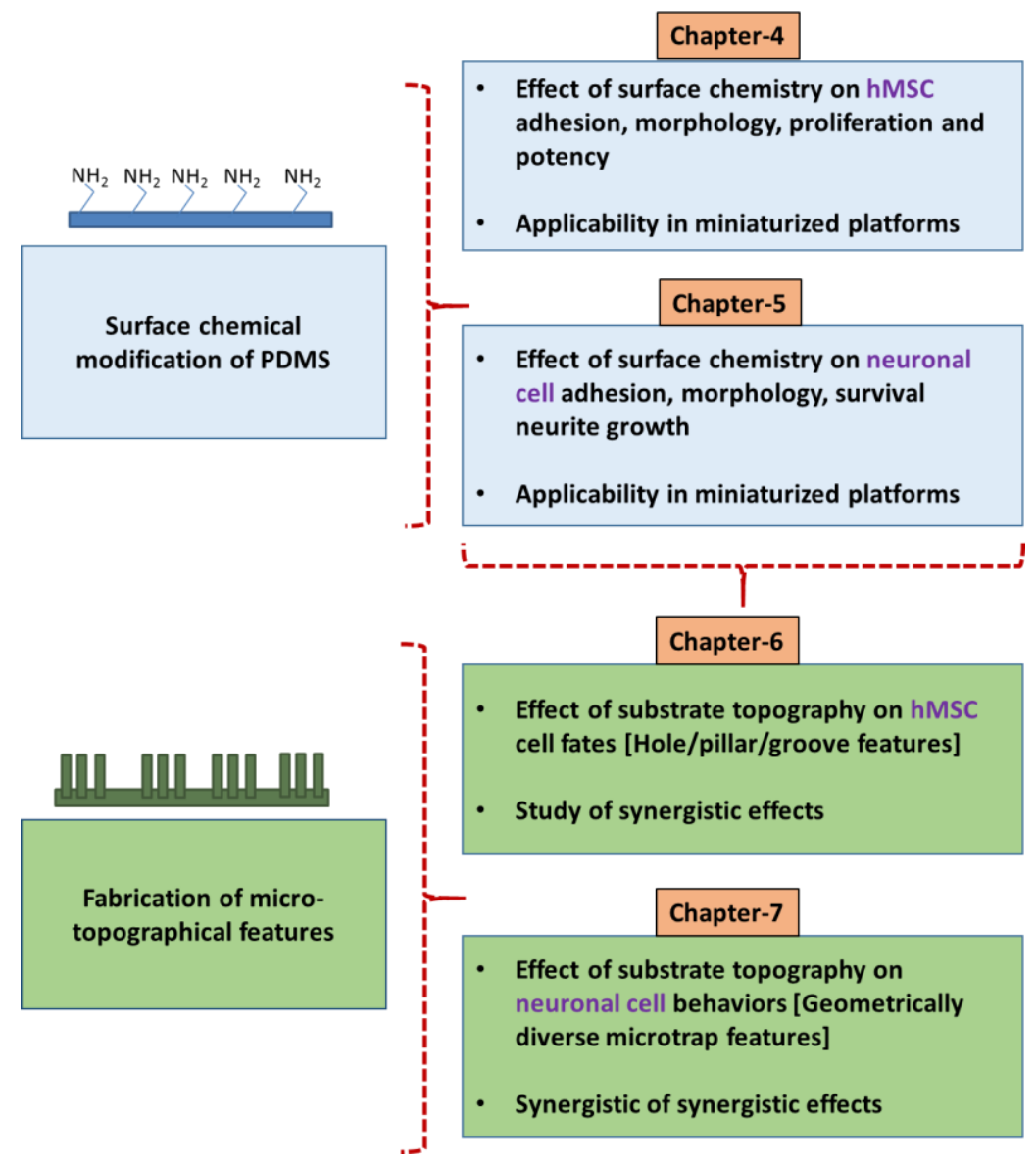

Figure 1-3: Schematic outline of the thesis

Figure 1-3 presents a schematic overview of the thesis. In the first part of the thesis (Chapter 4 and 5), surface chemical modification was employed to optimally minimize PDMS surface hydrophobicity and stabilize ECM protein adhesion. The resultant surface is characterized for 
both surface chemical and roughness changes as well as biocompatibility and fates of hMSCs is presented in Chapter 4 as three sub-sections as follows. The effect of chemical modification on hMSC morphology, spreading, adhesion and viability is presented in section 4.1. The influence on hMSC osteogenic cell fate and cell sheet formation on silanized supports is explored in section 4.2. The effect of silane type, molecular structure/functionality on cell fates and its implementation in a PDMS microfluidic model is presented in section 4.3.

The effect of surface chemical modification on neuronal growth, morphology and development is presented in Chapter 5. In the second part of the thesis (Chapter 6 and 7), microfabrication of topographic features, relevant characterization of surface features is performed and the eventual influence on cell behaviors is studied. Firstly, the effect of hole, pillar and groove based 3D like topographic substrate on hMSC morphology, adhesion, cell sheet formation, and osteogenic differentiation is studied (Chapter 6). In this chapter the combinatorial influences from surface chemical modification, along with topographical regulation is also discussed. The subsequent chapter (Chapter 7) deals with geometrically mediated topographic steering of neurite behaviors and network formation in cortical neurons. In this chapter, the effect of various topographic elements varying in angularity and curvature on morphological regulation and cytoskeletal variation is studied on both individual neurons and neuronal network subsets. Chapter 8 presents a discussion on the key inferences drawn from Chapters 4-7 and presents overall outlook of the major objectives addressed in the thesis. This chapter also proposes directions for future works based on several perspectives and outcomes drawn from the projects. 


\section{References}

[1] Watson, J.D. and F.H.C. Crick, Molecular Structure of Nucleic Acids - a Structure for Deoxyribose Nucleic Acid. Nature, 1953. 171(4356): p. 737-738.

[2] Jones, D.L. and A.J. Wagers, No place like home: anatomy and function of the stem cell niche. Nature Reviews Molecular Cell Biology, 2008. 9(1): p. 11-21.

[3] Ali Khademhosseini, J.B., Mehmet Toner, Shuichi Takayama, Micro and Nano engineering of the cell microenvironment: Technologies and Applications. 2008.

[4] Adams, J.C. and F.M. Watt, Regulation of Development and Differentiation by the Extracellular-Matrix. Development, 1993. 117(4): p. 1183-1198.

[5] Curran, J.M., R. Chen, and J.A. Hunt, The guidance of human mesenchymal stem cell differentiation in vitro by controlled modifications to the cell substrate. Biomaterials, 2006. 27(27): p. 4783-4793.

[6] Dalby, M.J., et al., The control of human mesenchymal cell differentiation using nanoscale symmetry and disorder. Nature Materials, 2007. 6(12): p. 997-1003.

[7] Ladran, I., et al., Neural stem and progenitor cells in health and disease. Wiley Interdisciplinary Reviews-Systems Biology and Medicine, 2013. 5(6): p. 701-715.

[8] Langer, R. and J. Vacanti, Tissue engineering. Science, 1993. 260(5110): p. 920-926.

[9] Bunnell, B.A., et al., Adipose-derived stem cells: Isolation, expansion and differentiation. Methods, 2008. 45(2): p. 115-120.

[10] Ogliari, K.S., et al., Stem cells in dermatology. Anais Brasileiros De Dermatologia, 2014. 89(2): p. 286-291.

[11] Thomson, J.A., et al., Embryonic stem cell lines derived from human blastocysts. Science, 1998. 282(5391): p. 1145-1147.

[12] Vellasamy, S., et al., Isolation and characterisation of mesenchymal stem cells derived from human placenta tissue. World Journal of Stem Cells, 2012. 4(6): p. 5361.

[13] Alvarez-Buylla, A. and J.M. Garcia-Verdugo, Neurogenesis in adult subventricular zone. Journal of Neuroscience, 2002. 22(3): p. 629-634.

[14] Barros, C.S., S.J. Franco, and U. Muller, Extracellular Matrix: Functions in the Nervous System. Cold Spring Harbor Perspectives in Biology, 2011. 3(1).

[15] Hoffman-Kim, D., J.A. Mitchel, and R.V. Bellamkonda, Topography, Cell Response, and Nerve Regeneration. Annual Review of Biomedical Engineering, Vol 12, 2010. 12: p. 203-231.

[16] Kazanis, I. and C. Ffrench-Constant, Extracellular Matrix and the Neural Stem Cell Niche. Developmental Neurobiology, 2011. 71(11): p. 1006-1017.

[17] Magdaleno, S.M. and T. Curran, Brain development: Integrins and the Reelin pathway. Current Biology, 2001. 11(24): p. R1032-R1035.

[18] Kulangara, K. and K.W. Leong, Substrate topography shapes cell function. Soft Matter, 2009. 5(21): p. 4072-4076.

[19] Low, V.F., et al., Neurogenesis and Progenitor Cell Distribution in the Subgranular Zone and Subventricular Zone of the Adult Sheep Brain. Neuroscience, 2013. 244: p. 173-187.

[20] Frantz, C., K.M. Stewart, and V.M. Weaver, The extracellular matrix at a glance. Journal of Cell Science, 2010. 123(24): p. 4195-4200.

[21] Dowling, D.P., et al., Effect of Surface Wettability and Topography on the Adhesion of Osteosarcoma Cells on Plasma-modified Polystyrene. Journal of Biomaterials Applications, 2011. 26(3): p. 327-347.

[22] Han, Y., et al., Formation process and structure of polyproline self-assembled monolayer on gold surface. Abstracts of Papers of the American Chemical Society, 2011. 241.

[23] Lee, J.H., et al., Cell Behavior on Polymer Surfaces with Different FunctionalGroups. Biomaterials, 1994. 15(9): p. 705-711. 
[24] Kinney, M.A. and T.C. McDevitt, Emerging strategies for spatiotemporal control of stem cell fate and morphogenesis. Trends in Biotechnology, 2013. 31(2): p. 78-84.

[25] Lee, J.N., et al., Compatibility of mammalian cells on surfaces of poly(dimethylsiloxane). Langmuir, 2004. 20(26): p. 11684-11691.

[26] Duffy, D.C., et al., Rapid prototyping of microfluidic systems in poly(dimethylsiloxane). Analytical Chemistry, 1998. 70(23): p. 4974-4984.

[27] Fuard, D., et al., Optimization of poly-di-methyl-siloxane (PDMS) substrates for studying cellular adhesion and motility. Microelectronic Engineering, 2008. 85(5-6): p. 1289-1293.

[28] McDonald, J.C., et al., Fabrication of microfluidic systems in poly(dimethylsiloxane). Electrophoresis, 2000. 21(1): p. 27-40.

[29] DiMarino, A.M., A.I. Caplan, and T.L. Bonfield, Mesenchymal Stem Cells in Tissue Repair. Frontiers in Immunology, 2013. 4: p. 201. 


\section{Chapter. 2 Review of the literature}

\subsection{Mammalian cell differentiation and development}

To design cell type dependent strategies for tissue repair and regeneration, it is essential to know the characteristics of both the cells and their immediate micro-environments. The mammalian cell commences at an early embryonic stage until the hierarchical formation of specific tissues and organs in various stages. During these stages, the cells are exposed to various changes in their niche which influence their division, migration and organization into specialized tissue. Various soluble factors and extracellular matrix proteins are also exchanged into the cell and from the cell to the external environments resulting in the creation of specialized environments called 'niches' with defined biochemical and mechanical characteristics.

In the context of mammalian developmental biology, the mammalian cells could be broadly classified as undifferentiated cells (E.g. stem cells) and differentiated cells (E.g somatic cells) cells. The term 'differentiation' refers to the process in which the cells transforms from one state (undifferentiated, stem cells) to another (differentiated daughter cells). Throughout the mammalian development, differentiation occurs several times right from the zygote stage to the formation of complex tissues and organs. The factors affecting differentiation may however depend on the cell type and the cascade of gene regulatory networks associated with the cells [1]. The mesenchymal stem cells (hMSCs) and hematopoietic stem cells (HSCs) originate from the mesoderm. The HSCs generate the blood cells. Nevertheless, all the germ layers which generate the adult tissues originate from the embryonic stem cells through a series of cellular re-organizations which occur post fertilization (Figure 2-1)[2]. 


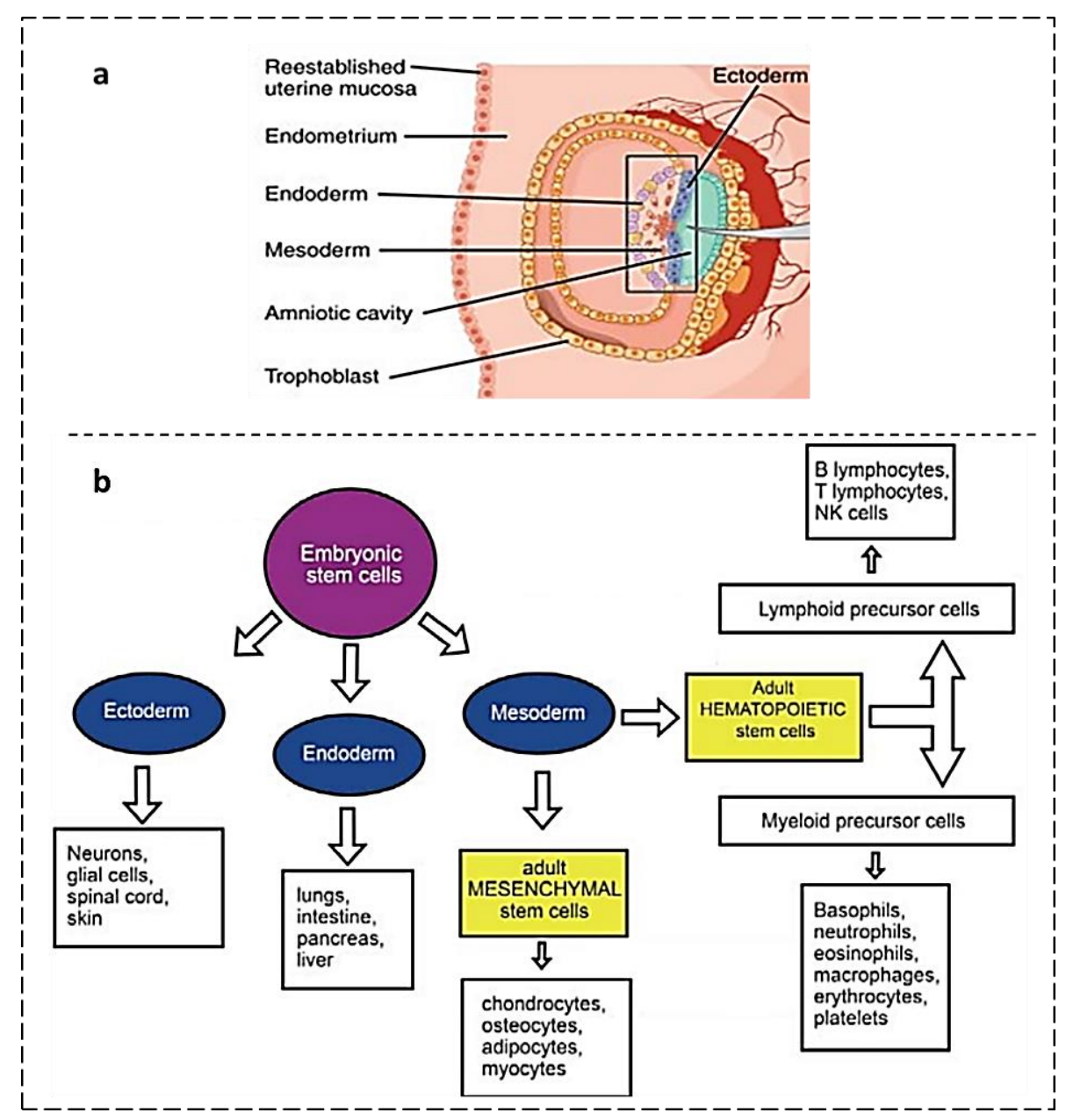

Figure 2-1: (a) Structure of the mammalian embryo. Adapted from reference [2] (b) Adult human tissues originates from the embryonic stem cells. During growth, differentiation into adult tissues occurs from all three germ layers. The adult stem cells originate from the mesoderm layer. Adapted from reference[3].

hMSCs which are prevalent in the bone marrow can differentiate to any tissue type based on the signaling molecules which are also known as growth factors. Further, the response of cells to growth factors/surrounding molecules may vary depending on the cell or tissue type [3]. Apart from the hMSCs and HSCs, the body also houses specialized adult stem cells (ASCs) such as skin stem cells and neuronal stem cells which can only differentiate towards the respective cells. These stem cells are involved in the continuous replacement, repair and renewal of damaged tissues during the normal growth and survival of the organism. 


\subsection{Cell microenvironments and Cell-ECM relationships}

The hypothesis of cell 'niche' was first coined by Schofield (1978) while referring to the extracellular environments of stem cells[4]. The niche was also regarded by Jones et.al, [5] as a 'defined anatomical compartment' which signals the stem cells and control their proliferation, determine the fate of their lineage and protect cells from dying. Majorly, the composition of ECM is dominated by three types of bio-macromolecules: Proteins, proteoglycans (carbohydrates conjugated with protein fibers) and non-proteoglycan polysaccharides [5-7]. The main proteins which make the fibrous ECM are collagen, fibronectin, laminins and elastins $[4,5]$. Among proteins, collagen in the form of collagen-I and collagen-IV subtypes form the bulk of the ECM protein. These proteins could be present in various configurations, types and sub-types each performing specific function in the overall behavior of ECM. For instance, collagens provide structural support to cells while fibronectin affects the re-organization of cell cytoskeleton and assist in cell morpho-dynamics and migration[8]. The fibronectin backbone is made of multiple sequences of amino acid tandem units such as RGD, RGDS, LDV, and REDV which play an active role in cell adhesion $[9,10]$. Similarly, laminins provide structural support and assist in cell adhesion by interacting through PDSGR, YIGSR, and IKVAV sequences[11]. Heparan sulfate, chondritin sulfate and keratan sulfate are the major proteoglycans which maintain the water buffering of the tissue environments. Hyaluronic acids are the major non-proteoglycan molecules, which regulate tissue development, tumor formation and inflammation [6-8, 12-14]. The dynamic behavior of ECM is required to maintain certain biomechanical properties such as tensile and compressive strength and elasticity of organs for which the buffering capacity and water retention nature of ECM is critical. ECM acts as a connective plug between the cells and the materials and directs the morphological organization and function of cells. This is accomplished by binding to signaling molecules/growth factors (GFs) and communicating with cell membrane-surface receptors to trigger signal transduction and eventual gene transcription within cell nucleus[8]. Variations in these interactions could trigger degradative pathways resulting in the loss of 
critical cell and tissue functions which could also result in organ failure. Hence it is essential to identify the independent and synergistic role of diverse microenvironmental factors which affect cell behaviors.

\subsection{Impact of ECM composition and properties on cell behaviors}

The ECM may directly control cell function through receptor-mediated signaling. Alternatively, the ECM may regulate cell proliferation and differentiation by dynamic mobilization of signaling molecules and differentiation factors[15]. Aplin and Juliano (1999)[16] indicated that different ECM macromolecules can selectively trigger specific signal-transduction pathways. They emphasized that the successful proliferation or differentiation of cells, requires an active interaction of ECM components with the cells and vice versa. 


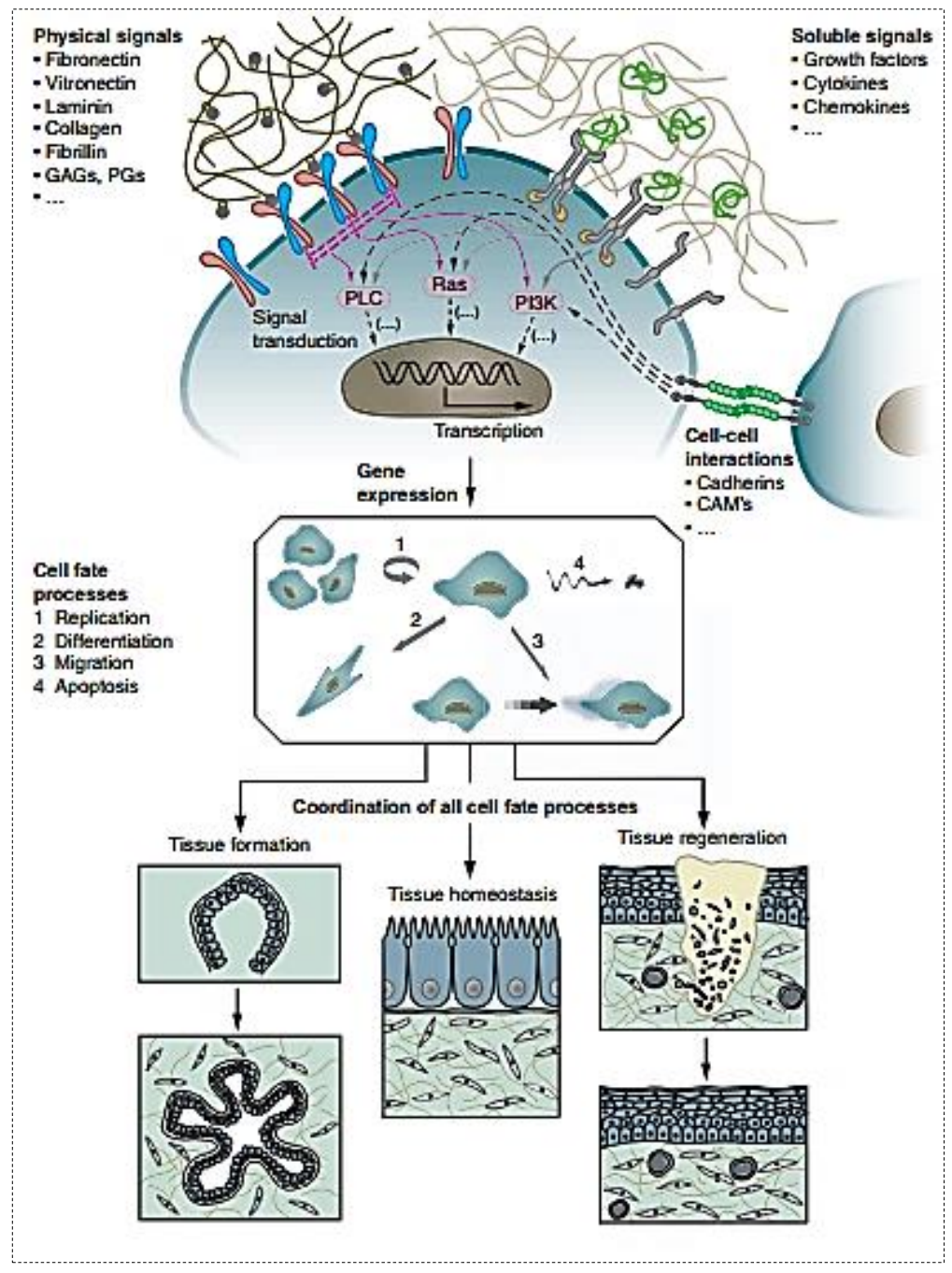

Figure 2-2: Cell microenvironments consisting of variety of extracellular molecules, cells and complex assemblies. Adapted from reference [21]

The active involvement of cell surface receptors ( $\beta-1$ integrins) and signaling molecules was also shown by Howe et.al, 1998[17] . Ortiz et.al, 1995[18] showed that the binding of neutrophils to fibronectin triggered the cells to synthesize tumor necrosis factor (TNF). Nevertheless, activation of specific cellular signals involves specific ECM $\rightarrow$ protein, $\mathrm{ECM} \rightarrow$ growth factor and $\mathrm{ECM} \rightarrow$ surrounding cell interactions as summarized in Figure 2-2. From the early developmental stages, cells such hMSCs, nascent brain cells and the ESCs secrete a variety of molecules into their surroundings $[19,20]$. During eventual development of cells into tissues and tissues into organs, the diverse spatial organization of these molecules produce natural niches in which cells continue to proliferate, migrate and differentiate into 
resultant lineage cells based on the signals from their natural substrates. This encompasses an active involvement of wide array of growth factors and ECM proteins which play an instructive role for various cell activities [21]. Besides ECM composition, the overall biomechanical make-up of the surrounding ECM also involves matrix features such as its microtexture, topography, elasticity and tensile strength which are important in the context of the whole tissues $[8,21]$. The cells can sense the ECM mechanics majorly from the membrane bound $\beta-1$ integrins [17].

\subsection{Regulatory role of Integrin-ECM composition cytoskeleton}

\section{crosstalk}
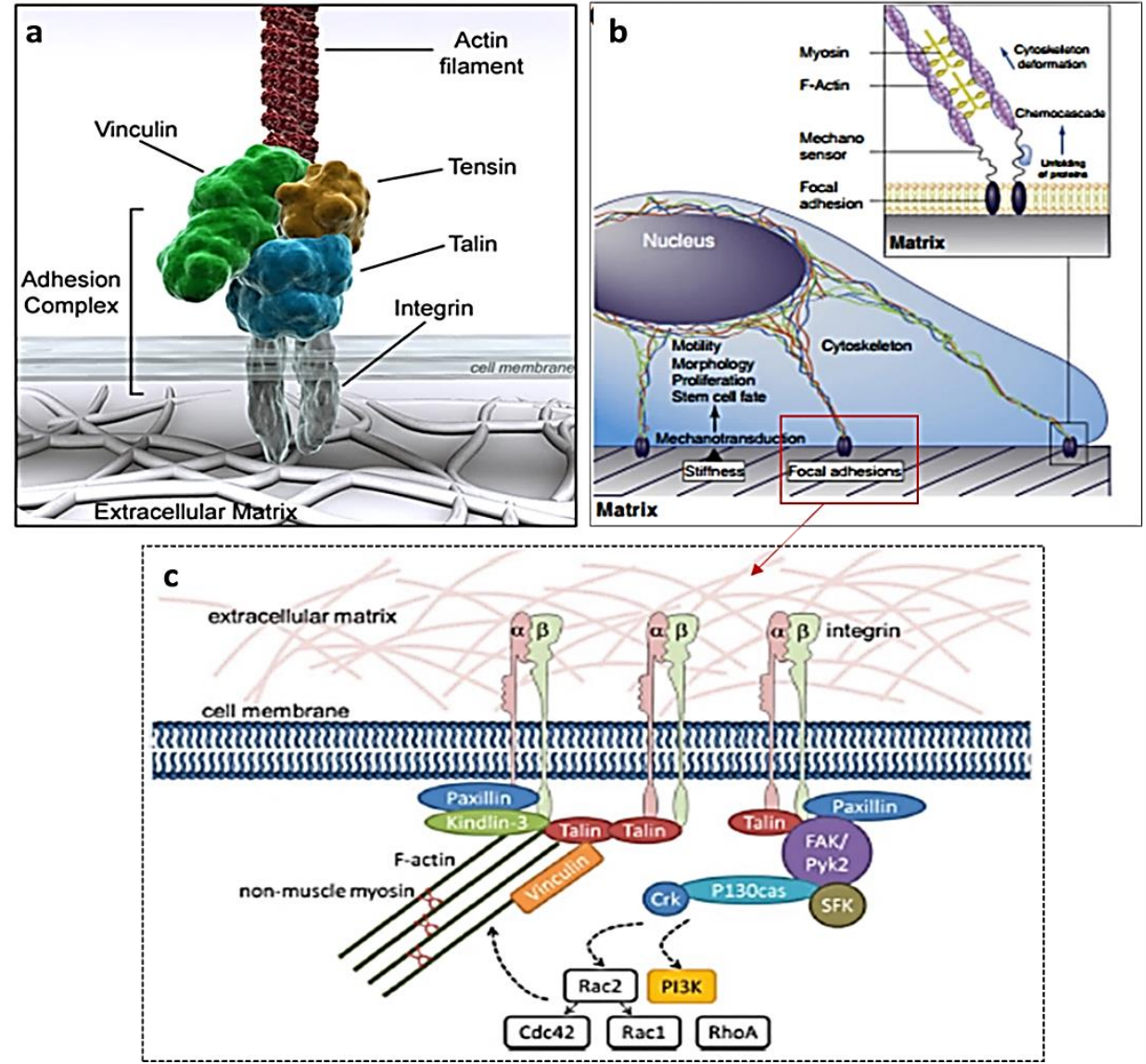

Figure 2-3: (a) Trans-membrane integrin supporting actin cytoskeleton and anchoring the extracellular (fibronectin) protein. Adapted from reference [25] (b) Cell cytoskeletal fibers composed of actin myosin attaching to the matrix through integrins at focal adhesion points and connecting cell nucleus. Adapted from ref. [25]. (c) Schematic representation of integrin signaling complex. Adapted from reference [26] 
The cells interact with the surrounding ECM through the membrane bound transmembrane receptors which control cell anchorage on the supporting matrix within tissues. Along with discoidin and syndecans, one of the major class of ECM proteins that specifically interact both with cell cytoskeleton and underlying ECM proteins are the integrins[22-25]. Integrins are involved in two-way transmission of both signals into the cell and out of the cells and act as an active link connecting cell cytoskeleton to the surrounding ECM (Figure 2-3a). The cell cytoskeleton is in turn connected to the cell nucleus. Hence integrins could be described as effective transmitter of signals from ECM to nucleus. In synchrony with ECM nature, the cells sense the dynamic chemical and mechanical nature of their microenvironment and reflect the traction forces generated at the cytoskeleton back to the ECM through integrins (Figure 2-3b) $[17,21,24]$. The integrin tail is connected to the cytoskeletal fibers (actin filaments) through several adaptor proteins (Figure 2-3 c). These proteins are responsible for integrin clustering during integrin binding with ECM proteins. This assembly resembles a tent wherein the pegs are the integrin clusters or focal adhesions and the ropes of the tent are the actin fibers which connect focal adhesion points with the nucleus. The focal adhesion also has two-way relationship with the cells and the ECM. The cells use focal adhesion to sense local chemical and protein domains and the ECM mechanics (stiffness/hardness/topography etc.). Reciprocally, the focal adhesions also exert traction forces on the underlying substrates[17]. Different classes of integrins have different levels of interactions with ECM and associated actions [17, 24]. Further, the cell cytoskeleton-integrin-ECM assembly (Figure 2-3) is a critical factor in cell survival and overall expression. Past studies have shown that alterations in integrin activity or focal adhesions could occur due to ECM alterations which has been linked to cell death and disease progression in both differentiating and un-differentiated cells [17, 24, 26-28]. In hMSCs, the changes in ECM-cell cytoskeleton interactions due to ECM perturbations may alter the differentiation pathways[29]. Similarly, in undifferentiating nascent cells, these changes are also known to affect key developmental pathways such as the cortex development, neurogenesis and cell migration [30, 31]. 


\subsection{Engineering cells and cell environments for regenerative therapies}

One of the major ways in which cells can be powered for the treatment of diseases is the injection of live cells into the body of the affected patient. For instance, the T-cells which have immunogenic potential can fight the cancer cells through cell-mediated immunity [32, 33]. For this purpose, the self-renewing and differentiation ability of the stem cells have also been utilized to treat specific diseases such as cancer[34] and growth disorders[35]. Advances in the genetic engineering were used to reprogram the natural ability of cancer cells to proliferate and express within the living organisms. However, over the years, an alternative approach of transplanting living cells/bone marrow directly into the organisms by has gained significant interest especially in aiding the recovery of damaged tissues and organs. To overcome the barrier of host body rejection of foreign material and to overcome the bio-incompatibility of the inserted materials, it required to study natural environments of the cells. Since then, cell therapeutics have been extensively focusing on devising means to study the regenerative potential of stem cells and adult somatic cells and to use them for accelerated tissue and organ repair strategies[36]. Although three types of stem cells: embryonic stem cell (ESCs), mesenchymal stem cells (hMSCs) and adult stem cells (ASCs) were considered for therapies, the use of ESCs has been subjected to controversies due to high immunogenicity and aberrant lineages[37]. Hence the low immunogenic and self-renewal ability of hMSCs and ASCs have been greatly studied. Especially, the unique characteristics of the hMSCs from adult bone marrow have been used for several critical bone, cartilage and myocardial tissue therapies. Similarly, the use of the ASCs such as neural stem cells[38] and neuronal progenitor cells[39] have been employed to address the problem of permanent tissue damages inflicted during injury or neurodegenerative diseases such as the Alzheimer's and Parkinson's diseases. In the following section, we discuss the role of hMSC based engineering and the study of nascent neurons in solving critical problems in tissue recovery and nerve/brain development. 


\section{6 hMSCs in tissue engineering and role of stem cell niches}

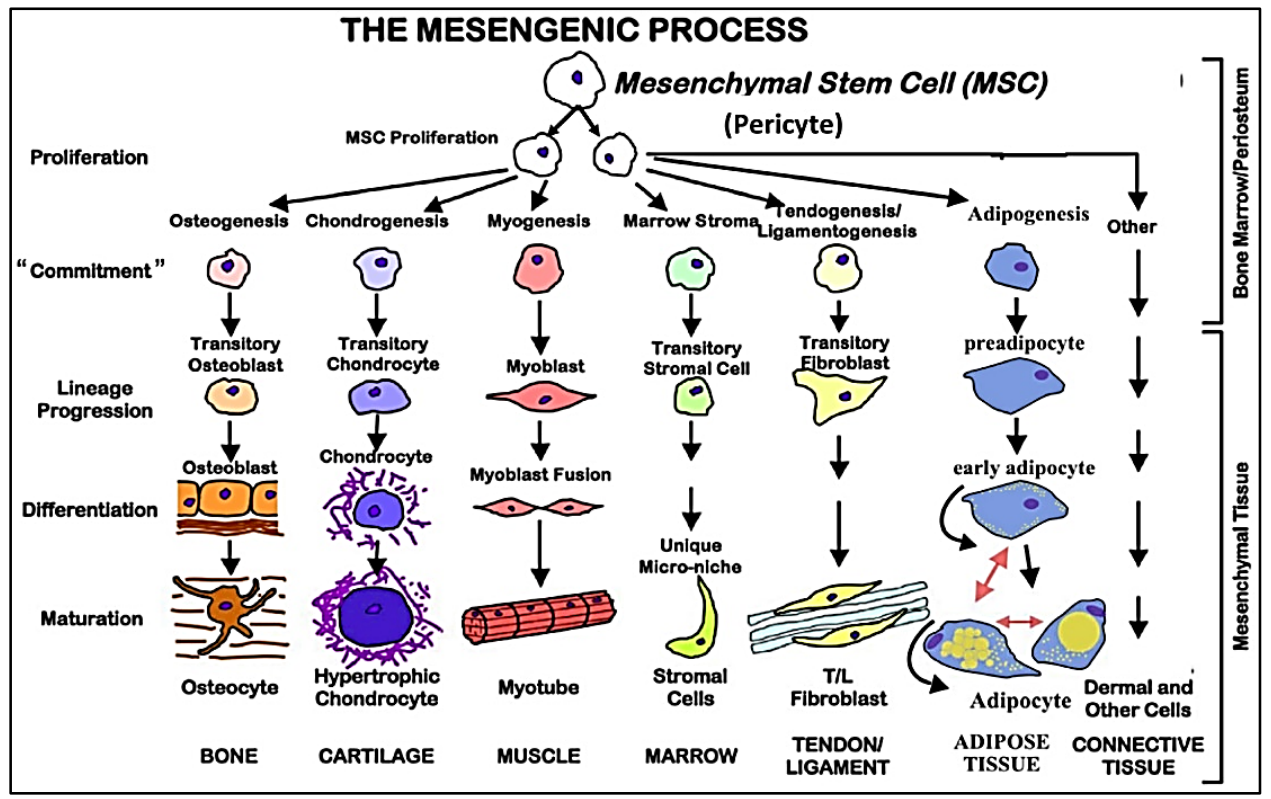

Figure 2-4: The mesengenic process of mesenchymal stem cell proliferation and differentiation into diverse somatic cell lineages. Adapted from reference [45]

The self-renewable ability and multipotency of hMSCs have been widely exploited in tissue engineering. hMSCs possess long term viability and proliferate faster which is a highly advantageous feature. The mesengenic process of hMSCs shows that they can differentiate into several tissue lineages (Figure 2-4). Further, hMSCs in host body are safe due to low immuno-reactivity while obtained from either autologous or allogenic sources. The hMSCs can also be easily harvested from bone marrow which is one of the widely approved sources. In recent years, stem cells have also been extracted from non-marrow sources such as adipose tissue[40], placenta[41], umbilical cord blood [42, 43] and dental pulp[44]. Past studies have shown that the natural hMSC microenvironments are extremely complex and the constituents of hMSC niches control their morphology, proliferation and differentiation[14, 21, 45]. This observation has aroused significant interest to modulate hMSC functions by manipulating their environments. Design of suitable bio-microenvironments and bio interfaces are employed to promote hMSC differentiation into selective/predetermined lineages. hMSCs response to various chemical factors as well as biophysical factors such as matrix stiffness, hardness, 
roughness and topography has raised significant interest to design suitable biomaterials which can induce specific lineage commitments[14, 21, 45, 46]. Hence to mimic specific behaviors of hMSCs to environmental cues, it is critical to have a realistic picture of hMSC natural environment (niches) in which they naturally thrive.

\subsubsection{Cell-matrix relationships in directing $\mathrm{hMSC}$ cell behaviors}

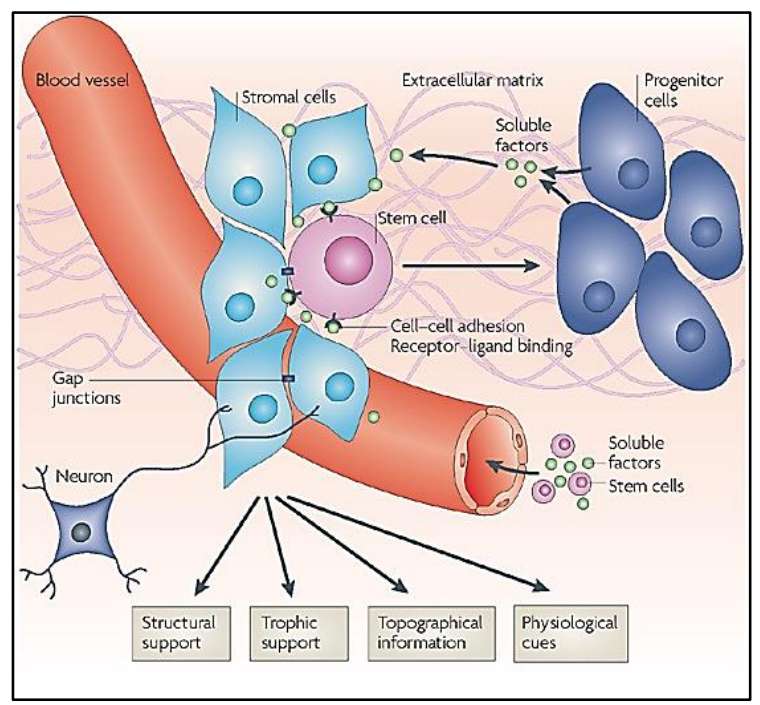

Figure 2-5: The mesenchymal stem cell niche. Adapted from reference [7]

hMSCs are exposed to diverse biochemical conditions which take part in signal transduction, proteins, endothelial tissues, blood vessels as well as neighboring cells[46] (Figure 2-5). Biologically, these elements function in natural synergy to perform overall functions of the tissue. The stem cell niche is composed of a complex mixture of biochemical and biophysical cues. Some of the ECM components are secreted by hMSCs themselves and the surrounding cells during their growth and maturation. Along with fibronectin and collagen, laminin and elastin fibers also interact closely with hMSCSs. Further there is active interaction of several integrin subtypes which activate downstream signaling by focal adhesion kinase (FAK) and phosphoinositide 3-kinase (PI3K)[47, 48]. The $\alpha 6 \beta 1$ integrin is one of the primary receptor which binds to ECM proteins [49]. Despite the biochemical cues, an active substrate factors such as stiffness, topography, ECM proteins, ligands, growth factors, peptides, cell-cell 
communication and mechanical forces in the stem cell niche can significantly affect cell activities[46].

\subsection{Neural development, neurogenesis and neuronal niches}

In mammals, the central nervous system (CNS) which includes the brain and the spinal cord commences its development from the ectoderm stage. During the third week, a neurectoderm is formed at it forms a neural plate. Then, the embryo undergoes a series of cell migration, proliferation and differentiation forming the forebrain, midbrain and the hindbrain[50]. During early embryonic stage and neonatal stage, the neural stem cells in the neural tube undergo several divisions and finally differentiate into the neuron and glial cells. The neurons and glial cells do not divide any further and only few neurons are formed during the growth of the organism from the neural stem cell at the sub ventricular zone (SVZ) of the brain $[51,52]$. Unlike other somatic cells, the neurons are post-mitotic and do not divide any further during the entire lifespan of the organism. This poses a serious challenge since any damage or injury on the CNS may cause permanent disorder/loss of brain functions.

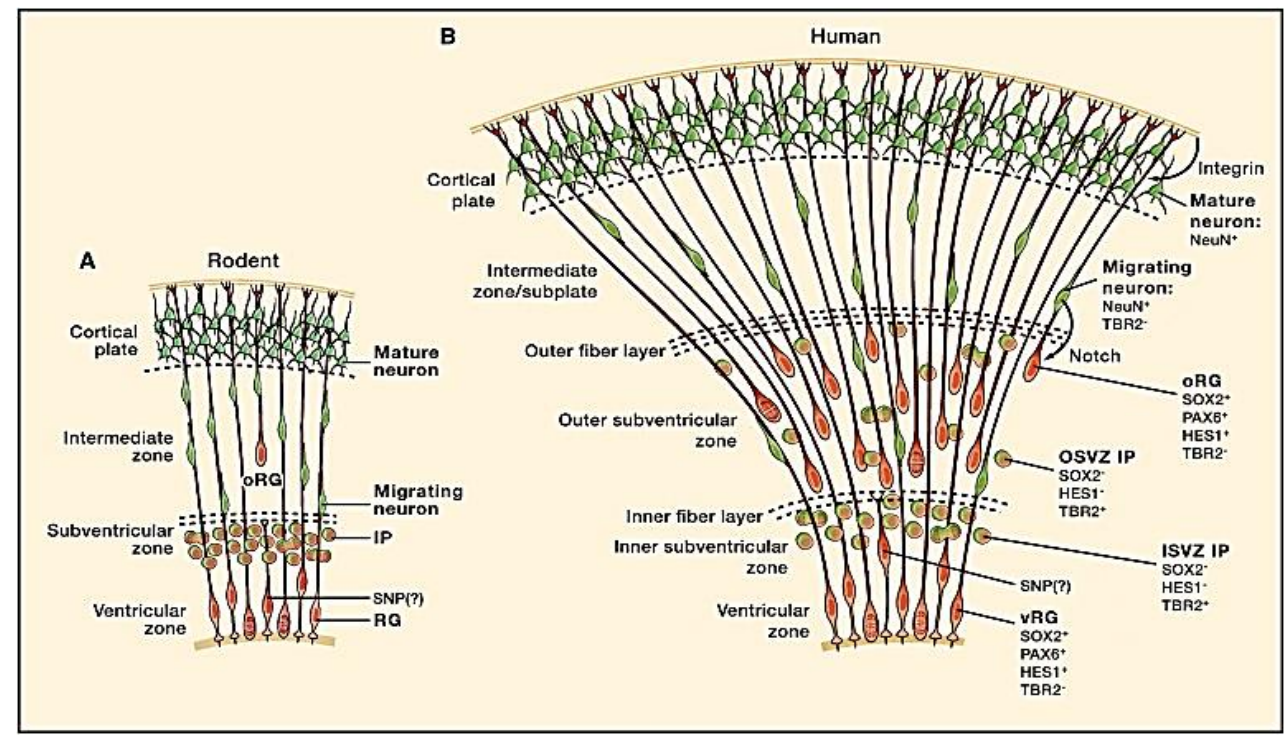

Figure 2-6: Neurogenesis (neuron cell formation) and cortical plate formation from neural stem cell proliferation, differentiation and radial migration from the ventricular zone in (A) rodent brain and (B) human brain. Adapted from reference[53, 54]. 
The process by which neural stem cells and neural progenitor cells form the neurons during brain development[55] is known as neurogenesis. The neural stem cells in the sub-ventricular zone (SVZ) eventually form the neurons, glial cells or oligodendrocytes. In rodents, the neurons which differentiate from the progenitors migrate along radial glial fibers towards the cortical plate and undergo maturation. In humans, neurogenesis occurs similarly but the neuronal migration occurs through two additional layers i.e. the inner and the outer SVZs. Neurons migrate and mature at the cortical plate in the early stages of brain development and form cell-cell contacts called synapses/synaptic junctions (Figure 2-6)[53, 54]. These junctions are vital for the communication between the neurons thorough signal transmitting molecules known as neurotransmitters. The signals are exchanged through an $\mathrm{Na}+/ \mathrm{K}+$ ion channel gated mechanism at the axonal membrane of neurons. During brain development, the neuronal stem cells and neurons undergo several changes through dynamic cell-cell and cell-ECM signaling. Neuronal niches resemble hMSC environments in composition while differing in certain basic biochemical and biophysical elements. Perturbations of the cell-ECM interactions in the neuronal niches can greatly affect the neuronal development and are shown to result in neurodegenerative pathways[56]. Hence to devise efficient neuro-regenerative strategies and biomaterial designs it is vital to understand the critical neuronal cell-ECM interactions 


\subsubsection{Cell-matrix relationships in directing neuronal cell behaviors}

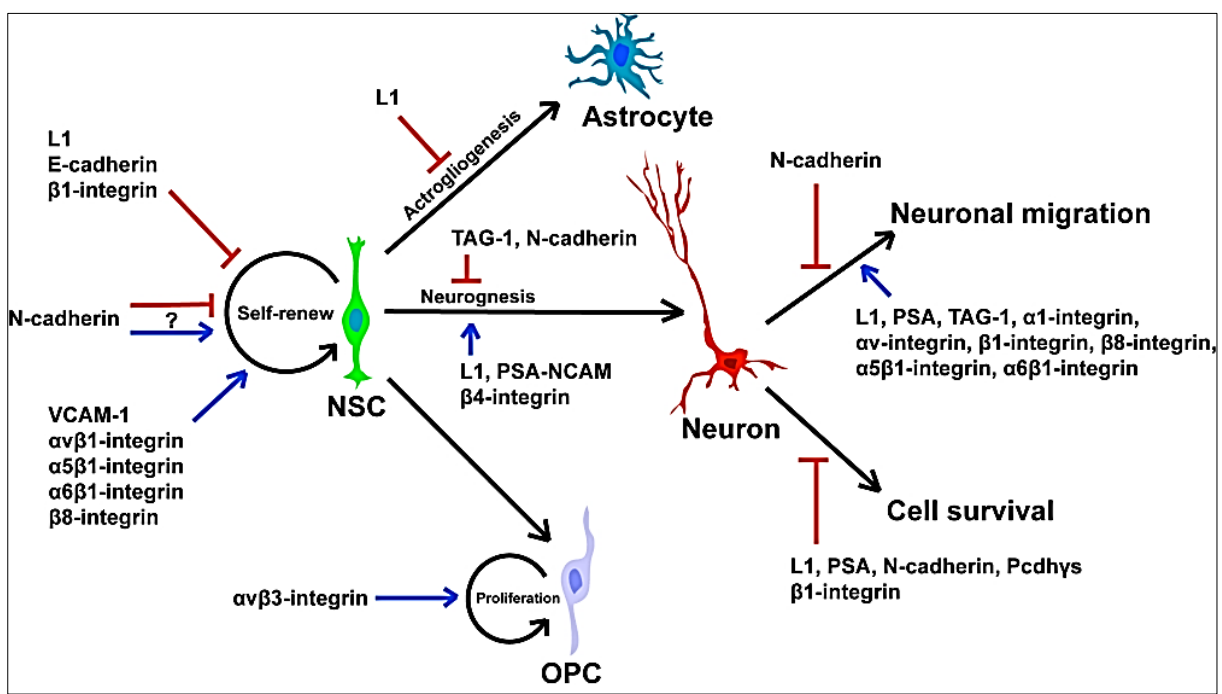

Figure 2-7: Neuronal stem cell and neuronal niches, signals, integrins and active genes during neurogenesis. Adapted from reference [57]

The neuronal niche is vastly dominated by cell-cell interactions and cell-matrix interactions[57]. Figure 2-7 shows the involvement of various regulatory factors and membrane integrins involved in neural stem cell differentiation and nascent neuron migration. The brain tissues are composed of several layers each with varying matrix composition. Apart from the dominant presence of collagen I collagen IV and fibronectin, the most common ECM proteins which dominate the neuronal ECM is laminin[58]. The laminins are family of trimeric proteins made of diverse combinations of five $\alpha$, three $\beta$ and three $\Upsilon$ subunits, with one alpha, one beta and one gamma which make up the trimer of a specific laminin[59]. Staquicini et.al, showed that netrin-4/laminin complex plays an active role in the migration and division of early neuronal progenitors[60]. In addition to astrocytes, neural stem cells are closely associated with glial cells and blood vessels, and are in direct contact with the cerebrospinal fluid (CSF) which contains wide array of regulatory molecules. The ECM also plays a regulatory role in synapse formation. Geissler et al showed that in case of neurons with reduced ECM, the synapses were proved to be less stable, and further number of synapse junctions was significantly reduced [61]. Barros et.al reviewed role of several ECM components and their functions in mediating axon growth and myelination [58]. Several 
soluble factors and ions $\left(\mathrm{Na}^{+}, \mathrm{K}^{+}\right.$and $\left.\mathrm{Ca}^{2+}\right)$ also act in conjunction with proteins and regulate neuron functions $[58,62]$.

\subsection{In vitro mimicking of cell micro environments}

The ability to manipulate cell microenvironment to direct specific cell behaviors is a core focus of biomimetic tissue engineering research. To be able to implant suitable cell laden materials and matrices, it is essential to screen various physiological and biochemical conditions which could be used for rational biomaterial development. Langer. $R$ and Vacanti J.P (1993) have discussed several factors that needs to be considered to recreate versatile cellular environments[63]. For instance, models of liver tissues on chip could be used to study both liver tissue development from induced pluripotent stem cells[64] and screen a large library of drugs which directly affect liver cell behavior and functional recovery[65, 66]. In the following section, we review the rationale for in vitro bio-mimicking and advances in engineering cell environments for tissue engineering and cell development.

\subsubsection{Rationale for in vitro bio-mimicking for regeneration/developmental research}

The nature of mammalian cell micro environments not only differs from species to species, but also differs within the same organism between different tissues and organs. Hence to know the effects of drug on tissue functions, or to know the tissue responses towards various external (mechanical/electrical/chemical) stimuli it is difficult to recreate appropriate animal models each time[67]. Tissue level experimentations involving complex array of experimental or physiological conditions cannot be carried out on animals on a regular basis which would otherwise involve sacrifice of large number of animals and inconsistency in testing various in vivo conditions[67]. 


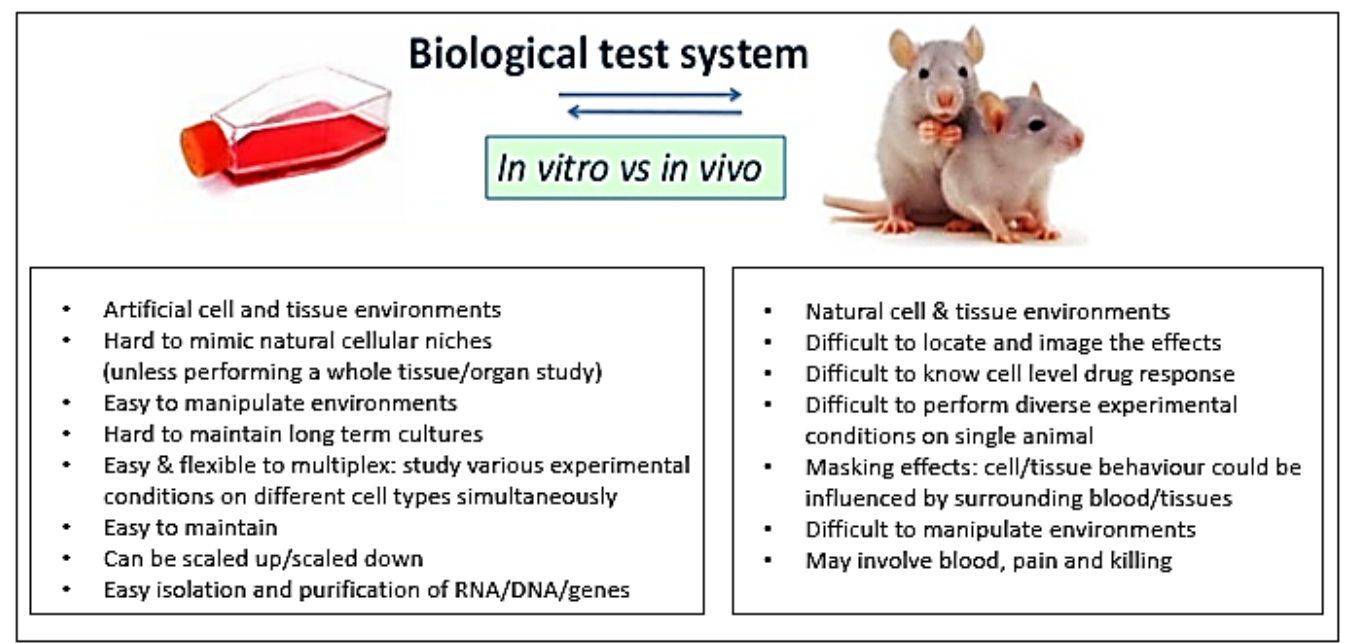

Figure 2-8: Comparison of in vitro and in vivo test systems

A comparison of various advantages of in vitro studies against certain practical limitations in employing animal models is summarized in Figure 2-8. Although animal models represent the most direct and natural scenarios, there are several challenges which could be encountered during detailed investigations. Further the maintenance and treatment of animals involves stringent regulations and animal housing expenses. Over the years, the advances in nanoorganic chemistry, material functional chemistry, protein engineering and micro/nanoscale fabrication were used to create biomimetic models with realistic spatial and temporal resolutions $[67,68]$. These tools can be successfully used to modulate cell environments on a lab scale. Moreover, traditional culture systems such as the coverslip and tissue culture plates have limited cell-cell and cell-substrate interactions and hence do not adequately reflect the natural niche of the cells[69]. Latest advances in micro and nanofabrication, 3D bio-printing and imaging claim to study the hMSC behaviors in carefully controlled niches with finely tuned the matrix properties[69]. Further, due to similarity in experimental requirements, the in vitro experimental platforms could also be tuned to study recovery, development and injury models of challenging tissue environments such the neuronal niche in the brain[70, 71]. In vitro models are aimed to reduce the number of variables and hence simplifies the study. Taken together, these factors have stirred considerable interest to design in-vivo like experimental platforms which could be easily scaled-up or scaled-down based on the experimental end purpose. 


\subsubsection{In vitro manipulation of ECM: Overview and recent trends}

A variety of factors need to be considered while preparing in vitro tools for cell/tissue behavioral investigations. On a broader sense, Khademhosseini et.al, 2008[67] have classified the control of key in vitro factors in three categories.

\section{Control of biochemical and biophysical environments}

This involves spatiotemporal decoration of bio-molecules commonly present in the cell ECM on suitable $2 \mathrm{D}$ or $3 \mathrm{D}$ bio-matrices. These biomolecules directly interact with cell surface receptors and affect specific gene expression pathways. To control these interactions, techniques such as soft lithography and surface modification have been employed. In recent years, cell-chemical or cell-ligand interactions techniques by micro-contact printing of the ligand molecules, growth factors or ECM proteins are done on various $2 \mathrm{D}$ substrates. To create more natural like design, 3D scaffolds are also being developed to control cell interaction in niches with 3D orientations of cells. Such 3D environments are achieved either by suspension cultures of cell spheres, acoustic tweezers, 3D printing, de-cellularized matrices or by micron/nano-scale fibers with layer by layer morphology[69]. The substrate topography is also a major regulatory factor which control cell fates. Fabrication of micro/nano features and topographies has been employed to mimic natural tissue based architectures in heart, and

skeletal tissues. Further the fluidic architectures are also generated to create microvasculature like environments in which the migration and differentiation of several important cell types have been studied[70-73]. Besides, the impact of biophysical environments on cells has also been studied by manipulating the mechanical properties of the substrates such as substrate stiffness and elasticity [74].

\section{Control of soluble cellular micro-environments}

Several soluble factors such as cytokines, chemokines, ions, hormones are closely involved in regulating cell behaviors. In the natural biochemical environments, these factors are present in interstitial spaces of tissues, cell gaps, blood and serum. However, to assess the role of these factors or to create soluble factor based environments, a variety of studies involving methods 
such as microfluidic gradient generation and nano-fluidics has been used. Apart from the conventional gradient systems, recently Caroll et.al, 2014 developed a simplified cell coculture system which could overcome several disadvantages of conventional systems wherein both cell-cell interaction and cell-soluble factor interaction could be studied between an ovarian cancer cell and differentiated macrophage cell lines with high control on dynamics of cell-cell interactions[75]. A similar model was also employed by Chen et-al for high resolution on-chip investigation of T47D (breast cancer) cell interaction with fibroblasts[76]. Although the soluble factors play a key role in mediating cell signals by interacting with cell surface receptors, their expression is a result of overall cell functions which are primarily affected by the substrate chemistry and topography. In the following section, we discuss the influence of in vitro surface chemistry and topography which are the major biochemical and biophysical signals which play a vital role in regulating cell behaviors.

\subsubsection{Role of Surface chemistry and ECM protein in modulating cell behaviors}

The substrate chemistry and underlying ECM protein plays a highly interactive role with mammalian cells and are essential for normal physiological activity. One of the prime cell behavior influenced by the surface chemistry is cell adhesion. An active cross talk of cells with the functional groups, ligands, antigens/antibodies and proteins during cell adhesion influences a cascade of cell behaviors [77, 78]. Cell adhesion and viability on the substrate surfaces depend on substrate characteristics such as wettability/surface energy, reactivity and functionality. To derive a functionally interactive substrate it is desirable to achieve a wellbalanced synergy of all these characteristics. For instance, Dowling et.al, 2011 discussed an active correlation between surface wettability and topography on the adhesion and cell spreading of osteosarcoma cells[79]. They observed optimal cell adhesion in contact angle of $\sim 64^{\circ}$ and with a surface roughness $\left(R_{\mathrm{a}}\right)$ values in the range of $19-2365 \mathrm{~nm}$, while the highly hydrophilic and hydrophobic coatings resulted in lower cell adhesion. In vitro control of cell response to surface composition could be mediated either by direct interaction with the 
functional groups or polymeric/ionic interfaces/ protein or peptide biomolecules that are immobilized by bio-conjugation. Surface chemistry influences cell adhesion and morphology by interacting with the receptors like $\beta$-integrins and the proteins at the cell-substratum contact (E.g. Talin) and affect focal adhesion mechanism of the cells[78].
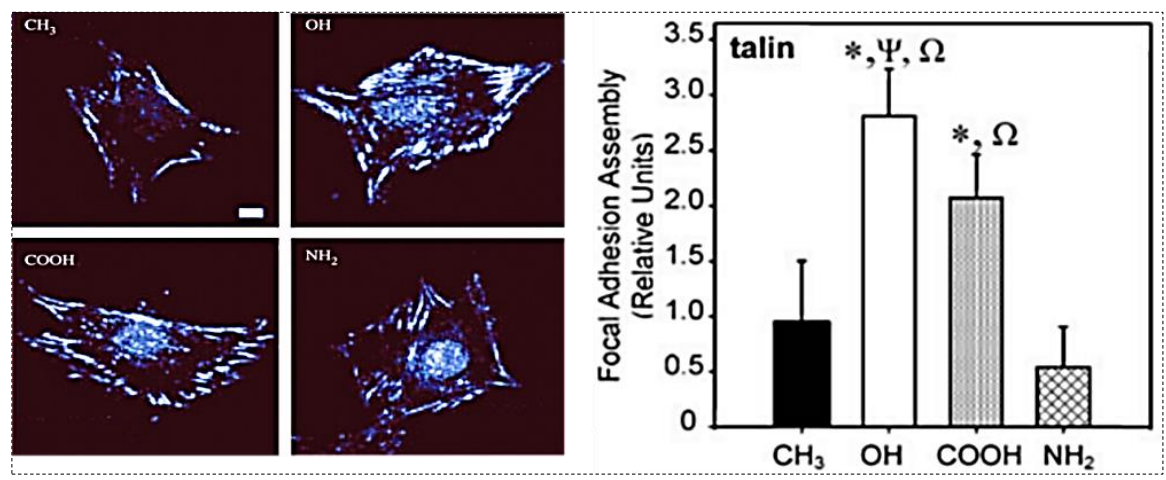

Figure 2-9: Localization of the structural protein talin on SAMs, illustrating how varying the surface functionality influences the formation of focal adhesion complexes. Adapted from reference[78].

Keselowsky et.al, 2004 showed the impact of surface functional groups in mediating the formation of focal adhesion complexes via $\alpha 5 \beta 1$-integrin binding and focal adhesion kinase (FAK) signaling[78] (Figure 2-9). To activate cell material interaction methods such as SAM (self-assembled monolayer) deposition, polyelectrolyte coating by layer-by-layer (LBL) method, chemical vapor deposition (CVD), plasma treatment, patterning with polymers, lipids and PEG have been used on various materials such as silicones, glass, hydrogels, micro/nanofibers and micro/nanoparticles[67, 80]. These methods aim to improve surface reactivity towards cell proteins and enable enhanced cell-substrate interactions. However, most methods have some practical limitations such as low coating stability, low strength of binding, fouling, cross-reactivity, non-specific interactions and cytotoxicity. Hence, there is a need for versatile functional bio-interfaces. 


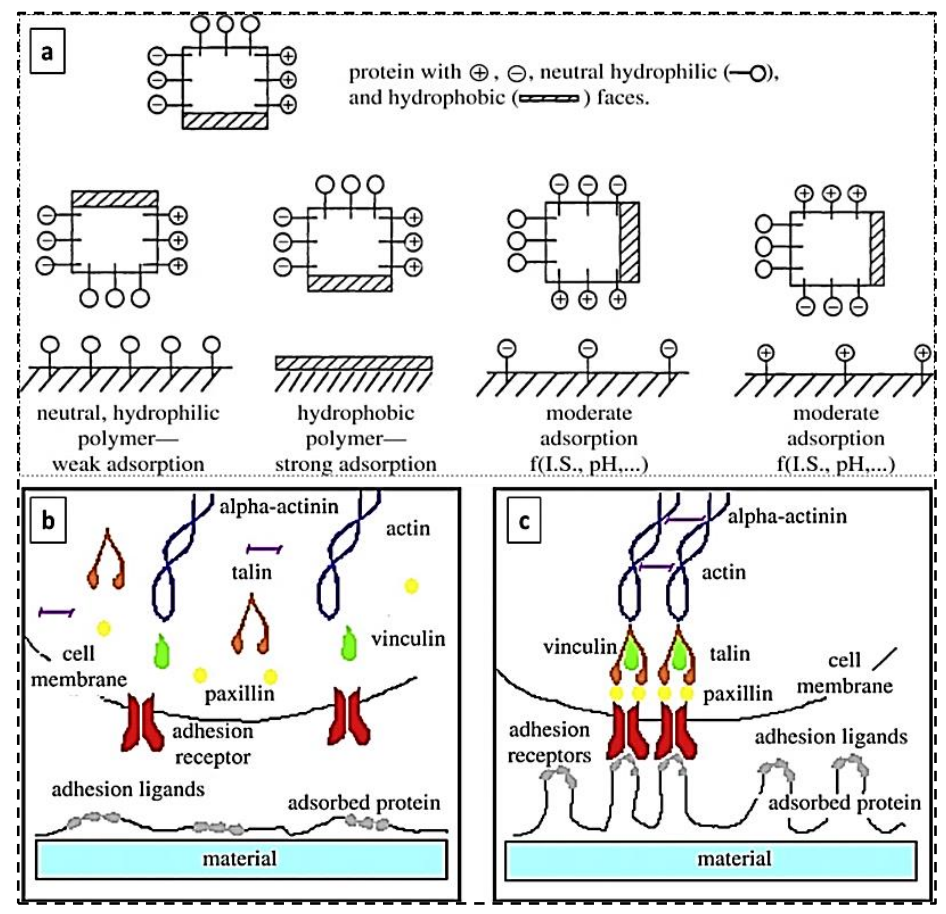

Figure 2-10: (a) Modes of protein adsorption on the different functionalities and hydrophobicity of the substrates. Adapted from reference [88] (b, c) Mechanism of binding of immobilized protein with the cell surface receptors (E.g., integrin) by clustering of receptors and polymerization of cell cytoskeletal proteins at the cell-material interface. Adapted from reference [84]

Protein based surfaces have been developed mostly for biosensor applications and study of antigen-antibody relationships by protein linkage on substrates by both covalent and noncovalent approaches. Due to the ease of approach, most of protein based matrices employed coating of the cell culture plastic wares or glass coverslips with functional peptides, ECM proteins such as fibronectin, collagen, laminin by physical adsorption[81]. Andrade et.al, 1992[82] discussed the protein adsorption on surfaces with varying net charge and polarity (Figure 2-10 a). They showed that strength of adsorption on the matrix depends on the overall charge and polarity of the protein (whether it is mainly hydrophobic, positively charged, negatively charged or neutral hydrophilic). The actual amount of proteins and strength of the adhesion may vary with different complex parameters such as the protein type, charge on the proteins and the hydrophobic/hydrophilic amino acid side chain distribution. Hence, the surface charge, functionality, hydrophobicity and immobilization conditions such as coating 
time, temperature/pH needs to be carefully considered [81]. The overall idea is to identify proteins which are present in the native environments and immobilize them on cell culture substrates to improve cell anchorage by enhanced integrin-cell cytoskeleton and material cross talk [83]. Cell adhesion on the protein matrices occurs by integrin clustering, dynamic rearrangement and polymerization of cytoskeletal protein and formation of focal adhesion points which are the anchorage points of cells with the matrix (Figure 2-10 b, c). Here, the surface hydrophobicity is critical, since the cells prefer moderately hydrophilic surfaces for complete integrin clustering and cell fiber recruitment during FAK signaling pathway as shown by Bacacova et.al 2011[77]. The material surface properties are critical to ensure the overall biocompatibility of the substrate to promote healthy cell adhesion and long-term viability. The ultimate focus is to enhance the surface characteristics by employing stable functionalization of cell-interactive peptides or proteins.

\subsubsection{Surface chemistry/ECM studies on hMSC and hMSC-like cells}

Since cell behaviors are highly responsive to the surface chemistry, a considerable interest has been provided towards designing stable and functional biochemical environments. Several studies have employed ECM patterning to manipulate stem cell functions [84]. Changes in cell morphology/cell shape are shown to affect cytoskeletal stresses, polarity, cell survival, gene expression, migration and differentiation of stem cells $[85,86]$. The advances in micro-contact printing/photolithography have enabled patterning of variety of ECM patterns of varying resolution, geometry and regularity. 


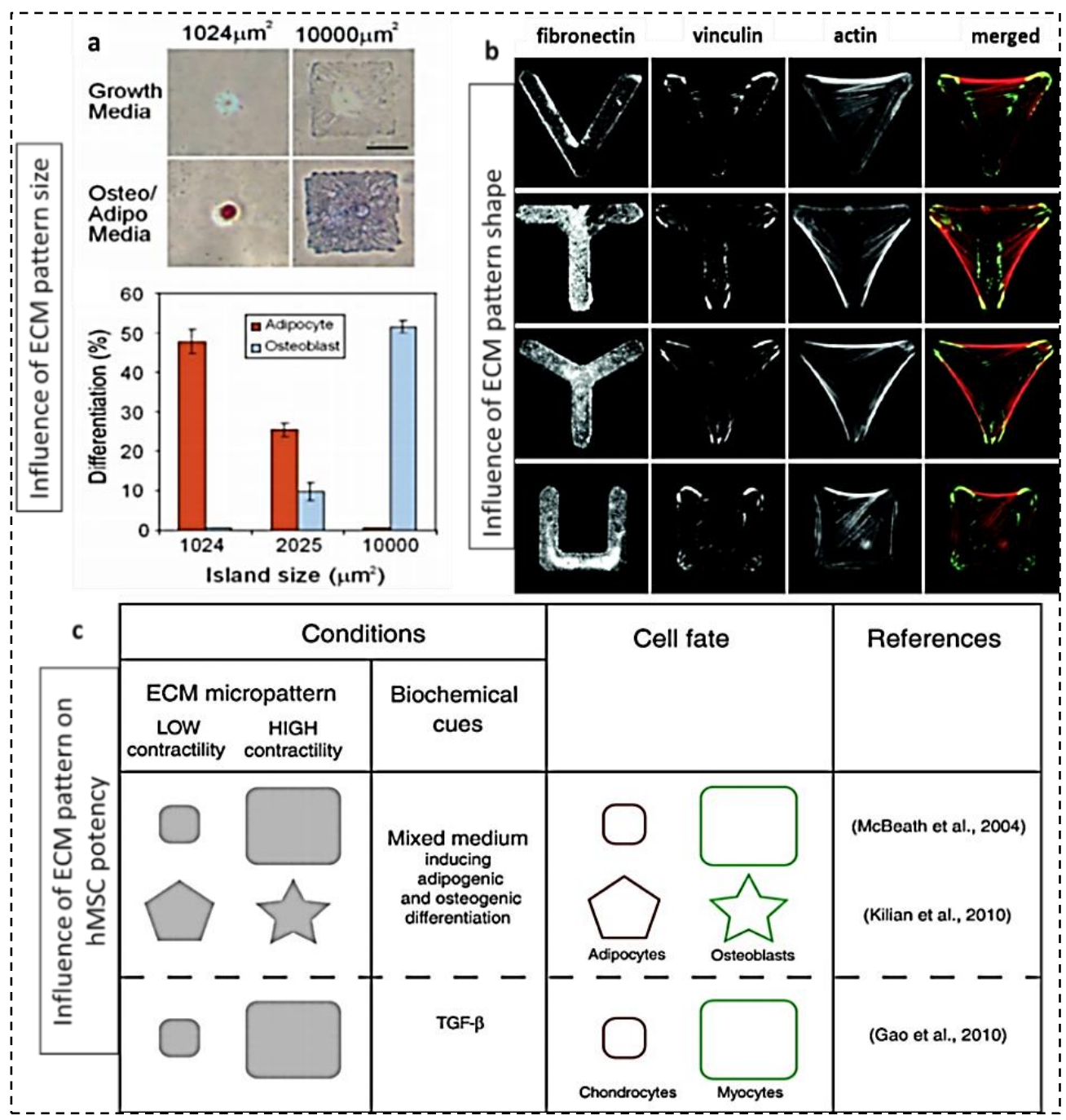

Figure 2-11: (a) F-actin stained images of hMSC cytoskeleton showing the effect of printed ECM area on hMSC shape and lineage. Adapted from ref. [94] (b) Influence of micro-printed fibronectin shapes on cell adhesion and distribution of cytoskeletal fibers of retinal epithelial cells. Adapted from ref.[95]. (c) Influences of ECM patterns in modulating cell morphology and cell fates. Adapted from ref.[92].

Micropatterning of ECM cues with different geometrical variations provides a reconstituted microenvironment unlike the traditional tissue culture plates in which micron scale effects of ECM adhesive patterns is hard to discern[85]. Traditional culture conditions also provide limited information due to spatial, chemical and mechanical constraints of the flat plastic/glass surfaces. In this regard, micropatterned ECM cues offer diverse matrix instructions which affect cell behaviors. 


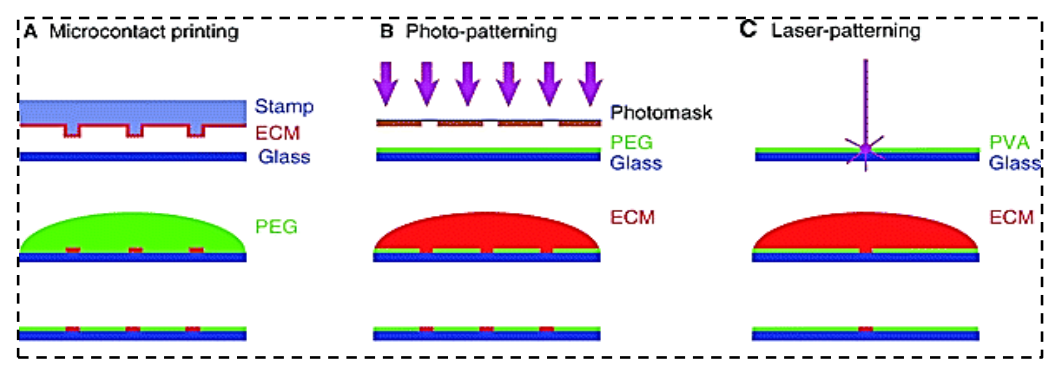

Figure 2-12: Micropatterning of glass or plastic culture substrates. Adapted from reference[85]

Patterns such as in Figure 2-11 are generated by various techniques of which one of the commonly employed method is micro-contact printing (Figure 2-12 a). This technique uses a PDMS stamp with patterned microscale features to imprint ECM proteins onto the culture substrate. Photopatterning (Figure 2-12 b) also offers convenience of studying diverse conditions with high resolution. This technique uses solutions selective photo-curing of the exposed regions which could be eventually coated with the ECM proteins. McBeath et.al, 2004 [87] used a micro-contact printing approach to create fibronectin islands of various sizes. They showed that the pattern shapes drive hMSC to either adipocyte or osteocyte commitment by altering the RhoA $\rightarrow$ ROCK signaling by changing the cytoskeletal tension. Another alternative but less popular method is laser patterning which involves precise cutting through polymeric microsections on glass followed by ECM protein coating (Figure 2-12 c). However, there are many practical limitations of this technique[85]. The pattern geometries affect the stresses on the cytoskeletal fibers in different ways which eventually directs respective stem cell fates. For instance, hMSCs in differentiating medium on $1000 \mu \mathrm{m}^{2}$ micropatterns showed lower degree of contractility and differentiate into adipocytes (fat cells). However, on larger area $\left(\sim 10,000 \mu \mathrm{m}^{2}\right)$ the cells are subjected to high contraction which induced osteogenic differentiation. More examples of shape and contractility regulation are summarized in Figure 2-11c. Studies by James et.al., 2008 [88] and Théry et.al., 2006 [89] show that cell contraction depends also on the convexity of cell edges. hMSCs grown on convex geometries, (E.g. pentagon), showed a tendency to differentiate into adipocytes, whereas on concave geometries, (E.g., star-shaped pattern) they differentiated into osteoblasts. These tendencies 
point to the importance of ECM protein patterns in mediating cell adhesion and cytoskeletal tensions which trigger specific cell responses. ECM platforms could applied to investigate stem cell behaviors and to solve challenges associated with cell expansion/differentiation in controlled environments. Lepperdinger et.al, 2014 [90] have reviewed various state-of-the art lab-on-a-chip technologies to facilitate precise monitoring of cell responses.

\subsubsection{Surface chemistry/ECM based platforms for neuronal cell investigations}

Neuronal cells are the most highly polarized cells in the body. Throughout their development, the neurons/neurites grow in their size and form new synapses but can never divide since they are differentiated from adult/neural stem cells[51]. During neuron growth, several biochemical components and ECM proteins regulate key cell behaviors [30, 53]. The key ECM proteins are Laminins, collagen IV, fibronectin, tenascins, netrins and proteoglycans[30]. Since the nature of ECM interactions with neurons resemble hMSC-ECM relationships, similar techniques of patterning neuronal ECM are employed to study neuronal responses to biochemical cues. 


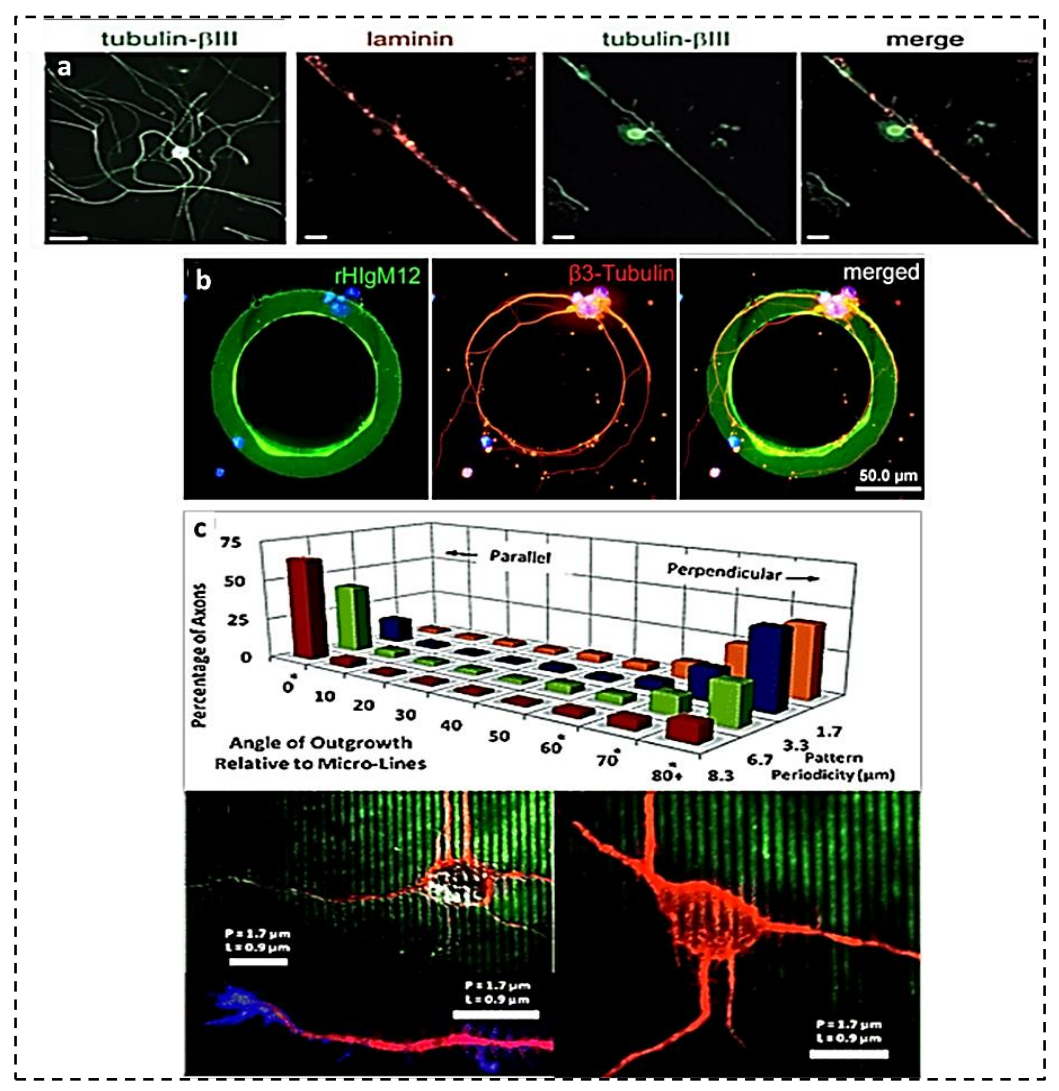

Figure 2-13: (a) Alignment of neuronal cell processes and cytoskeleton on laminin micropatterned lines, compared with the standard control (extreme left). Adapted from ref. [102] (b) Neurite alignment on recombinant IgG protein micropatterns. Adapted from ref.[103] (c) Cortical neuron behavior and axonogenesis controlled by periodicity of adhesive micro-lines of poly-l-lysine. Adapted from ref.[99].

A variety of ECM patterned matrices varying in periodicity[91], geometry[92] and shape alignments [93] are used to study the regulatory roles of ECM cues in modulating behaviors such as neuritogenesis, growth cone navigation and f-actin dynamics. ECM micropatterning could be effectively utilized for the exposure of specific ligands dendrites allowing for detailed investigations of in vivo mechanisms that determine axon specification and neuronal polarity[94]. Benzina et.al, 2014[95] showed that the micro-patterned substrate impacts the excitability of sensory neurons and promotes the activation of firing action potentials which were of similar nature as the motor neurons. A highly-controlled neurite direction and branching regulation is observed on their laminin microline printed substrates (Figure 2-13a). Shi et.al, 2007[96] and Oliva et.al, 2003[97] also showed the axon formation tendency on neurons cultured on laminin-1 coated micro-patterned lines. Similarly, Wheeler et.al, 1999 [98] and Kam et.al, 2001[99] also showed that a mixture of laminin and poly-lysine coated 
micro-lines regulated the formation of longer neurites especially axons. Shi et al., 2007 [96] also showed the correlation between length of the ECM coated lines with the length of neurites in which a direct proportionality was observed. Gupta et.al., 2010 showed that the laminin coated regions promoted centrosome orientation and subsequent growth of axons[94]. Apart from the natural ECM proteins, $X u$ et.al, 2013 [100] studied the influence of a human recombinant IgM protein in microenvironments of hippocampal neurons (Figure 2-13b). They showed that the protein could be successfully microprinted and promoted neuronal attachment and neurite growth in destined paths. Hart et.al, 2013[91] showed interesting correlation between axonal guidance and ECM micro-line periodicity in neurons (Figure 2-13c). They showed that the neurite direction underwent a transition from the regular parallel guidance direction to an unexpected perpendicular guidance when the gaps between the patterned lines were sufficiently narrow. This was attributed to the formation of growth cones which initiate neurite formations in perpendicular directions[91]. Stenger et.al, 1998 showed that continuous lines of SAM based amino-silane primer promoted neuronal maturation and axonogenesis compared to dashed/interrupted micropatterns[101]. These studies indicate how neurons sense the surrounding biochemical cues and undergo changes in their morphology and development.

\subsubsection{Traditional v/s SAM based approaches for ECM protein immobilization}

Although ECM protein micropatterning are used to study diverse cell-ECM protein interactions, most of the protein binding over the substrate of glass or tissue culture plates through physical binding or weak ionic interactions. This may cause non-uniform protein coating and may also be subjected to protein dislodgement and leaching with the progression of time. In the context of cell adhesion, it is desirable to have protein based platforms which can promote enhanced cell adhesion and an active cell-protein cross talk for successful proliferation and differentiation. However, the traditional methods such as physical adsorption, chemisorption or surface binding by ionic/Van der Waal's interactions have been shown to be unsuitable for both protein and cell immobilization [102]. Cell layers adhered on these substrates showed decreased adhesion and eventual detachment due to weak interaction of 
membrane proteins with poorly attached/randomly oriented protein layers. Hence, to improve surface reactivity and control the functionality, a well-defined chemical modification with a stable binding of proteins is desirable. There has been a growing interest to employ molecules that form self-assembled monolayers with various functional specificity which and cross reactivity with surfaces and the biomolecules[103].

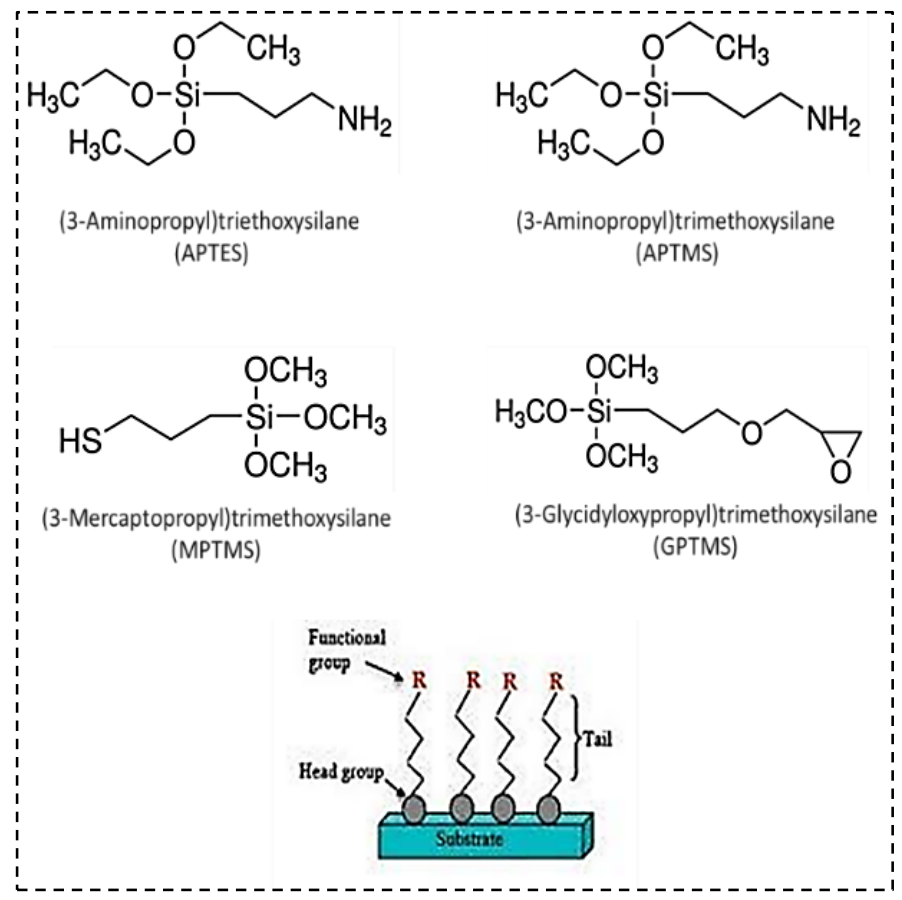

Figure 2-14: Organosilane molecules used to form Self assembled monolayers (SAM) based functionalization of cell/protein based substrates for enhanced reactivity. Adapted from reference [103]

Phillips et.al[104] illustrated using SAMs functionalized with four different functional groups, methyl $\left(-\mathrm{CH}_{3}\right)$, hydroxyl $(-\mathrm{OH})$, carboxyl $(-\mathrm{COOH})$ and amino $\left(-\mathrm{NH}_{2}\right)$, effected the adsorption of fibronectin onto the surfaces, which consequently allowed the osteogenic differentiation of hMSCs. The study illustrated that $-\mathrm{NH}_{2}$ allowed the highest level of osteogenic differentiation in osteoconductive medium, with up regulation of Runt-related transcription factor 2 (Runx2), BSP and osteocalcin (OCN) expression and alizarin red staining[104]. Similarly, Curran et.al,2006 [105] investigated methyl (-CH 3$)$, hydroxyl $(\mathrm{OH})$, carboxyl (-COOH), amino $\left(-\mathrm{NH}_{2}\right)$ and thiols (-SH) and also observed that hMSCs cultured on $-\mathrm{NH}_{2}$ surfaces showed a higher capability for osteogenic differentiation than other cell lineages or to remain in an 
undifferentiated state. Valamehr et.al, 2008[106] have also used the SAM technique to create different surface hydrophobicity and found the increase in surface hydrophilicities could increase hESC proliferation and differentiation.

With advances in bioelectronics and protein/peptide/gene based micro/nano-sensors, there is an increasing demand for well-defined and strong protein cross linkages to functionalize the surfaces[67]. Precursor molecules known as silanes, which can polymerize in aqueous solvents and form monolayers on the substrate surfaces were extensively used especially on silicon/silica based substrates. Typically, surface functionalization using silanes would involve the formation of stable silanol bonds on the underlying substrate while varying in the functionality of the exposed functional groups[107]. Commonly used silanes for covalent crosslinking of biomolecules ae shown in Figure 2-14. A variety of silane molecules have been utilized to create stronger protein linkages with the substrate compared to the traditionally used adsorption and deposition methods. Often, such strategies also involve the use of hetero or homo-bi-functional cross-linking agents such as glutaraldehyde, EDC and ester molecules. Hence for enhanced bioactivity and stable crosslinking of ECM proteins which is desirable in long term cell investigations, SAM based protein crosslinking could be a promising tool. The exact mechanisms and methods of silane based ECM protein linking and their consequences (results) would be discussed in detail the following chapters. 


\subsubsection{Role of ECM topography and mechanics in controlling key cell behaviors}

Cells sense the biophysical/mechanical factors such as stiffness, elasticity and substrate topography.
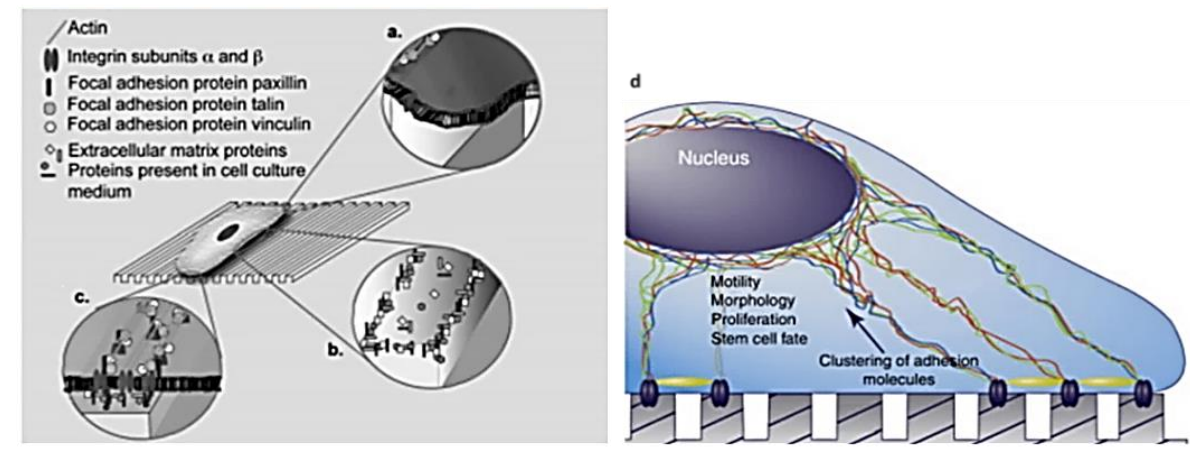

Figure 2-15: Sensing of substrate topography by membrane proteins and proteins involved in the formation of focal adhesion points at integrin binding sites. Adapted from ref. [117]. (d) Clustering of adhesion molecules and signaling to cell nucleus from focal adhesion points. Adapted from ref [21].

Substrate topography might involve several factors ranging from the nano/micron scale roughness profiles of the surfaces to the micro/nanopatterned physical features that are created on the surfaces by techniques such as soft lithography and laser guided lithography. Kulangara et.al,2009[108] reviewed the key factors and proteins involved in affecting the mechano-sensing of cell substrate topography (Figure 2-15 a). Most proteins that sense the topographical surroundings are present in the cell membrane and transmit the topographical signals to the nucleus via the cytoskeletal fibers[16, 17, 28] (Figure 2-15d). It is necessary to understand several topographical scenarios which affect cell adhesion[80]. The controlled response of stem cell towards topography has been effectively achieved through techniques such as electron beam lithography, colloidal/dip-pen lithography/nano-imprinting through which patterns with high precision and resolution are generated. In the context of neurons, several studies have indicated the topographical preferences of cell lines like PC12, neuroblastoma cells, neural stem cells and primary/progenitor neurons from cortical or hippocampal regions[67]. Apart from substrate topography, the substrate mechanics (rigidity, elastic modulus) and substrate roughness also influence cell behaviors. For instance, the ease 
of substrate stiffness manipulation by varying PDMS cross linking conditions has been utilized to demonstrate that lower substrate stiffness [<10:1] and higher roughness results in enhanced collagen production in hMSCs [74]. Highly rigid substrates (or low elastic modulus $\sim 0.5 \mathrm{kPa}$ ) support osteogenesis while at high elastic modulus there was increased occurrence of adipogenesis [111]. In case of neuron cells while softer substrates are known to support neurogenic differentiation in many studies, the relatively stiffer substrates are shown to enhance neuronal network activity $\left(\mathrm{Ca}^{2+}\right.$ oscillation) of cultured neuronal networks [129].

\subsubsection{Substrate topography and hMSC behaviors}

Since hMSCs are highly influenced by topographical variations, modulation of focal adhesion by surrounding topography has shown various behavioral and lineage consequences.

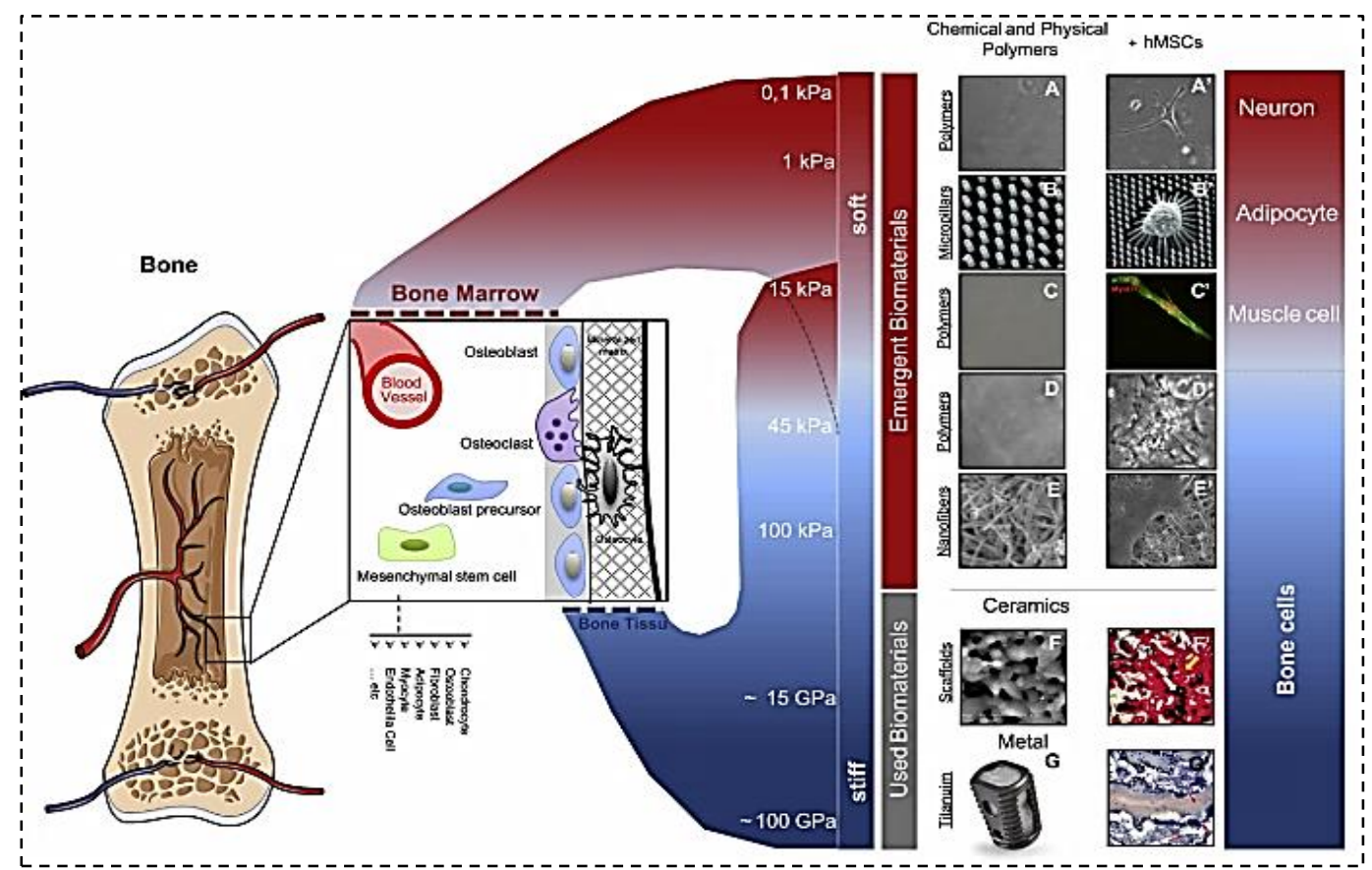

Figure 2-16: Schematic representation of bone marrow niche: (A-G) Differentiation towards specific lineages induced due to variations in material stiffness and topography. Adapted from reference [109]

Figure 2-16 summarizes the effect of topography, stiffness and nature of biomaterial on the lineage of bone marrow stem cells. Stiffness of substrate induces specific differentiation responses as shown by Engler et.al,2009[110] who cultured hMSCs on collagen based 
polyacrylamide hydrogels (without differentiation induction media). While soft gels the hMSCs showed neurogenic commitment, by slightly increasing the gel stiffness, the hMSCs displayed myogenic and osteogenic commitment. Zouani et al.,2012[111] showed that micropatterns of RGD ligand functionalized polyethylene which were exposed to UV radiation to create nano-pits of varying degree $(10 \mathrm{~nm}-100 \mathrm{~nm})$ that influenced hMSC adhesion and differentiation. Dalby et.al, 2007 [112] studied hMSC behavior on orderly/disorderly square and hexagonal patterns and nano-pits produced on polymethylmethacrylate (PMMA) substrates by electron beam lithography. Even with the absence of osteogenic medium, the disordered arrays induced enhanced osteogenesis.

Kilian et.al, 2010[113] showed that when hMSCs cultured on rectangular fibronectin islands of a constant area in aspect ratios (1:1, 3:2 and 4:1), the 4:1 substrates showed highest osteogenic differentiation (61\%), whereas the osteogenesis was hindered in 1:1 substrates. Leong et.al, 2007[114] showed that hMSCs cultured on nano-gratings on PDMS substrates showed alignment with cytoskeletal actin elongation which resulted in a significant upregulation of neuronal markers, demonstrating neurogenic commitment. Similarly, Tan et.al, 2010[115] cultured hMSCs on poly (lactic-co-glycolic acid) (PLGA) thin films with alternative adhesive and non-adhesive lanes of $20 \mathrm{~mm}$ and $40 \mathrm{~mm}$ widths. In this arrangement, osteogenesis was down-regulated while showing upregulation of myogenic and neurogenic genes. The myogenic commitment was prominent, and the presence of cardiac myosin heavy chain (MHC) protein suggested myocardial lineage specification. hMSCs cultured specifically on 70-100 nm nanotubes showed cytoskeletal stress and osteogenic commitment[116]. The surface topography also has significant effects on the nucleus profile, since the nuclear shape is distorted during cell extension and nucleus is mechanically integrated with the cytoskeleton. Such nuclear elongations have been correlated with osteoblastic differentiation[117].

\subsubsection{Substrate topography and Neuronal cell behaviors}

The biophysical niches of the neuronal microenvironments are extremely complex since the tissue architectures vary from different parts of the brain and due to changes in brain tissue 
mechanics during growth. Microfabricated physical environments for neuronal studies have been mostly performed on silicon or gold substrates. Since neurons are electrically active, these platforms are often integrated with sensor systems through which the electrophysiology of controlled set/network of neurons could be clearly investigated $[118,119]$.

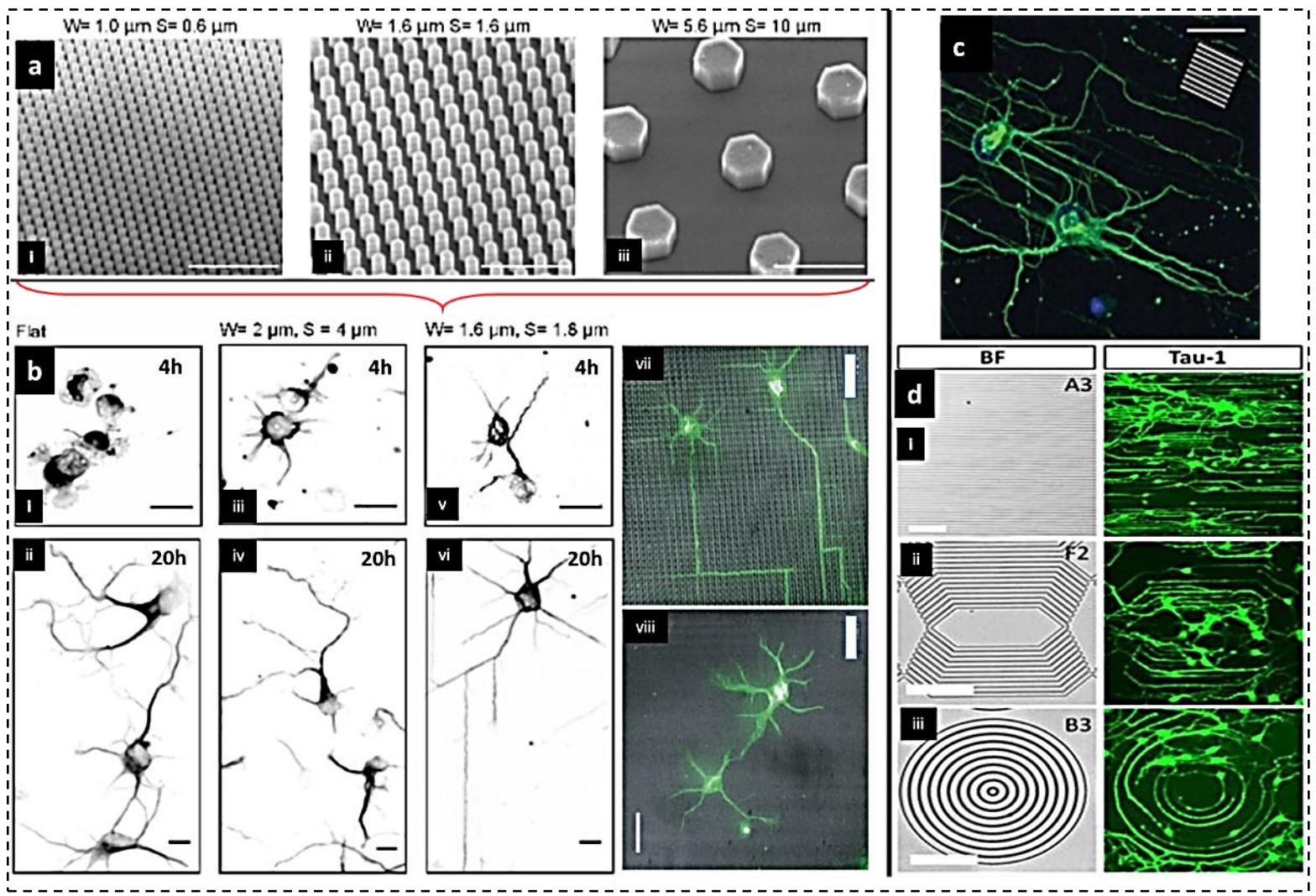

Figure 2-17: (a) Microfabrication of various PDMS based pillar architectures (b) (i-vi) Impact of pillar assemblies on the growth of nascent neurons. Comparison of neuron morphology on micropillars ( $v$-viii) and flat substrates (i and ii). Adapted from reference [129]. (c, d) neurite navigation of leech neurons and hippocampal neurons in micro-grooves. Adapted from references [130] and [131]. Scale bar: $10 \mu \mathrm{m}$

Micro-patterned cues in the range of $20 \mathrm{~nm}-20 \mu \mathrm{m}$ are shown to significantly affect neurite navigation, neurite length and polarity[120-122]. These platforms are either used to study early differentiation cues effect on neuronal stem cells/progenitor cells/ induced pleuripotent stem cells or to study nascent primary neurons in the context of cortical and hippocampal brain development[123]. Most studies point that neurons mostly sense anisotropic features like pillars in their vicinity and that the neuron growth cones navigate in the direction of pillars [124-126]. For instance, Micholt et.al, 2013 [127] found the importance of topographic 
signaling of mouse embryonic neurons where N-cadherin and golgi-centrosome complex was formed at a very early stage and caused budding of the earliest neurites towards the micropillar geometries. They showed that although $2 \mu \mathrm{m}$ width pillars (separated by $4 \mu \mathrm{m}$ ) supported efficient neurite navigation, the navigation was highly controlled and confined in orthogonal directions in closely knit $(0.6 \mu \mathrm{m})$ smaller pillars $(1 \mu \mathrm{m}$ width) (Figure 2-17 a, b (iii-viii). Further, compared to flat substrates (Figure 2-17 a, b (i, ii)) which mostly show neurites of equal lengths, these neurons in these microfeatures supported elongated neurite growth (Figure 2-17 b (v and vii)). Tonazzini et.al, 2013[128] studied the cytoskeleton arrangement and neurite growth of murine hippocampal neurons on microfabricated COC substrates with $1 \mu \mathrm{m}$ / $2 \mu \mathrm{m}$ grooves. They showed high preferential alignment of growth cones and confined navigation in $1 \mu \mathrm{m}$ and $2 \mu \mathrm{m}$ groove paths (Figure 2-17 c). Li et.al, 2015 [129] showed that the topographical parameters not only critically determine axonal/ dendrite length but also determine their branching (Figure 2-17 d).

Mefsin et.al, 2004 [120], created pillar arrays of $1 \mu \mathrm{m}$ height by photolithography in various widths and spacing. The main result of their finding is that the smallest inter-pillar gaps of 2 $\mu \mathrm{m}$ resulted in the longest neurite growth. Similarly, Hanson et.al 2008[130] developed conical PDMS textural cues of varying diameters and spacing by decal transfer lithography. They studied the effect on hippocampal neuron morphology and networking regularity over arrays ranging from 10-200 $\mu \mathrm{m}$ diameters and spacing. They showed that substrates with 10 $\mu \mathrm{m}$ diameter and $10 \mu \mathrm{m}$ spacing showed highest orthogonal confinement and regularity. As an alternative approach to both of these studies, the ability of line/pit based topography was also shown by Fozdar et.al, 2010[122]. They compared the ability of lines and holes of $300 \mathrm{~nm}$ and $2 \mu \mathrm{m}$ dimensions and showed that $>70 \%$ of neurons preferentially extended through holes rather than grooves with good axon formation. These platforms offer realistic understanding of material properties to be used as nerve guides or to correct permanent brain injuries, stroke and neurodegenerative diseases. 


\subsection{Design of in vitro platforms for study of specific cell functions}

As described in previous sections, routine in vivo cell and tissue investigation poses several practical difficulties. Moreover, traditional methods of cell culture (coverslips/standard flat plates) provide a rather artefactual environment. Hence the study of complex environments of cells such as hMSCs and nascent neurons needs demand highly realistic systems, which should be reproducible, reliable, robust and with minimal experimental inconsistencies. Further since most conventional bioassays are limited to endpoint detection, systems with realtime observation of dynamic cell mechanisms are desirable.

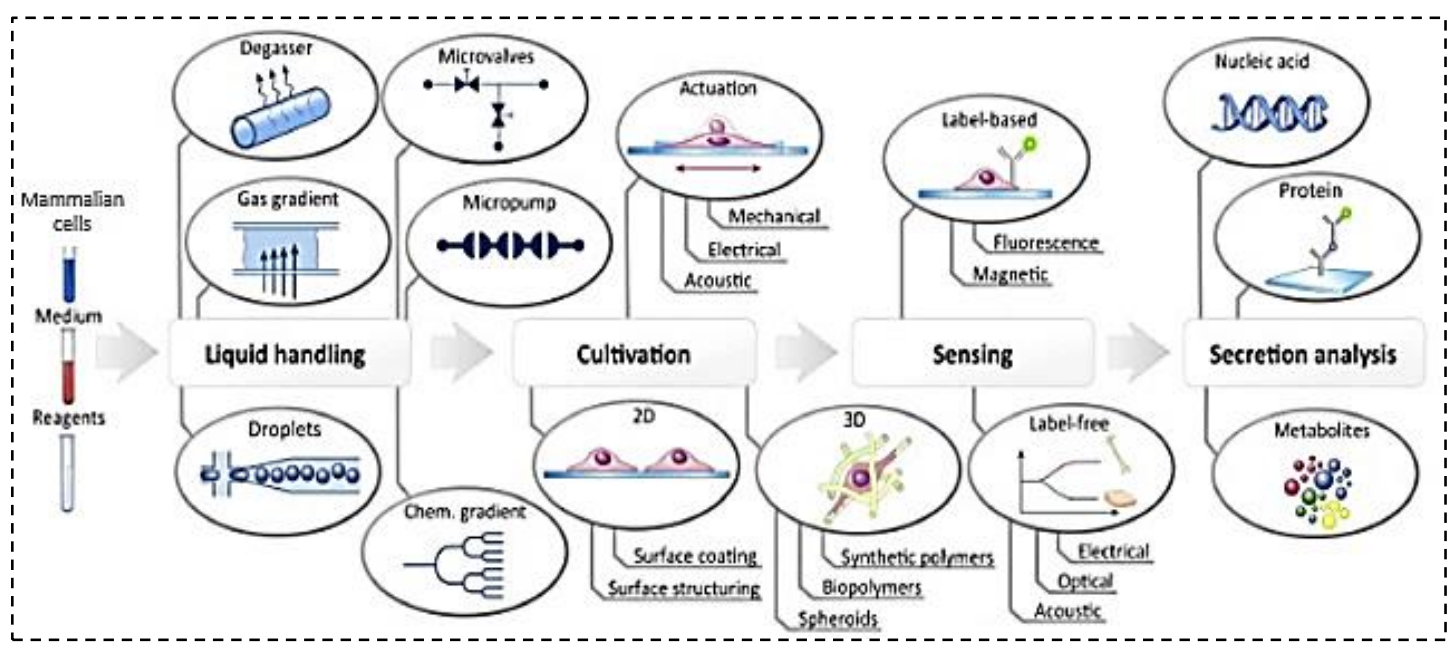

Figure 2-18: Schematic illustration of components and systems available to date that can be integrated into a fully automated lab-on-a-chip for stem cell analysis. Adapted from reference [98].

The advancement of micro/nanopatterning and lab-on-chip techniques could be aptly utilized to create realistic cell-ECM environments as well as dynamic observation of cell phenomena within miniaturized systems respectively [90] (Figure 2-18). In these systems, one of the key factors is to choose the right material and rely on right interface to mimic the natural bio architecture of the native tissues. Hence a variety of deformable and tunable materials such as PDMS, hydrogels, ceramics and polymers could be used with consideration of biochemical and biophysical requirements. The applications could be diverse ranging from fundamental studies of cell and tissue development/differentiation to the study of drug effects at cell/tissue levels, toxin screening, disease modeling and cell responses in response to diverse stimuli. 


\subsubsection{Differentiation/Tissue/Organ on-a-chip systems}

Animal experiments are time-consuming, expensive and controversial. Further the tissue functions inside the body are hard to decipher and need complex animal monitoring [67, 68].

The organs and tissues in body interact with each other and the metabolites released by an organ could closely affect the functioning of other organs. Alternative models which mimic the organ and tissue functions are needed which are both affordable and replicable. Integrated lab-on chip systems allow for miniaturization of the whole animal system and mimic the function of the human body or part of the body (organ/tissue/differentiated cell mass (E.g., spheroids))[68].

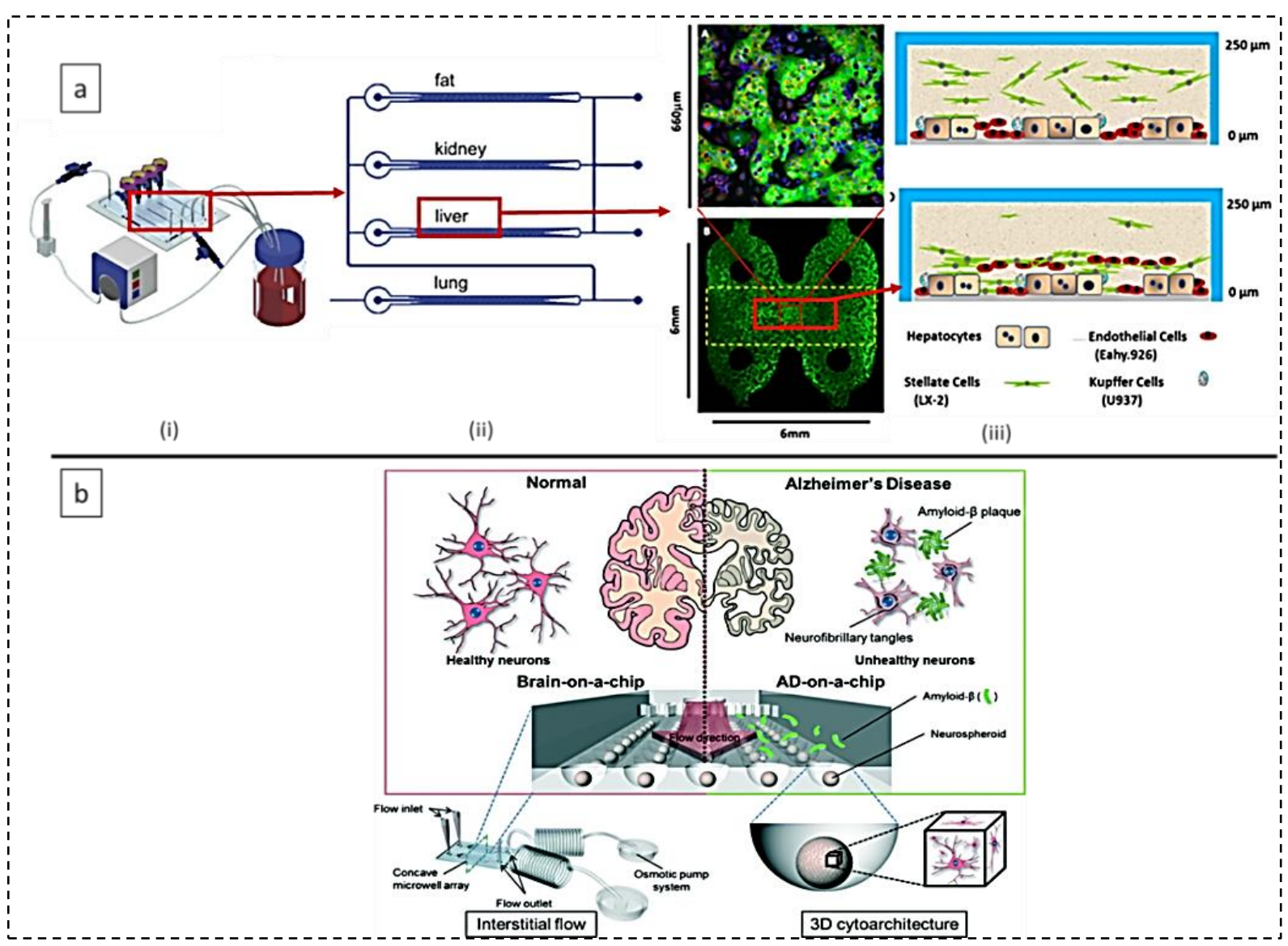

Figure 2-19: (a) Schematic illustration of multiple tissues on chip integrated system: (i) representation of fully integrated chip with medium inflow and metabolite outflow (ii) integration of different tissue types through microfluidic loop system (iii) design of liver niche with diverse cell types present in hepatic tissues [140, 141].(b) Alzheimers model on chip with neuronal 3D spheres. Adapted from reference [76] 
The concept of one of the initial integrated systems mimicking multiple tissues on chip were presented by Zhang et.al, 2009 [131](Figure 2-19 a). They created a multi-channel 3D microfluidic system with liver, lung, kidney and adipose cells and showed that the bio-activity of all the cells in the co-culture system were uncompromised. These systems can also be used to study differentiation models on chip. Tourovskaia et.al, 2005 [73] demonstrated a microfluidic system which could sustain myoblast cultures up to 2 weeks and supported their differentiation into myotubes. Ni et.al, 2009 [132] showed differentiation of hMSCs into adipocytes with cultures lasting for 2 weeks. These systems with perfusion controls could also be extended to more complex systems such as brain tissue and neuronal differentiation environments. Park et.al, 2009[72] have shown the differentiation of neuronal progenitor cells on chip across cytokine gradients and showed their differentiation into neurons. One of the practical miniaturized systems developed for in vitro modeling of Alzheimer's disease was recently developed by Park et.al, 2015[71] (Figure 2-19 b). They maintained osmotic fluid flow between 3D neurospheres and tested the toxic effect of amyloid- $\beta$ which is one of the major compounds causing Alzheimer's disease. They showed that amyloid- $\beta$ release through osmotic micropump significantly reduced the neurospheroid viability and impaired the neural networks, compared to the amyloid- $\beta$ treatment under static conditions. This system could be potentially used as in vitro brain model of neurodegenerative disease/drug screening [71]. Helm et.al, 2016[70] reviewed on-chip blood-brain barrier models with relevance to overall physiology and functions of brain. 


\subsubsection{PDMS as an in vitro study platform}

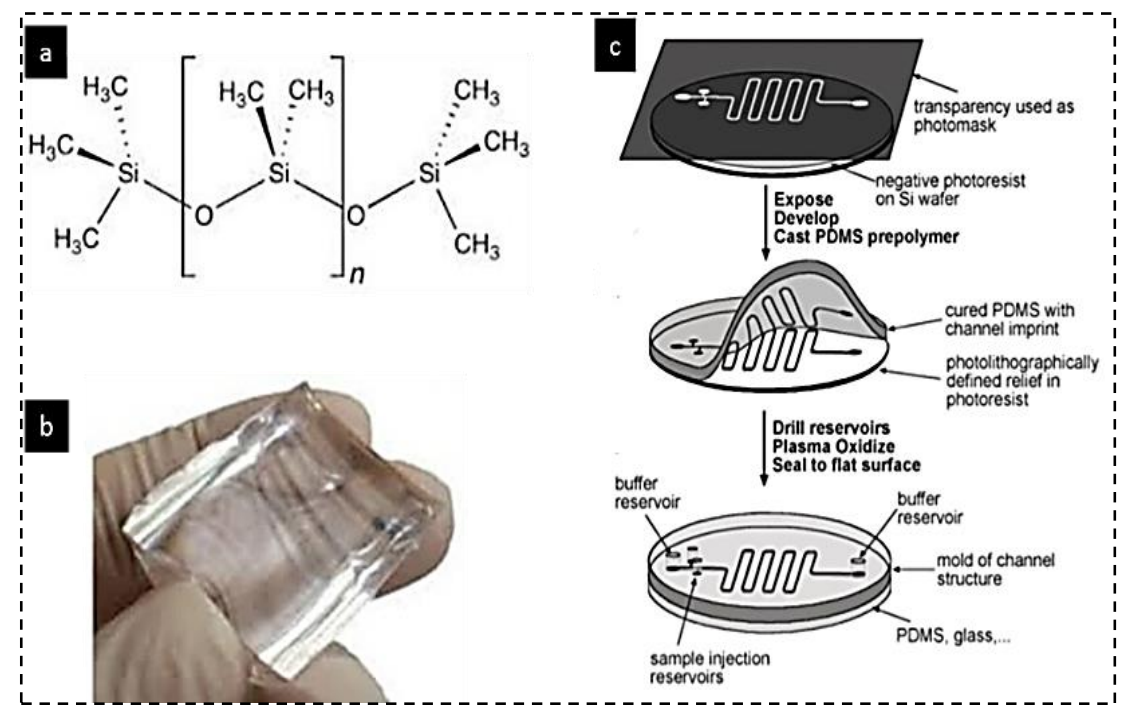

Figure 2-20: (a) Molecular structure of PDMS (b) photo image of thermo-cured PDMS (c) Replica molding and transfer of micro-features from photo-lithography derived silicon wafer to PDMS.

As a substrate material, PDMS (Figure 2-20 a, b) has shown to carry numerous properties that offer advantages over many other available biomaterials such as silicon, bio-glass and other polymers. For instance, an available master allows PDMS to be molded easily into sub-micron designs as defined by the master (replica molding) (Figure 2-20 c). Furthermore, the elastomeric properties of the PDMS can be easily tuned by the base: curing agents' ratio to cover a wide range of physiologically relevant elastic modulus for compared to other materials such as polyacrylamide gels, polyethylene glycol, and hyaluronan. The surface roughness of the PDMS can also be easily adjusted with different curing temperatures. The ease of molding to micron scale dimensions with high accuracy, optical transparency and low-cost fabrication are the criteria for choosing PDMS as material for hMSC and neuronal cell studies. Microfluidic technologies have been increasingly exploited to develop micro-devices for cell and tissue engineering application due to their precision in controlling the micro-environment with minimal bio-reagent consumption and experimental costs. While several bio-materials can be considered for the fabrication of micro-fluidic devices PDMS has been widely utilized for rapid prototyping due to their versatility, transparency, low costs and ease of fabrication. 


\subsection{Current challenges and missing links in in vitro cell studies}

Since hMSCs and neurons have complex in vivo niches, there are several parameters which eventually determine their adhesion and resulting cascade of cell processes such as proliferate, differentiation, polarity, formation of cell extensions (dendrites) and long term viably. To study the behaviors of these cells in response to specific biochemical and biophysical stimuli, it is desirable to create well-controlled reliable, reproducible, simple and versatile platforms. Several conventional methods involving the tissue plates/cover slip cell cultures offer limited understanding of cell functions and are prone to experimental errors. Further, these systems do not adequately emulate various in vivo complexities.

Although past study shows the influence of functional groups on mammalian cells, in case of mesenchymal stem cells only few studies show their behaviors towards surface functionality. Further the hMSC relationship with specific ECM proteins and ligands and their influence on their cytoskeletal dynamics and cell fates are not well established. Further, topographical evaluation of hMSC cell fate decision are only limited to 2D systems. Hence there is need for dynamic recreation of topographical microenvironments in combination with chemical cues and to evaluate these synergistic effects on simple and reliable micropatterned/ microfluidic platforms. Past studies related to nascent/developing body cells such as the neonatal and embryonic neuronal systems are also limited to coverslip/tissue plate based investigations which do not represent the formidable complexity of neuronal microenvironments. Developing neurons are highly sensitive to their surroundings. Chemical and physical aberrations in their ECM affect their polarity and complete differentiation. Although several techniques discuss ECM micropattern influences on neuron behavior and neurite alignment, they offer limited insight on neuronal cell adhesion behaviors and neurite lengths and branching in response to specific ECM cure. Conventional platforms also show transient/poor adhesion of ECM proteins which is a serious liaison towards long term neuronal cell investigation. With the rise of developmental studies within controlled microfluidic devices, such as the neurogenesis on 
chip, blood brain barrier on chip and brain on chip, there is a need to understand neuronal cell behavior in response to diverse ECM cues (surface chemistry, ECM protein and topography). Micro-fluidic technologies have been increasingly exploited to develop micro-devices for cell and tissue engineering application due to their precision in controlling the micro-environment with minimal bio-reagent consumption and experimental costs. While several bio-materials can be considered for the fabrication of micro-fluidic devices, poly (dimethylsiloxane) (PDMS) has been widely utilized for rapid prototyping in the exploratory stages of academic research due to their versatility, transparency, low costs and ease of fabrication [54]. However, the highly hydrophobic nature of PDMS surfaces often renders a non-biocompatible surface for long term cell analysis. While many have improved the bio-compatibility of native PDMS surface through oxygen plasma treatment to improve the surface wettability or to enhance cell adhesion, these effects are often transient. For example, hydrophobic recovery is often observed on PDMS surface and cell aggregation or clumping occurs on protein coated PDMS surface when the cell population confluences. These problems have limited the advancement of micro-fluidic technologies towards the development of micro-devices for long term cellular analysis. 
Table 1: Review of traditional and recent in vitro strategies to study cell-substrate interactions

\begin{tabular}{|c|c|c|c|c|}
\hline $\begin{array}{l}\text { Previously reported } \\
\text { polymeric cell culture } \\
\text { platforms }\end{array}$ & Chemical/physical modifications & Major advantages & Major disadvantages & Reference \\
\hline $\begin{array}{l}\text { PMMA/PS based micro- } \\
\text { structured devices }\end{array}$ & $\begin{array}{l}\rightarrow \text { Solvent assisted gluing } \\
\rightarrow \text { Melting/Lamination } \\
\rightarrow \text { Surface activation }\end{array}$ & $\begin{array}{l}\rightarrow \text { Short cycle time of production } \\
\rightarrow \text { Low material cost } \\
\rightarrow \text { Higher initial viability than } \\
\text { PDMS/PMMA in macroscopic cultures }\end{array}$ & $\begin{array}{l}\rightarrow \text { Expensive (High fabrication cost) } \\
\rightarrow \text { Low resolution }<40 \mu \mathrm{m} \\
\rightarrow \text { Translucent to light }\end{array}$ & [133-135] \\
\hline $\begin{array}{l}\text { Silicon/bio-glass } \\
\text { substrates }\end{array}$ & $\begin{array}{l}\rightarrow \text { Acid/plasma based oxidation } \\
\rightarrow \text { Nano-roughness modulation }\end{array}$ & $\begin{array}{l}\rightarrow \text { Biocompatible } \\
\rightarrow \text { Tolerant towards high temperature } \\
\text { and strong solvents }\end{array}$ & $\begin{array}{l}\rightarrow \text { Low optical transparency of silicon } \\
\rightarrow \text { High cost of fabrication } \\
\rightarrow \text { Complex micro scale machining } \\
\text { procedures of silicon/glass materials }\end{array}$ & {$[133,136]$} \\
\hline PMMA/Gold/silica/PDMS & $\begin{array}{l}\rightarrow \text { Direct SAM (self-assembled } \\
\text { monolayer) based modification with } \\
\text { organo-silanes with exposed reactive } \\
\text { functional groups }\end{array}$ & $\begin{array}{l}\rightarrow \text { Functional specificity } \\
\rightarrow \text { Higher reactivity with } \\
\text { cells/biomolecules } \\
\rightarrow \text { Controllability of deposition and } \\
\text { reaction parameters }\end{array}$ & $\begin{array}{l}\rightarrow \text { Low cell adhesion } \\
\rightarrow \text { Transient stability } \\
\rightarrow \text { Highly solvent dependent techniques } \\
\rightarrow \text { Poor cell sheet formation }\end{array}$ & [103-105] \\
\hline
\end{tabular}




\begin{tabular}{|c|c|c|c|c|}
\hline Glass/PDMS/PMMA/PCL & $\begin{array}{l}\rightarrow \text { Modification of polyelectrolytes by } \\
\text { layer-by-layer (LBL) controlled } \\
\text { deposition }\end{array}$ & $\begin{array}{l}\rightarrow \text { Controlled electrolytic adsorption } \\
\text { of the polyelectrolyte film up to nano- } \\
\text { scales } \\
\rightarrow \text { Can be also used on combinations } \\
\text { of polymers and particles or just with } \\
\text { mutually interacting nanoparticles }\end{array}$ & $\begin{array}{l}\rightarrow \text { High and unmodifiable surface } \\
\text { hardness of material surfaces } \\
\rightarrow \text { Cross-reactivity and non-specific } \\
\text { reactivity with medium serum proteins } \\
\text { and biomolecules } \\
\rightarrow \text { Only transient adsorption of the } \\
\text { electrostatic layer sustainable up to } \sim 48 \mathrm{~h}\end{array}$ & {$[135,137]$} \\
\hline
\end{tabular}




\begin{tabular}{|c|c|c|c|c|}
\hline Glass/quartz/PMMA/Silica & $\begin{array}{l}\rightarrow \text { ECM patterning by microcontact } \\
\text { printing } \\
\rightarrow \text { Photopatterning and laser patterning } \\
\text { of biomolecules }\end{array}$ & $\begin{array}{l}\rightarrow \text { Controllable resolution, patterning, } \\
\text { orientation and geometries (up to } \\
\text { nano-scale precision) } \\
\rightarrow \text { Proven capability in cell } \\
\text { mechanobiological studies }\end{array}$ & $\begin{array}{l}\rightarrow \text { Temporary modification (Not suitable } \\
\text { for long term cell investigation) } \\
\rightarrow \text { Expensive fabrication and sample } \\
\text { preparation procedures }\end{array}$ & $\begin{array}{l}{[81,85,} \\
139]\end{array}$ \\
\hline $\begin{array}{l}\text { PDMS/glass/silica/ } \\
\text { TCPs/Plastics }\end{array}$ & $\begin{array}{l}\rightarrow \text { Conventional of protein linking by } \\
\text { physical adsorption, chemisorption by } \\
\text { dip-coating or temperature based } \\
\text { settling mostly on plastic-like substrates } \\
\rightarrow \text { Feeder layer cell coating such as } \\
\text { Fibroblasts }\end{array}$ & $\begin{array}{l}\rightarrow \text { Surface binding by ionic and Van } \\
\text { der Waal's interactions } \\
\rightarrow \text { Relatively controllable and tunable } \\
\text { process compared to } \\
\text { random/uncontrolled modification } \\
\text { such as time/conc. optimized physical } \\
\text { adsorption }\end{array}$ & $\begin{array}{l}\rightarrow \text { Unsuitable for stable biomolecule } \\
\text { immobilization and subsequent adhesion } \\
\text { of cells } \\
\rightarrow \text { Protein conformation unknown } \\
\rightarrow \text { High chance for non-specific adsorption } \\
\text { on surfaces }\end{array}$ & {$[134,135]$} \\
\hline PLGA/PLCL/PLLA & $\begin{array}{l}\rightarrow \text { Poly (lactic-co-glycolic acid) (PLGA) } \\
\text { thin films } \\
\rightarrow \text { Coating with fibronectin }\end{array}$ & $\begin{array}{l}\rightarrow \text { Biodegradable and biocompatible } \\
\rightarrow \text { Can support the growth and } \\
\text { viability of hMSCs for } 8-14 \text { days } \\
\rightarrow \text { Moldable: E.g., alternative adhesive } \\
\text { and non-adhesive lanes/ } \\
\text { microfibers/nanofibers } \\
\rightarrow \text { Easy and low cost preparation }\end{array}$ & $\begin{array}{l}\rightarrow \text { Hydrophobic recovery on the surface } \\
\rightarrow \text { Low optical transparency (affects } \\
\text { experimental investigation) }\end{array}$ & {$[140,141]$} \\
\hline $\begin{array}{l}\text { PDMS/Glass/Gold/ } \\
\text { Hydrogel 2D casting }\end{array}$ & $\begin{array}{l}\rightarrow \text { Chemical patterning or bio- } \\
\text { patterning by dip pen lithography for } \\
\text { neuronal cell studies }\end{array}$ & $\begin{array}{l}\rightarrow \text { Neuronal cell morphological control } \\
\text { is achievable } \\
\rightarrow \text { High resolution }\end{array}$ & $\begin{array}{l}\rightarrow \text { Do not show long term implications on } \\
\text { neuron functions } \\
\rightarrow \text { Low degree of mechanical and surface } \\
\text { topographical tunability }\end{array}$ & $\begin{array}{l}{[94,99,} \\
142]\end{array}$ \\
\hline
\end{tabular}




\begin{tabular}{|c|c|c|c|c|}
\hline & & & $\rightarrow$ Viability only up to $5-6$ days & \\
\hline Glass/PMMA & $\begin{array}{l}\rightarrow \text { Electron beam lithography and nano- } \\
\text { imprinting for functional level hMSC cell } \\
\text { studies }\end{array}$ & $\begin{array}{l}\rightarrow \text { Controllable resolution } \\
\rightarrow \text { Induced osteogenesis without } \\
\text { needing osteogenic medium in hMSCs }\end{array}$ & $\begin{array}{l}\rightarrow \text { Chemical environments are not well } \\
\text { defined } \\
\rightarrow \text { Limited stiffness modulation } \\
\rightarrow \text { Expensive process }\end{array}$ & {$[143,144]$} \\
\hline $\begin{array}{l}\text { Native PDMS molding at } \\
\text { different stiffness }\end{array}$ & $\rightarrow$ Coating with fibronectin & $\begin{array}{l}\rightarrow \text { Moldable substrate elasticity } \\
\rightarrow \text { Support increasing cell growth up to } \\
7-9 \text { days in culture }\end{array}$ & $\begin{array}{l}\rightarrow \text { Hydrophobic recovery } \\
\rightarrow \text { Quick detachment of } 3 T 3 \text { fibroblasts, } \\
\text { MC3T3-E1, HUVECs and HeLa cells } \\
\rightarrow \text { Poor cell-cell contact } \\
\rightarrow \text { Inconsistent cell sheet formation and } \\
\text { beta catenin expression }\end{array}$ & $\begin{array}{l}{[81,139,} \\
145]\end{array}$ \\
\hline PMMA/glass & $\begin{array}{l}\rightarrow \text { Physical property modification to } \\
\text { induce random nano/micro scale } \\
\text { topography on the surfaces }\end{array}$ & $\begin{array}{l}\rightarrow \text { Optical transparency } \\
\rightarrow \text { Moderate biocompatibility }\end{array}$ & $\begin{array}{l}\rightarrow \text { Poor mimicking of multiscale ECM } \\
\text { physical environments } \\
\rightarrow \text { Needs expensive processing for } \\
\text { microfluidic implementation } \\
\rightarrow \text { Low gas exchange } \\
\rightarrow \text { Poor stiffness modulation }\end{array}$ & {$[134,146]$} \\
\hline PDMS/Glass/Gold & $\begin{array}{l}\rightarrow \text { Microfluidic adaptation to neuronal } \\
\text { studies by microfabrication/laser } \\
\text { cutting/EBL } \\
\rightarrow \text { Modification with plasma or plain }\end{array}$ & $\begin{array}{l}\rightarrow \text { Controlled confinement of neurons } \\
\rightarrow \text { Favorable biocompatibility and } \\
\text { electrophysiology of neurons }\end{array}$ & $\begin{array}{l}\rightarrow \text { Long term examination of neurons is } \\
\text { not possible } \\
\rightarrow \text { Serious bottleneck in long-term in vitro } \\
\text { studies concerning synaptogenesis, }\end{array}$ & {$[146,147]$} \\
\hline
\end{tabular}




\begin{tabular}{|c|c|c|c|c|}
\hline & poly-lysine or laminin coating & & $\begin{array}{l}\text { neural aging and death, synaptic plasticity, } \\
\text { and neural information processing }\end{array}$ & \\
\hline $\begin{array}{l}\text { Nano-fibrous aligned mats } \\
\text { (PLCL/PCL/PLGA) and } \\
\text { aligned (grooved } \\
\text { TCP/Glass features) }\end{array}$ & $\begin{array}{l}\rightarrow \text { Physical adsorption of proteins is } \\
\text { commonly used surface modification }\end{array}$ & $\begin{array}{l}\rightarrow \text { Control on cell alignments and cell } \\
\text { focal adhesion/integrin patterned } \\
\text { distribution } \\
\rightarrow \text { Precise modulation of cytoskeletal } \\
\text { stresses }\end{array}$ & $\begin{array}{l}\rightarrow \text { Hard to introduce microscale large } \\
\text { features such as holes and pillars of } \\
\text { precise geometry and with varying } \\
\text { stiffness } \\
\rightarrow \text { May involve difficulties in imaging and } \\
\text { laborious processing }\end{array}$ & $\begin{array}{l}{[85,145,} \\
148]\end{array}$ \\
\hline $\begin{array}{l}\text { 2D Flat TCP cultures /bio- } \\
\text { glass and glass cover-slips }\end{array}$ & $\begin{array}{l}\rightarrow \text { With/without chemical and } \\
\text { biomolecular modifications } \\
\rightarrow \text { Without physical/topographical } \\
\text { modifications }\end{array}$ & $\begin{array}{l}\rightarrow \text { Standard culture methods for most } \\
\text { of the cell and biomolecular property } \\
\text { analysis } \\
\rightarrow \text { Good repeatability of cell functions }\end{array}$ & $\begin{array}{l}\rightarrow \text { Does not reflect osteogenic or } \\
\text { neurogenic micro and nano-features } \\
\rightarrow \text { Cannot be used in stimuli responsive } \\
\text { cell studies against electrical/mechanical } \\
\text { stimuli }\end{array}$ & $\begin{array}{l}{[135]} \\
\end{array}$ \\
\hline $\begin{array}{l}\text { PS-microwells and PDMS- } \\
\text { Sylgard } 184\end{array}$ & $\begin{array}{l}\rightarrow \text { No treatment or treatment with } \\
\text { gelatin/alginate/matrigels to form thin } \\
\text { film of biocompatible gels }\end{array}$ & $\begin{array}{l}\rightarrow \text { Maintains cell health and promotes } \\
\text { cell differentiation upon the success of } \\
\text { long term culture }\end{array}$ & $\begin{array}{l}\rightarrow \text { Low hMSC proliferation } \\
\rightarrow \text { Gel instability and coating } \\
\text { inconsistencies are common } \\
\rightarrow \text { Hard to manipulate biophysical } \\
\text { parameters of the substrates } \\
\rightarrow \text { Interference by serum protein layer and } \\
\text { protein corona formation on surfaces due } \\
\text { to surface charge electrostatic interactions }\end{array}$ & {$[149,150]$} \\
\hline $\begin{array}{l}\text { Glass coverslips and silica } \\
\text { used in neuronal cell } \\
\text { studies }\end{array}$ & $\begin{array}{l}\rightarrow \text { Treatment with Poly-D-Lysine or } \\
\text { laminin-I or L-ornithine without plasma }\end{array}$ & $\begin{array}{l}\rightarrow \text { Supports initial attachment of } \\
\text { primary neurons and proliferation of } \\
\text { neuronal progenitor embryonic cells }\end{array}$ & $\begin{array}{l}\rightarrow \text { Steady decline in cellular responses } \\
\text { over time } \\
\rightarrow \text { Cell clumping over time }\end{array}$ & $\begin{array}{l}\text { [81,139, } \\
151]\end{array}$ \\
\hline
\end{tabular}




\begin{tabular}{|l|l|l|l|}
\hline & & & $\begin{array}{l}\rightarrow \text { Substrate stiffness contrasts the } \\
\text { relatively soft and gel like neurogenic } \\
\text { tissue environments }\end{array}$ \\
\hline PC/PMMA/COC/PS/PDMS & $\begin{array}{l}\rightarrow \text { UV-ozone for 15-60 mins or oxygen } \\
\text { plasma for 30- } \\
120 \text { s. }\end{array}$ & $\begin{array}{l}\rightarrow \text { PDMS supported highest gas } \\
\text { permeability and biocompatibility } \\
\text { compared to other substrates. } \\
\rightarrow \text { Order of gas permeability: } \\
\text { PDMS }>\text { COC > PC > PS > PMMA } \\
\rightarrow \text { Order of biocompatibility: } \\
\text { PDMS }>\text { PC }>\text { COC }>\text { PS }>\text { PMMA }\end{array}$ & $\begin{array}{l}\rightarrow \text { Hydrophobic recovery within 4 weeks } \\
\text { after treatment }\end{array}$ \\
$\begin{array}{l}\rightarrow \text { Cell death highest in PMMA followed by } \\
\text { PS, COC and PC substrates }\end{array}$ & $\begin{array}{l}\text { [81, 134, } \\
139]\end{array}$ \\
\hline
\end{tabular}




\subsection{Summary}

Cell surface interactions are critical for survival, differentiation and overall expression of both differentiating cells and young differentiating cells which have significant relevance in tissue engineering and cell developmental studies. In vivo conditions are extremely complex with a diverse mélange of growth factors, matrix proteins, ions and signaling molecules influence cell functions. Cells sense the chemical and physical cues in their immediate ECM and their genes react in appropriate way to surrounding signals. Hence the cell behavior can be manipulated by modulating the surface chemistry/ topography of the material surfaces.

hMSCs are increasingly used for stem cell based therapies and in tissue engineering owing to their abundance, ease of isolation, multipotency and non-immunogenic properties. hMSCs respond to the biochemical cues (E.g., ECM protein composition) and biophysical cues (E.g. topography) by modulation of their cell cytoskeleton through integrin mediated signaling. This property has been extensively used to design suitable regenerative therapies and biomaterials which can promote integrin mediated focal adhesion formation and activation of hMSC cytoskeleton. The hMSCs cytoskeletal system not only responds to ECM proteins/ topography on the biomaterial surface but also results in the activation of pathways which induce hMSC differentiation by communicating these signals to the cell nucleus.

Akin to hMSCs, neuronal cells thrive in complex environment of the brain. The developing neurons at their early and nascent stage possess similar growth and migration characteristics as hMSCs. The composition of neuronal ECM is like hMSC environments except for few proteins. Past studies have also shown the active inter-talk between hMSC released growth factors and their positive implication on neuronal growth and viability. Further the extracellular biochemical and biophysical environments of nascent neurons could be adequately manipulated and tuned to morphologically regulate their adhesion, neuritogenesis, branching, and networking. In recent 
years, advances in micro and nanofabrication and chemical crosslinking techniques have enabled precise modulation of material dynamics and material biochemical composition, surface roughness and topographical featuring with high precision. The congenial properties of PDMS and the transferability and tunability of micro/nanofeatures on PDMS surface has led to several cells based investigation especially in miniaturized platforms due to several inherent practical experimental advantages. This stirs the use, manipulation and improvement of PDMS surface properties towards investigation of cell material interactions towards several research questions relevant in hMSC based tissue engineering and biochemical and biophysical tuning of neuronal development. However, PDMS surfaces are extremely hydrophobic and are also prone to some limitations in terms of designing suitable chemical/ topographic features on its surface. Hence there is a clear need to develop versatile strategies to improve PDMS surface reactivity and tunability of surface features for well-defined studies on biochemical and biophysical modulation of key stem cell fates. 


\section{References}

1. Knisely, K. and S.F. Gilbert, Developmental Biology 8th edition ed. 2009.

2. Thomson, J.A., et al., Embryonic stem cell lines derived from human blastocysts. Science, 1998. 282(5391): p. 1145-1147.

3. Rudel, D. and R.J. Sommer, The evolution of developmental mechanisms. Developmental Biology, 2003. 264(1): p. 15-37.

4. $\quad \mathrm{R}, \mathrm{S}$., The relationship between the spleen colony-forming cell and the haemopoietic stem cell. Blood cells 1978. 4: p. 7-25.

5. Jones, D.L. and A.J. Wagers, No place like home: anatomy and function of the stem cell niche. Nature Reviews Molecular Cell Biology, 2008. 9(1): p. 11-21.

6. Iozzo, R.V. and A.D. Murdoch, Proteoglycans of the extracellular environment: Clues from the gene and protein side offer novel perspectives in molecular diversity and function. Faseb Journal, 1996. 10(5): p. 598-614.

7. Jarvelainen, $H_{\text {., }}$ et al., Extracellular Matrix Molecules: Potential Targets in Pharmacotherapy. Pharmacological Reviews, 2009. 61(2): p. 198-223.

8. Frantz, C., K.M. Stewart, and V.M. Weaver, The extracellular matrix at a glance. Journal of Cell Science, 2010. 123(24): p. 4195-4200.

9. Takagi, J., Structural basis for ligand recognition by RGD (Arg-Gly-Asp)-dependent integrins. Biochemical Society Transactions, 2004. 32: p. 403-406.

10. Kao, W.Y.J., Evaluation of protein-modulated macrophage behavior on biomaterials: designing biomimetic materials for cellular engineering. Biomaterials, 1999. 20(23-24): p. 2213-2221.

11. Timpl, R., et al., Structure and function of laminin LG modules. Matrix Biology, 2000. 19(4): p. 309-317.

12. Harvey, S.J. and J.H. Miner, Revisiting the glomerular charge barrier in the molecular era. Current Opinion in Nephrology and Hypertension, 2008. 17(4): p. 393-398.

13. Morita, H., et al., Heparan sulfate of perlecan is involved in glomerular filtration. Journal of the American Society of Nephrology, 2005. 16(6): p. 1703-1710.

14. Rozario, T. and D.W. DeSimone, The extracellular matrix in development and morphogenesis: A dynamic view. Developmental Biology, 2010. 341(1): p. 126-140.

15. Taipale, J. and J. KeskiOja, Growth factors in the extracellular matrix. Faseb Journal, 1997. 11(1): p. 51-59.

16. Aplin, A.E. and R.L. Juliano, Integrin and cytoskeletal regulation of growth factor signaling to the MAP kinase pathway. Journal of Cell Science, 1999. 112(5): p. 695-706.

17. Howe, A., et al., Integrin signaling and cell growth control. Current Opinion in Cell Biology, 1998. 10(2): p. 220-231.

18. OrtíZ, A., et al., Fibronectin (FN) decreases glomerular lesions and synthesis of tumour necrosis factor-alpha (TNF- $\alpha$ ), platelet-activating factor (PAF) and FN in proliferative glomerulonephritis. Clinical \& Experimental Immunology, 1995. 101(2): p. 334-340.

19. Adams, J.C. and F.M. Watt, Regulation of Development and Differentiation by the Extracellular-Matrix. Development, 1993. 117(4): p. 1183-1198.

20. Vainio, S. and U. Muller, Inductive tissue interactions, cell signaling, and the control of kidney organogenesis. Cell, 1997. 90(6): p. 975-978.

21. Rosso, F., et al., From cell-ECM interactions to tissue engineering. Journal of Cellular Physiology, 2004. 199(2): p. 174-180. 
22. Harburger, D.S. and D.A. Calderwood, Integrin signalling at a glance (vol 122, pg 159, 2009). Journal of Cell Science, 2009. 122(9): p. 1472-1472.

23. Leitinger, B. and E. Hohenester, Mammalian collagen receptors. Matrix Biology, 2007. 26(3): p. 146-155.

24. Humphries, J.D., A. Byron, and M.J. Humphries, Integrin ligands at a glance. Journal of Cell Science, 2006. 119(19): p. 3901-3903.

25. Xian, X.J., S. Gopal, and J.R. Couchman, Syndecans as receptors and organizers of the extracellular matrix. Cell and Tissue Research, 2010. 339(1): p. 31-46.

26. Jinka, R., et al., Alterations in Cell-Extracellular Matrix Interactions during Progression of Cancers. International Journal of Cell Biology, 2012. 2012: p. 8.

27. Stewart, D.A., C.R. Cooper, and R.A. Sikes, Changes in extracellular matrix (ECM) and ECM-associated proteins in the metastatic progression of prostate cancer. Reproductive Biology and Endocrinology, 2004. 2(1): p. 2.

28. Domínguez-Giménez, P., N.H. Brown, and M.D. Martín-Bermudo, Integrin-ECM interactions regulate the changes in cell shape driving the morphogenesis of the $<e m>$ Drosophila</em> wing epithelium. Journal of Cell Science, 2007. 120(6): p. 10611071.

29. Kinney, M.A. and T.C. McDevitt, Emerging strategies for spatiotemporal control of stem cell fate and morphogenesis. Trends in Biotechnology, 2013. 31(2): p. 78-84.

30. Schmid, R.S. and E.S. Anton, Role of integrins in the development of the cerebral cortex. Cerebral Cortex, 2003. 13(3): p. 219-224.

31. Magdaleno, S.M. and T. Curran, Brain development: Integrins and the Reelin pathway. Current Biology, 2001. 11(24): p. R1032-R1035.

32. Sharpe, M. and N. Mount, Genetically modified T cells in cancer therapy: opportunities and challenges. Disease Models \& Mechanisms, 2015. 8(4): p. 337-350.

33. Houghton, A.N. and J.A. Guevara-Patino, Immune recognition of self in immunity against cancer. Journal of Clinical Investigation, 2004. 114(4): p. 468-471.

34. Sagar, J., et al., Role of stem cells in cancer therapy and cancer stem cells: a review. Cancer Cell International, 2007. 7.

35. Siniscalco, D., et al., Mesenchymal stem cells in treating autism: Novel insights. World Journal of Stem Cells, 2014. 6(2): p. 173-178.

36. Cigognini, D., et al., Engineering in vitro microenvironments for cell based therapies and drug discovery. Drug Discovery Today, 2013. 18(21-22): p. 1099-1108.

37. Robertson, J.A., Embryo Stem Cell Research: Ten Years of Controversy. Journal of Law Medicine \& Ethics, 2010. 38(2): p. 191-+.

38. De Feo, D., et al., Neural stem cell transplantation in central nervous system disorders: from cell replacement to neuroprotection. Current Opinion in Neurology, 2012. 25(3): p. 322-333.

39. Ladran, I., et al., Neural stem and progenitor cells in health and disease. Wiley Interdisciplinary Reviews-Systems Biology and Medicine, 2013. 5(6): p. 701-715.

40. Bunnell, B.A., et al., Adipose-derived stem cells: Isolation, expansion and differentiation. Methods, 2008. 45(2): p. 115-120.

41. Vellasamy, S., et al., Isolation and characterisation of mesenchymal stem cells derived from human placenta tissue. World Journal of Stem Cells, 2012. 4(6): p. 53-61.

42. Secunda, R., et al., Isolation, expansion and characterisation of mesenchymal stem cells from human bone marrow, adipose tissue, umbilical cord blood and matrix: a comparative study. Cytotechnology, 2015. 67(5): p. 793-807. 
43. Zhang, X., et al., Isolation and Characterization of Mesenchymal Stem Cells From Human Umbilical Cord Blood: Reevaluation of Critical Factors for Successful Isolation and High Ability to Proliferate and Differentiate to Chondrocytes as Compared to Mesenchymal Stem Cells From Bone Marrow and Adipose Tissue. Journal of Cellular Biochemistry, 2011. 112(4): p. 1206-1218.

44. Gronthos, S., et al., A method to isolate and culture expand human dental pulp stem cells. Methods Mol Biol, 2011. 698: p. 107-21.

45. DiMarino, A.M., A.I. Caplan, and T.L. Bonfield, Mesenchymal Stem Cells in Tissue Repair. Frontiers in Immunology, 2013. 4: p. 201.

46. Lutolf, M.P. and J.A. Hubbell, Synthetic biomaterials as instructive extracellular microenvironments for morphogenesis in tissue engineering. Nat Biotech, 2005. 23(1): p. 47-55.

47. Legate, K.R., S.A. Wickstrom, and R. Fassler, Genetic and cell biological analysis of integrin outside-in signaling. Genes \& Development, 2009. 23(4): p. 397-418.

48. Buitenhuis, M., The role of PI3K/protein kinase $B(P K B / c-a k t)$ in migration and homing of hematopoietic stem and progenitor cells. Current Opinion in Hematology, 2011. 18(4): p. 226-230.

49. Kanatsu-Shinohara, M., et al., Homing of mouse spermatogonial stem cells to germline niche depends on beta1-integrin. Cell Stem Cell, 2008. 3(5): p. 533-42.

50. Saladin, K., Anatomy \& Physiology The Unity of Form and Function. 2011.

51. Alvarez-Buylla, A. and J.M. Garcia-Verdugo, Neurogenesis in adult subventricular zone. Journal of Neuroscience, 2002. 22(3): p. 629-634.

52. Pencea, V., et al., Neurogenesis in the subventricular zone and rostral migratory stream of the neonatal and adult primate forebrain. Experimental Neurology, 2001. 172(1): p. 116.

53. Florio, M. and W.B. Huttner, Neural progenitors, neurogenesis and the evolution of the neocortex. Development, 2014. 141(11): p. 2182-2194.

54. Lui, J.H., D.V. Hansen, and A.R. Kriegstein, Development and Evolution of the Human Neocortex. Cell, 2011. 146(1): p. 18-36.

55. Stead, J.D.H., et al., Transcriptional profiling of the developing rat brain reveals that the most dramatic regional differentiation in gene expression occurs postpartum. Journal of Neuroscience, 2006. 26(1): p. 345-353.

56. Aung, K.H., et al., Role of Environmental Chemical Insult in Neuronal Cell Death and Cytoskeleton Damage. Biological \& Pharmaceutical Bulletin, 2015. 38(8): p. 1109-1112.

57. Bian, S., Neural Stem Cells - New Perspectives. Cell Adhesion Molecules in Neural Stem Cell and Stem Cell- Based Therapy for Neural Disorders, ed. L. Bonfanti. 2013.

58. Barros, C.S., S.J. Franco, and U. Muller, Extracellular Matrix: Functions in the Nervous System. Cold Spring Harbor Perspectives in Biology, 2011. 3(1).

59. Kazanis, I. and C. Ffrench-Constant, Extracellular Matrix and the Neural Stem Cell Niche. Developmental Neurobiology, 2011. 71(11): p. 1006-1017.

60. Staquicini, F.I., et al., Discovery of a functional protein complex of netrin-4, laminin gamma1 chain, and integrin alpha6beta1 in mouse neural stem cells. Proc Natl Acad Sci U S A, 2009. 106(8): p. 2903-8.

61. Geissler, M., et al., Primary Hippocampal Neurons, Which Lack Four Crucial Extracellular Matrix Molecules, Display Abnormalities of Synaptic Structure and Function and Severe Deficits in Perineuronal Net Formation. Journal of Neuroscience, 2013. 33(18): p. 7742-+. 
62. Dietzel, I. and U. Heinemann, Dynamic Variations of the Brain-Cell Microenvironment in Relation to Neuronal Hyperactivity. Annals of the New York Academy of Sciences, 1986. 481: p. 72-86.

63. Langer, R. and J. Vacanti, Tissue engineering. Science, 1993. 260(5110): p. 920-926.

64. Ma, X.Y., et al., Deterministically patterned biomimetic human iPSC-derived hepatic model via rapid $3 D$ bioprinting. Proceedings of the National Academy of Sciences of the United States of America, 2016. 113(8): p. 2206-2211.

65. ANENE-NZELU, C., et al., LIVER TISSUE MODEL FOR DRUG TOXICITY SCREENING. Journal of Mechanics in Medicine and Biology, 2011. 11(02): p. 369-390.

66. Ma, C., et al., On-Chip Construction of Liver Lobule-like Microtissue and Its Application for Adverse Drug Reaction Assay. Analytical Chemistry, 2016. 88(3): p. 1719-1727.

67. Ali Khademhosseini, J.B., Mehmet Toner, Shuichi Takayama, Micro and Nano engineering of the cell microenvironment: Technologies and Applications. 2008.

68. Bhatia, S.N. and D.E. Ingber, Microfluidic organs-on-chips. Nature Biotechnology, 2014. 32(8): p. 760-772.

69. Edmondson, R., et al., Three-Dimensional Cell Culture Systems and Their Applications in Drug Discovery and Cell-Based Biosensors. Assay and Drug Development Technologies, 2014. 12(4): p. 207-218.

70. van der Helm, M.W., et al., Microfluidic organ-on-chip technology for blood-brain barrier research. Tissue Barriers, 2016. 4(1).

71. Park, J., et al., Three-dimensional brain-on-a-chip with an interstitial level of flow and its application as an in vitro model of Alzheimer's disease. Lab on a Chip, 2015. 15(1): p. 141150.

72. Park, J.Y., et al., Differentiation of Neural Progenitor Cells in a Microfluidic ChipGenerated Cytokine Gradient. Stem Cells, 2009. 27(11): p. 2646-2654.

73. Tourovskaia, A., X. Figueroa-Masot, and A. Folch, Differentiation-on-a-chip: A microfluidic platform for long-term cell culture studies. Lab on a Chip, 2005. 5(1): p. 14-19.

74. Chuah, Y.J., et al., Combinatorial effect of substratum properties on mesenchymal stem cell sheet engineering and subsequent multi-lineage differentiation. Acta Biomaterialia, 2015. 23: p. 52-62.

75. Carroll, M.J., L.E. Stopfer, and P.K. Kreeger, A simplified culture system to examine soluble factor interactions between mammalian cells. Chemical Communications, 2014. 50(40): p. 5279-5281.

76. Chen, Y.C., et al., Single cell dual adherent-suspension co-culture micro-environment for studying tumor-stromal interactions with functionally selected cancer stem-like cells. Lab on a Chip, 2016. 16(15): p. 2935-2945.

77. Bacakova, L., et al., Modulation of cell adhesion, proliferation and differentiation on materials designed for body implants. Biotechnology Advances, 2011. 29(6): p. 739-767.

78. Keselowsky, B.G., D.M. Collard, and A.J. Garcia, Surface chemistry modulates focal adhesion composition and signaling through changes in integrin binding. Biomaterials, 2004. 25(28): p. 5947-5954.

79. Dowling, D.P., et al., Effect of Surface Wettability and Topography on the Adhesion of Osteosarcoma Cells on Plasma-modified Polystyrene. Journal of Biomaterials Applications, 2011. 26(3): p. 327-347.

80. Wheeldon, I., et al., Nanoscale tissue engineering: spatial control over cell-materials interactions. Nanotechnology, 2011. 22(21).

81. Hlady, V. and J. Buijs, Protein adsorption on solid surfaces. Current Opinion in Biotechnology, 1996. 7(1): p. 72-77. 
82. Andrade, J.D., V. Hlady, and A.P. Wei, Adsorption of complex proteins at interfaces, in Pure and Applied Chemistry. 1992. p. 1777.

83. Thevenot, P., W.J. Hu, and L.P. Tang, Surface chemistry influences implant biocompatibility. Current Topics in Medicinal Chemistry, 2008. 8(4): p. 270-280.

84. Kim, D.H., et al., Microengineered Platforms for Cell Mechanobiology. Annual Review of Biomedical Engineering, 2009. 11: p. 203-233.

85. Thery, M., Micropatterning as a tool to decipher cell morphogenesis and functions. Journal of Cell Science, 2010. 123(24): p. 4201-4213.

86. MacQueen, L., Y. Sun, and C.A. Simmons, Mesenchymal stem cell mechanobiology and emerging experimental platforms. Journal of the Royal Society Interface, 2013. 10(84).

87. McBeath, R., et al., Cell shape, cytoskeletal tension, and RhoA regulate stem cell lineage commitment. Developmental Cell, 2004. 6(4): p. 483-495.

88. James, J., et al., Subcellular Curvature at the Perimeter of Micropatterned Cells Influences Lamellipodial Distribution and Cell Polarity. Cell Motility and the Cytoskeleton, 2008. 65(11): p. 841-852.

89. Thery, M., et al., Cell distribution of stress fibres in response to the geometry of the adhesive environment. Cell Motility and the Cytoskeleton, 2006. 63(6): p. 341-355.

90. Ertl, P., et al., Lab-on-a-chip technologies for stem cell analysis. Trends in Biotechnology, 2014. 32(5): p. 245-253.

91. Hart, S.R., et al., Adhesive micro-line periodicity determines guidance of axonal outgrowth. Lab on a Chip, 2013. 13(4): p. 562-569.

92. Tomba, C., et al., Tuning the adhesive geometry of neurons: length and polarity control. Soft Matter, 2014. 10(14): p. 2381-2387.

93. Kilinc, D., A. Blasiak, and G.U. Lee, Microtechnologies for studying the role of mechanics in axon growth and guidance. Frontiers in Cellular Neuroscience, 2015. 9.

94. Gupta, S.K., et al., Coordination between Extrinsic Extracellular Matrix Cues and Intrinsic Responses to Orient the Centrosome in Polarizing Cerebellar Granule Neurons. Journal of Neuroscience, 2010. 30(7): p. 2755-2766.

95. Benzina, O., et al., Morphology and Intrinsic Excitability of Regenerating Sensory and Motor Neurons Grown on a Line Micropattern. Plos One, 2014. 9(10).

96. Shi, P., K. Shen, and L.C. Kam, Local presentation of $L 1$ and $N$-cadherin in multicomponent, microscale patterns differentially direct neuron function in vitro. Developmental Neurobiology, 2007. 67(13): p. 1765-1776.

97. Oliva, A.A., et al., Patterning axonal guidance molecules using a novel strategy for microcontact printing. Neurochemical Research, 2003. 28(11): p. 1639-1648.

98. Wheeler, B.C., et al., Microcontact printing for precise control of nerve cell growth in culture. Journal of Biomechanical Engineering-Transactions of the Asme, 1999. 121(1): p. 73-78.

99. Kam, L., et al., Axonal outgrowth of hippocampal neurons on micro-scale networks of polylysine-conjugated laminin. Biomaterials, 2001. 22(10): p. 1049-1054.

100. Xu, X.H., et al., A patterned recombinant human IgM guides neurite outgrowth of CNS neurons. Scientific Reports, 2013. 3.

101. Stenger, D.A., et al., Microlithographic determination of axonal/dendritic polarity in cultured hippocampal neurons. Journal of Neuroscience Methods, 1998. 82(2): p. 167173.

102. Lodish, H.B., A.; Zipursky, S. L.; Paul, M.; David, B.; James, D, Molecular Cell Biology. 4th ed ed. 2000, New York. 
103. Ulman, A., Formation and structure of self-assembled monolayers. Chemical Reviews, 1996. 96(4): p. 1533-1554.

104. Phillips, J.E., et al., Human mesenchymal stem cell differentiation on self-assembled monolayers presenting different surface chemistries. Acta Biomaterialia, 2010. 6(1): p. 12-20.

105. Curran, J.M., R. Chen, and J.A. Hunt, The guidance of human mesenchymal stem cell differentiation in vitro by controlled modifications to the cell substrate. Biomaterials, 2006. 27(27): p. 4783-4793.

106. Valamehr, B., et al., Hydrophobic surfaces for enhanced differentiation of embryonic stem cell-derived embryoid bodies. Proceedings of the National Academy of Sciences of the United States of America, 2008. 105(38): p. 14459-14464.

107. Leyden, D.E., Silanes, surfaces, and interfaces, ed. G.a. Breach. 1986.

108. Kulangara, K. and K.W. Leong, Substrate topography shapes cell function. Soft Matter, 2009. 5(21): p. 4072-4076.

109. Das, R.K. and O.F. Zouani, A review of the effects of the cell environment physicochemical nanoarchitecture on stem cell commitment. Biomaterials, 2014. 35(20): p. 5278-5293.

110. Discher, D.E., et al., Matrix elasticity directs stem cell lineage specification. Biophysical Journal, 2007: p. 32a-32a.

111. Zouani, O.F., et al., Altered nanofeature size dictates stem cell differentiation. Journal of Cell Science, 2012. 125(5): p. 1217-1224.

112. Dalby, M.J., et al., The control of human mesenchymal cell differentiation using nanoscale symmetry and disorder. Nature Materials, 2007. 6(12): p. 997-1003.

113. Kilian, K.A., et al., Geometric cues for directing the differentiation of mesenchymal stem cells. Proceedings of the National Academy of Sciences of the United States of America, 2010. 107(11): p. 4872-4877.

114. Yim, E.K.F., S.W. Pang, and K.W. Leong, Synthetic nanostructures inducing differentiation of human mesenchymal stem cells into neuronal lineage. Experimental Cell Research, 2007. 313(9): p. 1820-1829.

115. Tay, C.Y., et al., Micropatterned matrix directs differentiation of human mesenchymal stem cells towards myocardial lineage. Experimental Cell Research, 2010. 316(7): p. 1159-1168.

116. Oh, S., et al., Stem cell fate dictated solely by altered nanotube dimension. Proceedings of the National Academy of Sciences of the United States of America, 2009. 106(7): p. 21302135.

117. Brammer, K.S., et al., Improved bone-forming functionality on diameter-controlled TiO2 nanotube surface. Acta Biomaterialia, 2009. 5(8): p. 3215-3223.

118. Heller, D.A., et al., Patterned networks of mouse hippocampal neurons on peptide-coated gold surfaces. Biomaterials, 2005. 26(8): p. 883-889.

119. Li, W., et al., NeuroArray: A Universal Interface for Patterning and Interrogating Neural Circuitry with Single Cell Resolution. Scientific Reports, 2014. 4.

120. Dowell-Mesfin, N.M., et al., Topographically modified surfaces affect orientation and growth of hippocampal neurons. Journal of Neural Engineering, 2004. 1(2): p. 78-90.

121. Esch, T., V. Lemmon, and G. Banker, Local presentation of substrate molecules directs axon specification by cultured hippocampal neurons. Journal of Neuroscience, 1999. 19(15): p. 6417-6426.

122. Fozdar, D.Y., et al., Hippocampal neurons respond uniquely to topographies of various sizes and shapes. Biofabrication, 2010. 2(3). 
123. Hoffman-Kim, D., J.A. Mitchel, and R.V. Bellamkonda, Topography, Cell Response, and Nerve Regeneration. Annual Review of Biomedical Engineering, Vol 12, 2010. 12: p. 203231.

124. Fozdar, D.Y., et al., Selective axonal growth of embryonic hippocampal neurons according to topographic features of various sizes and shapes. International Journal of Nanomedicine, 2011. 6: p. 45-57.

125. Moore, S.W. and M.P. Sheetz, Biophysics of Substrate Interaction: Influence on Neural Motility, Differentiation, and Repair. Developmental Neurobiology, 2011. 71(11): p. 1090-1101.

126. Rajnicek, A.M., S. Britland, and C.D. McCaig, Contact guidance of CNS neurites on grooved quartz: influence of groove dimensions, neuronal age and cell type. Journal of Cell Science, 1997. 110: p. 2905-2913.

127. Micholt, L., et al., Substrate Topography Determines Neuronal Polarization and Growth In vitro. PLOS ONE, 2013. 8(6): p. e66170.

128. Tonazzini, I., et al., Interaction of leech neurons with topographical gratings: comparison with rodent and human neuronal lines and primary cells. Interface Focus, 2014. 4(1).

129. Li, W., et al., Large-scale Topographical Screen for Investigation of Physical NeuralGuidance Cues. Scientific Reports, 2015. 5.

130. Hanson, J.N., et al., Textural guidance cues for controlling process outgrowth of mammalian neurons. Lab on a Chip, 2009. 9(1): p. 122-131.

131. Zhang, C., et al., Towards a human-on-chip: Culturing multiple cell types on a chip with compartmentalized microenvironments. Lab on a Chip, 2009. 9(22): p. 3185-3192.

132. Ni, X.F., et al., On-chip differentiation of human mesenchymal stem cells into adipocytes. Microelectronic Engineering, 2008. 85(5-6): p. 1330-1333.

133. Alrifaiy, A., O.A. Lindahl, and K. Ramser, Polymer-Based Microfluidic Devices for Pharmacy, Biology and Tissue Engineering. Polymers, 2012. 4(3): p. 1349-1398.

134. van Midwoud, P.M., et al., Comparison of Biocompatibility and Adsorption Properties of Different Plastics for Advanced Microfluidic Cell and Tissue Culture Models. Analytical Chemistry, 2012. 84(9): p. 3938-3944.

135. Halldorsson, S., et al., Advantages and challenges of microfluidic cell culture in polydimethylsiloxane devices. Biosensors \& Bioelectronics, 2015. 63: p. 218-231.

136. Chen, W.Q., Y.B. Sun, and J.P. Fu, Microfabricated Nanotopological Surfaces for Study of Adhesion-Dependent Cell Mechanosensitivity. Small, 2013. 9(1): p. 81-89.

137. Michel, M., et al., Deposition Mechanisms in Layer-by-Layer or Step-by-Step Deposition Methods: From Elastic and Impermeable Films to Soft Membranes with Ion Exchange Properties. ISRN Materials Science, 2012. 2012: p. 13.

138. Lee, D. and S. Yang, Surface modification of PDMS by atmospheric-pressure plasmaenhanced chemical vapor deposition and analysis of long-lasting surface hydrophilicity. Sensors and Actuators B-Chemical, 2012. 162(1): p. 425-434.

139. Norde, W. and A.C.I. Anusiem, Adsorption, Desorption and Readsorption of Proteins on Solid-Surfaces. Colloids and Surfaces, 1992. 66(1): p. 73-80.

140. Suga, T., et al., Enhanced proliferation of HeLa cells on PLLA-PCL and PLGA-PCL multiblock copolymers. Polym J, 2017.

141. Ellis, M.J. and J.B. Chaudhuri, Human bone derived cell culture on PLGA flat sheet membranes of different lactide:glycolide ratio. Biotechnol Bioeng, 2008. 101(2): p. 36977.

142. Xu, X., et al., A patterned recombinant human IgM guides neurite outgrowth of CNS neurons. Sci Rep, 2013. 3: p. 2267. 
143. Cheng, Z.A., et al., Bioactive Nanoimprint Lithography: A Study of Human Mesenchymal Stem Cell Behavior and Fate, in XIII Mediterranean Conference on Medical and Biological Engineering and Computing 2013: MEDICON 2013, 25-28 September 2013, Seville, Spain, L.M. Roa Romero, Editor. 2014, Springer International Publishing: Cham. p. 1817-1820.

144. Griffin, M.F., et al., Control of stem cell fate by engineering their micro and nanoenvironment. World Journal of Stem Cells, 2015. 7(1): p. 37-50.

145. Suzuki, M. and Y. Sakai, Effect of Protein Adsorption on Cell Attachment on Solid-Surfaces. Animal Cell Technology : Basic \& Applied Aspects, Vol 4, 1992: p. 71-76.

146. Nam, K.H., et al., Probing Mechanoregulation of Neuronal Differentiation by Plasma Lithography Patterned Elastomeric Substrates. Scientific Reports, 2014. 4.

147. Goyal, G. and Y. Nam, Neuronal micro-culture engineering by microchannel devices of cellular scale dimensions. Biomedical Engineering Letters, 2011. 1(2): p. 89-98.

148. Kim, J., et al., In vitro osteogenic differentiation of human amniotic fluid-derived stem cells on a poly(lactide-co-glycolide) (PLGA)-bladder submucosa matrix (BSM) composite scaffold for bone tissue engineering. Biomed Mater, 2013. 8(1): p. 014107.

149. Paguirigan, A.L. and D.J. Beebe, From the cellular perspective: exploring differences in the cellular baseline in macroscale and microfluidic cultures. Integr Biol (Camb), 2009. 1(2): p. 182-95.

150. Liu, L., et al., A micro-channel-well system for culture and differentiation of embryonic stem cells on different types of substrate. Biomed Microdevices, 2010. 12(3): p. 505-11.

151. Millet, L.J., et al., Microfluidic devices for culturing primary mammalian neurons at low densities. Lab on a Chip, 2007. 7(8): p. 987-994. 


\section{Chapter. 3 Materials and methods}

\subsection{Substrate preparation, modification and microfabrication}

In the below section, the techniques used to induce surface functional groups for ECM protein immobilization as well as microfabrication of topographic features on PDMS substrates is explained.

\subsubsection{PDMS preparation and surface chemical modification}

PDMS substrates were prepared by mixing 10 parts of silicone elastomer base to 1 part of curing agent (SYLGARD, Dow Corning, USA) followed by casting onto a flat polystyrene dish or wellplate. The PDMS was then degassed in a vacuum oven for 30 min to remove all the air bubbles and cured at $70{ }^{\circ} \mathrm{C}$ for $90 \mathrm{~min}$. The cured PDMS substrates were split into three groups, and their surfaces were treated as follows.

Silanes varying in structure and functionality (APTES, APTMS and MPTMS from Sigma Aldrich, Singapore) were used as per the experimental requirements. Depending upon the silanization experiment PDMS substrate groups (Silane + protein or Silane + GA + protein) were subjected to oxygen plasma for $3 \mathrm{~min}$ in the plasma cleaner (Harrick Plasma-PDC 32G) followed by immersing the PDMS substrates in $10 \%$ (volume ratio in DI water) silane (Sigma-Aldrich, Singapore) at $50{ }^{\circ} \mathrm{C}$ for $2 \mathrm{~h}$. The silane solution was removed, and the samples were washed twice in nuclease free water. The (Silane + GA) substrate group was further immersed in a $2.5 \%$ GA (SigmaAldrich, Singapore) solution at room temperature for $1 \mathrm{~h}$. GA solution was then removed, and the samples were washed twice in nuclease-free water. The substrates in which the direct effect of functional group of the silane on the cells was examined were not treated with GA. All three groups (protein (Flat PDMS), Silane + protein, and Silane + GA + protein) were then immersed in either $0.1 \mathrm{mg} / \mathrm{mL}$ of a fibronectin (FN) (Life Technologies, Singapore), collagen type 
1 (Col1) solution (Life Technologies, Singapore), Laminin (LN) (Life Technologies, Singapore) and poly-l-lyisne (PLL) (Life Technologies, Singapore) and stored at $4{ }^{\circ} \mathrm{C}$ overnight. Finally, the protein solution was removed, and the samples were washed twice with nuclease-free water. The PDMS substrates were sterilized under UV light for $60 \mathrm{~min}$ prior to cell culture experiments. Tissue culture plates (TCP) (Nunc, Singapore) or native (plain/unmodified) PDMS were used as controls in the experiments depending upon the surface chemical/topographical phenomena under investigation.

\subsubsection{Fabrication of topographic micro-features by Photo-lithography}

Depending upon the size, detail, aspect-ratio and complexity of the micro-features to be finally built on the PDMS surfaces, positive/negative photolithography was implemented [1]. SU 8 negative photoresist is often utilized in microfabrication techniques. Upon ultra violet (UV) exposure, SU 8 turns insoluble. After development, UV exposed portions remain on the substrate.

Figure 3-1 depicts the difference between a negative and positive photoresist.

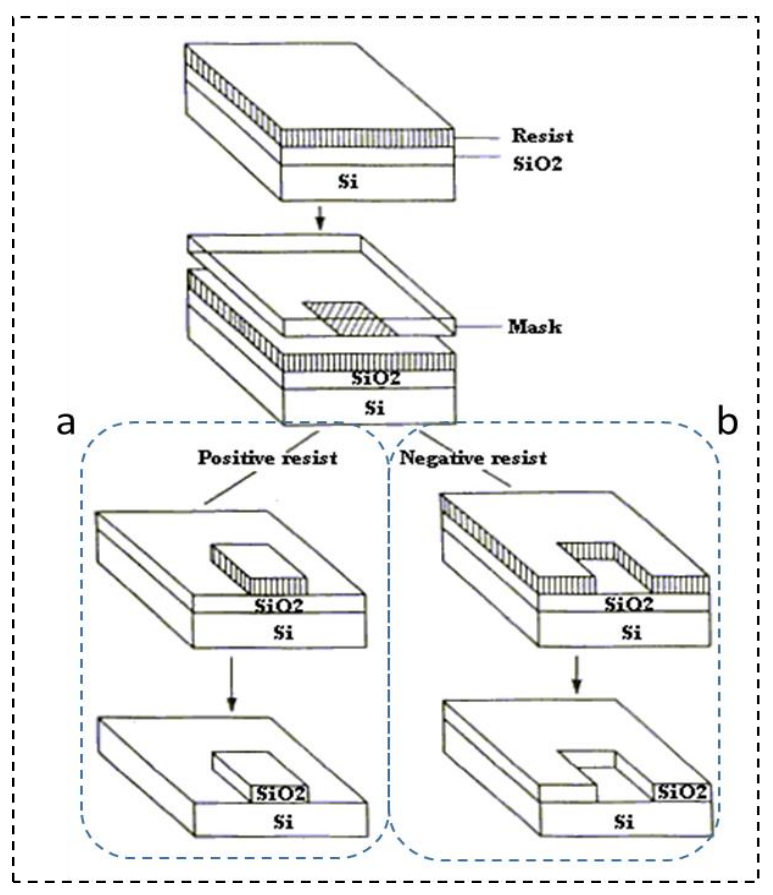

Figure 3-1: Schematic representation of differences between (a) positive and (b) negative photolithography. 
In our work, we used the positive photo resist (route a) to fabricate topographical features for the neuronal substrates and negative photo resist (route b) to fabricate hMSC topographic substrates. Although SU8-3010 and SU8-25 (Microchem, US) based features were initially developed for neuronal substrates, undesired resolution and the feature quality was encountered especially under $5 \mu \mathrm{m}$ sizes. We also observed the formation of thicker features with randomly silhouetted boundaries. Since a low feature size of upto $2 \mu \mathrm{m}$ was desired in the neuronal substrates and arrays of multiple shapes varying in geometry arrays was desired in the fabrication, the positive photoresist AZ1512 (Microchem, US) was finally chosen to form stable and fine microstructures. In contrast, since higher features sizes of $\leq 10 \mu \mathrm{m}$ was desired for the fabrication of topographical features for the hMSC studies, we chose to fabricate these substrates by layer-by-layer spin coating and exposure on the SU8-3050 (Microchem, US) negative photoresist.

\subsubsection{Fabrication of Hole-pillar and groove micro-features for hMSC cell studies}
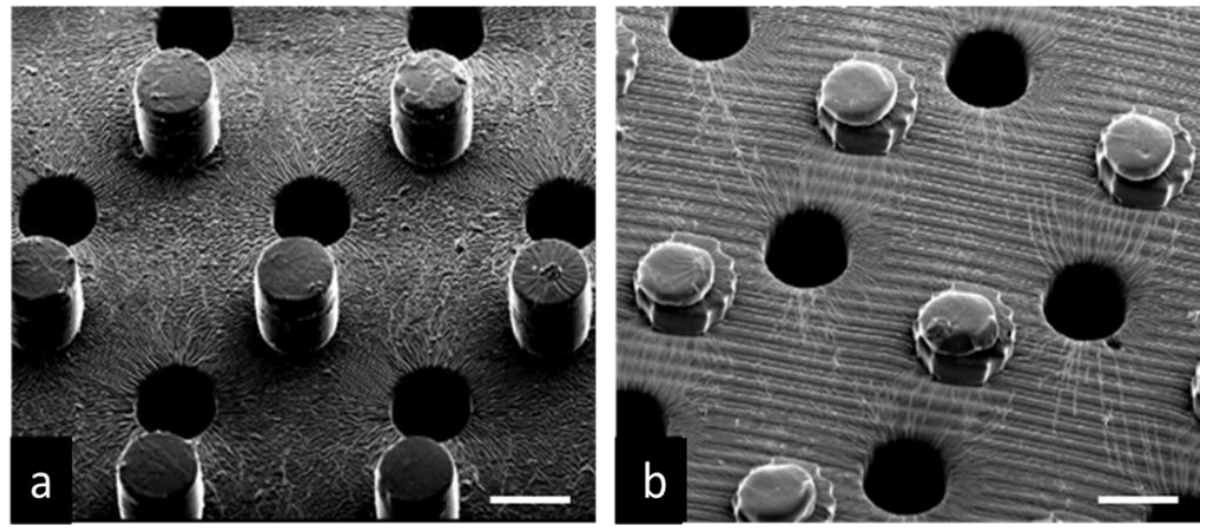

Figure 3-2: SEM micrograph of the (a) hole-pillar and (b) ridged pillar-hole-groove topographical micro-features patterned on PDMS substrate. Scale bar: $100 \mu \mathrm{m}$

PDMS hybrid patterns were generated by replication from a silicon mold with two SU-8 layers of micro-patterns (Figure 3-2). The first layer contained features of the $\Phi 100 \mu \mathrm{m}$ through-holes. The second layer of SU-8 contained features of the $\Phi 100 \mu \mathrm{m}$ pillars array. The two-layer micro- 
patterns were fabricated sequentially with alignment markers by following the standard photolithography process.

First, a layer of SU8-3050 photoresist (MicroChem, US) was spin-coated on the silicon wafer at a speed of $1000 \mathrm{rpm}$ for 30s to reach the thickness of approximately $100 \mu \mathrm{m}$. After baking on a hot plate at $95{ }^{\circ} \mathrm{C}$ for $45 \mathrm{~min}$, it was exposed under $365 \mathrm{~nm} \mathrm{UV}\left(8 \mathrm{~mW} / \mathrm{cm}^{2}\right)$ for $30 \mathrm{~s}$. Then the silicon wafer was baked for photoresist further crosslinking at $65^{\circ} \mathrm{C}$ for $1 \mathrm{~min}$ and $95{ }^{\circ} \mathrm{C}$ for $5 \mathrm{~min}$. When it reached to room temperature, it was immerged in SU8 developer for $20 \mathrm{~min}$ and rinsed with isopropanol (IPA), resulting in an SU8 defined holes (first layer) or pillars (second layer) array. For the holes-grooves-pillars hybrid patterns, another layer of SU8 was added between these two layers with alignment to generate microgrooves. SU-8 3050 was spin-coated on the silicon wafer with the first layer of through-hole patterns at the speed of $4000 \mathrm{rpm}$ for $30 \mathrm{~s}$. The thickness of this layer was around $35 \mu \mathrm{m}$. The wafer was baked at $95{ }^{\circ} \mathrm{C}$ for $15 \mathrm{~min}$ and exposed under UV for 25 s. Post exposure bake was directly performed after exposure at $65{ }^{\circ} \mathrm{C}$ for 1 min and $95{ }^{\circ} \mathrm{C}$ for 5 min and finally developed for $10 \mathrm{~min}$.

PDMS pre-polymer was prepared by mixing silicon elastomer base and curing agent at 10:1 ratio and degassed under vacuum to remove air bubbles. The silicon mold was treated with chlorotrimethylsilane (Sigma Aldrich, Singapore) for $3 \mathrm{~min}$, and then the PDMS pre-polymer was poured onto the silicon mold to uniformly cover all the patterned areas and degassed again to remove the air bubbles generated during the pouring procedure. To get a through-hole PDMS membrane, the silicon mold was spin-coated at $1000 \mathrm{rpm}$ for $30 \mathrm{~s}$. After baking on an $80{ }^{\circ} \mathrm{C}$ hotplate for $15 \mathrm{~min}$, the cured PDMS membrane with hybrid micropatterns could be gently peeled off from the silicon mold. 


\subsubsection{Fabrication of geometrical micro-trap shape array for neuronal cell studies}

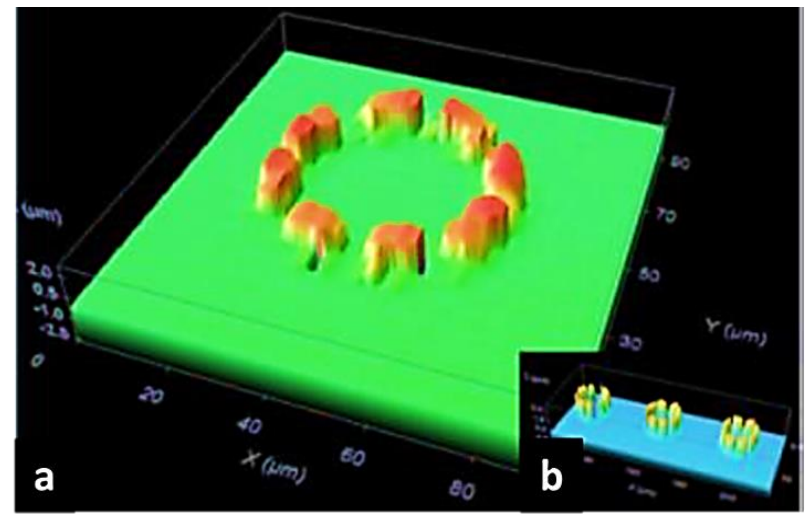

Figure 3-3: (a) Confocal profile micrograph of circular micro-trap shape micro-feature patterned on PDMS (b) (inset) circular micro trap array with equidistant features

The micro scale features were developed on the silicon wafer by using a positive photoresist AZ1512. Briefly, the silicon wafer was rinsed with acetone followed by immersion in isopropanol and blown dry with nitrogen gas. The wafer was spin coated with AZ1512 (Micro-chemicals $\mathrm{GmbH}$, Germany) photoresist at $1000 \mathrm{rpm}$ and prebaked for 45 seconds at $110^{\circ} \mathrm{C}$. The coated wafer was secured tightly to the glass photomask by clamping and UV exposed for 30 seconds under $13 \mathrm{~mJ} / \mathrm{cm} 2$ energy conditions. Subsequently, the wafer was quickly rinsed in AZ developer solution (1:2) for 3 mins to yield a completed template master wafer.

PDMS mixture was prepared by mixing Sylgard 184 base to curing agent (Dow Corning Co., USA) in the ratio 10:1 by constant agitation and left to degas completely within a vacuum oven for 30 mins. The mixture was then poured evenly over silicon wafer and cured at $70 \pm 3^{\circ} \mathrm{C}$ for two hours. After curing, the PDMS was slowly peeled off from the master wafer. Confocal profiling of the negative features on the master wafer and the PDMS micro-pillars confirmed the height of the features to a range between 3.5-3.8 $\mu \mathrm{m}$. The pillar and the micro-gap width on the PDMS substrates were measured as $5 \mu \mathrm{m}$ and $3 \mu \mathrm{m}$ respectively. Prior to cell culture, the PDMS substrates were rinsed in $70 \%$ ethanol, dried in $\mathrm{N}_{2}$ gas and subjected to oxygen plasma for 2.5 min in the plasma cleaner (Harrick Plasma-PDC 32G) followed by immediate immersion in poly-1- 
lysine solution (Sigma Aldrich, Singapore) in which the substrates were stored overnight at $4^{\circ} \mathrm{C}$. In the following day, before cell culture, the substrates were washed thoroughly in 1x PBS (Life Technologies, Singapore) and sterilized under UV for 30-40 mins.

\subsubsection{Fabrication and modification of PDMS microchannels for hMSC/neuron study}

The photomask for chip fabrication was designed with AUTOCAD 2014 and printed on highresolution transparency films (CAD/Art Services Inc., Oregon, US). For the fabrication of PDMS microchannels for hMSC studies, SU8-2050 photoresist was spin-coated at $1500 \mathrm{rpm}$ to create an evenly spread film, soft baked to evaporate the solvent and densified before being covered by the photomask and exposed to highly collimated UV light. After UV exposure, the SU-8 was further subjected to post exposure baking to selectively cross-link the exposed portion of the photoresist. The non-exposed portion of the film was etched away by immersing in a developer solution.

For PDMS micro channels devised for neuronal studies, separate photomask with compartmental microfluidic design was used. Initially a thin layer of SU-8 (Microchem®, Singapore) was spin coated on the silicon wafer to a depth of $30 \mu \mathrm{m}$. Patterning of the microfluidic design was performed by similar photolithography. A biopsy punch (Miltex®, Singapore) of $4 \mathrm{~mm}$ inner diameter was used to produce the inlet and outlet reservoirs of the micro-device. Devices were then assembled by plasma bonding to a thin coated planar PDMS layer supported by a glass coverslip (thickness $\sim 170 \mu \mathrm{m}$ ) at the bottom.

PDMS microchips were fabricated by replication of microchannel features by pouring PDMS (10:1 base and curing agent ratio) on the SU-8 masters, curing for $2 \mathrm{~h}$ at $70^{\circ} \mathrm{C}$ and finally peeling off of the PDMS structure (Schematic shown in Figure 2-20 b). The PDMS microfluidic chip was plasma-treated prior to bonding on flat PDMS substrates. Immediately following the plasma bonding, the surface modification of micro-channels was initiated by silane (APTES/APTMS/MPTMS) and protein treatment in the similar conditions as described in 
modification of planar substrates (Section 3.1.1). Chemical treatment and washing steps were performed by maintaining optimal hydrodynamic flows of the reagents/washing buffers at the inlet and exit reservoir ports of the microchip.

\subsection{Characterization of substrate chemical and topographical}

\section{properties}

To characterize biophysical and biochemical modification of the substrates, it is essential to obtain key molecular, nano-scale as well as micron scale changes induced by respective modification. The below section deals with the details of these techniques and the analysis of various surface characteristics of interest in the cell-material interactions.

\subsubsection{Characterization of Surface's Hydrophilic Properties}

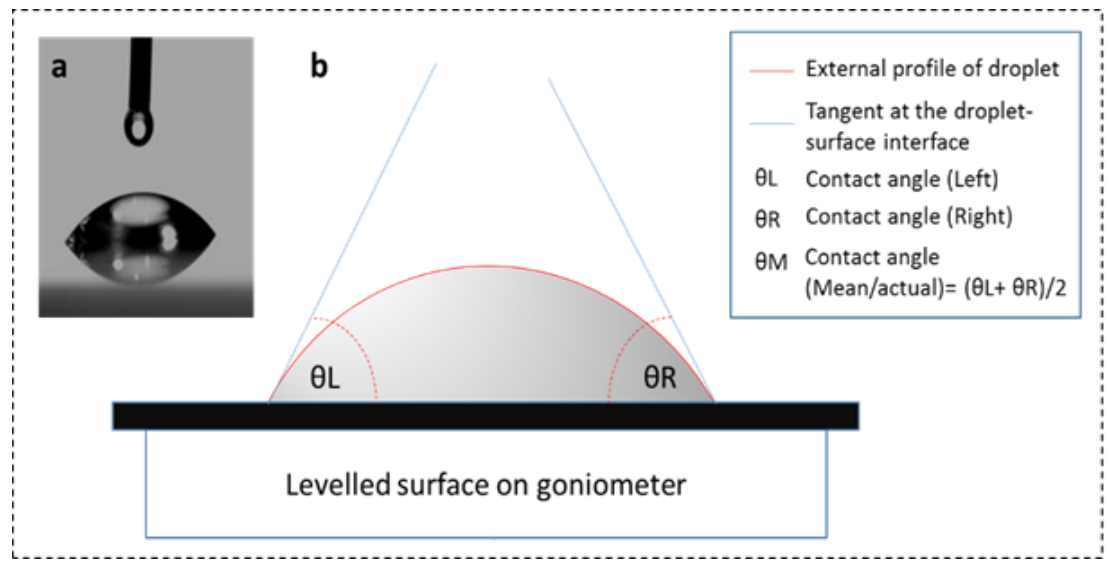

Figure 3-4: (a) Dispensing 5ul droplet on the surface from the vertically mounted syringe (b) Measurement of water contact angle of the static/sessile droplet

Water contact-angle measurements were determined with a theta optical tensiometer (Attension, Finland, Europe) (Figure 3-4 a). Briefly, $5 \mu$ L droplets of Milli-Q water (resistivity >10 MO) was brought into contact with the surface of the PDMS substrates, and the contact angles were 
measured with the static sessile drop tangent method [2] using the Drop Shape Analysis software (Attension ${ }^{\circledR}$ ), where the angle formed between the substrate surface and the tangent to the drop surface was determined (Figure 3-4 b). At least three points of contact were measured for each sample.

\subsubsection{Surface roughness measurements}

Surface roughness of the different PDMS substrates were determined by an atomic force microscopy (AFM, Asylum Research, Model MFP-3D) with the use of a tapping mode AFM probe comprising of a silicon tip with radius of $28 \pm 10 \mathrm{~nm}$ and spring constant range of 0.5 $4.4 \mathrm{~N} / \mathrm{m}$ (Asylum Research). $20 \mu \mathrm{m}$ x $20 \mu \mathrm{m}$ topographical images were scanned at $0.8 \mathrm{~Hz}$, a set point of $0.7 \mathrm{~V}$ and a resolution of 256 pixel resolutions. At least 3 points of contacts were analyzed for each PDMS surface.

\subsubsection{Determination of elemental composition (XPS)}

X-ray photoelectron spectroscopy (XPS) was performed to analyze the elemental composition of the samples. XPS spectra were taken in normal emission at 9 to 10 mbar within 10 min. All C $1 \mathrm{~s}$ peaks corresponding to hydrocarbons were calibrated to a binding energy of $284.8 \mathrm{eV}$ to correct for the energy shift caused by charging. The spectra were analyzed using the Casa XPS software. A $50 \mathrm{eV}$ pass energy using empirical Wagner sensitivity factors was set to obtain the required region scans on the surface.

\subsubsection{Surface Protein Quantification}

A micro-BCA Protein Assay Kit (Thermo Scientific, Singapore) was used to determine the amount of protein that was retained on different PDMS substrates. Following the overnight incubation in the $0.1 \mathrm{mg} / \mathrm{mL}$ protein solution, the protein solution was discarded. The PDMS surfaces were the incubated with $0.05 \%$ Tween 20 (Sigma-Aldrich, Singapore) for 30 min on a shaker followed by washing twice with nuclease-free water to remove loosely bound or non- 
adherent protein molecules. The adhered protein on the PDMS surfaces was then determined as per the stated protocol in the Micro-BCA Protein Assay Kit, where the absorbance of specimens was measured at $562 \mathrm{~nm}$ with Multiskan Spectrum microplate reader (Thermo Scientific, Singapore). The results were then expressed as a percentage ratio of the surface-bound protein concentration to the initial protein seeding concentration to reflect the percentage of protein retention on the PDMS surfaces. For the protein quantification shown in Chapter 4.2, the data is obtained from three separate data sets for day 1, day 7 and day 14. All three sets contain four replicates each of plain PDMS, APTES treated PDMS and APTES+GA treated PDMS on with Col1 coating was performed. Then samples were stored in 1x PBS under incubation conditions $\left(37^{\circ} \mathrm{C}\right.$ and $\left.5 \% \mathrm{CO}_{2}\right)$ until measurement. During each assay, the PDMS surfaces were incubated with 0.05 vol\% Tween 20 for 30 min on a shaker followed by washing twice with nuclease-free water to remove loosely bound/non-adherent protein molecules.

\subsubsection{Surface profile analysis by profiler and SEM}

The micro scale topographical modification of PDMS substrates were assessed from both confocal profiling as well as SEM imaging. The confocal profiling of microfabricated PDMS could reveal any micron range inconsistencies which could have been encountered either during Si wafer development or post curing or PDMS mechanical peeling off procedures. Subsequently SEM was performed for more detailed investigation of microfeatures and fine finishing of the features. Hence both the techniques were used during the optimization of micro-fabrication. 


\subsection{Cell culture and cell fate analysis}

\subsection{1 hMSC isolation, culture and differentiation}

Human mesenchymal stem cells (hMSCs) were harvested from human bone marrow aspirates under institutional guidelines and routinely cultured. Briefly, bone marrow was aspirated from the porcine iliac crest under aseptic conditions and washed with $1 \times$ PBS (First Base, Singapore). The bone marrow stromal cells were then re-suspended in Dulbecco's modified Eagle's medium (DMEM) (Life Technologies, Singapore) supplemented with 10\% fetal bovine serum (FBS) (Life Technologies, Singapore), a penicillin $(100 \mu \mathrm{g} / \mathrm{ml}) /$ streptomycin $(100 \mu \mathrm{g} / \mathrm{mL})$ mixture (Life Technologies, Singapore), and $2 \mathrm{mM}$ Glutamax (Life Technologies, Singapore) before seeding into a culture flask for culture in a humidified atmosphere of $95 \%$ air and $5 \% \mathrm{CO}_{2}$. Non-adherent cells were removed by washing after $72 \mathrm{~h}$, and the adhered mesenchymal stem cells were further expanded upon reaching confluence. This protocol was established to obtain hMSCs that demonstrated good purity and stability while maintaining the original cellular morphology and differentiation capability in their earlier passages. hMSCs of passages 2-4 were used in the experiments for this study.

For the differentiation of hMSCs, 6000 cells $/ \mathrm{cm}^{2}$ and $3 \times 10^{4}$ cells $/ \mathrm{cm}^{2}$ were allowed to adhere on the substrate surfaces prior to osteogenic and fibroblastic differentiation respectively. Induction of osteogenic differentiation in hMSCs was initiated by the introduction of osteogenic medium comprising of low glucose DMEM supplemented with $10 \% \mathrm{FBS}, 50 \mu \mathrm{g} / \mathrm{ml}$ ascorbic acid, $1 \mathrm{mM}$

Sodium Pyruvate, $100 \mu \mathrm{g} / 100 \mu \mathrm{g} \quad$ Penicillin/Streptomycin, $1 \mathrm{X}$ Glutamax, 10mM $\beta$ Glycerophosphate, $10 \mathrm{mM}$ dexamethasone. On the other hand, fibroblastic differentiation was induced with low glucose DMEM supplemented with $50 \mu \mathrm{g} / \mathrm{ml}$ of ascorbic acid. The cells were then cultured in a humidified atmosphere of $95 \%$ air and $5 \% \mathrm{CO}_{2}$, with their differentiation medium changed every 2-3 days. At least 4 specimens were collected for each individual analysis. 


\subsubsection{Characterization of hMSC cell behaviors}

Morphological and quantitative evaluation of hMSCs were performed by several techniques which are explained in the below section. A primary motive is to consider both qualitative and quantitative assessment is to correlate cell morphological changes induced by the underlying substrate chemistry or topography with their overall adhesion, proliferation and differentiation behaviors.

\subsubsection{Morphological analysis of hMSCs}
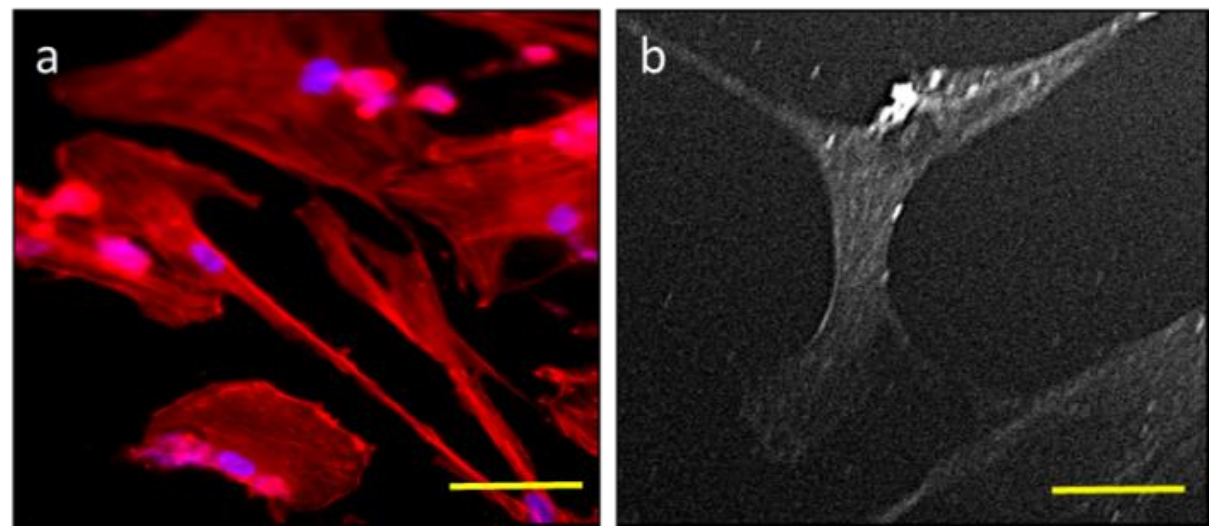

Figure 3-5: Morphological analysis of hMSCs by (a) F-actin+DAPI cytoskeleton and nucleus staining (b) SEM profiles of cells attached on flat substrate

After 3 days of cell culture, hMSCs attached on different substrates were fixed with $10 \%$ formalin (Sigma-Aldrich, Singapore), permeabilized with 0.1\% Triton X-100 (Sigma-Aldrich, Singapore), blocked with 1\% BSA (Sigma-Aldrich, Singapore), and incubated with Rhodamine-conjugated Phalloidin (Millipore, Singapore) for $60 \mathrm{~min}$ and 4',6-diamidino-2-phenylindole (DAPI, Millipore, Singapore) for $5 \mathrm{~min}$. The localization and organization of F-actin were observed under an IX71 inverted fluorescence microscope (Olympus, Singapore) (Figure 3-5a). Further detailed morphology of hMSCs cell pseudopods and anchorage sites on the substrates were obtained by 
SEM imaging (Figure 3-5b). For this, the cell laden substrates were subjected to serial drying steps by dipping the substrates in $100 \%$ ethanol: water mixture of $50 \%, 60 \%, 70 \%, 80 \%, 90 \%$ and absolute concentration for 10 min per step. The dried specimens were sputter-coated with a thin layer of gold. Finally, the hMSC cell body integration with PDMS microstructures was visualized with a SEM microscope (JSM6390LA, JEOL Asia, Singapore).

\subsubsection{Cell Proliferation Assay}

PrestoBlue cell viability reagent (Life Technologies, Singapore) was used to assay the proliferation activity if hMSCs on different modified PDMS surfaces for 2 weeks. Briefly, hMSCs were seeded at a density of $1.5 \times 10^{3}$ cells per well $(1.9 \mathrm{~cm})$ and incubated in a humidified atmosphere of $5 \% \mathrm{CO}_{2}$ at $37{ }^{\circ} \mathrm{C}$. The medium was changed twice per week. On third, seventh, and 14th days, the culture medium was removed, and the cells were washed twice with 1x PBS before they were incubated with DMEM containing $10 \%$ PrestoBlue reagent for $1 \mathrm{~h}$ at $37^{\circ} \mathrm{C}$ in a humidified, $5 \% \mathrm{CO}_{2}$ atmosphere. DMEM containing 10\% PrestoBlue reagent was incubated in the wells with no cells and served as the blank control. The absorbance of the reduced PrestoBlue reagent was read at $570 \mathrm{~nm}$ (excitation) and $600 \mathrm{~nm}$ (emission) with a Multiskan Spectrum microplate reader (Thermo Scientific, Singapore). Because the number of viable cells correlated with the reduction level of the PrestoBlue dye, the absorbance readings were converted and expressed as the percentage reduction of the PrestoBlue reagent according to the manufacturer's protocol.

\subsubsection{Characterization of Cell Adhesion}

For the analysis of cell adhesion capability on different modified PDMS surfaces. hMSCs were seeded at a density of $1.0 \times 10^{4}$ cells per well $\left(1.9 \mathrm{~cm}^{2}\right)$ and incubated in a humidified atmosphere of $95 \%$ air and $5 \% \mathrm{CO}_{2}$ for 90 min. The wells were then rinsed twice with $1 \mathrm{x}$ PBS to remove the non-adherent cells, and the adherent cells were frozen at $-80{ }^{\circ} \mathrm{C}$. The frozen cells were then 
thawed and lysed by the addition of a cell lysis buffer containing 1× CyQUANT GR Dye from the CyQUANT Cell Proliferation Assay Kit (Life Technologies, Singapore) for 5 min. Fluorescence was then measured directly with an Infinite M200 series plate reader (Tecan Asia, Singapore) with excitation at $485 \mathrm{~nm}$ and emission detection at $535 \mathrm{~nm}$. The readings were then expressed as a fold difference relative to the fluorescence intensity of the TCP (control). Because the DNA content is constant within the cell, this method uses the DNA content (reflected by fluorescence intensity) that was obtained by cell lysis to correlate to the number of cells that remained adhered on the surface. This method has proved to be a sensitive, accurate, and simple deterministic means for correlating DNA content to cell density and thus provide an accurate quantification of the cell adhesion under conditions of different surface chemistries.

For hMSCs, the cell adhesion area was determined as follows. hMSCs were seeded onto 6-well plates at a seeding density of $1.0 \times 10^{4}$ per well $(9.6 \mathrm{~cm})$ and incubated in a humidified atmosphere of $95 \%$ air and $5 \% \mathrm{CO}_{2}$ for $4 \mathrm{~h}$. The adhered cells were then fixed with $10 \%$ formalin (Sigma Aldrich, Singapore) overnight, permeabilized with 0.1\% Triton X-100 (Sigma Aldrich, Singapore), blocked with 1\% BSA (Sigma-Aldrich, Singapore), and incubated with TRITCconjugated Phalloidin (Millipore, Singapore) for $60 \mathrm{~min}$ and DAPI (Millipore, Singapore) for 5 min. The images were viewed under an IX71 inverted fluorescence microscope (Olympus, Singapore), and the area of individual cell adherence was analyzed by Image-Pro Plus (Media Cybernetics, Rockville, MD).

\subsubsection{Histological staining and bio-mineralization analysis}

Osteogenic cells (day 21) and fibroblastic cells (day 14) were washed once with 1X PBS before fixing with $10 \%$ formalin. Next the cells were washed with 1X PBS before histological staining was performed. To assess the osteogenic differentiation of hMSCs on different modified surfaces, cells were immersed in alizarin red solution for 5 min followed by gentle washing with distilled water until non-specific staining was removed. The presence of brick-red nodules revealed the 
presence of mineralization deposition during osteogenic differentiation. On the other hand, fibroblastic differentiation of hMSCs on different modified surfaces can be assessed by PicroSirus red stain. Briefly, the cells were immersed in Sircol Dye Reagent (Biocolor, County Antrim, United Kingdom) and washed twice with $0.05 \%$ glacial acetic acid (Merck Millipore, Singapore). Alizarin red stained images were taken with a Canon 50D DSLR Camera (Canon, Singapore) while Picro Sirius red stained images were taken with Olympus IX71 inverted microscope equipped with polarizing lens (Olympus, Singapore).

\subsubsection{Gene Expression Analysis}

Fibroblastic and osteogenic cells were collected after 2 weeks, and the total RNA was extracted with RNeasy Kit (Qiagen, Singapore), and its concentration was quantified with Nanodrop ND2000 (Thermo Scientific, Delaware, USA). 100ng of total RNA was then reverse-transcribed to cDNA with the use of ScriptTM cDNA synthesis kit (Bio-rad Laboratories, Singapore). A realtime PCR assay using SYBR PCR Master Mix Kit (Life Technologies, Singapore) and StepOnePlusTM Real Time PCR Systems (Life Technologies, Singapore) was used to measure the mRNA expression level of fibroblastic or osteogenic differentiation associated gene markers. Primers for quantifying gene expression includes collagen type 1 (Col1), osteocalcin (OCN) and alkaline phosphatase (ALP) for osteogenic differentiated cells, and collagen type 1 (Col1) in fibroblastic differentiation are listed in the respective publications. Real-time PCRs were initiated at $95^{\circ} \mathrm{C}$ for $10 \mathrm{~min}$, followed by 40 -cycle amplification comprising of a denaturation step at $95^{\circ} \mathrm{C}$ for 15 seconds, and an extension step at $60{ }^{\circ} \mathrm{C}$ for $1 \mathrm{~min}$. All data were normalized to the GAPDH mRNA level, and later expressed as mRNA relative change with reference to the hMSCs prior to differentiation using the Livak method. 


\subsubsection{Isolation and culture of nascent cortical neurons}

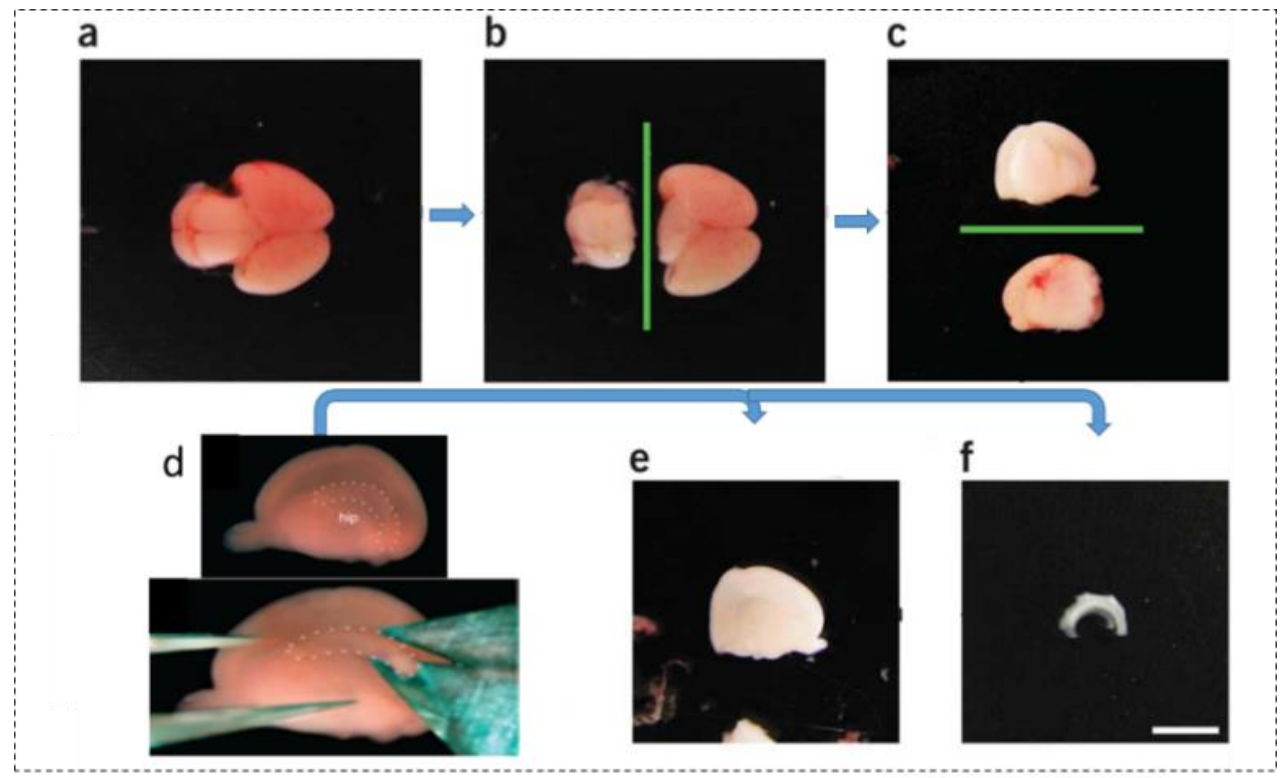

Figure 3-6: Dissection steps involved in the isolation of cortical hemisphere from the E18/P1 rat brain. (a) Isolated brain (b) Separation of cerebellum (c) Separation of corical hemispheres (d-f) Separation of hippocampus and the cortex tissues.

Cortical hemispheres from the frontal brain region of embryonic day 18 (E18) Sprague Dawley® rat pups (Invivos ${ }^{\circledR}$, Singapore) were carefully dissected, by sequential tissue separation (Figure 3-6). The animal works performed in this study were approved by the Institutional Animal Care and Use Committee (IACUC) at Nanyang Technological University, Singapore and abided by the guidelines for ethical treatment of animal specimens. Later, the tissues were gently dissociated by trituration after digesting for $15 \mathrm{~min}$ in a papain (Life Technologies, Singapore) based digestion buffer $(20 \mu \mathrm{g} / \mathrm{ml})$ within a $37^{\circ} \mathrm{C}$ water bath at neutral $\mathrm{pH}$ and allowed to digest until the formation of a smooth homogenous precipitate. The enzyme based buffer suspension was removed after centrifugation and the cells were re-suspended in a fresh medium consisting of $50 \%$ minimal essential medium (MEM) (Gibco®, Singapore) supplemented with $10 \%$ fetal bovine serum (Life Technologies, Singapore) and 50\% NB medium (Neurobasal ${ }^{\mathrm{TM}}$ with B27 supplement and $0.5 \mathrm{mM}$ glutamine, Life Technologies, Singapore) containing $25 \mu \mathrm{M}$ glutamate (Life Technologies, 
Singapore) and $25 \mu \mathrm{M} \beta$-mercaptoethanol (Sigma Aldrich, Singapore). The cells were then seeded onto the modified PDMS micro-fabricated substrates at a concentration of $0.25 \times 10^{6}$ cells $/ \mathrm{ml}$ and the initial medium change was performed within 4 hours. Subsequent medium changes were performed on alternate days.

\subsubsection{Characterization of neuron cell behaviors}

\subsubsection{Immunostaining with neuronal and glial cell markers.}

The substrates were rinsed with PBS (1x) and fixed in 3\% glutaraldehyde solution for $20 \mathrm{~min}$ at room temperature. Cells were permeabilized for $15 \mathrm{~min}$ in $0.1 \%$ Triton X-100 (Sigma-Aldrich, Singapore) solution and treated for 30 min with $1 \%$ bovine serum albumin (BSA) (Life Technologies, Singapore) in PBS to block any nonspecific binding of the antibodies. Then, the cells were incubated with neuron-specific Microtubule Associated Protein-2 (MAP2) (Millipore, Singapore) or B-tubulin (Millipore, Singapore) and glial specific Glial Fibrillary Acidic protein (GFAP) (Millipore, Singapore) in 1:1000 dilution and stored overnight at $4^{\circ} \mathrm{C}$. On the following day, the cells were incubated with Alexafluor-488 and Alexafluor-555 secondary antibodies (Life Technologies, Singapore) against the neuronal and glial primary antibodies and stored overnight at $4^{\circ} \mathrm{C}$. Finally, the cells were also stained with nuclear DAPI (Life Technologies, Singapore) dye for $5 \min$.

\subsubsection{Neurite Analysis on chemically modified flat substrates}

Neurite analysis was performed using the ImagePro plus image analysis tool (Media Cybernetics Inc. Rockville, USA). Neurons were imaged in a bright-field microscope at $10 \times$ magnification on images collected on the 3rd, 7th, 13th, and 21st days in vitro. After image collection, image contrast was adjusted on the software to threshold coloration and contrast for accurate analysis of 
neurite details such as primary branching and neurite length. These data were exported to MS Excel for tabulation and analysis.

\subsubsection{Neurite tracing on micro-patterned substrates}

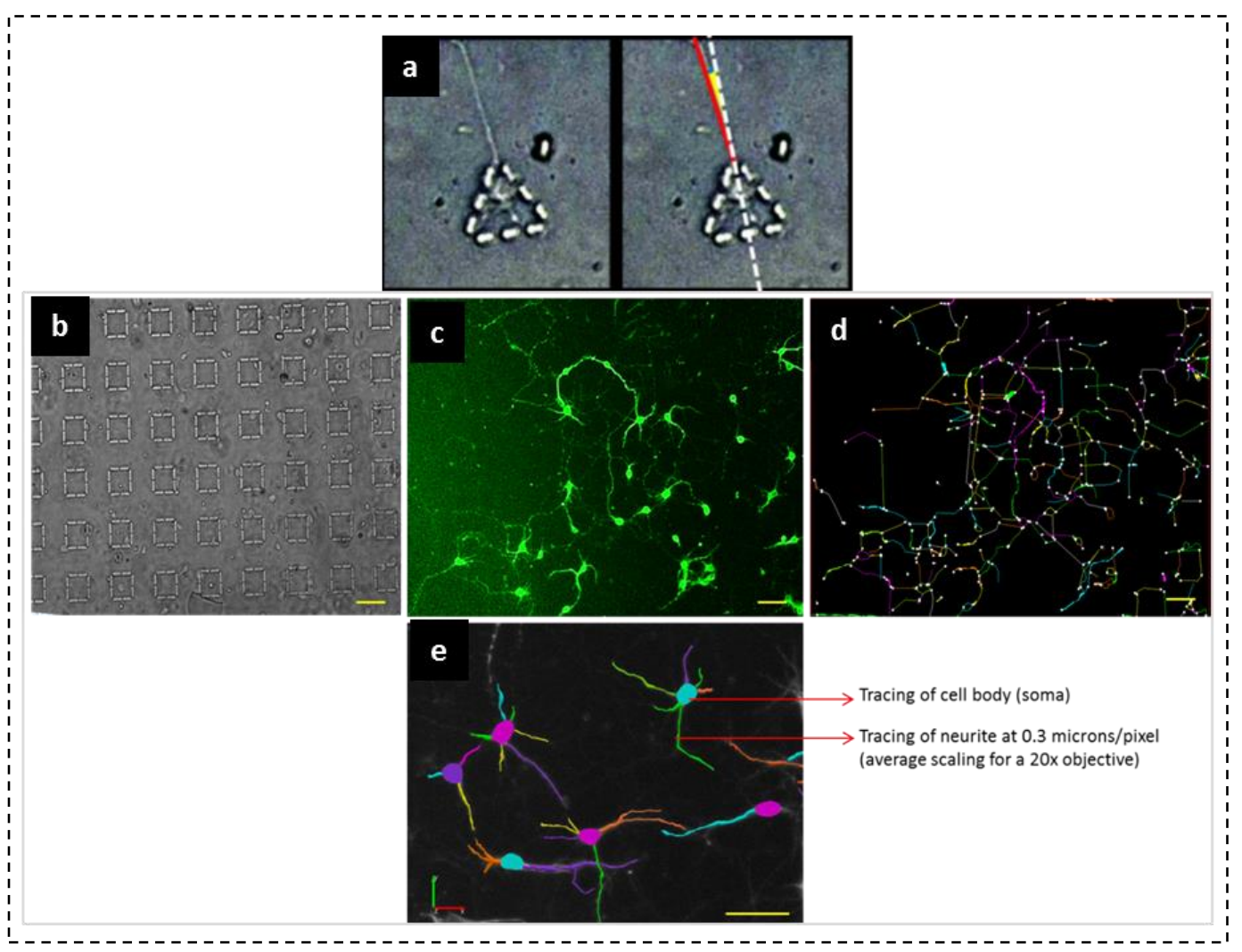

Figure 3-7: (a) Neurite angle calculation (b) Brightfield image (c) Fluorescent image of B-III tubulin immunostaining (d) Resultant neurite tracing performed on the fluorescent image in Neurolucida 360 and later exported in Neurolucida explorer for quantitative analysis (e) Enlarged illustration of neurite tracing performed on Neurolucida 360.

Neurite deflection analysis on single neurons at neurites exited from the micro-gaps along the curved and angular features on each geometrical feature were performed by tracing in NeuronJ (ImageJ plugin) and plotted in MatLab (MathWorks ${ }^{\circledR}$, Inc., USA). Neurite preferential alignments in networks were performed on the traces of fluorescent immunostaining images and the resultant polar plots were obtained using Neurolucida 360 and Neurolucida Explorer (MBF Bioscience®, VT, United States) respectively (Figure 3-7). To ensure consistency of image analysis, images 
were captured at the same magnification and exposure. The polar histogram representing the percentage of the total process length of the neurons in the entire network which fall under specific angle ranges (Neurolucida Explorer) and the average neurite length in networks were obtained from at least five network trace datasets.

\subsection{Statistical Analysis}

The paired Student's t-test was adopted for determining the statistical significance between groups using Minitab 16 Statistical Software (Minitab Inc., State College, PA). All experiments were performed in quadruplicate. A p value of $<0.05$ was deemed to be statistically significant (with $95 \%$ confidence).

\subsubsection{References}

[1] Madou, M.J., Fundamentals of Microfabrication and Nanotechnology. Vol. 1. 2011.

[2] Yuan, Y. and T.R. Lee, Contact Angle and Wetting Properties, in Surface Science Techniques, G. Bracco and B. Holst, Editors. 2013, Springer Berlin Heidelberg: Berlin, Heidelberg. p. 3-34. 


\section{Chapter. 4 Effects of surface chemical modification in regulating hMSC behaviors and potency}

\subsection{Enhanced adhesion and proliferation of hMSCs on covalently crosslinked ECM protein functionalized surfaces}

\subsubsection{Background}

For the last decade, poly (dimethyl siloxane) (PDMS) has received much attention as a biomaterial in the fabrication of biochips for multiple biological applications including the investigation of cellular communications [1-2], mechanobiology [3-6] and development [5-7], and cell isolation [8]. PDMS has shown to carry numerous properties that offer advantages over many other available biomaterials such as silicon, bio glass and other polymers. For instance, an available master allows PDMS to be molded easily into sub-micron designs as defined by the master [1-8]. Furthermore, the elastomeric properties of the PDMS can be easily tuned by the base:curing agents ratio to cover a wide range of physiologically relevant elastic modulus [9] for mechanobiological studies as compared to other materials used for similar purpose, such as polyacrylamide gels [10-11], polyethylene glycol [12], and hyaluronan [13]. The surface roughness of the PDMS can also be easily adjusted with different curing temperatures [14]. These inherent properties of PDMS allow the study of physical effects on cell behavior especially in the field of stem cell differentiation. Adding to these advantages are their optical transparency, gas permeability, non-toxicity and cost effectiveness, which could make PDMS as a preferred material for cell-based platforms, used in biomedical devices and fundamental studies.

However, the surface compatibility of PDMS for cell culture is often discouraging [15-18]. One of their major drawbacks lies in the high surface hydrophobicity, which limits the affinity for mammalian cells to effectively adhere to the native PDMS surface [15-18]. Since surface 
wettability is one of the crucial factors in the influence of cell adhesion on the substrate [19-20], previous research has focused extensively on the modification of the PDMS surface to reduce its hydrophobicity [21-23]. While surface hydrophobicity of PDMS can be reduced by surface treatment with plasma [21], hydrophobic recovery was observed after plasma treatment [22-23]. Another approach is protein adsorption to PDMS substrate, which has been one of the widely-used techniques to facilitate cell adhesion due to their intrinsic biocompatibility with proteins and molecular recognition properties [1, 4-7]. However, the maintenance of cell adhesion on these surfaces was transitory whereby cells were either detached after cell confluence or aggregated to form loosely bound cell clumps [15].

Adhesion and aggregation of proteins on the material surface during protein adsorption rely on the interaction between the charged domains on the protein and the material surface [24]. These interaction forces (e.g. electrostatic, Van der Waals forces and hydrophobic interactions) are usually weak [25] and thus highly susceptible to protein leaching into the medium, thereby reducing the biocompatibility of the protein coated PDMS for cell culture. Efforts have been made to induce strong and stable covalent linkage of the protein to the material surfaces [26-28]. Among the covalent binding strategies, material surfaces chemically modified with amino-silane (e.g. (3aminopropyl) triethoxysilane (APTES)) and homo-bifunctional aldehyde (e.g. glutaraldehyde (GA)) chemistry have shown their efficiency in immobilizing proteins and antibodies [26-28]. APTES and GA activation function as molecular spacers to minimize the direct and weak interactions of proteins with the PDMS surface and overcome steric hindrance from the vicinity of the support, which is essential for stronger protein attachment [29]. Such chemistry in achieving stable covalent attachment of several proteins has been reported in applications such as immunoassay. However, the use of this chemical modified PDMS surfaces to stabilize cell adhesion and support cell proliferation has yet to be well investigated. It is thus of interest to extend its use to study the interaction of stable extracellular cellular matrix (ECM) protein activated surfaces with the adherent cells. In this regard, the efficiency of these APTES \pm GA 
modified PDMS surfaces in immobilizing common ECM proteins such as collagen type $1(\mathrm{C} 1)$ or fibronectin (FN), and the biocompatibility of these chemically modified surfaces for the adhesion and proliferation of mammalian cells remain unknown. Hereby, we foresee a need to investigate the applicability of PDMS with a well-defined surface chemistry for cell culture applications, and hypothesize that stable attachment of ECM proteins through covalent bonding can facilitate stronger cell adhesion and subsequently influence cell behavior.

Mesenchymal stem cell (hMSC) is a promising cell source for regenerative medicine due to their multipotency, and readily availability from mesenchymal origins. Specifically, in this study, for the first time to our best knowledge, we investigated the effect of the modified PDMS surfaces by APTES \pm GA cross-linking chemistry with the attachment of either FN or C1 on hMSC culture.

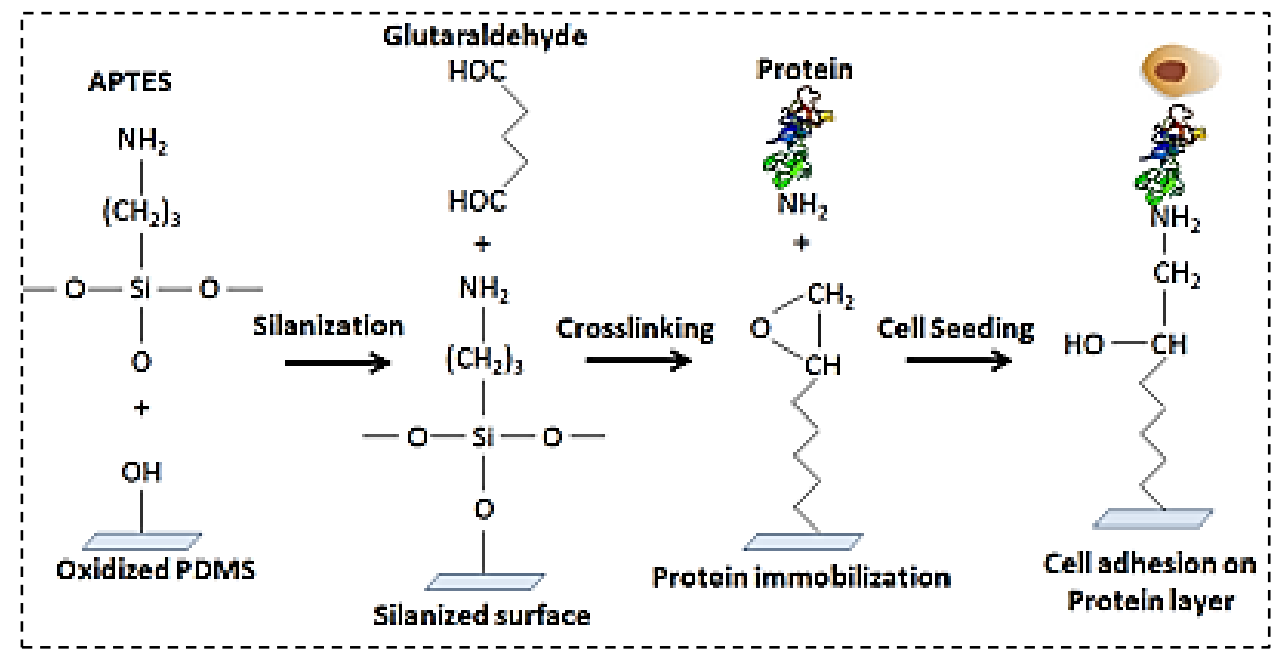

Figure 4-1: Schematic diagram of PDMS surface modification

APTES was first bound onto the plasma activated PDMS surface, followed by activation with GA. ECM proteins $(\mathrm{C} 1$ or $\mathrm{FN})$ were bound to the substrate surface modified either through APTES or APTES+GA routes (Figure 4-1). Surface characterization of the chemically modified PDMS surface was performed, and its bio-compatibility with the hMSCs was then assessed with appropriate analytical techniques. We demonstrated that this approach of chemical modification 
on the PDMS surfaces offered an improved biocompatible platform that enhanced cell adhesion and proliferation, which were critical for later stage of cell physiological development. 


\subsubsection{Results}

\subsubsection{X-ray Photoelectron Spectroscopy (XPS)}

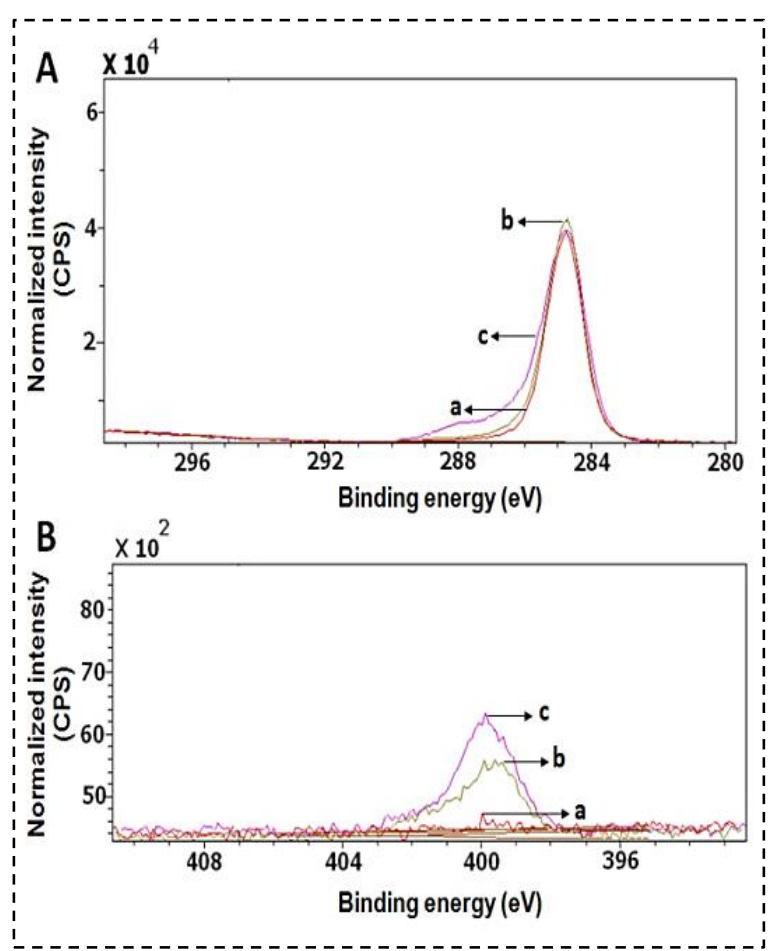

Figure 4-2: (A) C1s (carbon) and (B) N1s (nitrogen) region XPS scan on (a) Native PDMS, (b) PDMS+APTES and (c) PDMS+APTES+GA samples.

Surface elemental composition was obtained from XPS characterization. From Figure 4-2, it is seen that the relative loading of carbon (C1s) increased slightly in APTES and subsequently in APTES+GA modified samples as compared to the plain PDMS. Similarly, higher loading of nitrogen (N1s) was seen in both APTES and APTES+GA modified samples due to the presence of amine group (-NH2) of the organo-silane. 


\subsubsection{Surface wettability of modified/unmodified PDMS surfaces}

The surface wettability of the native PDMS, Tissue Culture Plate (TCP), chemically modified and unmodified PDMS with protein coating were evaluated by the measurement of water droplet contact angle on the substrate (Figure 4-3).

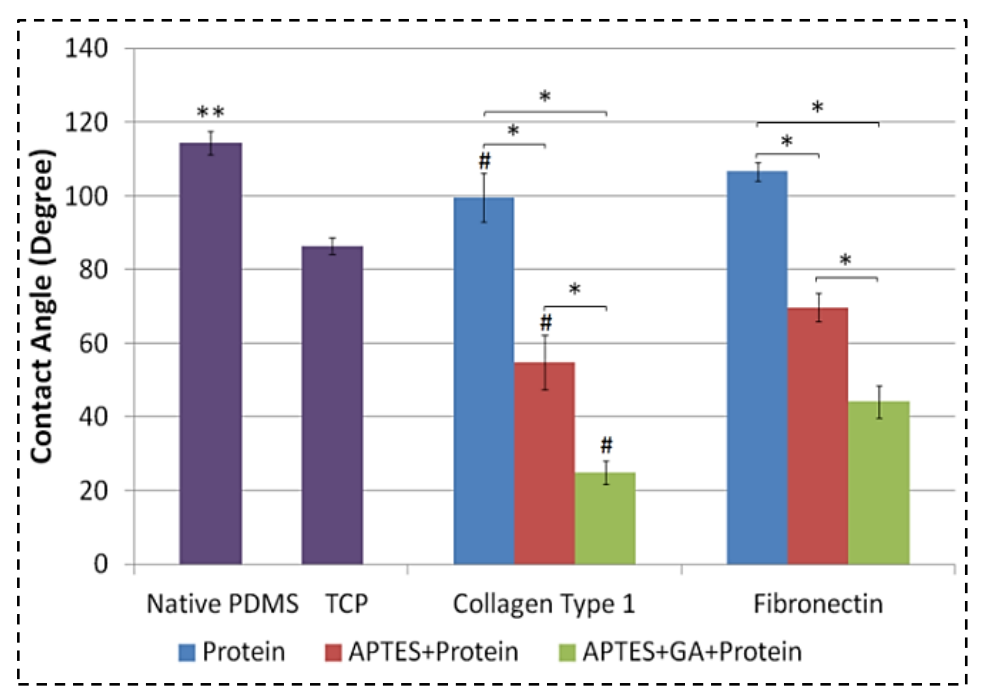

Figure 4-3: Average contact angle on TCP, and different PDMS surfaces. Data are shown as means \pm SD. *p value $<0.05$ between two groups. **indicates $p$ value $<0.05$ as compared to the rest of the groups. \#p value $<0.05$ as compared to fibronectin coated on the similar PDMS modified surfaces.

The wettability of the native PDMS was within the hydrophobic region $\left(114.41 \pm 3.14^{\circ}\right)$. While protein adsorption on the unmodified PDMS surface resulted in slight decrease of the contact angle, the surface wettability remained in the hydrophobic regions $\left(\sim 100^{\circ}\right)$. On the other hand, chemical modification of the PDMS surface by APTES and/or GA with protein adsorption could further reduce the contact angle to a hydrophilic region $\left(<70^{\circ}\right)$, for which the collagen type 1 adsorption showed significantly greater effect than the fibronectin adsorption.

\subsubsection{Protein immobilization}

Micro-BCA assay was used to evaluate the extent of protein immobilized on the modified/unmodified PDMS surfaces (Figure 4-4). 


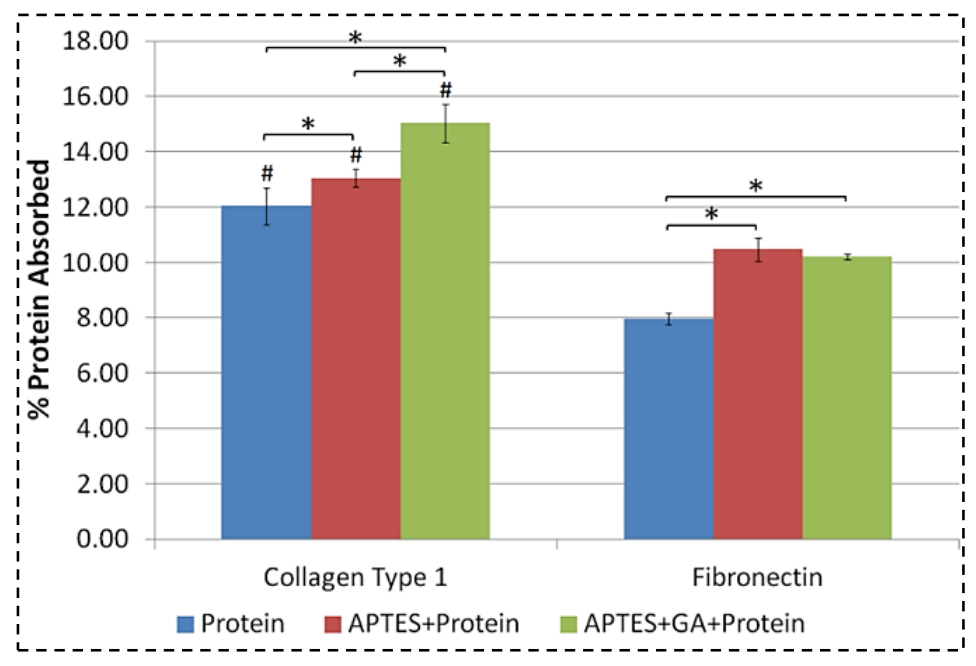

Figure 4-4: Amount of protein (collagen type 1 or fibronectin protein) retained on the modified/unmodified PDMS surfaces after one day of incubation. Data are shown as mean \pm SD. *p value $<0.05$ between two groups. \#p value $<0.05$ as compared to fibronectin coated on the similar PDMS modified surfaces.

The assay revealed that chemical modifications indeed enhanced the adsorption for both proteins. However, the attachment of collagen type 1 protein was significantly higher as compared to fibronectin attachment on similar PDMS surfaces.The adsorption of fibronectin, on the other hand, was enhanced in the APTES+Protein group but had a weaker affinity with the PDMS surface in APTES+GA+Protein group. The data also revealed the significant difference in the attachment of both proteins on similar PDMS surfaces.

\subsubsection{Characterization of cell adhesion on modified/unmodified PDMS surfaces}

To assess the influence of the chemically modified/unmodified PDMS surfaces on the adhesion of hMSCs, the amount of adherent cells were determined by CyQUANT® Cell Proliferation Assay Kit after 90 min of initial cells seeding. The adherent cell density was then expressed as a fold difference relative to that of the TCP group. Cell adhesion on PDMS modified with APTES, GA and protein was significantly higher than the rest of the groups (Figure 4-5). Further to that, 
fibronectin adsorption on any PDMS surfaces was shown to provide better cell adhesion as compared to collagen type 1 adsorption on similar PDMS surfaces (Figure 4-5).

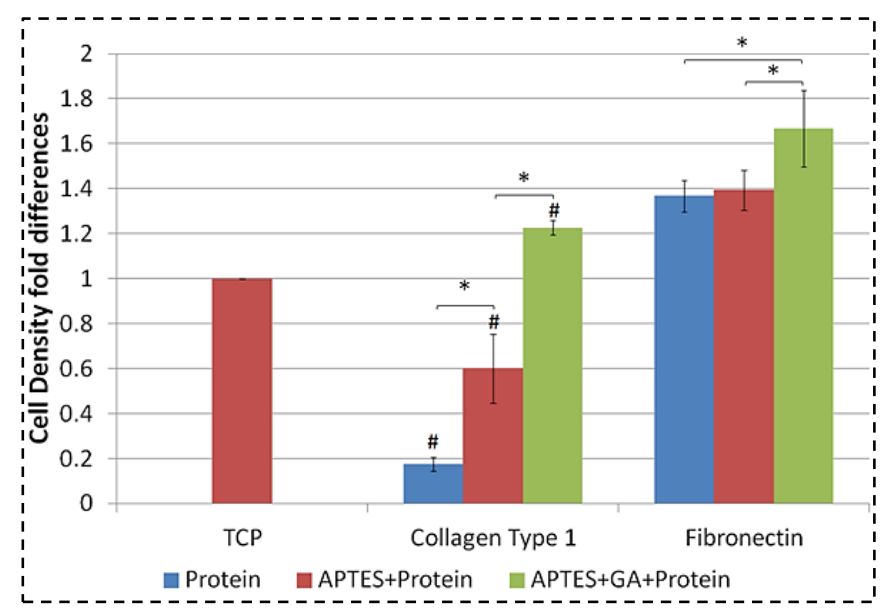

Figure 4-5: Adherent cell density after 90 min of initial hMSC seeding on modified/unmodified PDMS surfaces with either collagen type 1 or fibronectin protein coating. Cell density was presented as fold differences relative to the cell density on TCP. Data are shown as means \pm SD. *p value $<0.05$ between two groups. \#p value $<0.05$ as compared to fibronectin coated on the similar PDMS modified surfaces.

The hMSCs were further examined for their spread area on the different chemically altered PDMS surfaces 4 hours after initial cell seeding by TRITC-conjugated phalloidin staining and viewing with an inverted fluorescent microscope.

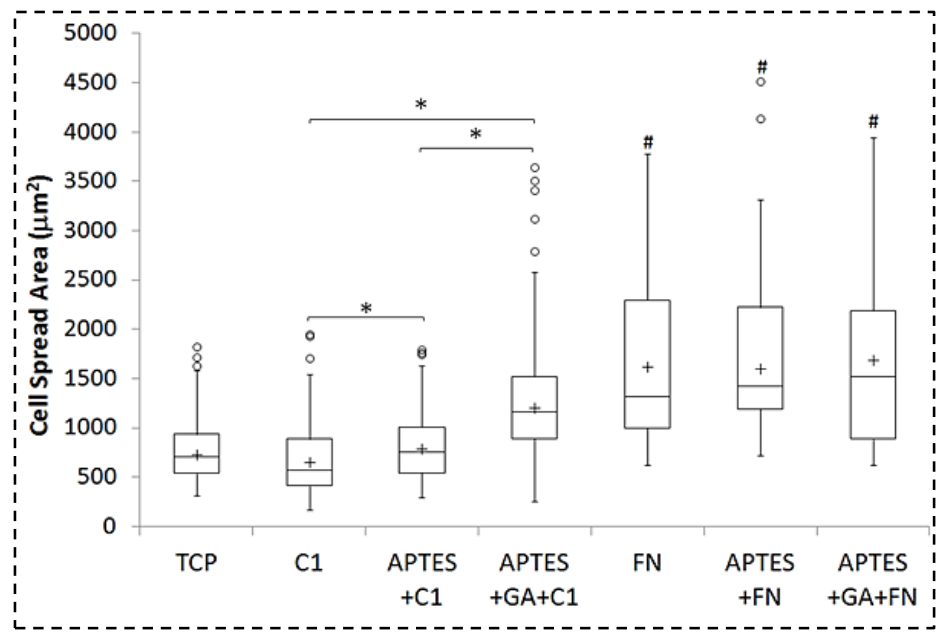

Figure 4-6: Mean and median cell spread area on modified or unmodified PDMS surface displayed in Box and Whiskers plots $(\mathrm{n}>30)$. Mean data are represented by + . *pvalue $<0.05$ between two groups. \#p value $<0.05$ as compared to collagen type 1 coated on the similar PDMS modified surfaces. 
The spread area of each single cell was measured by the Image-Pro Plus software. For the PDMS surfaces with collagen adsorption, cell adhesion on APTES+GA+protein resulted in a significantly higher spread area compared to the other two modified/unmodified PDMS surfaces (Figure 4-6 and Figure 4-7).

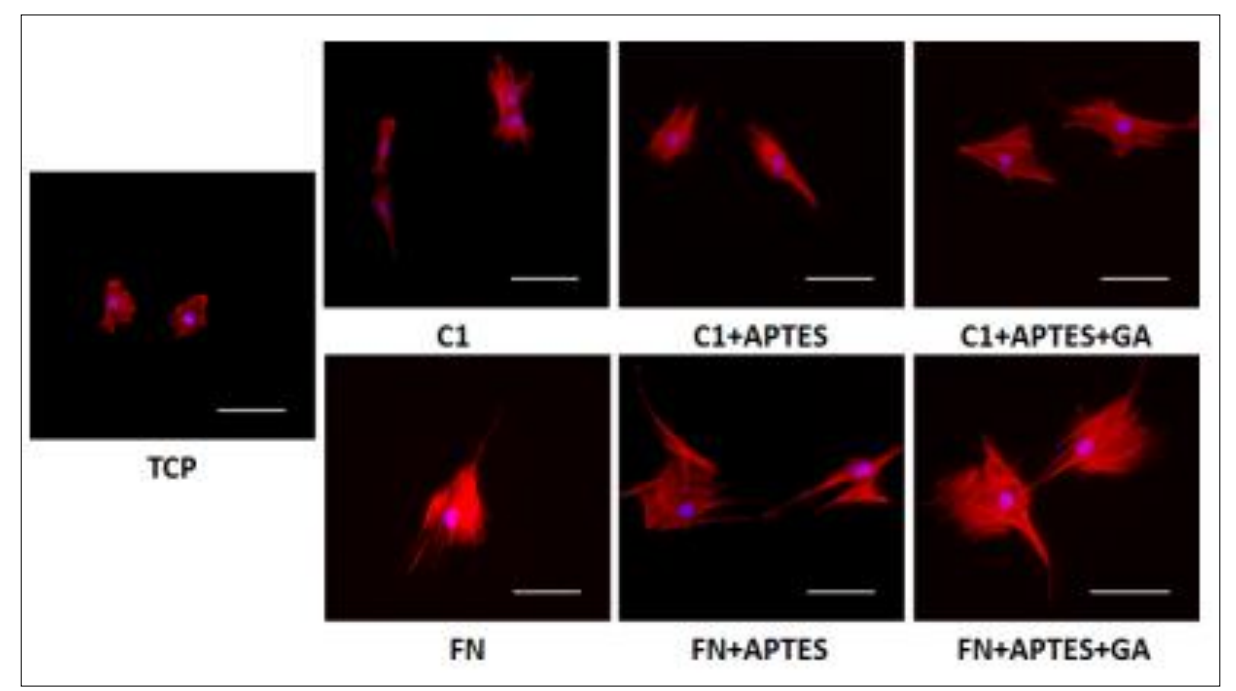

Figure 4-7: Cell-spread area of hMSCs on modified/unmodified PDMS surfaces. The actin cytoskeleton of hMSCs was stained red, and the cell nucleus was stained blue (Scale bar, $50 \mu \mathrm{m}$ ) with PrestoBlue Cell Viability Reagent for a duration of 14 days.

However, for PDMS surfaces with fibronectin adsorption, there was no significant difference in the spread area of the cells on all three modified/unmodified PDMS surfaces, while in general the cells spread much wider compared to the adhered hMSCs on TCP surfaces.

\subsubsection{Proliferation of cells on different chemically modified/unmodified PDMS surfaces}

The proliferation of hMSCs on different chemically modified/unmodified PDMS surfaces was evaluated with PrestoBlue ${ }^{\mathrm{TM}}$ Cell Viability Reagent for duration of 14 days. On the modified PDMS surfaces with collagen adsorption, it was observed that hMSCs remained metabolically 
active and continued to proliferate; meanwhile, hMSCs showed higher proliferation on the APTES+GA+Col1 surface (Figure 4-8a).

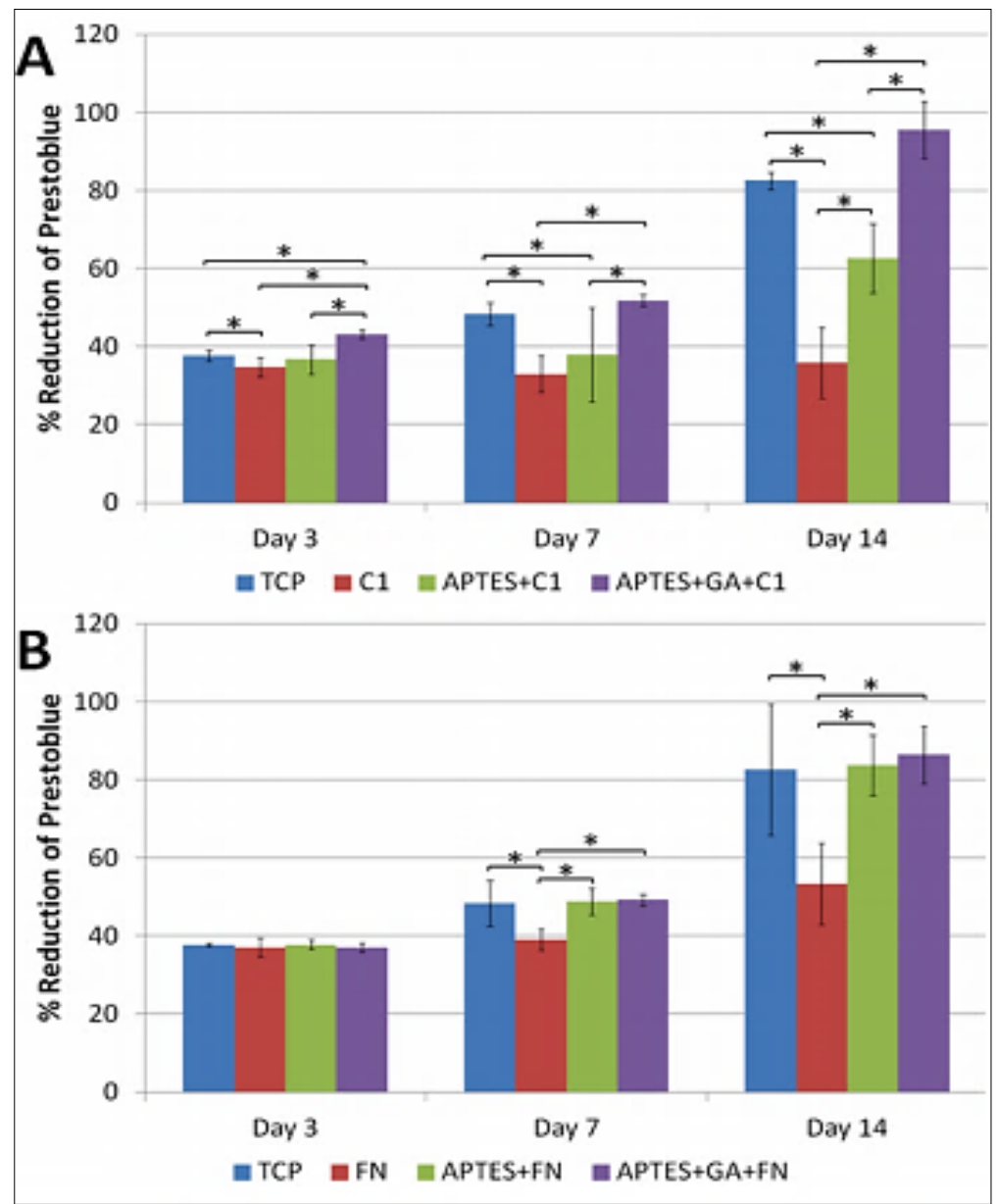

Figure 4-8: PrestoBlue assay for the proliferation of hMSCs cultured on PDMS-modified/unmodified surfaces with (A) collagen-1 or (B) fibronectin coating at 3, 7, and 14 days after seeding. *p value of $<0.05$.

On the other hand, hMSCs that were adhered to the unmodified PDMS surfaces with collagen adsorption remained metabolically inactive (Figure 4-8 A). For the modified/unmodified PDMS with fibronectin adsorption, there were no significant difference in Prestoblue dye reduction on day 3 but the subsequent data revealed significant difference in their proliferation activities on day 7 and 14, when the hMSCs exhibited enhanced proliferation activities on the modified PDMS surfaces as compared to the unmodified surfaces (Figure 4-8 B). 


\subsubsection{Discussion}

In recent years, the exploration of the cell-surface interaction has been gaining importance especially in biomaterial research for therapeutics, diagnostics and regenerative medicine. Investigating the effects of surface chemical modifications on cellular behavior is critical to assess optimal conditions for cell adhesion, expansion and proliferation. This requires a thorough understanding on surface functionalities and cell-surface interactions, which beneficially influence cell anchorage on the chemically modified substrates.

High hydrophobicity of PDMS surface and poor physical adsorption of ECM components, such as fibronectin, has commonly caused early detachment of cells, and uneven layering and clumping of loosely bound cell population, thereby limiting the use of PDMS in cell culture [15-18]. This could be attributed to the relative instability of the physically adsorbed surface proteins that are vulnerable to detachment. A significant loss of bioactivity through disruptions of the protein's conformation or weak attachment is bound to occur in subsequent washing or media changing steps [30] or even during routine perfusion process. In this study, PDMS was functionalized with APTES and GA cross-linking chemistry, which provided -NH2 and -CHO functional groups, respectively, to achieve a stable covalent bonding of collagen type 1 or fibronectin. $\mathrm{C} 1 \mathrm{~s}$ region scan data from XPS revealed the presence of higher percentage of carbon in the successive modification steps (Figure 4-2). This confirmed the modification of PDMS surfaces with APTES and GA due to the presence of triethyl $\left(-\mathrm{CH}_{2}\right)$ and aldehyde (-CHO) groups, respectively. Similarly, the N1s region scans revealed the presence of higher percentage of nitrogen in the silanized surface due to the presence of the amine group $\left(-\mathrm{NH}_{2}\right)$ of the APTES molecules. Subsequently, surface characterization and biocompatibility analysis of the chemically modified PDMS surfaces were performed to investigate the feasibility of the mentioned surface chemistry for application of cell culture studies.

Cell adhesion is vital for viability and growth, and its adhesion relationship with the substrate is usually influenced by the substrate surface properties, such as biomolecular/biochemical 
composition, wettability, charges and chemistry [31-32]. Therefore, it was of fundamental interest to investigate the level of cell attachment and spread area on the unmodified/modified PDMS surfaces. Surface wettability of the substrates has known to be one of the determining factors that influence cell adhesion. To relate the surface wettability of our functionalized surfaces to the influence of cell adhesion and behavior, the hydrophobicity of the unmodified/modified PDMS surfaces were assessed by the measurement of water contact angle. Previous studies have reported that PDMS surface functionalized with APTES and/or GA could result in a more hydrophilic surface [33-34]. These reported phenomena were observed to be consistent with the water contact angle measurements performed on the APTES \pm GA modified surfaces (data not shown), in which the reduced hydrophobicity could be attributed to their hydrophilic carbonyl and amine functional groups. Physical adsorption of protein alone (without any surface chemical treatment) could reduce the hydrophobicity of the native PDMS, but the surfaces were still hydrophobic $\left(>90^{\circ}\right)$ (Figure 4-3). On the other hand, protein attachment on APTES+GA surfaces had proved to be the most effective chemical modification to render hydrophilic surface among the three experimental groups, with collagen type 1 bound surfaces more hydrophilic than the fibronectin bound surfaces (Figure 4-3). Despite of the higher wettability on collagen bounded surfaces than on fibronectin bounded surfaces, cell adhesion was reported to be weaker on collagen bounded surfaces (Figure 4-5). These suggested that the cell adhesion to a substrate surface was determined not only by its wettability, but also by the types of proteins or functional groups present on the substrate surfaces. Cell interaction with ECM proteins are vital to many biological processes [35-36], and in numerous studies, matrix proteins such as fibronectin and collagen, have been coated on substrate for in vitro cell based experiments [1,4-7]. Hence, it is essential to generate stable attachment of matrix proteins by surface chemical functionalization. Past studies have shown that chemical modification leads to a higher surface stability than physical adsorption alone [26-28], which could probably result in an enhanced biocompatibility for cell culture. To assess the stability of proteins bound to each unmodified/modified surface, Tween 20 was used to remove loosely bound 
proteins. The formation of stable protein-substrate covalent linkage had resulted in the higher amount of protein being strongly attached on the chemically modified PDMS (Figure 34). Meanwhile, it was observed that collagen type 1 had a greater affinity than fibronectin on the unmodified/modified PDMS surfaces. This could be attributed to the differences in their amino acid composition and protein conformation that influence the intermolecular forces between the proteins and the substrates, which would facilitate the attachment of proteins [37]. Functionalizing APTES and GA cross-linkers on the PDMS surface offers the possibility for preferential orientation and covalent attachment of proteins through their respective affinity moieties that bind to the reactive functional groups [38].

We observed that surfaces coated with collagen type 1 had an increased wettability (Figure 4-3) with a higher amount of absorbed proteins (Figure 4-4) than those coated with fibronectin, which could be favorable conditions for cell adhesion. However, the comparison of actual hMSC adhesion on fibronectin and collagen type 1 showed an opposite trend. We compared the level of cell adhesion and cell spreading on unmodified/modified surfaces with different protein adsorption, and the findings revealed relatively lower level of cell adhesion and spreading on all the collagen adsorbed surfaces as compared to fibronectin adsorbed surfaces. These results suggested that besides surface wettability and the underlying immobilization chemistry, the biomolecules (proteins) present on the surfaces also influenced the level of cell adhesion and spreading. Many studies have reported that cell adhesion on collagen coated surface was inferior to those on fibronectin coated surfaces [32-41].

The presence of cell adhesion motifs in ECM proteins $[32,42]$ and the multiple integrin receptors on cells with different ligand specificities [43] can mediate cell adhesion to different matrix proteins. Additionally, protein conformation on the substrate surfaces could also contribute to differential affinity for cell adhesion on different protein coated surfaces [44]. The fibronectin attachment on APTES coated substrates are higher compared too plain PDMS due to enhanced 
ionic level interaction of charged amine group of the APTES layers with the protein. Moreover, APTES treated surfaces are relatively less hydrophilic than the plain PDMS surface.

The cell attachment data shown in Figure 4-5 shows a comparison of the strength of initial cell adhesion on the substrates within 90 min of cell seeding. In this short duration, the cell membrane interaction on the surfaces (modified/unmodified) are mostly via temporary electrostatic interactions and partly due to ionic interactions due to which on the plain PDMS+FN surface, higher initial adhesion of cell membrane proteins could be expected. The initial cell adhesion on APTES+FN substrates is comparable and only slightly higher than the plain PDMS+FN surfaces perhaps due to relatively hydrophilic nature of substrate which can positively influence focal adhesion and cell anchorage [46].

However, the trends in terms of the attached cell number varied significantly with time as seen in the subsequent proliferation data (Figure 4-8.) The cell proliferation remained almost constant for plain PDMS+FN substrates throughout the experimental time span which indicates poor cell adhesion and eventual cell loss from substrates. This shows that the FN coating on plain substrates can only support initial adhesion due to temporary interactions. On the contrary, the APTES layer has relatively higher functional level interactions and cell adherence which promotes active cell proliferation.

Arima et. al.[31] reported that functional groups expressed on the substrate surface had an impact on the adhesion of cells, where amino groups encouraged better adhesion of endothelial cells than hydroxyl and carboxylic groups. In our study, chemically modified surfaces with either protein coating improved the cell adhesion (Figure 4-5). The effect on cell spreading for FN coated PDMS surfaces however is not as notable as those on C1 coated PDMS surfaces (Figure 4-6 and Figure 4-7). All the above-mentioned phenomena suggested that the enhanced hMSC adhesion/spreading on APTES+GA+Protein surfaces could be attributed to the synergistic effects of multiple contributing factors, such as the increased wettability of the surfaces, influence of reactive 
functional groups in APTES/GA on protein attachment/conformation, and the choice of matrix protein, given their preferential affinity.

Protein adsorption on substrate surface, cell-substrate surface adhesion, and cell spreading were a few critical steps that preceded cell proliferation in the later stages. Besides, a study by Chen et al. showed that promoted cell spread area could potentially lead to an enhanced proliferation of the cells [45]. The cell proliferation on each unmodified/modified PDMS surfaces was thus evaluated with Prestoblue assay, and correlated with results of the cell spread area as discussed earlier. On collagen coated surfaces, it was shown that the spreading area and proliferation of hMSCs were both significantly higher on PDMS functionalized with APTES+GA (Figure 4-6 and Figure 4-8A); while there were no significant hMSC growth observed on unmodified PDMS surface over the 14 days. On the other hand, hMSC spreading area measured on fibronectin coated surfaces showed no much difference among the three experimental surfaces (Figure 4-6 and Figure 4-7). However, enhanced hMSC proliferation was observed on fibronectin coated surfaces functionalized with APTES \pm GA (Figure 4-8B). As hMSCs were cultured on different functionalized surfaces, these observations suggested that hMSC proliferation could not be attributed only to the extent of cell spreading, which implied that other factors, such as the surface chemistry, wettability and the protein types, could also potentially influence the hMSC proliferation.

The drawback of native PDMS was unfavorable to cell culture. While physical adsorption of ECM protein might help in initial cell adhesion, early detachment of cells and cell clumping were observed during cell confluence or even before reaching confluency [15-18]. Further, this proved disadvantageous for long term cell based applications, such as tissue engineering works. Our study demonstrated that PDMS surface functionalized with APTES+GA+protein adsorption provided a biocompatible surface for cell based experiments. Our data showed that chemical modification not only provided a hydrophilic surface but also showed a stable covalent linkage of proteins, which was a prior step to establish strong anchorage of cells. Further, strongly adhered hMSCs could 
have a great potential in long term cell based studies. Future experiments will be directed towards demonstrating the versatility of this surface treatment for long term culture. Since these surfaces offer a convenient platform for long term studies on hMSCs, analytical validation could be performed to evaluate the functionality characterization of hMSCs on these functionalized surfaces.

\subsubsection{Conclusion}

PDMS surfaces functionalized with APTES \pm GA and the attachment of either $\mathrm{C} 1$ or FN was used in this study to demonstrate the ability of modified PDMS to serve as a biocompatible substrate for long-term cell culture. Chemical functionalization was shown to reduce the hydrophobicity of the native PDMS surfaces. APTES and GA cross-linking chemistry showed stable covalent attachment of the matrix proteins based on the available active surface functional groups. The notable enhancement of hMSC adhesion, spread area, and proliferation were best observed on the PDMS surfaces functionalized with APTES + GA + proteins. For C1- coated surfaces, the chemical modification improved cell adhesion, spreading, and proliferation significantly. For FN coated surfaces, although the promotion of cell spreading is not as notable as $\mathrm{C} 1$ groups, the chemical modification did greatly enhance the cell proliferation. However, in general, the hMSCs demonstrated a higher affinity, in terms of cell adhesion and spreading, on FN-coated PDMS than on $\mathrm{C} 1$ coated PDMS. These results indicated that surface chemical modification with APTES + GA + proteins had a profound effect on cell adhesion, spread area, and proliferation in which the synergistic effects of multiple contributing factors, such as surface wettability, protein matrix, and APTES/GA functional groups, played a combined role. We reported the utility of biocompatible PDMS surfaces functionalized with APTES + GA + proteins for enhanced cell culture stability, which can be potentially used for the study of cellular physiology, especially in tissue engineering and mechanobiology 


\section{References}

[1] Chung S, Sudo R, Mack PJ, Wan CR, Vickeman V, Kamm RD. Cell migration into scaffolds under co-culture conditions in a microfluidic platform. Lab Chip 2009; 9:269275.

[2] Zhang Q, Liu T, Qin J. A microfluidic-based device for study of transendothelial invasion of tumor aggregates in realtime. Lab Chip 2012; 12:2837-2842.

[3] Park JY, Yoo SJ, Lee EJ, Lee DH, Kim JY, Lee SH. Increased poly(dimethylsiloxane) stiffness improves viability and morphology of mouse fibroblast cells. BioChip J 2010; $4: 230-236$.

[4] Wu MH, Wang HY, Liu HL, Wang SS, Liu YT, Chen YM, Tsai SW, Lin CL. Development of high throughput perfusion-based microbioreactor platform capable of providing tunable dynamic tensile loading to cells and its application for the study of bovine articular chondrocytes. Biomed Microdevices 2011; 13:789-798.

[5] Fu J, Wang YK, Yang MT, Desai RA, Yu X, Liu Z, Chen CS. Mechanical regulation of cell function with geometrically modulated elastomeric substrates. Nat Methods 2010; 7:733-736.

[6] Yim EKF, Pang YT, Leong KW. Synthetic nanostructures inducing differentiation of human mesenchymal stem cells into neuronal lineage. Exp Cell Res 2007; 313:18201829.

[7] Kilian KA, Bugarija B, Lahn BT, Mrksich M. Geometric cues for directing the differentiation of mesenchymal stem cells. Proc Natl Acad Sci 2010; 107:4872-4877.

[8] Carlo DD, Aghdam N, Lee LP. Single-cell enzyme concentrations, kinetics, and inhibition analysis using high-density hydrodynamic cell isolation arrays. Anal. Chem. 2006; 78:4925-4930.

[9] Palchesko RN, Zhang L, Sun Y, Feinberg AW. Development of polydimethylsiloxane substrates with tunable elastic modulus to study cell mechanobiology in muscle and nerve. PLoS ONE 2012;7: e51499.

[10] Sunyer R, Jin AJ, Nossal R, Sackett DL. Fabrication of hydrogels with steep stiffness gradients for studying cell mechanical response. PLoS ONE 2012; 7:e46107.

[11] Fischer RS, Myers KA, Gardel ML, Waterman CM. Stiffness-controlled threedimensional extracellular matrices for high-resolution imaging of cell behavior. Nat Protocols 2012; 7:2056-2066.

[12] Peyton SR, Raub CB, Keschrumrus VP, Putnam AJ. The use of poly (ethylene glycol) hydrogels to investigate the impact of ECM chemistry and mechanics on smooth muscle cells. Biomaterials 2006; 27:4881-4893.

[13] Rehfeldt F, Brown AE, Raab M, Cai S, Zajac AL, Zemel A, Discher DE. Hyaluronic acid matrices show matrix stiffness in $2 \mathrm{D}$ and $3 \mathrm{D}$ dictates cytoskeletal order and myosin-II phosphorylation within stem cells. Integr Biol (Camb) 2012; 4:422-430.

[14] Ortiz GC. The Study Of Polydimethylsiloxane (Pdms) Thin-Film Surface Roughness Properties And Its Resistance In Elevated Temperature And Uv Light Resistant Applications. URL: ttp://urpasheville.org/proceedings/ncur2011/papers/NP51596.pdf (Last accessed April 2013)

[15] Lee JN, Jiang X, Ryan D, Whitesides GM. Compatibility of mammalian cells on surfaces of poly(dimethylsiloxane). Langmuir 2004; 20:11684-11691.

[16] Fuard D, Chevolleau TT, Decossas S, Tracqui P, Schiavone P. Optimization of poly-dimethyl-siloxane (PDMS) substrates for studying cellular adhesion and motility. Microelectronic Engineering 2008; 85:1289-1293.

[17] McDonald JC, Duffy DC, Anderson JR, Chiu DT, Wu H, Schueller OJA, Whitesides GM. Fabrication of microfluidic systems in poly(dimethylsiloxane). Electrophoresis 2000;21:27-40. 
[18] Duffy DC, McDonald JC, Schueller JA, Whitesides GM. Rapid Prototyping of Microfluidic Systems in Poly (dimethylsiloxane). Anal. Chem 1998; 70:4974-4984.

[19] Tamada Y, Ikada Y. Effect of preadsorbed proteins on cell adhesion to polymer surfaces. J Colloid Interface Sci 1993; 155:334-339.

[20] Lee JH, Khang G, Lee JW, Lee HB. Interaction of Different Types of Cells on Polymer Surfaces with Wettability Gradient. J Colloid Interface Sci 1998; 205:323-330.

[21] Tan HML, Fukuda H, Akagi T, Ichiki T. Surface modification of poly(dimethylsiloxane) for controlling biological cells' adhesion using a scanning radical microjet. Thin Solid Films 2007;515:5172-5178.

[22] Bodas D, Khan-Malek C. Hydrophilization and hydrophobic recovery of PDMS by oxygen plasma and chemical treatment-An SEM investigation. Sens Actuator B Chem 2007; 123:368-373.

[23] Hillborg H, Gedde UW. Hydrophobicity recovery of polydimethylsiloxane after exposure to corona discharges. Polymer 1998; 39:1991-1998.

[24] Yao X, Wu HX, Wang J, Qu S, Chen G. Carbon nanotube/poly(methyl methacrylate) (CNT/PMMA) composite electrode fabricated by in situ polymerization for microchip capillary electrophoresis. Chemistry-a European Journal 2007; 13:846-853.

[25] Lodish H, Berk A, Zipursky SL, Paul M, David B, James D. Molecular Cell Biology. 4th edition. New York: WH Freeman and Company; 2000.

[26] Yuan H, Mullett WM, Pawliszyn J. Biological sample analysis with immunoaffinity solidphase microextraction. Analyst 2001; 126:1456-1461.

[27] Halliwell CM, Cass AEG. A factorial analysis of silanization conditions for the immobilization of oligonucleotides on glass surfaces. Anal Chem 2001; 73:2476-2483.

[28] Wang ZH, Jin G. J. Covalent immobilization of proteins for the biosensor based on imaging ellipsometry. Immunol Methods 2004; 285:237-243.

[29] Deshpande K, Ahamed T, van der Wielen LA, Horst JH, Jansens PJ, Ottens M. Protein self-interaction chromatography on a microchip. Lab Chip 2009; 9:600-605.

[30] Macdonald C, Morrow R, Weiss AS, Bilek MM. Covalent attachment of functional protein to polymer surfaces: a novel one-step dry process. J R Soc Interface 2008; 5:663669.

[31] Arima $\mathrm{Y}$, Iwata $\mathrm{H}$. Effect of wettability and surface functional groups on protein adsorption and cell adhesion using well-defined mixed self-assembled monolayers. Biomaterials 2007; 28:3074-3082.

[32] Cooke MJ, Phillips SR, Shah DSH, Athey D, Lakey JH, Przyborski SA. Enhanced cell attachment using a novel cell culture surface presenting functional domains from extracellular matrix proteins. Cytotechnology 2008; 56:71-79.

[33] Lee M, Kim KH, Park JG, Lee JH, Lim HW, Park MY, Chang SI, Lee EK, Lim DW, Choo J. Fabrication of a hydrophobic/hydrophilic hybrid-patterned microarray chip and its application to a cancer marker immunoassay. BioChip Journal 2012; 6:10-16.

[34] Sandison ME, Cumming SA, Kolchbc W, Pitta AR. On-chip immunoprecipitation for protein purification. Lab Chip 2010; 10:2805-2813.

[35] Kleinman HK, Philp D, Hoffman MP. Role of the extracellular matrix in morphogenesis. Curr Opin Biotechnol 2003; 14:526-532.

[36] Adams JC, Watt FM. Regulation of development and differentiation by the extracellular matrix. Development 1993; 117:1183-1198.

[37] Heilshorn SC, Liu JC, Tirrell DA. Cell-binding domain context affects cell behavior on engineered proteins. Biomacromolecules 2005; 6:318-323.

[38] Wu Y, Buranda T, Metzenberg RL, Sklar LA, Lopez GP. Diazo coupling method for covalent attachment of proteins to solid substrates. Bioconjug Chem 2006; 17:359-365.

[39] Sengel P, Kieny M. Influence of collagen and fibronectin substrates on the behaviour of cultured embryonic dermal cells. Br J Dermatol 1984; 111:88-97. 
[40] Chen G, Kawazoe N, Tateishi T. Effects of ECM Proteins and Cationic Polymers on the Adhesion and Proliferation of RatIslet Cells. The Open Biotechnology Journal 2008; 2:133-137.

[41] Junker JL, Heine UI. Effect of adhesion factors fibronectin, laminin, and type IV collagen on spreading and growth of transformed and control rat liver epithelial cells. Cancer Res 1987; 47:3802-3807.

[42] Feng Y, Mrksich M. The synergy peptide PHSRN and the adhesion peptide RGD mediate cell adhesion through a common mechanism. Biochemistry 2004; 43:15811-15821.

[43] Clyman RI, McDonald KA, Kramer RH. Integrin receptors on aortic smooth muscle cells mediate adhesion to fibronectin, laminin, and collagen. Circ Res 1990; 67:175-186.

[44] Grinnell F, Feld MK. Fibronectin adsorption on hydrophilic and hydrophobic surfaces detected by antibody binding and analyzed during cell adhesion in serum-containing medium. J Biol Chem 1982; 257:4888-4893.

[45] Chen CS, Mrksich M, Huang S, Whitesides GM, Ingber DE. Geometric control of cell life and death. Science 1997; 276:1425-1428.

[46] Grinnell F, et al., Fibronectin adsorption on hydrophilic and hydrophobic surfaces detected by antibody binding and analyzed during cell adhesion in serum-containing medium. J Biol Chem 1982; 257:4888-4893. 


\subsection{Effect of surface silanization on long term stability and fate of hMSCs}

\subsubsection{Background}

Poly (dimethyl siloxane) (PDMS) has been increasingly exploited to develop biological platform or microfluidic devices for cell and tissue engineering applications due to the precision in controlling the micro-environment with minimal bio-reagent consumption and experimental costs [1]. While several other biomaterials can be considered for similar fabrication purpose, PDMS is still widely utilized for rapid prototyping in the exploratory stages of fundamental research due to its versatility, transparency, gas permeability, low cost and ease of fabrication [2-3]. However, the highly hydrophobic nature of PDMS often renders a non-biocompatible surface for long-term cell analysis [4-7]. Although many have improved the bio-compatibility of PDMS by oxygen plasma treatment to improve the surface wettability or protein coating to enhance cell adhesion, these effects are often transient. For example, hydrophobic recovery is often observed on plasma-treated PDMS surface depending on time and temperature [8-9]; and cell aggregation or clumping occurs on protein-coated PDMS when the cell population reaches confluence. These problems have limited the advancement of microfluidic technologies towards the development of microdevices for long-term cell studies. Therefore, there is a need for surface modification of the PDMS substrate to improve its bio-affinity for stabilized cell culture.

Surface properties play a pivotal role in mediating stem cell behavior, including adhesion, proliferation and differentiation [10-13]. It was found that silanization of PDMS could lead to the formation of a cross-linking self-assembled monolayer (SAM), which had proved to be an efficient strategy to stabilize the protein immobilization on PDMS in many studies [14-16]. Inspired by these early studies, we had recently demonstrated that chemical modification with (3aminopropyl) triethoxysilane (APTES) and glutaraldehyde (GA) could change the surface chemistry, wettability and protein binding affinity on the PDMS substrate (discussed in Chapter 
4.1) [17]. These changes had induced constructive and synergistic effects on cell adhesion, morphology, and proliferation of the hMSCs. While the chemical modification could enhance cell adhesion and proliferation in a short time frame, some important questions of this application related to tissue engineering remain unsolved. For example, it is still unclear if the chemical modification could stabilize the cell adhesion upon confluence, and how it could affect the potency of the adhered hMSCs during a long-term culture.

One of the major applications of hMSCs in regenerative medicine is their differentiation towards osteogenic lineage for further use in bone regeneration. Besides that, hMSCs have been recently exploited to develop continuous cell sheets as an alternative strategy to benefit stem cell-based tissue engineering, such as blood vessel [18], cartilage [19], bone [20], and myocardium [21]. To explore the effect of different cues (e.g. chemical/mechanical/growth factors) on the stem cell potency, long-term adhesion and stability for several weeks on the substrate surface is necessary for in vitro observation and analysis. 


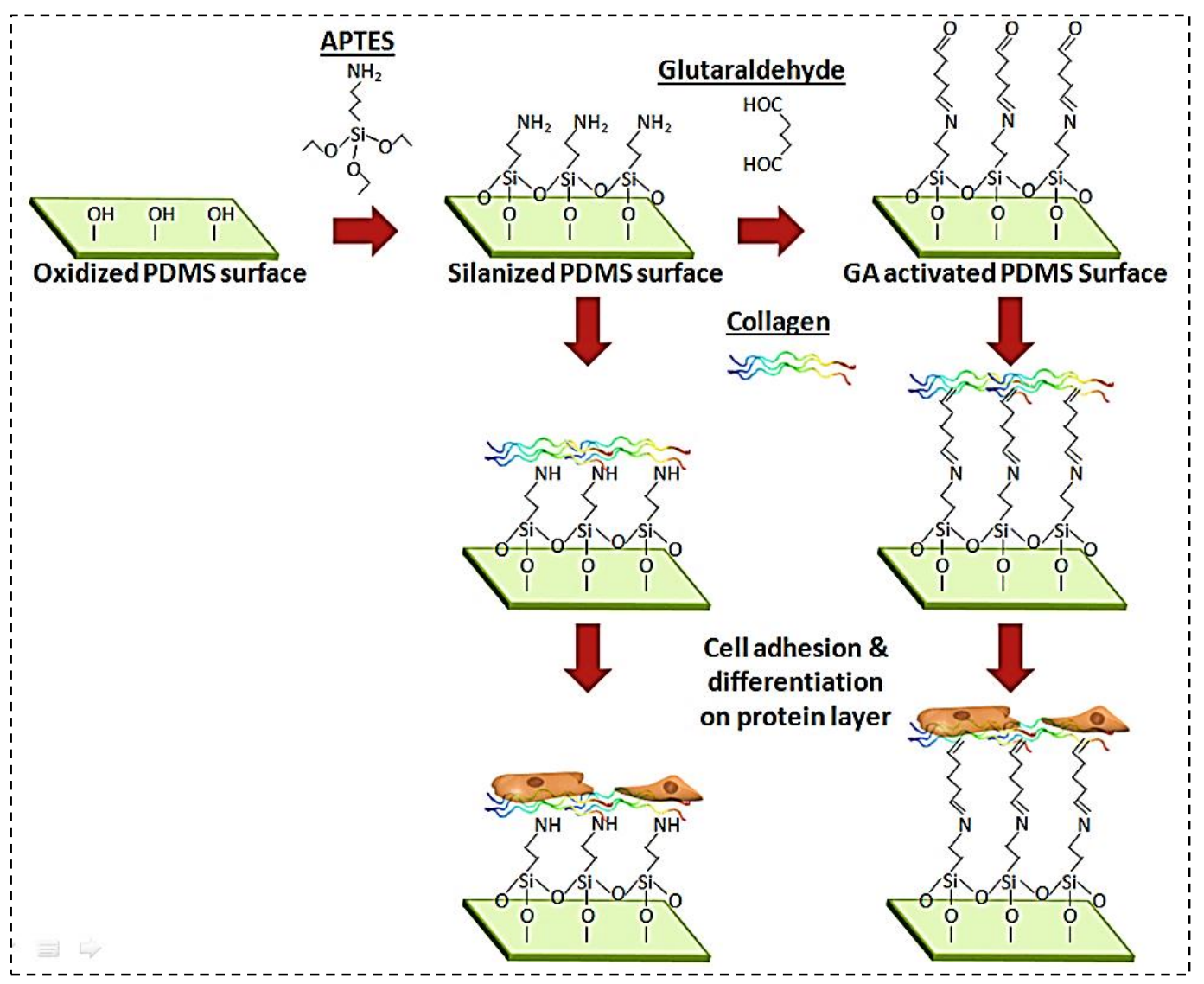

Figure 4-9: Schematic illustration of PDMS surface modification and subsequent cell culture.

Therefore, in this study, we performed PDMS surface silanization by APTES with/without GA, followed by protein immobilization using collagen type 1 (Col1), and evaluated the stability of confluent cell layer and the potency of adhered hMSCs for up to three weeks (Figure 4-9). In Chapter 4-1, the overall biocompatibility and hMSC anchorage on APTES+GA+ (FN or Col1) functionalized supports were tested. In this chapter, we specifically demonstrate the applicability of APTES+GA+Col1 substrates for long term cell sheet adhesion and subsequent osteogenic differentiation. Since Col1 is the most abundant protein in osteoblast micro-environments and osteogenic cell sheets synthesize Col1 during cell sheet growth, we used the covalent linking of Col1 as a model to test hMSC potency on the chemically modified PDMS. In subsequent chapters (Chapter 4.2 and 6), the applicability of Col1 immobilized substrates are specifically used in 
various chemistries for long term study of hMSCs viability, cell sheet stability and osteogenic differentiation in flat 2D, micro-chip and micro-fabricated supports (with multiscale features). 


\subsubsection{Results and Discussion}

In this study, PDMS surface was silanized with APTES+GA or APTES only prior to Col1 immobilization (Figure 4-9). The PDMS surface properties were then analyzed and their effect on the stability and potency of hMSCs was evaluated.

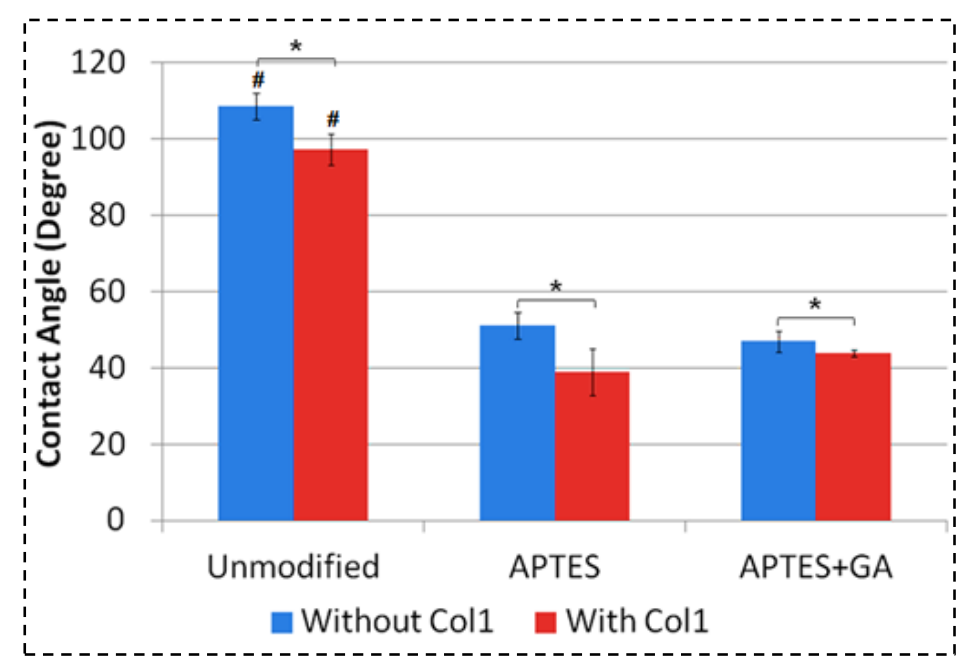

Figure 4-10: Average contact angles measured on unmodified and chemically modified PDMS surfaces, with and without Col1 coating. The data are shown as the mean \pm SD. *p value $<0.05$ between two groups under comparison. \#p value $<\mathbf{0 . 0 5}$, as compared to the modified PDMS surfaces with or without Col1 coating.

The surface wettability of the native PDMS as characterized by water contact angle exhibited a hydrophobic surface property $\left(108.63^{\circ} \pm 3.46^{\circ}\right)$. Although Col1 adsorption resulted in a slight decrease of contact angle, the surface of PDMS+Col1 remained hydrophobic $\left(97.33^{\circ} \pm 4.00^{\circ}\right)$. Surface silanization is known to significantly reduce the hydrophobicity of the PDMS surface,[17] as confirmed in this study, showing that PDMS silanization by APTES with/without GA could reduce the contact angle to hydrophilic region $\left(<60^{\circ}\right)$. Col1 protein adsorption on these chemically modified surfaces led to further reduction in contact angle $\left(<50^{\circ}\right)$ (Figure 4-10). 


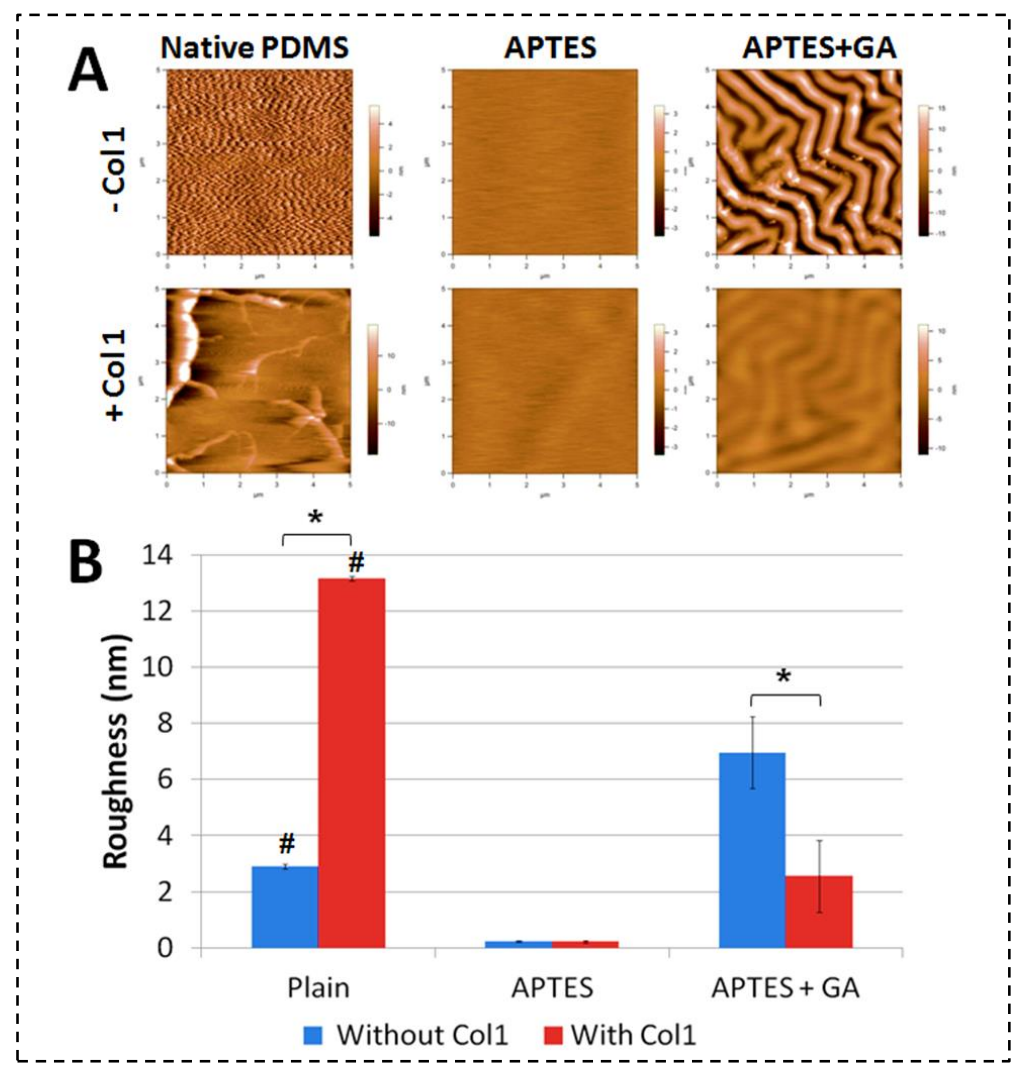

Figure 4-11: (A) Substratum topography and (B) RMS roughness of unmodified and chemically modified PDMS surfaces, with and without Col1 coating. *indicates a $p$ value $<0.05$ between two groups under comparison. \#p-value $<0.05$, as compared to the chemically modified PDMS surfaces, with/without Col1 coating.

The unmodified PDMS exhibited root-mean-square (RMS) roughness of $2.895 \pm 0.085 \mathrm{~nm}$, which increased notably to $13.17 \pm 0.68 \mathrm{~nm}$ when coated with Col1 protein (Figure 4-11a). The nonuniform substrate topography suggested protein aggregation on the unmodified PDMS surface. In contrast, the APTES-modified PDMS displayed the lowest roughness $(0.229 \pm 0.039 \mathrm{~nm})$, and there was no significant difference before and after Coll coating $(0.2184 \pm 0.01 \mathrm{~nm})$. Meanwhile, additional cross-linking with GA after silanization resulted in higher roughness $(6.952 \pm 2.555 \mathrm{~nm})$ with formation of evenly distributed nano-ridges. Coating of Col1 on the PDMS modified by APTES+GA further brought the roughness down to $2.555 \pm 0.35 \mathrm{~nm}$ without evident protein aggregation. 


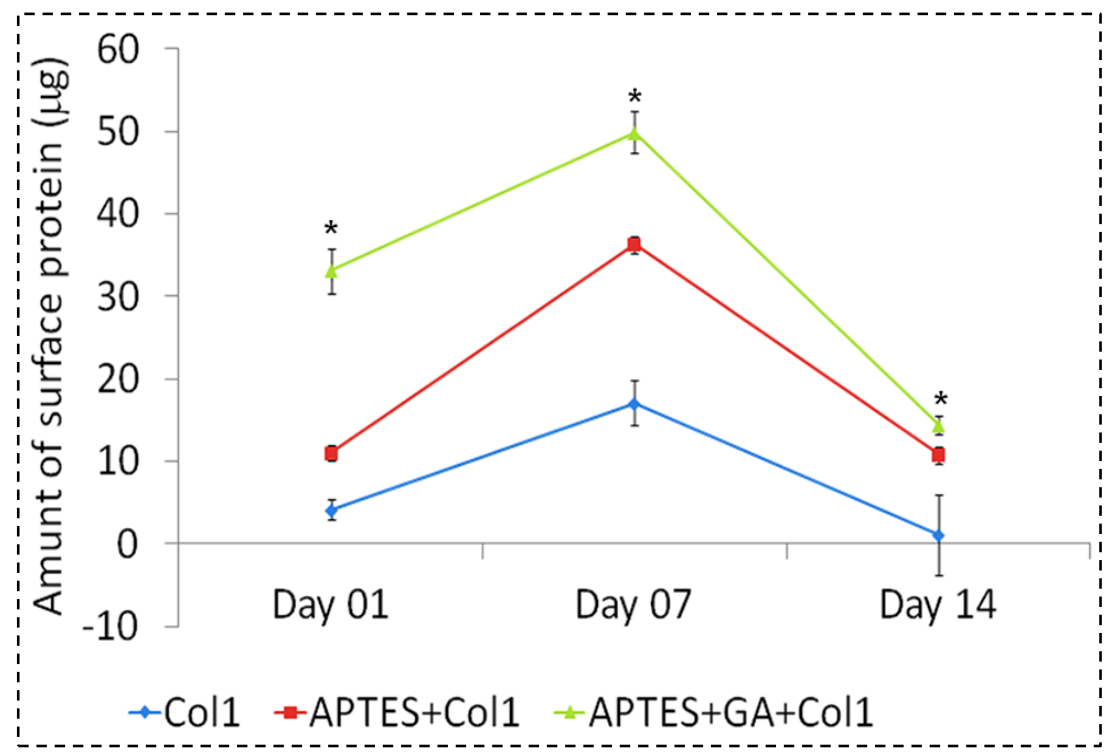

Figure 4-12: Amount of surface bound protein (Col1) measured on the unmodified (Col1) and chemically (APTES/ APTES+GA) modified PDMS surfaces on day 1, day 7 and day 14, respectively in cell incubating conditions. The data are shown as the mean \pm SD. *p value of $<0.05$ between any two groups.

Silanization of PDMS surface with APTES with/without GA enables the formation of selfassembled monolayers of molecules with reactive functional groups $\left(-\mathrm{NH}_{2}\right.$ or $\left.-\mathrm{COOH}\right)$ and specific covalent binding with proteins $[14,16]$. Effective matrix protein immobilization on substrate is necessary to promote stable cell adhesion at the focal adhesion domains, thus aiding in long-term cell culture. Figure 4-12 shows the protein retention of Coll on unmodified and APTES/APTES+GA modified substrates quantified by the micro-BCA assay. The stability of the attached Col1 on the modified PDMS surfaces with APTES only or APTES+GA was investigated for up to 2 weeks. Higher amount of surface bound Col1 was observed on the chemically modified PDMS as compared to the native PDMS (Figure 4-12), which was attributed to the establishment of stable covalent linkages between the protein and the chemically modified PDMS surface. The differences in Col1 binding in each case could be attributed to the nature of protein adhesion on the respective substrates. On the native substrates, Col1 adsorption is random, weak and especially dominated by charge based /electrostatic interactions. Hence a substantial loss of proteins from the 
PDMS surface after day 7 was seen. On the surface of APTES functionalized PDMS surfaces, the protein adsorption is relatively stronger than plain PDMS+Col1 on all days which could be mainly attributed to the active interaction of Coll with the ionically active layers of APTES molecules. The APTES+GA substrates showed the most stable (high) adhesion of Coll compared the rest of the substrates due to high probability of covalent interactions of amine $\left(-\mathrm{NH}_{2}\right)$ side chains of Col1 with the carboxyl (-COOH) groups of GA. On all three substrates, a higher degree of surface bound protein at day 7 as compared to day 1 was observed. This is due to a relatively lower degree of initial Col1 attachment through weaker physical interactions during day 1 . Since the kinetics of both protein adsorption is expected as a gradual exponential increase in protein attachment, a slow and progressive increase in the strength of ionic/covalent linkages and stabilization of protein attachment on the substrates was observed on day 7 . However, after day 14 , the amount of surface protein declined as compared to day 7 , which could be due to the protein degradation under incubation conditions $\left(37^{\circ} \mathrm{C}\right.$ and $\left.5 \% \mathrm{CO}_{2}\right)$. Nevertheless, chemically modified PDMS surface showed an improved surface protein immobilization and stability compared to the native PDMS. 


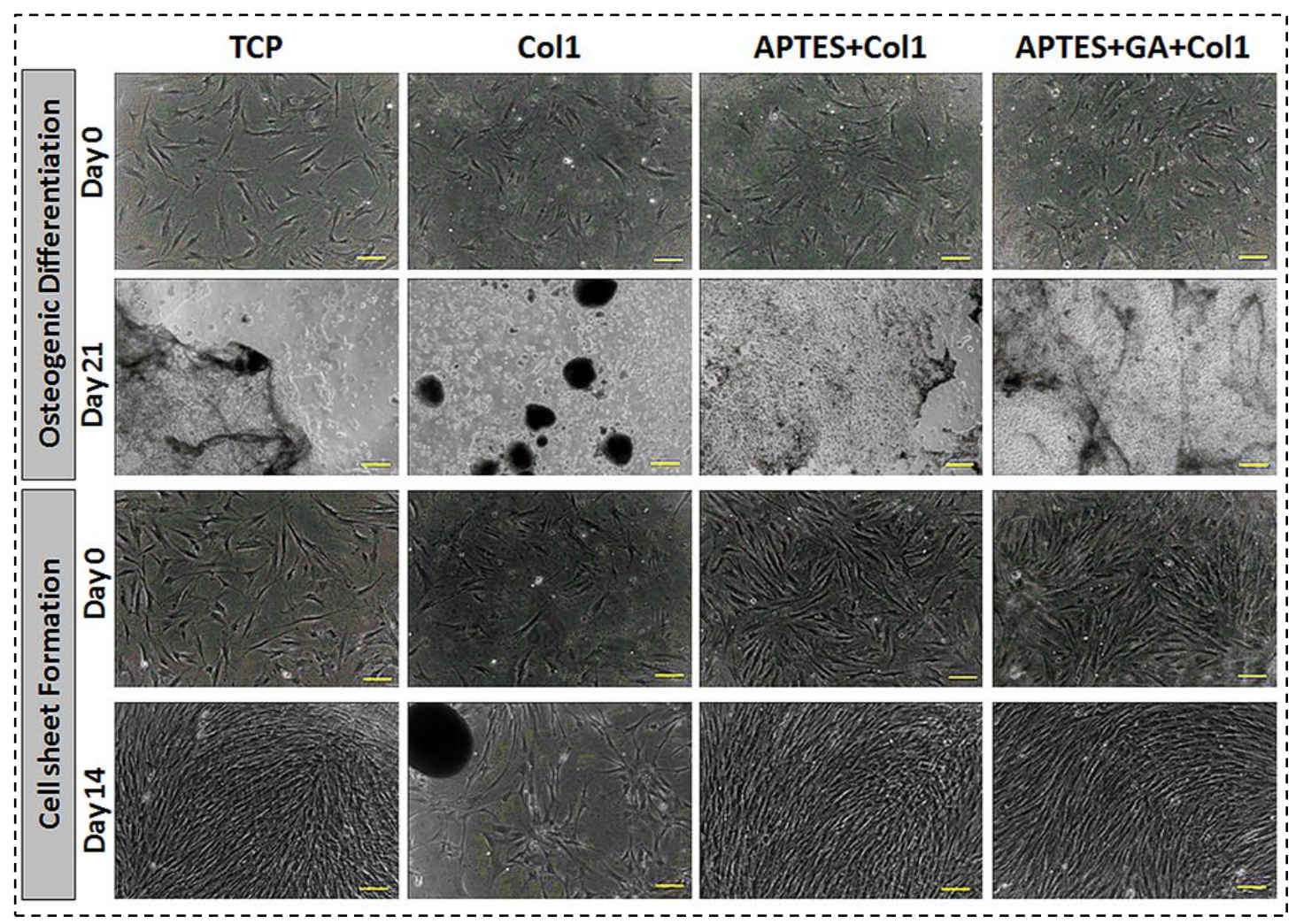

Figure 4-13: Investigation on osteogenic differentiation of hMSCs and hMSC sheet formation on unmodified and chemically modified PDMS with Col1 immobilization. TCP surface: positive control. (Scale bar: 200 um)

The use of hMSCs in osteogenic differentiation and cell sheet engineering is often investigated in vitro to improve hMSC-mediated tissue regeneration. hMSCs were cultured under two different culture media to induce osteogenesis and cell sheet formation, and their cell adhesion was monitored with a phase contrast microscope (Figure 4-13). Initial cell seeding after $6 \mathrm{~h}$ was observed on all surfaces (day 0). However, total cell dissociation was observed as clumps of cell aggregation (dark spots in the 2nd column) on unmodified PDMS surfaces although with Col1 coating during both osteogenic differentiation (day 21) and cell sheet formation (day 14). The instability in cell adhesion on these native PDMS surfaces could be due to the hydrophobic recovery over time (Figure 4-10) and the weakly bound matrix proteins (Figure 4-12). In contrast, on the silanized surfaces with APTES and Coll coating, minimal or no cell dissociation (Figure 
4-13) were observed in either osteogenic differentiation or cell sheet formation. It was known that higher roughness profile could provide a higher surface area for cell-substrate interaction and thus encourage cell adhesion and proliferation [22-27]. Although the surface roughness was rather low on the chemically modified PDMS with APTES+Col1 (Figure 4-11), the stability of confluent cell layer adhesion was significantly improved.

In addition, PDMS surface modified with APTES+GA+Col1 exhibited a raised roughness profile (Figure 4-11), improved surface wettability Figure 4-10), increased protein adsorption (Figure 4-12), and thus further stabilized the cell adhesion at confluence with no observable incidents of cell dissociation (Figure 4-13). As shown in our previous study, the surface silanization could enhance the proliferation of individual hMSCs on PDMS substrate [17]. Compared to the conventional TCP, chemically modified PDMS surfaces provided a much more stable platform for long-term cell adhesion and proliferation.

Dowling et.al [35] showed that a hydrophilic contact angle range of $\sim 60-70^{\circ}$ on plasma modified polystyrene resulted in enhanced adhesion of Osteosarcoma cells. Arima et.al [36] highlighted that along with surface wettability, the surface functional group also affects cell adhesion, with the highest density of adherent cells on the amine $\left(-\mathrm{NH}_{2}\right)$ terminated surface treatment. He et.al [37] showed that effective collagen self-assembly depends on underlying active functional groups which further determines healthy MSC adhesion and spreading [36]. In our study as well, although the TCP (control) surfaces positively influenced cell adhesion, the functionally active APTES+Col1 or APTES+GA+Col1 substrates supported overall cellular expressions such as stable cell sheet formation and enhanced osteogenic gene expression. Since, the chemically modification resulted in improvement in surface wetting, functional reactivity and protein stability compared to unmodified plain PDMS, a possibility of combined influences of these parameters could be seen to have affected the overall behavior of hMSCs rather than an isolated influence of a single or specific factor. 
The hMSC-substrate interaction is crucial for osteogenic differentiation, and it is therefore important to evaluate the effect of specific surface chemistry for its suitability in long-term cell culture. For instance, numerous studies demonstrated that enhanced osteogenic differentiation could be achieved by increasing the roughness profile of a substrate, reducing surface hydrophobicity [28], providing functional group that benefit surface protein attachment [29-30] and subjecting hMSCs to nano-topographical cues [31].

Chemically modified PDMS surfaces with APTES \pm GA+Col1 present a hydrophilic surface (Figure 4-10) with functional groups that facilitate better attachment of Col1 protein (Figure 4-12). As Col1 promotes the proliferation [29] and osteogenesis of hMSCs [29-30], the increased Col1 immobilization on APTES \pm GA modified PDMS surfaces could enhance osteogenic differentiation. Further, nano-ridge formation after treatment of APTES+GA+Col1 provided nanotopological cues (Figure 4-11) that could possibly direct hMSC differentiation towards osteogenic lineage [31]. For further verification, osteogenic markers and bio-mineralization of osteogenic hMSCs were assessed. 


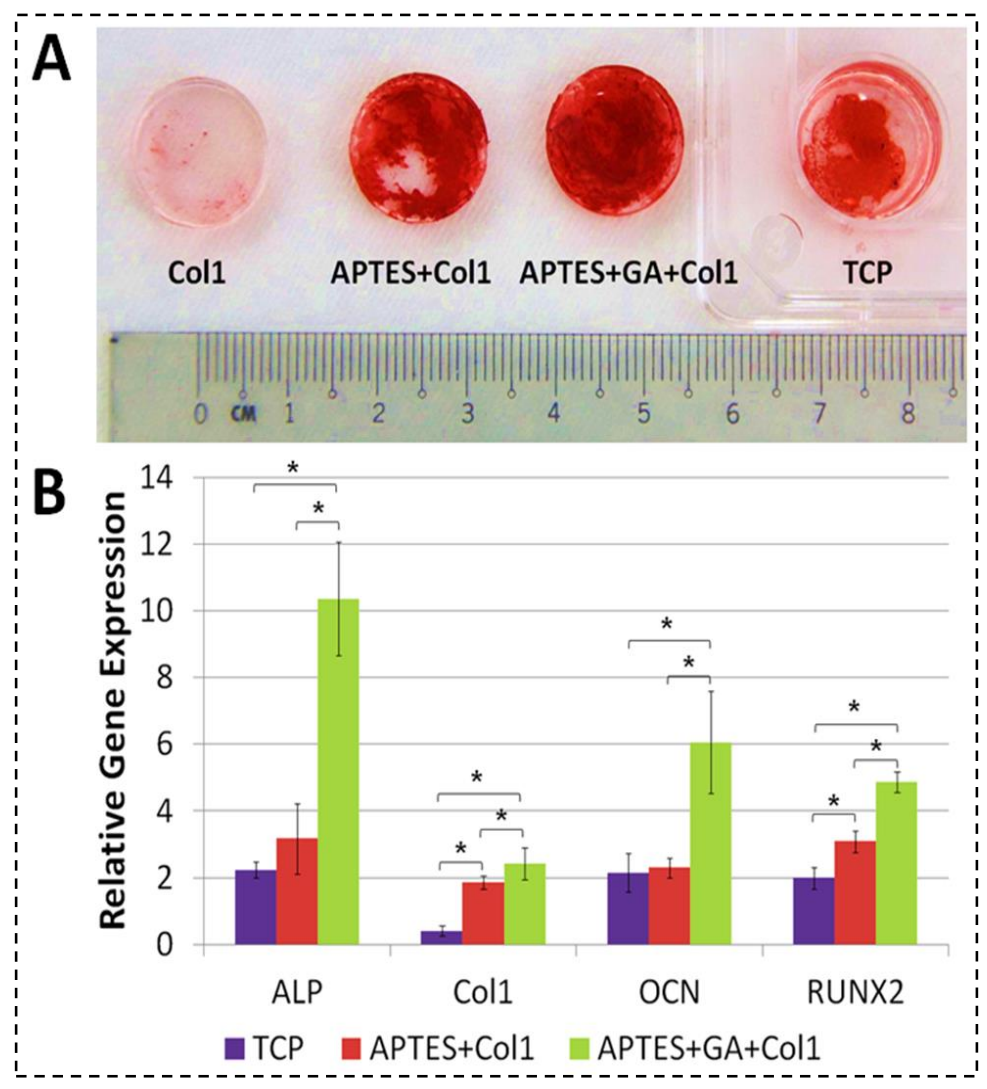

Figure 4-14: (A) Assessment of bio-mineralization on unmodified and chemically modified PDMS substrates with Col1 immobilization revealed by Alizarin red staining. TCP surface is used as control. (B) Expression of osteogenic gene biomarkers on unmodified and chemically modified PDMS substrates with Col1 immobilization. The data are shown as mean \pm SD. *p value of $<0.05$ between two groups under comparison.

The osteogenic lineage formation was demonstrated with Alizarin red staining, whereby the presence of brick-red nodules revealed the mineralization deposition during osteogenic differentiation (Figure 4-14A). Unmodified PDMS surfaces showed minimal staining while intense staining was observed on both chemically modified surfaces. Meanwhile, the osteogenic lineage on TCP surface showed a weaker staining as compared to the modified PDMS surfaces. These results indicated that silanized PDMS surfaces were capable of enhancing mineralization than the conventional TCP surfaces (Figure 4-14 A) yet stabilizing the hMSC adhesion. As common osteogenic biomarkers, alkaline phosphatase (ALP), osteocalcin (OCN), Col1 and Runx2 were used to quantitatively assess the effects of chemical modifications on osteogenesis. The data 
showed that osteogenic hMSCs on PDMS surfaces modified with APTES+GA+Col1 exhibited significantly higher expression of all four osteogenic biomarkers as compared to the other two surfaces, including TCP (Figure 4-14 B). Gene expression could not be evaluated for the cells grown on unmodified PDMS owing to cell aggregation and dissociation at the point of analysis (Figure 4-13).

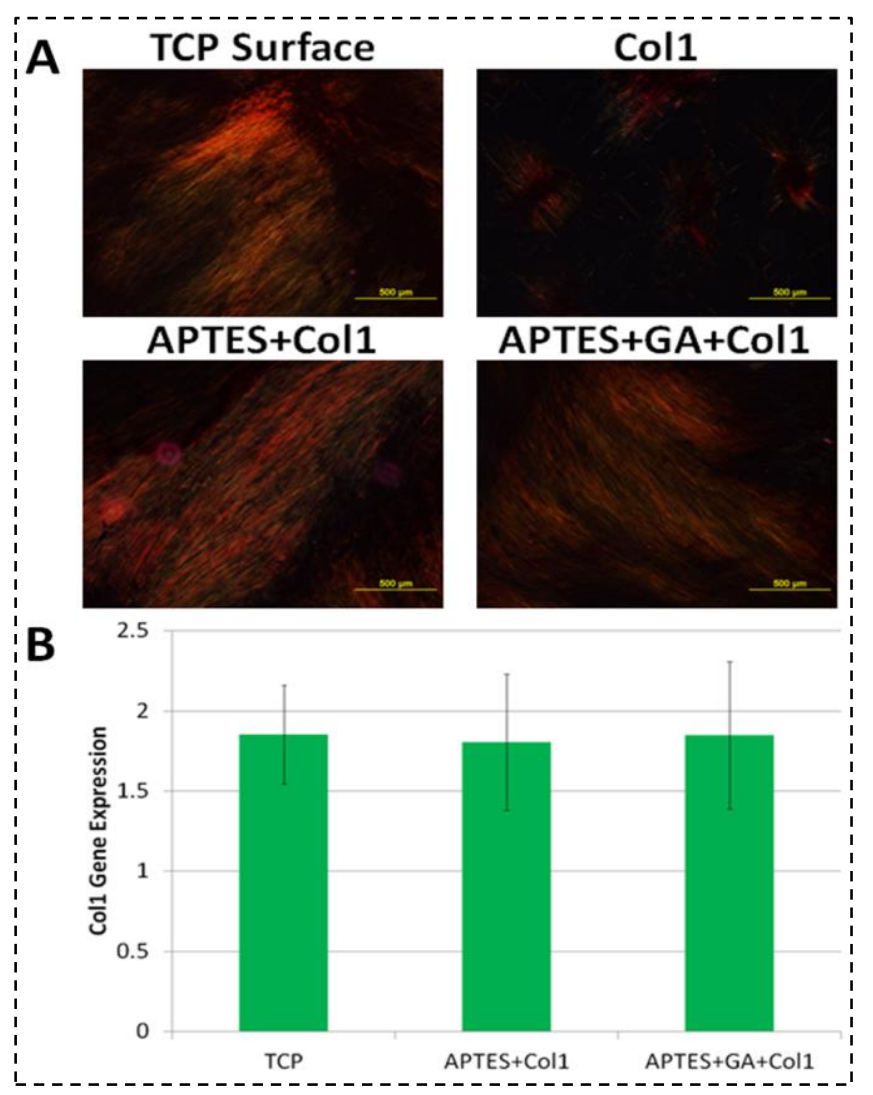

Figure 4-15: (A) Collagen formation on unmodified and chemically modified PDMS surfaces as revealed by Picro-Sirius red staining under polarizing microscope. TCP surface is used as control. (B) Col1 gene expression of hMSC sheet after 2 weeks of culture on unmodified and chemically modified PDMS surfaces.

The formation of an intact cell sheet relies on cell-cell interaction and the presence of active ECM constituents, which supply the mechanical and chemical signals to mediate the cellular physiology and provide physical support for tissue development [32]. In this study, the hMSC cell sheet formation was characterized by Picro Sirus Red staining (Figure 4-15 A). It was observed that 
collagen fibers could not be formed on unmodified PDMS surface, which could be attributed to the hMSC dissociation and aggregation after 2 weeks of culture. On the other hand, the stability of hMSC adhesion on the silanized PDMS surfaces allowed the formation of cell sheet as revealed by the prominent and bright red images under the polarizing microscope. The presence of collagen fibers was further verified by the quantitative analysis of Coll gene expression, which showed similar profiles on the silanized PDMS surfaces as compared to TCP (Figure 4-15B).

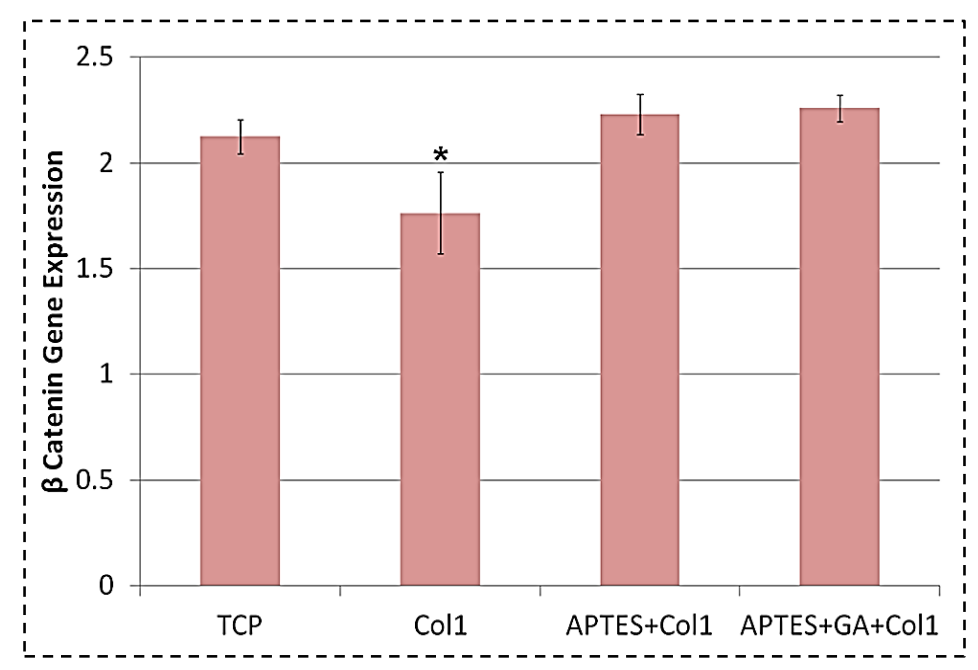

Figure 4-16: $\beta$-Catenin gene biomarker expression of 1-week-old hMSC sheet on unmodified and chemically modified PDMS substrates with Col1 immobilization. The data are shown as the mean \pm SD. *p value of $<0.05$ as compared to any groups.

$\beta$-Catenin is a protein within the cells that is responsible for the regulation of cell growth and cellcell adhesion [33]. Gene expression of $\beta$-Catenin was evaluated during the early stage of hMSC sheet formation (1 week) where minimal cell dissociation was observed on unmodified PDMS surface, making it possible to evaluate the gene expression of $\beta$-Catenin. Compared to the unmodified PDMS, a significant increase in the gene expression of $\beta$-Catenin on silanized PDMS surfaces (Figure 4-16) may eventually lead to higher protein level of $\beta$-Catenin, which could enhance the cell-cell adhesion to maintain the integrity of formed hMSC sheet. While compared with TCP surfaces, hMSC sheet formation was achievable on chemically modified PDMS surfaces without deterioration in Coll and $\beta$-Catenin expression. However, the subcellular 
localization of $\beta$-Catenin under the effect of surface silanization is still unclear in this study. It is well known that $\beta$-catenin plays a key role in cell adhesion and proliferation, and is regulated by multiple signaling pathways during stem cell development and tumorigenesis [34]. Further studies on $\beta$-Catenin localization in hMSCs would be helpful to understand how extracellular microenvironment affects stem cell biochemical signaling pathways through cell-surface interaction.

In summary, silanization with $\mathrm{APTES} \pm \mathrm{GA}$ changes the surface chemistry of PDMS, which becomes more hydrophilic thereby enhancing Col1 protein attachment. Although roughness profile varies between the two types of silanized surfaces, the interrelation with other surface properties further improved the stability of the confluence cell adhesion while maintaining both potency of the hMSCs and cell sheet formation.

\subsubsection{Conclusion}

Long-term cellular studies in PDMS-based microfluidic analytical devices are usually limited due to the inherent hydrophobicity and poor bio-affinity of the native PDMS surface. This study has shown that silanization by $\mathrm{APTES} \pm \mathrm{GA}$ cross-linking can profoundly change PDMS surface properties, including reduction of hydrophobicity, increased protein immobilization, and variation of nano-topography. The synergetic effect of these changes can be utilized to support the stability of confluent hMSC culture, while retaining their osteogenic potency and supporting cell sheet formation. We believe that this surface functioning strategy can benefit future studies using PDMS micro-devices for long-term cell development on a chip. 


\section{References}

[1] A. L. Paguirigan, D. J. Beebe, Bioessays, 2008, 30, 811.

[2] J. M. K Ng, I. Gitlin, A. D. Stroock, G. M. Whitesides, Electrophoresis, 2002, 23, 34613473.

[3] S. K. Sia, G. M. Whitesides, Electrophoresis, 2003, 24, 3563-3576.

[4] J. N. Lee, X. Jiang, D. Ryan, G. M. Whitesides, Langmuir, 2004, 20, 11684-11691.

[5] D. Fuard, T. T. Chevolleau, S. Decossas, P. Tracqui; P. Schiavone, Microelectron. Eng., 2008, 85, 1289-1293.

[6] J. C. McDonald, D. C. Duffy, J. R. Anderson, D. T. Chiu, H. Wu, O. J. A. Schueller, G. M. Whitesides, Electrophoresis, 2000, 21, 27-40.

[7] D.C. Duffy, J.C.McDonald, J. A. Schueller, G. M. Whitesides, Anal. Chem.,1998,70, 4974-4984.

[8] D. Bodas, C. Khan-Malek, Sens. Actuators B, 2007, 123, 368-373.

[9] H. Hillborg, U. W. Gedde, Polymer, 1998, 39, 1991-1998.

[10] A.J. Engler, S. Sen, H. L. Sweeney, D. E. Discher DE, Cell, 2006, 126, 677-689.

[11] M. Kassem, Ann NY Acad Sci, 2006, 1067, 436-442.

[12] J. Protivínský, M. Appleford, J. Strnad, A. Helebrant, J. L. Ong, Int J Oral Maxillofac Implants, 2007, 22, 542-550.

[13] S. Lavenus, J.C. Ricquier, G. Louarn, P. Layrolle, Nanomedicine, 2010, 5, 937-947.

[14] H. Yuan, W. M. Mullett, J. Pawliszyn, Analyst, 2001, 126, 1456-1461.

[15] C. M. Halliwell, A. E. G. Cass, Anal. Chem., 2001, 73, 2476-2483.

[16] Z. H. Wang, G. J. Jin, Immunol. Methods, 2004, 285, 237-243.

[17] S. Kuddannaya, Y. J. Chuah, MHA. Lee, N. V. Menon, Y. Kang, Y. Zhang, ACS Applied Materials \& Interfaces, 2013, 5, 9777-9784.

[18] M. Ishii, R. Shibata, Y. Numaguchi, T. Kito, H. Suzuki, K. Shimizu, A. Ito, H. Honda, T. Murohara, Arterioscler Thromb Vasc Biol, 2011, 31, 2210-2215.

[19] Y. Qi, Y. Du, W. Li, T. Zhao, W. Yan, Knee Surg Sports Traumatol Arthrosc, 2014, 22, 1424-33.

[20] Y. Qi, T. Zhao, W. Yan, K. Xu, Z. Shi, J. Wang, Cytotherapy, 2013, 15, 44-56.

[21] M. Augustin, M. A. A. Mahar, P. Lakkisto, I. Tikkanen, A. Vento, T. Pätilä, A. Harjula, J Tissue Eng Regen Med, 2013, 7, 742-750.

[22] E. Biazar et.al, International Journal of Nanomedicine, 2011, 6, 631-639.

[23] S. Patntirapong, W. Singhatanadgit, P. Meesap, T. Theerathanagorn, M. Toso, W. Janvikul, J Biomed Mater Res Part A, 2014, [Epub ahead of print].

[24] A.F. Recum, C. E. Shannon, C. E. Cannon, K. J. Long, T. G. Kooten, J. Meyle, Tissue Eng, 1996, 2, 241-253.

[25] K. Hatano, H. Inoue, T. Kojo, T. Matsunaga, T. Tsujisawa, C. Uchiyama, Y. Uchida, Bone, 1999, 25, 439-445.

[26] T.W. Chung, D. Z. Liu, S. Y. Wang, S. S. Wang, Biomaterials, 2003, 24, 4655-4661.

[27] K. Kieswetter, Z. Schwartz, D. D. Dean, B. D. Boyan, Crit Rec Oral Bio Med, 1996, 7, 329-345.

[28] S. Anil, P.S. Anand, H. Alghamdi, J. A. Jansen, 2011, doi: 10.5772/16475.

[29] K. S. Tsai, S. Y. Kao, C. Y. Wang, Y. J. Wang, J. P. Wang, S. C. Hung, J Biomed Mater Res A, 2010, 94, 673-82.

[30] S. Mathews, R. Bhondea, S. Totey, Biochem Biophys Res Commun, 2011, 414, 270-276.

[31] J. Kim, H. N. Kim, K. T. Lim, Y. Kim, H. Seonwoo, S. H. Park, H. J. Lim, D. H. Kim, K. Y. Suh, P. H. Choung, Y. H. Choung, J. H. Chung, Sci Rep, 2013, 3, 3552.

[32] C. M. Nelson, M. J. Bissell, Annu Rev Cell Dev Biol, 2006, 22, 287-309

[33] F. H. Brembeck, M. Rosário, W. Birchmeier, Curr Opin Genet Dev, 2006, 16, 51-59.

[34] N. Barker, M. van den Born, Methods Mol Biol, 2008, 468, 91-98. 
[35] Dowling, D.P., et al., Journal of Biomaterials Applications, 2011. 26(3): p. 327-347

[36] Y. Arima., H. Iwata, Biomaterials, 2007, 28(20), p. 3074-3082

[37] J. He, Y. Su, T. Huang, B. Jiang, F. Wu, Z. Gu, Colloids.Surf Biointer., 2014, 116, p. 303- 


\subsection{Effect of silane type, structure and functionality on hMSC fates: miniaturized model to evaluate cell-surface chemical interactions on PDMS microchip}

\subsubsection{Background}

Cell behaviors are profoundly influenced by the biochemical cues in their immediate native microenvironments [1,2]. Mammalian cell fates in vitro can be regulated by diverse chemical signals stimulated by the supporting ligands [3,4], biomolecules [5-7] or synthetic functional molecules $[8,9]$ in the cell vicinity. Past studies indicated the physiological relevance of biochemical microenvironments in diverse spatio-temporal combinations which could be designed by micropatterning techniques [10-12]. The reactivity of the surface towards proteins or cells is highly dependent on the dominant surface functional groups which determine the charge $[13,14]$, wettability $[13,15]$ and functional specificity of surfaces. In this regard, several functionally specific molecules belonging to the organo-silane class have been increasingly exploited for their role in forming reactive self-assembled monolayers (SAMs) [12, 16]. Organosilanes vary in structure and reactive functional group, and thus display various polymerization dynamics and molecular specificity which has been highly exploited for controlled functionalization of biomolecules/ligands in high performance biosensor/diagnostic applications [16-20]. However, only few studies have shown the detailed role of these molecules in direct regulation of various mammalian cell behaviors mostly on glass and silicon substrates [12, 21-23]. Past studies in this regard are limited to glass, silica, PMMA and gold based substrates which limit the application of silanization towards advanced and complex cell based studies. The easy tunability and fabrication of PDMS structures provided significant advantages for rapid prototyping of cell microenvironments applicable in cell behavioral investigations $[11,24-26]$. Recently, we demonstrated APTES silanization followed by glutaraldehyde (GA) cross-linking of ECM proteins on PDMS substrates which resulted in a drastic decrease in PDMS inherent 
hydrophobicity and enhanced surface biocompatibility [27]. The resulting substrate enabled significantly enhanced adhesion, long-term viability and potency of human mesenchymal stem cells (hMSCs) compared to the unmodified substrates [27, 28]. Since functional moieties can directly interact with trans-membranal proteins on cells or serum proteins in culture environments, direct interaction of cells on silanized PDMS can also play a regulatory role in mediating focal adhesion and in directing subsequent cell behaviors such as proliferation, migration and differentiation $[29,30]$. Although studies have shown the role of functional group in directing cell morphology and behaviors [30-34], little is known about their exact role in promoting long term adhesion, proliferation and differentiation of multipotent cells such as the hMSCs. Since silanization offers variations in surface distribution, polymerization, reactivity and specificity of exposed functional groups, its direct influence on the hMSC adhesion, cell sheet formation and potency can be investigated. However traditional silanization based covalent ECM protein binding on PDMS substrates are time consuming, involves laborious protocols and is prone to batch variations. Hence there is a need to develop efficient yet highly functional and easily tunable silanization strategies which can be conveniently adapted for high throughput cell based studies or cell investigations on microfluidic/micropatterned platforms. 


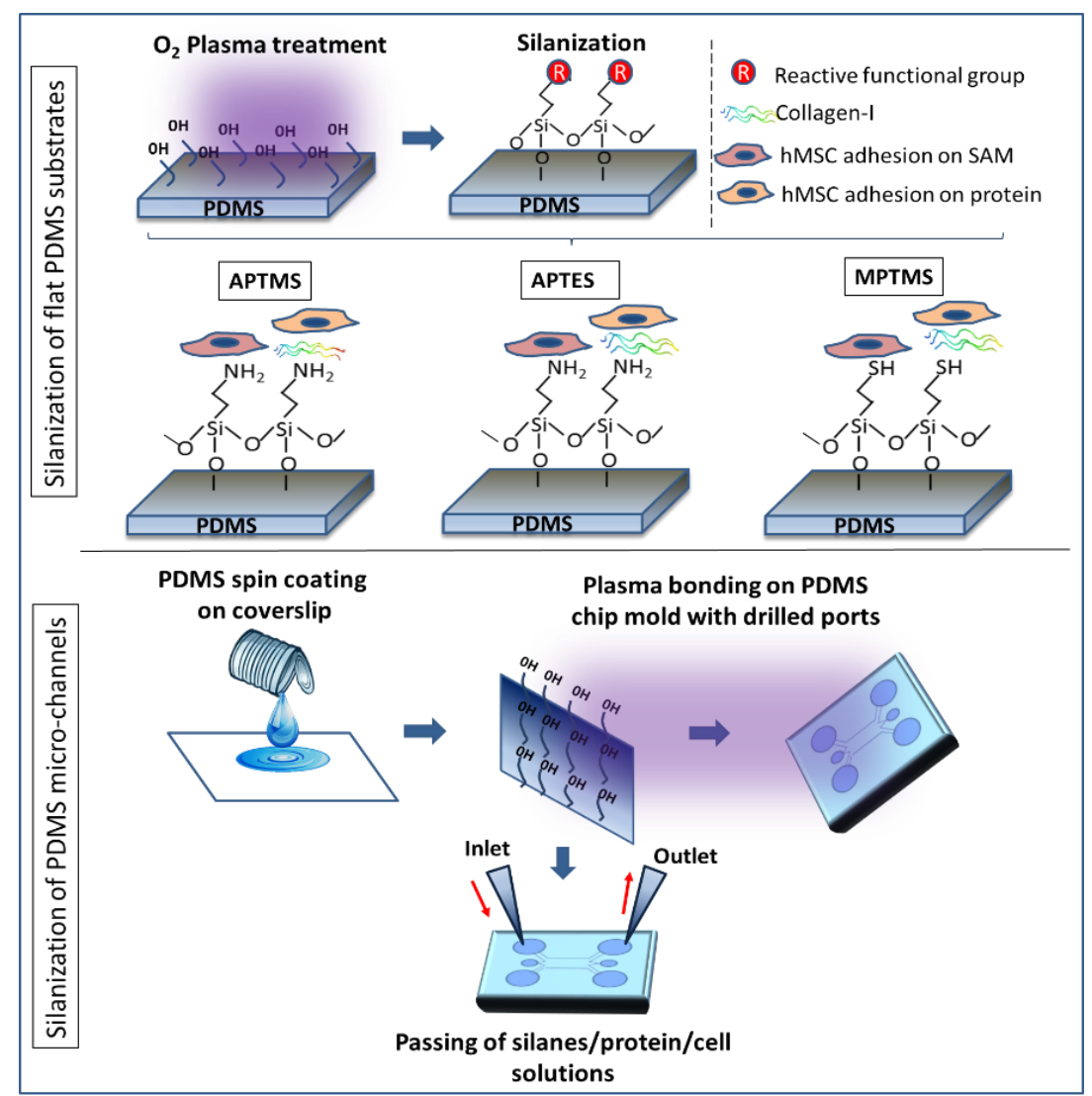

Figure 4-17: (Top) Schematic representation of surface silanization and collagen immobilization followed by hMSC culture on flat substrates. (Below) Silanization and hMSC culture within PDMS micro-channels

To address these issues, in this study we discuss the direct impact of variations in silane molecular structure and surface reactive functional group in determining hMSC cell fates (Figure 4-17). To test our hypothesis, we employed silanization of PDMS by oxygen plasma treatment and subsequent treatment with three commonly studied silanes varying in molecular structure, SAM layer formation kinetics and the functionality of exposed reactive group. APTES is a commonly used silane owing to the simplicity in silanization conditions and presence of reactive $\left(-\mathrm{NH}_{2}\right)$ groups which can react with biomolecules or cell surface proteins through active ionic interactions [35-38]. To know the effect of molecular structure in forming stable SAM layers as well as to study their eventual interaction with hMSCs, we used a similar amine terminated silane (3- 
Aminopropyl) trimethoxysilane (APTMS) consisting of tri-methoxy group $\left(-\mathrm{OCH}_{3}\right)$ in place of the tri-ethoxy group $\left(-\mathrm{OC}_{2} \mathrm{H}_{5}\right)$ of the APTES. In past APTMS has also been used for highly specific amine $\left(-\mathrm{NH}_{2}\right)$ group functionalization especially on glass and gold substrates due to amine reactivity and high tendency of forming positively charged SAMs [39, 40]. Additionally, to assess the role of thiol $(-\mathrm{SH})$ based linkages against the amine based $\left(-\mathrm{NH}_{2}\right)$ based cross-linking, we employed silanization with a thiol group terminated (3-Mercaptopropyl) trimethoxysilane (MPTMS). The use of MPTMS has been widely employed in highly sensitive detections and functionalization studies [41, 42]. However, since past studies have presented only a limited understanding of thiol based strategies for stable adhesion of mammalian cells, and further due to higher bond stability of thiol linkages, the efficiency of MPTMS based silanization was compared with amine reactive silanes for their potential role in mesenchymal fate direction. The amine ($\mathrm{NH}_{2}$ ) and thiol (-SH) based silanes have shown the probability to form either covalent like linkages or ionic interactions with proteins through formation of stable amide (-CONH) and disulphide (-S-S) linkages respectively. In this study, we performed PDMS surface silanization by APTES, APTMS and MPTMS for collagen type 1 (Col1) ECM protein immobilization and evaluated the influence of silanization with/without the bio-functionalization of Col1.

The characteristic differences in the molecular structure and functionality of these silanes present diversity in polymerization kinetics, thickness of the SAM layer, cross-linking capacity and long term stability of the adhered SAM layers. Further the silanization could influence variable degree of PDMS surface hydrophobicity and roughness which are critical parameters that determine the quality of both ECM protein immobilization and cell adhesion [27, 43]. In our study, the APTES/APTMS/MPTMS based silanization were compared for ECM protein attachment and hMSC adhesion on PDMS substrates. We report a quick and easily implementable PDMS silanization strategy for long term investigation of hMSCs behaviors. A PDMS chip with $150 \mu \mathrm{m}$ height and comprising of three compartments separated from each other by arrays of rectangular pillars (cross-section $200 \mu \mathrm{m} \mathrm{X} 100 \mu \mathrm{m}$ ), which extends over $1 \mathrm{~cm}$ along the microchannels was 
designed. The width of the side channels and the central channels are $800 \mu \mathrm{m}$ and $500 \mu \mathrm{m}$ respectively. This design was originally designed to support co-culture of two cell types on the microchannels on extreme ends. In this work, we used the chip to test the biofunctionalization and hMSC viability in microfluidic environments since the design can facilitates cell penetration and distribution across a wide area. Hence the design is aimed to test the potential to form a functional cell sheet sandwiched within the silanized microchannels. Eventually the stable cell sheets formed within these channels were tested to undergo osteogenic differentiation on chip. The silanization not only improved surface cytocompatibility but also promoted significantly higher cell layer stability, biomineralization and enhanced expression of osteogenic biomarkers on both standard PDMS substrates and miniaturized (PDMS micro-chip) models. Our platform is aimed to propel further studies on stable, robust, easily adaptable and scalable models for systematic long-term study of cell behaviors in the context of directed differentiation and tissue development. 


\subsubsection{Results and discussion}

\subsubsection{Surface hydrophobicity, Roughness and protein retention}
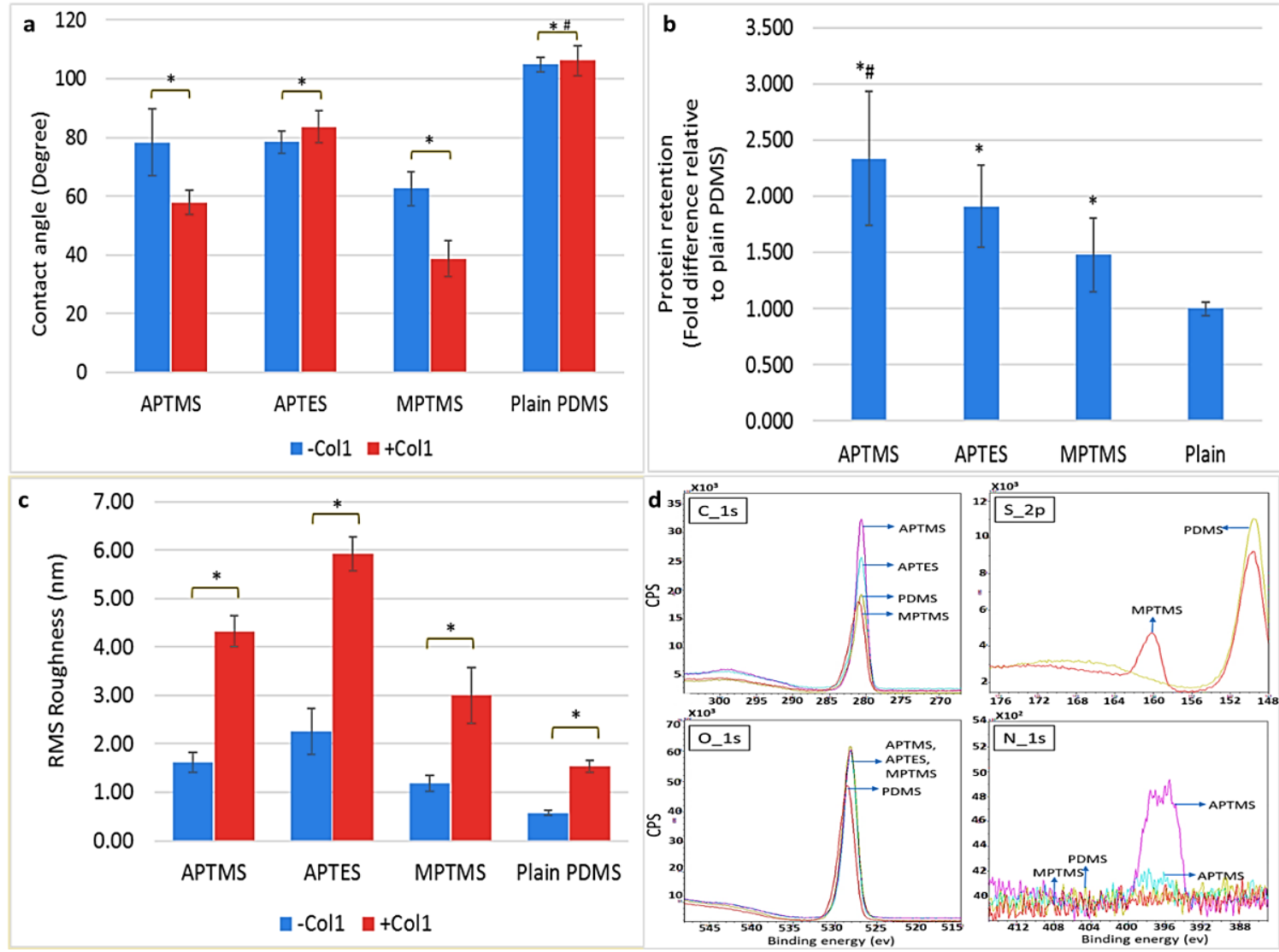

Figure 4-18: (a) Water contact angle measured on silanized substrates with/without col1 treatment (b)

Measurement of protein retention on silanized substrates (c) Root mean square (RMS) nano-scale surface roughness measurement by AFM (d) XPS analysis of silanized and unmodified (native PDMS) substrates

Variations in the surface energy changes induced due to silanization induce resultant changes in wettability/hydrophilicity of the surfaces. The relative degree of wettability was assessed by the contact angle measurements on both collagen coated and uncoated silanized substrates (Figure 4-18a). Although slight variations in surface wettability is seen among the silane groups, generally, a clearly notable decrease in surface hydrophobicity induced by silanization was 
observed due to the significant differences in the contact angles between the modified and unmodified substrates. In most cases, substrates without collagen showed higher wettability compared to the collagen treated substrates which indicate that the exposed functional groups have higher surface energy and reactivity towards aqueous solvents. However, all the silane substrates supported a nearly comparable contact angle upon collagen adsorption within the range of 40-60 [44]. Surface wettability in this contact angle range has been shown to be optimal for improved cell adhesion since both extreme wettability as well as high hydrophobicity can induce undesired and poor interaction with cells [44].

Since surface wettability induced by SAM layers of silane molecules can affect bio-adsorption, the degree of protein retention between the silanized and unmodified substrates were compared (Figure 4-18 b). Irrespective of the type of interacting functional group, the silanized substrates favored significantly higher protein retention compared to plain (unmodified) PDMS. A slight increase/decrease in the contact angles in +Col1 substrates as well as the variations in the surface retention of collagen on various silane layers could be attributed to the variations in the conformation of collagen which could be influenced by the underlying functional group [45, 46]. Among the silanized substrates, significantly high protein retention was observed on the APTMS modified substrates. Although APTMS have functional similarity (-NH2) with APTES, there is structural dissimilarity due the predominant presence of methyl and ethyl groups respectively. Since APTMS silanization has higher probability of forming uniform SAM layers with higher polymerization rate, there is a higher probability of surface coverage and even distribution of net positive charges on SAM layers which can both readily and actively interact with the proteins. A good wettability was noted for MPTMS substrates relatively lower degree of protein adsorption was observed. Hence a variation in protein preferences to both functionality and silane structure or charge could be seen. Although a lower degree of initial protein adsorption was evident, due to the high probability of forming strong disulfide (-S-S-) like bridges with the contacting proteins, MPTMS layers are known to foster a stronger rather than higher anchorage of proteins. A 
possibility of direct covalent-linkage of the thiol group with cysteine residues on protein side chains are known to be advantageous over the - $\mathrm{NH} 2$ group interactions mediated by the lysines [47]. Measurement of nano-scale roughness revealed a significant increase in surface roughness because of silanization. In each group (Figure 4-18 c), immobilization of Col1 resulted in a further increased the roughness. Since nano-scale topographic changes can significantly influence focal adhesion, the effect of each silane in facilitating optimal cell attachment could be studied. Analysis of elemental composition revealed characteristic peaks depending upon the molecular feature of each silane (Figure 4-18d) which confirmed the successful adsorption of respective SAMs. 


\subsubsection{Cell morphology, initial adhesion and surface coverage (D14)}
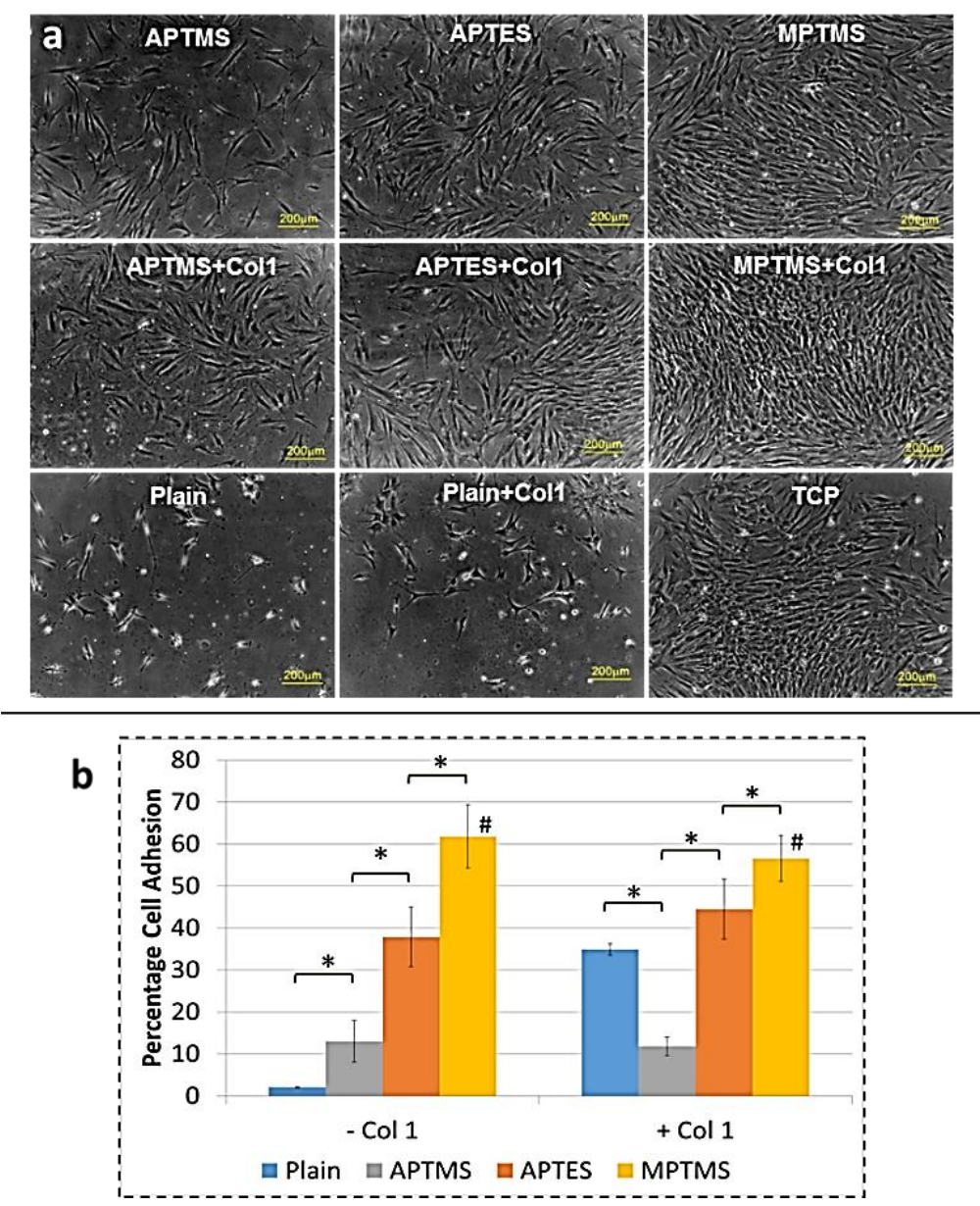

Figure 4-19: (a) Cell morphology and coverage (D1) on the silanized and unmodified substrates. (b) Initial cell (90 $\mathrm{min}$ ) cell adhesion on the modified/unmodified substrates as percentage of initial cells.

Compared to the unmodified (Plain) and the protein adsorbed (Plain+col1) substrates a distinguishably healthy hMSC adhesion and morphology could be observed on the silanized substrates (Figure 4-19). Silanization could not only primarily affect in forming a stronger initial cell adhesion but also promoted healthy morphology of cells. On the contrary, the weaker cell adhesion on the Plain and Plain+Coll substrates are characterized by irregularity in cell coverage as well as morphology (Figure 4-19a, row 3). Most cells in these substrates display clumped/rounded morphology which could be attributed to a weaker anchorage affected by poor focal adhesions. 
It is notable that the silanized substrates without Coll could form result in good cell coverage and anchorage. However, the presence of collagen on silanes substrates showed higher probability of uniform cell sheet formation which is desired for healthy differentiation. Among the silanes, the high cell and cell sheet formation was observed on MPTMS substrates, especially with Col1 modification. This trend indicates that although APTES and APTMS surfaces affected higher protein retention, the MPTMS surfaces affected highly favorable cell morphology and growth. Previous studies indicate that MPTMS are known to form thin but strong monolayers with high protein specificity. Between APTMS and APTES, relatively higher cell coverage with slightly higher cell to cell contact was observed on APTES surfaces compared to APTMS surfaces. This behavior could be linked with the relatively higher initial cell adhesion strength on the APTES surfaces (Figure 4-19 b). On the MPTMS surfaces (with/without collagen), the adhesion strength was significantly higher than all other substrates. The presence of protein (Col1) did not play a significant role in improving the initial attachment strength. However, the cell preference for initial attachment showed a preference to the nature of silane layer with a lower adhesion on APTMS, followed by enhanced adhesion on APTES and MPTMS surfaces, irrespective of Col1

coating (Figure 4-19 b). Further, detailed view of the cell adhesion characteristics was determined by the f-actin cytoskeletal profiles of hMSCs which reveal the cell spreading profiles after initial cell attachment.

\subsubsection{Cell spreading area}

Since a highly-spread profile of hMSCs with multiple focal adhesion points indicates higher cell anchorage on the substrates, differences in the cell spreading area because of silanization are compared. 


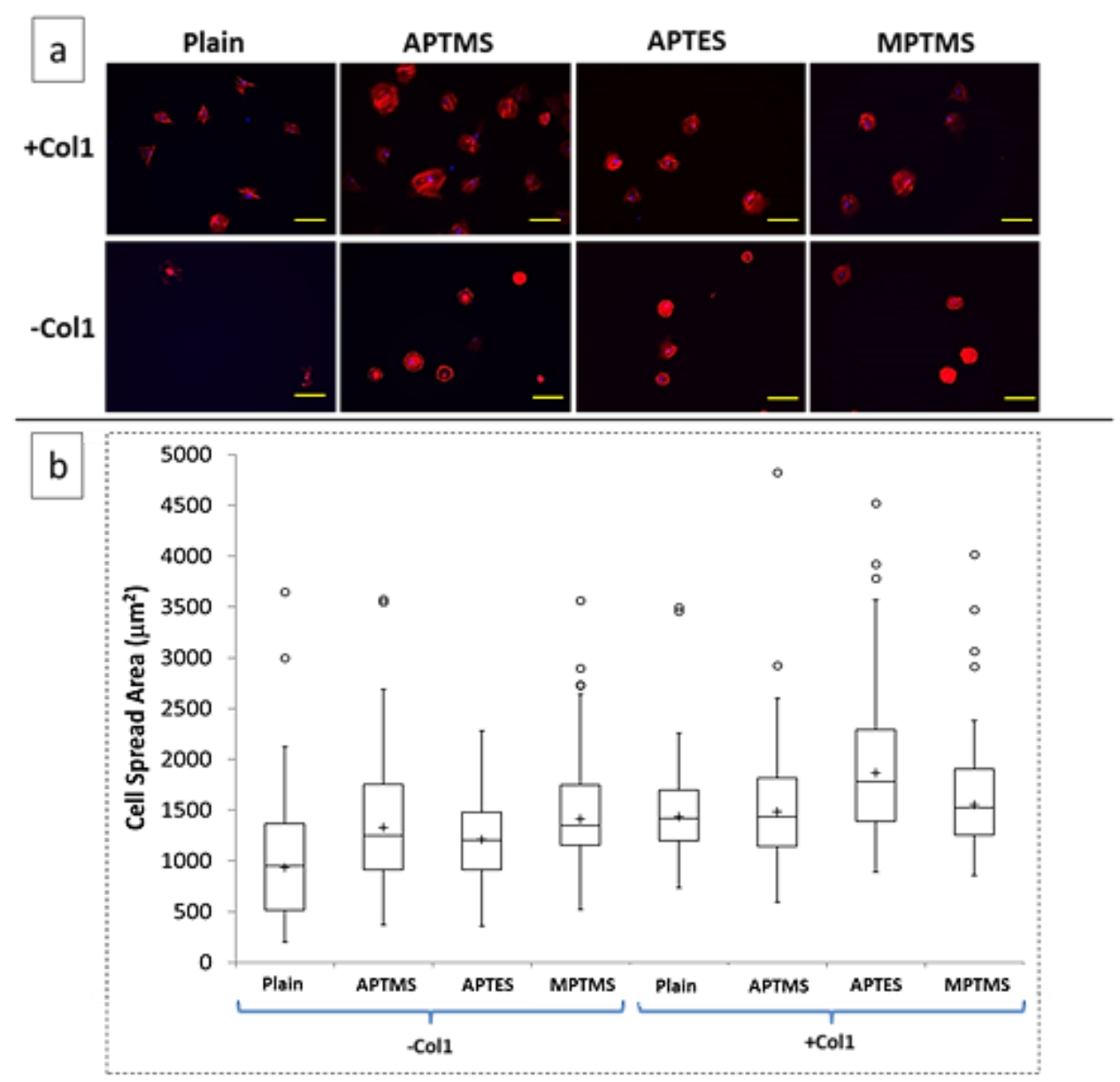

Figure 4-20: (a) F-actin profiles of hMSCs adhered on modified/unmodified PDMS substrates (b) Mean and median cell spread area on surfaces displayed in Box and Whiskers plots $(\mathrm{n}>30)$. Mean data are represented by $+{ }^{*} p$ value $<0.05$ between two groups. \#p value $<0.05$ as compared to collagen type 1 coated on the similar PDMS modified surfaces.

Higher spread areas were observed on silanized substrates compared to the native (unmodified) PDMS (Figure 4-20). The silanes substrate short enhanced spread area compared to unmodified substrates. It indicates that silanization induces enhanced propensity of formation of focal adhesion domains which result in highly spread cell profile. On the contrary, the lower degree of focal adhesion in unmodified substrates results in reduced cytoskeletal tension which negatively influences both cell morphology and activity [27]. Thus, the most cells assumed round morphology which was prone to dislodgement from the surface within 3-4 days of initial cell seeding. All three silanes showed a relatively similar level of cell spread area. However, with 
subsequent collagen coating on the solarized substrates, a slight increase in the cell area could be observed (Figure 4-20 b). Although the effect of col1 in influencing the strength of initial adhesion was not conclusive, silane+col1 substrates could positively affect cell morphology and spreading. This could be attributed to recognition of collagen as an immediate extracellular component by membrane integrins which can activate both actin polymerization as well as focal adhesion clustering. APTES+col1 substrates showed relatively higher spread area which could be result of beneficial reduced hydrophobicity, higher protein retention as well as the increased nano-scale roughness characteristics which can synergistically affect the strength of focal adhesion [27].

\subsubsection{Cell proliferation}

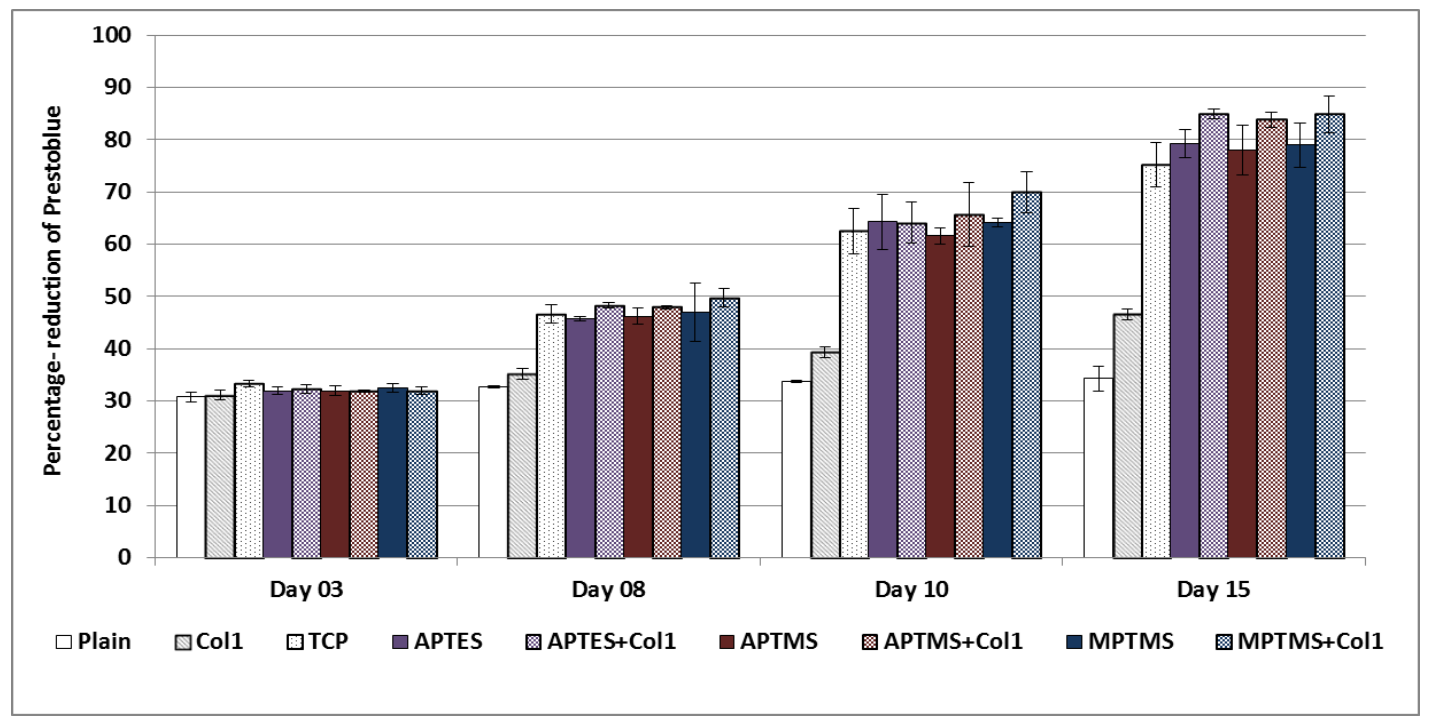

Figure 4-21: Proliferation of hMSCs on silanized and unmodified substrates measured on several intervals between Day 3 to Day 15 by the presto-blue assay.

The subsequent effect of favorable cell adhesion and morphology induced by silanization on long term viability and proliferation of hMSCs was assessed. Since lower degree of cell adhesion and viability is bound to affect cell proliferation, reduced level of proliferation was observed on both Plain and Plain+Col1 substrates (Figure 4-21). Col1 adsorption on plain PDMS could initially affect higher adhesion due to transient cell-substrate binding forces. However, on a longer run, a 
degradation of poorly adsorbed protein layers could be expected resulting in cell detachment from the unstable protein matrix. On the contrary, the silanized substrates resulted in an enhanced proliferation, with a clearly increasing trend with the progression of time. Coll immobilization especially with MPTMS and APTMS could affect in notably increased proliferation compared to other substrates. This shows that although APTES showed significant role in stabilizing the initial cell adhesion, relatively higher cell proliferation was eventually favored (post Day 01) on the Col1 immobilized APTMS substrates.

From these effects, the following key inferences could be drawn: (1) hMSCs sense the underlying silane characteristics which can affect their proliferation, by inducing surface changes as well as wettability (2) Silanization affected retention of active protein layer over long term culture conditions (3) The higher degree of cell adhesion and cell-cell contact (Figure 4-19) could positively influence cell division and metabolism (4) In the absence of Col1, the cell survival could be affected by synthetic silane environment.

\subsubsection{Adhesion and morphology within silanized micro-channels}

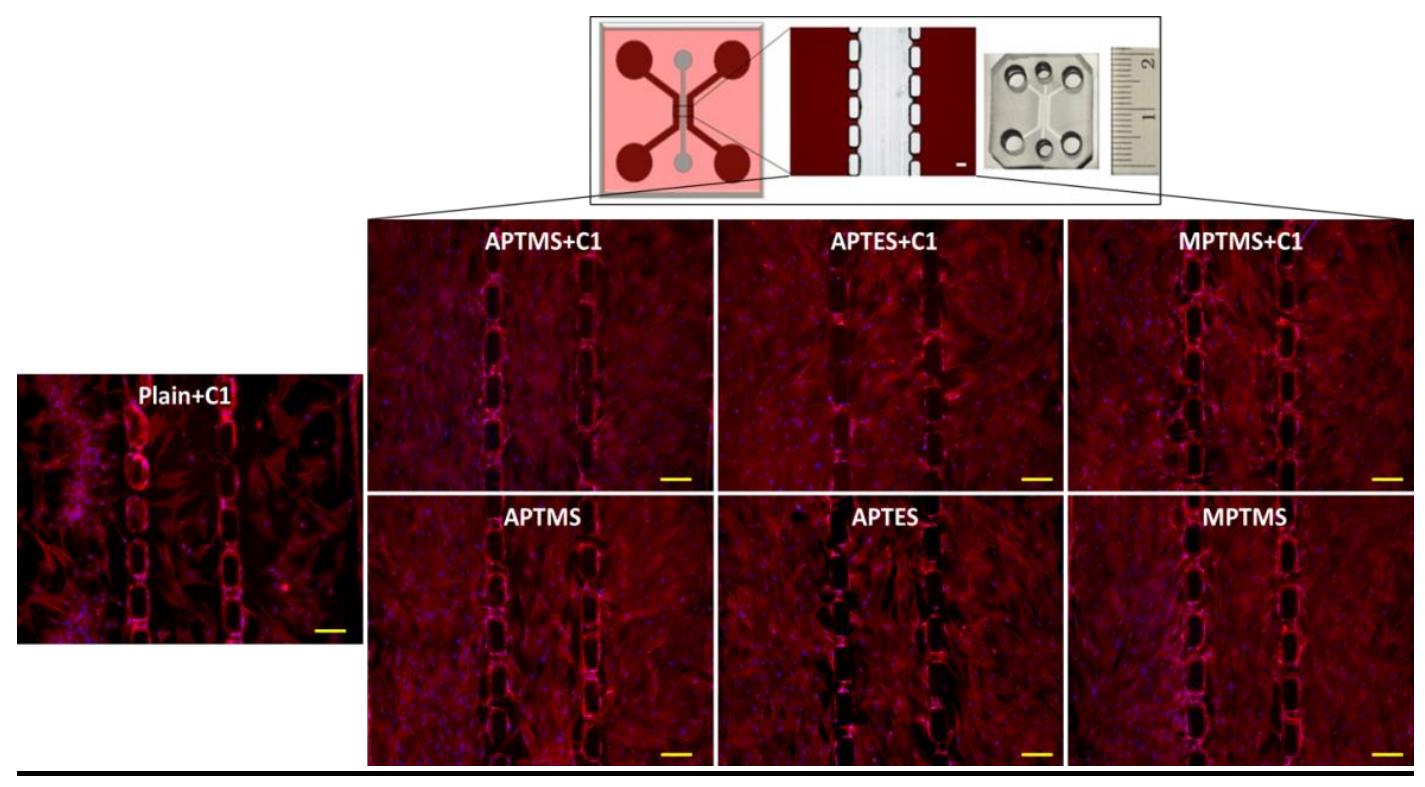

Figure 4-22: Morphology of adherent cell sheets within silanized PDMS micro-channels. Scale bar: $100 \mu \mathrm{m}$ 
Stable hMSC adhesion in the microchannel would be advantageous in long term investigation and regulation of cell fate within microfluidic models. The surface modification strategy could be conveniently implemented for the silanization of the PDMS micro-channels. Due to plasma induced reduction in microchannel hydrophobicity, a swift flow and reactivity of silane based aqueous solvents and subsequent protein and cell based solutions was achieved by maintaining simple hydrostatic flows. Hence a strong initial cell adhesion and hMSC growth was observed onchip (Figure 4-22). The inflow and outflow ports could be used to draw fresh medium and purge out the cell metabolites during the medium change procedures thereby promoting a healthy proliferation of hMSCs. Thus, the silanized channels enabled stable cell sheet formation with minimal cell dislodgement throughput span (Day 21) of the long-term culture as well as induction of osteogenic differentiation. Both APTES and APTMS modified micro-channels showed similar consistency of cell sheets with relatively higher cell-cell contact. 


\subsubsection{Bio-mineralization and osteogenic biomarker expression}

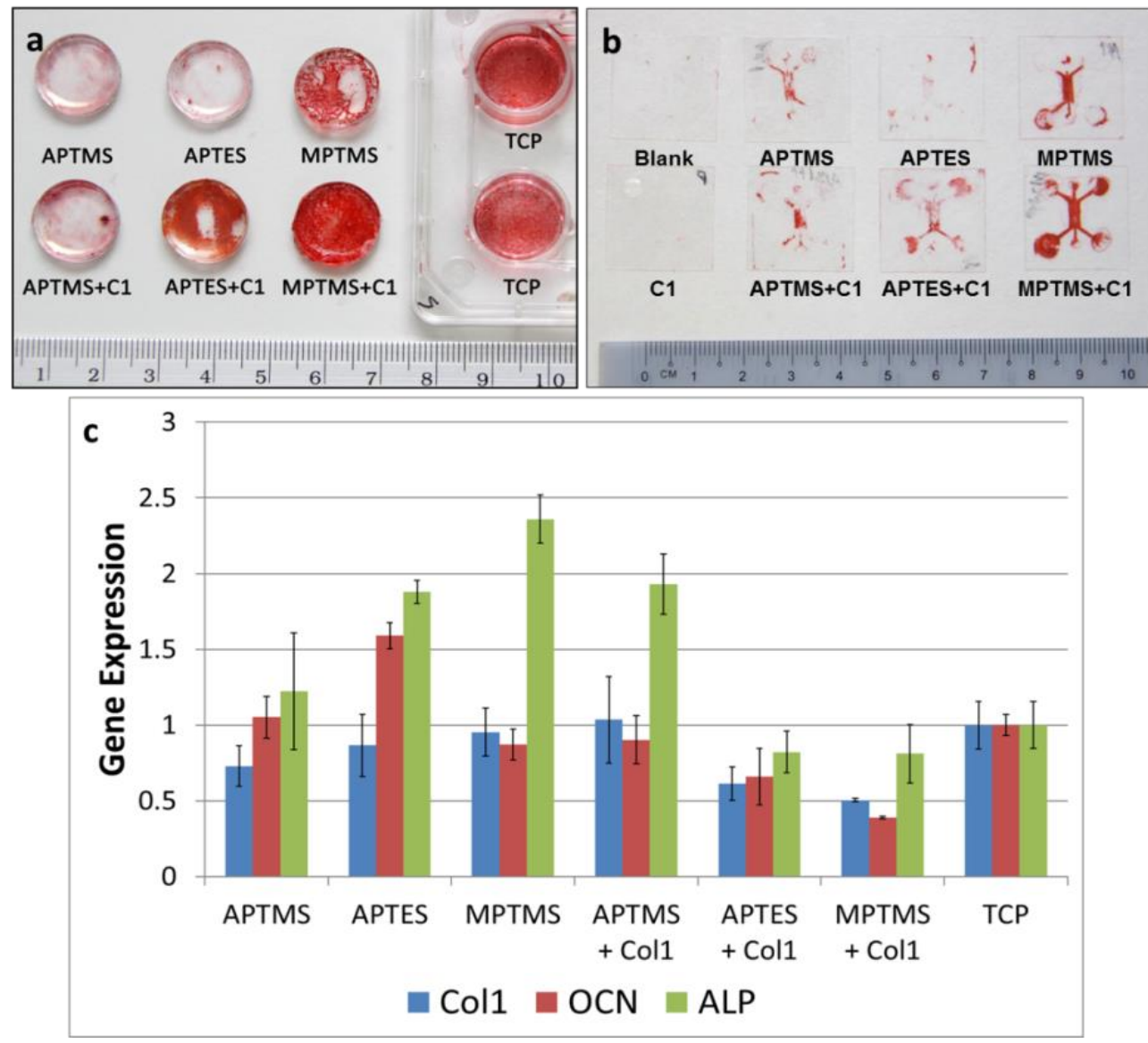

Figure 4-23: Assessment of calcium bio-mineralization following the osteogenic differentiation of hMSCs on silanized and unmodified PDMS substrates by calcium specific alizarin red staining (Day 21). (b) Expression of osteogenic genes (Day 21) on silanized substrates as a fold ratio of gene expression on standard TCP.

Since a poor cell adhesion was noted on the Plain PDMS substrates (with/without coll adsorption), these substrates were deemed unsuitable for both bio-mineralization and estimation of osteogenic gene expression. Due to the stable hMSC cell sheet formation was observed within the PDMS micro-channels, the system could enable induction of osteogenesis over a 21 day period and subsequent study of bio-mineralization within the microfluidic environment. Higher biomineralization was observed on both standard (flat) silanized substrates as well as the PDMS chip (Figure 4-23 a, b) with APTES and MPTMS modification especially with Col1 immobilization. A further accurate assessment of the osteogenic potential of silanized surfaces was obtained by 
comparing the expression of Col1, ALP and OCN osteogenic gene biomarkers (Figure 4-23 c). It was noted that the gene expression on silanized substrates without collagen collectively differed with the expression trends on the collagen coated substrates. The silanized substrates (without Col1) resulted in higher expression of all three genes. On the contrary, except for APTMS+Col1 substrates which showed a significant upregulation of ALP and Col1 biomarkers, APTES+Col1 and MPTMS+Col1 substrates showed a decrease in gene expression, despite a higher biomineralization. These trends indicate that the col1 immobilization is critical for a cell adhesion and stable cell layer formation but the cell interactions with the underlying silane majorly affect the osteogenic fate of the adhered hMSCs.

From above results, it could be noted that hMSC behaviors are affected by both silane structure and functionality. Silanization could significantly induce changes in surface wettability, roughness and protein retention which are known to significantly affect hMSC adhesion and viability. While the cell behaviors could be primarily influenced by silane type, the immobilization with Col1 could alter surface topography and reactivity which could affect cell adhesion and in stabilizing subsequent behaviors such as proliferation and cell sheet formation. While APTES (with Col1) primary affect initial cell anchorage, APTMS+Col1 result in stabilization of cell adhesion for longer duration ( 21 days) which could majorly affect hMSC proliferation and differentiation. The MPTMS+Col1 resulted in a higher cell adhesion, cell-cell contact which resulting in a highest cell proliferation. However, silanization with MPTMS without Col1 had significant influence on strengthening the initial cell attachment and in driving osteogenic differentiation. This could be attributed to the highly reactive thiol (-SH) groups which can interact with membrane bound proteins as well as the dense layer of positive charges on the trimethyl groups in aqueous solutions. 


\subsubsection{Conclusion}

We report the silanization in regulating hMSC cell fates. Surface reactivity, topography and ECM protein (col1) retention affected by silanization of PDMS are characterized. Eventually the influence of modified surface characteristics on hMSC adhesion, proliferation and potency is investigated. The surface silanization enabled stabilization of cell adhesion and long-term assessment hMSC behaviors. The strategy could also be conveniently implemented for hMSC adhesion and cell sheet study within PDMS microchannel. Moreover, distinct differences in hMSC osteogenic differentiation potential in response to the characteristics of underlying silanes are shown in both flat substrates and miniatured PDMS model. The platform also facilitates a simple, easily fabricable and adaptable method to study cell behavioral regulation to diverse chemical cues. 


\section{References}

[1] Smith, Q. and S. Gerecht, Stem Cell Fate Is a Touchy Subject. Cell Stem Cell. 19(3): p. 289-290.

[2] Gattazzo, F., A. Urciuolo, and P. Bonaldo, Extracellular matrix: a dynamic microenvironment for stem cell niche. Biochem Biophys Acta, 2014. 1840(8): p. 2506-19.

[3] Reyes, C.D., T.A. Petrie, and A.J. Garcia, Mixed extracellular matrix ligands synergistically modulate integrin adhesion and signaling. J Cell Physiol, 2008. 217(2): p. 450-8.

[4] Chastain, S.R., et al., Adhesion of mesenchymal stem cells to polymer scaffolds occurs via distinct ECM ligands and controls their osteogenic differentiation. J Biomed Mater Res A, 2006. 78(1): p. 73-85.

[5] Barker, T.H., The role of ECM proteins and protein fragments in guiding cell behavior in regenerative medicine. Biomaterials, 2011. 32(18): p. 4211-4.

[6] Kim, S.H., J. Turnbull, and S. Guimond, Extracellular matrix and cell signalling: the dynamic cooperation of integrin, proteoglycan and growth factor receptor. J Endocrinol, 2011. 209(2): p. 139-51.

[7] Frantz, C., K.M. Stewart, and V.M. Weaver, The extracellular matrix at a glance. Journal of Cell Science, 2010. 123(24): p. 4195-4200.

[8] Hinderer, S., S.L. Layland, and K. Schenke-Layland, ECM and ECM-like materials Biomaterials for applications in regenerative medicine and cancer therapy. Adv Drug Deliv Rev, 2016. 97: p. 260-9.

[9] Kyburz, K.A. and K.S. Anseth, Synthetic mimics of the extracellular matrix: how simple is complex enough? Ann Biomed Eng, 2015. 43(3): p. 489-500.

[10] Thery, M., Micropatterning as a tool to decipher cell morphogenesis and functions. Journal of Cell Science, 2010. 123(24): p. 4201-4213.

[11] Tang, X., M.Y. Ali, and M.T. Saif, A Novel Technique for Micro-patterning Proteins and Cells on Polyacrylamide Gels. Soft Matter, 2012. 8(27): p. 7197-7206.

[12] Ostuni, E., et al., Using Self-Assembled Monolayers to Pattern ECM Proteins and Cells on Substrates, in Extracellular Matrix Protocols: Second Edition, S. Even-Ram and V. Artym, Editors. 2009, Humana Press: Totowa, NJ. p. 183-194.

[13] Webb, K., V. Hlady, and P.A. Tresco, Relative importance of surface wettability and charged functional groups on NIH 3T3 fibroblast attachment, spreading, and cytoskeletal organization. J Biomed Mater Res, 1998. 41(3): p. 422-30.

[14] Thevenot, P., W. Hu, and L. Tang, SURFACE CHEMISTRY INFLUENCE IMPLANT BIOCOMPATIBILITY. Current topics in medicinal chemistry, 2008. 8(4): p. 270-280.

[15] Arima, Y. and H. Iwata, Effect of wettability and surface functional groups on protein adsorption and cell adhesion using well-defined mixed self-assembled monolayers. Biomaterials,2007. 28(20): p. 3074-3082.

[16] Thames, S.F. and K.G. Panjnani, Organosilane polymer chemistry: A review. Journal of Inorganic and Organometallic Polymers, 1996. 6(2): p. 59-94.

[17] Soteropulos, C.E. and H.K. Hunt, Attaching Biological Probes to Silica Optical Biosensors Using Silane Coupling Agents. Jove-Journal of Visualized Experiments, 2012(63).

[18] Marques, M.E., A.A.P. Mansur, and H.S. Mansur, Chemical functionalization of surfaces for building three-dimensional engineered biosensors. Applied Surface Science, 2013. 275: p. 347-360.

[19] Chu, Z.Y., et al., Highly enhanced performance of glucose biosensor via in situ growth of oriented Au micro-cypress. Journal of Materials Chemistry, 2012. 22(41): p. 2191721922. 
[20] Wu, Y.L., et al., Highly sensitive polysilicon wire sensor for DNA detection using silica nanoparticles/gamma-APTES nanocomposite for surface modification. Sensors and Actuators B-Chemical, 2011. 155(2): p. 709-715.

[21] Truskey, G.A. and T.L. Proulx, Relationship between 3t3 Cell Spreading and the Strength of Adhesion on Glass and Silane Surfaces. Biomaterials, 1993. 14(4): p. 243-254.

[22] Sweetman, M.J., et al., Dual silane surface functionalization for the selective attachment of human neuronal cells to porous silicon. Langmuir, 2011. 27(15): p. 9497-503.

[23] Chen, W.C., Y. Lo, and H.S. Chen, Effects of Ti surface treatments with silane and arginylglycylaspartic acid peptide on bone cell progenitors. Odontology, 2015. 103(3): p. 322-32.

[24] Friend, J. and L. Yeo, Fabrication of microfluidic devices using polydimethylsiloxane. Biomicrofluidics, 2010. 4(2).

[25] Xue, C.Y., et al., Simplest Method for Creating Micropatterned Nanostructures on PDMS with UV Light. Langmuir, 2011. 27(22): p. 13410-13414.

[26] Hautefeuille, M., et al., New perspectives for direct PDMS microfabrication using a CDDVD laser. Lab on a Chip, 2013. 13(24): p. 4848-4854.

[27] Kuddannaya, S., et al., Surface Chemical Modification of Poly (dimethylsiloxane) for the Enhanced Adhesion and Proliferation of Mesenchymal Stem Cells. ACS Applied Materials \& Interfaces, 2013. 5(19): p. 9777-9784.

[28. Chuah, Y.J., et al., The effects of poly (dimethylsiloxane) surface silanization on the mesenchymal stem cell fate. Biomaterials Science, 2015. 3(2): p. 383-390.

[29] Lee, M.H., et al., Effect of biomaterial surface properties on fibronectin-alpha5beta1 integrin interaction and cellular attachment. Biomaterials, 2006. 27(9): p. 1907-16.

[30] Keselowsky, B.G., D.M. Collard, and A.J. Garcia, Surface chemistry modulates fibronectin conformation and directs integrin binding and specificity to control cell adhesion. Journal of Biomedical Materials Research Part A, 2003. 66a (2): p. 247-259.

[31] Arima, Y. and H. Iwata, Effect of wettability and surface functional groups on protein adsorption and cell adhesion using well-defined mixed self-assembled monolayers. Biomaterials, 2007. 28(20): p. 3074-82.

[32] Tidwell, C.D., et al., Endothelial cell growth and protein adsorption on terminally functionalized, self-assembled monolayers of alkanethiolates on gold. Langmuir, 1997. 13(13): p. 3404-3413.

[33] Tegoulia, V.A. and S.L. Cooper, Leukocyte adhesion on model surfaces under flow: Effects of surface chemistry, protein adsorption, and shear rate. Journal of Biomedical Materials Research, 2000. 50(3): p. 291-301.

[34] Scotchford, C.A., et al., Protein adsorption and human osteoblast-like cell attachment and growth on alkylthiol on gold self-assembled monolayers. J Biomed Mater Res, 2002. 59(1): p. 84-99.

[35 Moller, R., et al., DNA probes on chip surfaces studied by scanning force microscopy using specific binding of colloidal gold. Nucleic Acids Research, 2000. 28(20).

[36] Deshpande, K.S., et al., Biofunctionalization and self-interaction chromatography in PDMS microchannels. Biochemical Engineering Journal, 2012. 67: p. 111-119.

[37] Tasviri, M., et al., Amine functionalized TiO2-carbon nanotube composite: synthesis, characterization and application to glucose biosensing. Applied Nanoscience, 2011. 1(4): p. 189-195.

[38] Trzcinska, R., et al., Synthesis and characterisation of PEG-peptide surfaces for proteolytic enzyme detection. Analytical and Bioanalytical Chemistry, 2013. 405(28): p. 9049-9059.

[39] Fujiwara, K., H. Kasaya, and N. Ogawa, Gold Nanoparticle Monolayer Formation on a Chemically Modified Glass Surface. Analytical Sciences, 2009. 25(2): p. 241-248. 
[40] Dickinson, L.E. and S. Gerecht, Micropatterned surfaces to study hyaluronic acid interactions with cancer cells. J Vis Exp, 2010(46).

[41] Wang, Y.Y. and L. Tang, Chemisorption assembly of Au nanorods on mercaptosilanized glass substrate for label-free nanoplasmon biochip. Analytica Chimica Acta, 2013. 796: p. 122-129.

[42] Englade-Franklin, L.E., C.K. Saner, and J.C. Garno, Spatially selective surface platforms for binding fibrinogen prepared by particle lithography with organosilanes. Interface Focus, 2013. 3(3).

[43] Ibarlucea, B., et al., Biofunctionalization of PDMS-based microfluidic systems. 2011.

[44] Bumgardner, J.D., et al., Contact angle, protein adsorption and osteoblast precursor cell attachment to chitosan coatings bonded to titanium. Journal of Biomaterials SciencePolymer Edition, 2003. 14(12): p. 1401-1409.

[45] Marin-Pareja, N., et al., Different Organization of Type I Collagen Immobilized on Silanized and Nonsilanized Titanium Surfaces Affects Fibroblast Adhesion and Fibronectin Secretion. ACS Applied Materials \& Interfaces, 2015. 7(37): p. 20667-20677.

[46] Magyari, K., et al., Attachment and conformational changes of collagen on bioactive glass surface. Bio-Medical Materials and Engineering, 2016. 27(1): p. 63-74.

[47] Noy, A., Handbook of Molecular Force Spectroscopy. Springer US, 2008.

$* * * * *$

Remarks: The above chapter is related to the manuscripts that were submitted to ACS Applied materials and Interfaces and RSC-Biomaterials Science journals for consideration to publish as a research article 


\section{Chapter. 5 Effect of surface chemistry on neuronal cell behaviors}

\subsection{Enhanced In-vitro Biocompatibility of Chemically Modified PDMS surfaces for Stable Adhesion and Long-term Investigation of Brain Cerebral Cortex Cells}

\subsubsection{Background}

Miniaturized cell culture systems offer precise control of cell numbers in a given area or volume for minimalistic as well as single cell investigations, with minimal reagent consumption and cell loss [1-3]. In recent years, poly-dimethylsiloxane (PDMS) has been one of the predominant materials in micro-fabrications of miniaturized study platforms of several cellular processes due to its ease of fabrication, low cost and optical transparency [4-6]. Furthermore, the flexibility and versatility of tunable mechanical and surface topographical6 features up to sub-micron precision has increasingly facilitated rapid-prototyping of cell systems at the exploratory stages of fundamental research $[4,6,7]$. These advantages have been tapped for in vitro study platforms of cell differentiation [8], tumor progression [9], cell-cell/environment interactions [10-12], physiology [13], functional studies $[4,14]$ and disease models $[15,16]$. In most of these studies, in vivo micro-architectures have been mimicked at cellular, tissue and organ level architectures on chip. Of recent interest is to explore complex mechanisms involved in neural stem cell differentiation [17], disease progression [18], high-throughput drug screening [19] and cell-cell interactions of brain [20] on PDMS microsystems. In this regard, there has been a growing interest in the study of primary neural cell cultures from cerebral cortex digest for detailed studies on specific physiological behaviors such as axonal guidance [21], cell maturation [22], cell-surfacechemical/topographical interactions [23], synaptogenesis [24] under different physiologically 
relevant conditions and micro-environments. Moreover, the vital functional roles played by cerebral cortex in memory, higher thinking, sensory and motor perception, have been studied [24]. Neurodegenerative disorders such as Alzheimer's disease, Parkinson's disease and amyotrophic lateral sclerosis have been linked to the changes in biochemical and cellular micro-environments during aging. In all these studies, the high inaccessibility to the brain makes it desirable to study neuronal function and degeneration on appropriate in vitro model system, which requires to closely mimicking the in vivo state with high physiological relevance [25]. Such in vitro models would enhance drug screening and biomarker discovery [26, 27]. The study of the cerebral cortex cell functions on PDMS microchips with suitable design and micro-environmental considerations could offer higher specificity and control with minimal cell and reagent consumption.

However, one of the discouraging factors concerning the use of PDMS systems is its low biocompatibility due to inherent high surface hydrophobicity [28-30]. Attempts have been made to reduce surface hydrophobicity of PDMS [31-33]. Although this problem could be averted for a short time period either by protein absorption or plasma treatment, these effects are transient due to hydrophobic recovery [32,33] leading to eventual detachment and clumping and death of cells within one week of cell seeding [28,34]. Such problems caused by the short term and weaker surface functionalization [34-37] poses serious limitations on any long term in vitro studies concerning synaptogenesis [38], neural aging and death [39], synaptic plasticity [40], and neural information processing [41] on PDMS surfaces.

Synaptic proteins such as synaptophysin, $\beta$-synuclein as well as the glutamate receptors in the cultured neurons reach their maximum expression level starting from 15 to 20 days after cell plating [39]. Moreover, $\beta$-synucleins and $\beta$-amyloids which are some of the key factors in neurodegenerative disease (E.g., Parkinson's and Alzheimer's diseases) progression are known to localize within mature neurons upon growth for 20 days in vitro (DIV) [39]. Furthermore, in vitro neural network matures with culture age and starts to exhibit full electrical activity only after 2 weeks of cell plating $[42,43]$. This indicates that maintaining a long-term culture of primary 
cortical cells is important for a meaningful investigation of cell growth and maturation, neuronal network formation, signaling etc,

Interactions between the proteins and the substrate surfaces are often dominated by weak [44] forces such as the electrostatic, hydrophobic and van der Waals interactions which could cause gradual protein leaching into the culture medium. As a result, the matrix protein support on PDMS surface eventually loses its biocompatibility to promote cell culture. Previous studies have attempted to reduce such effects by introducing stable and strong covalent binding between the proteins and material surfaces [45-47]. Recently, we had shown that covalent cross-linking of ECM proteins could promote enhanced mesenchymal stem cell adhesion, compared to traditional protein adsorption techniques. The resulting PDMS surfaces with lowered hydrophobicity favored stable and long-term anchorage and differentiation of cells [47, 8].

However, to our knowledge, the effect of such modification chemistry on the stability of ECM protein binding and eventual influence on adhesion and behavior of the brain cerebral cortex cells in vitro are not known. Foreseeing a need for stable and functional cortical culture system, in this work, we employed the aminosilane functionalization using APTES for the immobilization of fibronectin, collagen type IV, poly-1-lysine and laminin, proteins (Figure 5-1). These proteins have been commonly studied as key neural cell extracellular matrix (ECM) proteins influencing the cortex cell-microenvironment interactions and as compatible substrate platforms for in vitro cell studies [48-52]. 


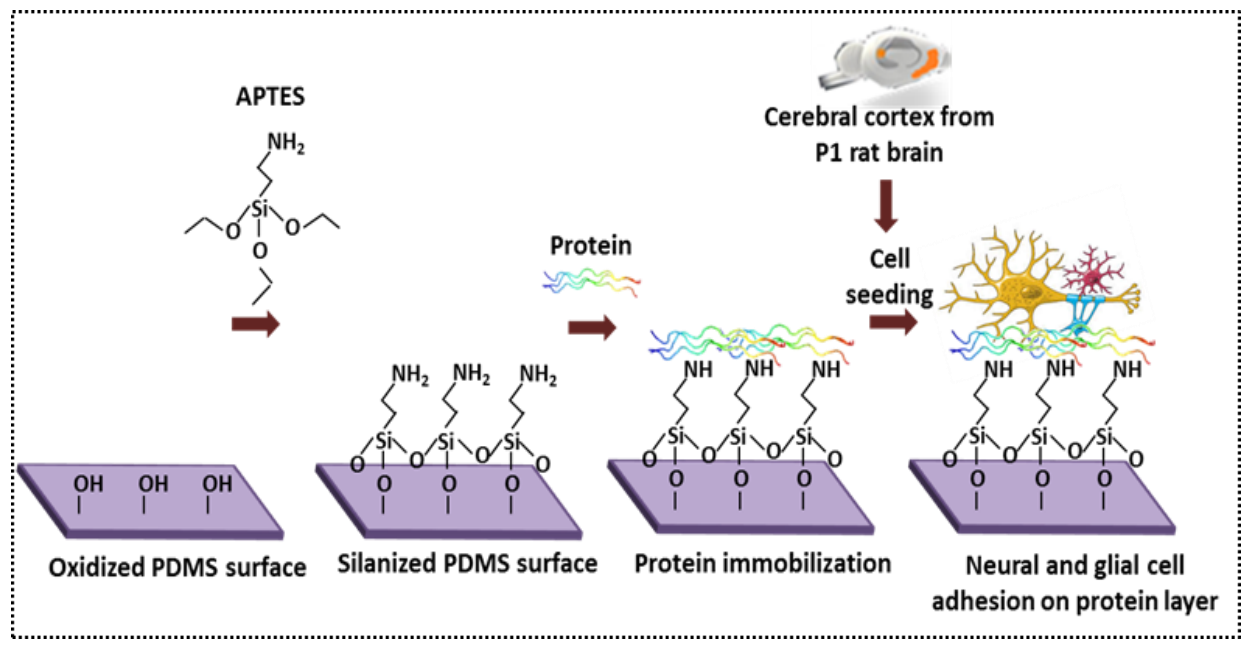

Figure 5-1: Schematic representation of PDMS surface modification for the cerebral cortex cell culture

The proteins interact with charged NH2 domains of APTES by ionic interactions [53]. Subsequently, we perform primary culture of brain cells dissected and digested from the post-natal (P1) rat cerebral cortex on the ECM protein bio-functionalized substrates and assess the adhesion, survival and morphology of neural and glial cell types until 21 DIV. We further test the implementation of this strategy to modify the micro-channels of a widely used PDMS micro-chip with compartmentalized configuration which is a highly adaptable platform to study axonal growth, injury and regeneration, cell-cell interactions, as well as to unravel the chemical and biomolecular signatures of neurons and glial cells $[54,58,63]$. Prolonged analysis of cortical cells in such microsystems could eventually provide deeper insights into the interplay between extracellular signals and the consequent intracellular responses under various conditions concerning the cerebral cortex development and degeneration. 


\subsubsection{Results}

\subsubsection{X-ray Photoelectron Spectroscopy (XPS)}

XPS characterization was performed to reveal the surface elemental composition (Figure 55).

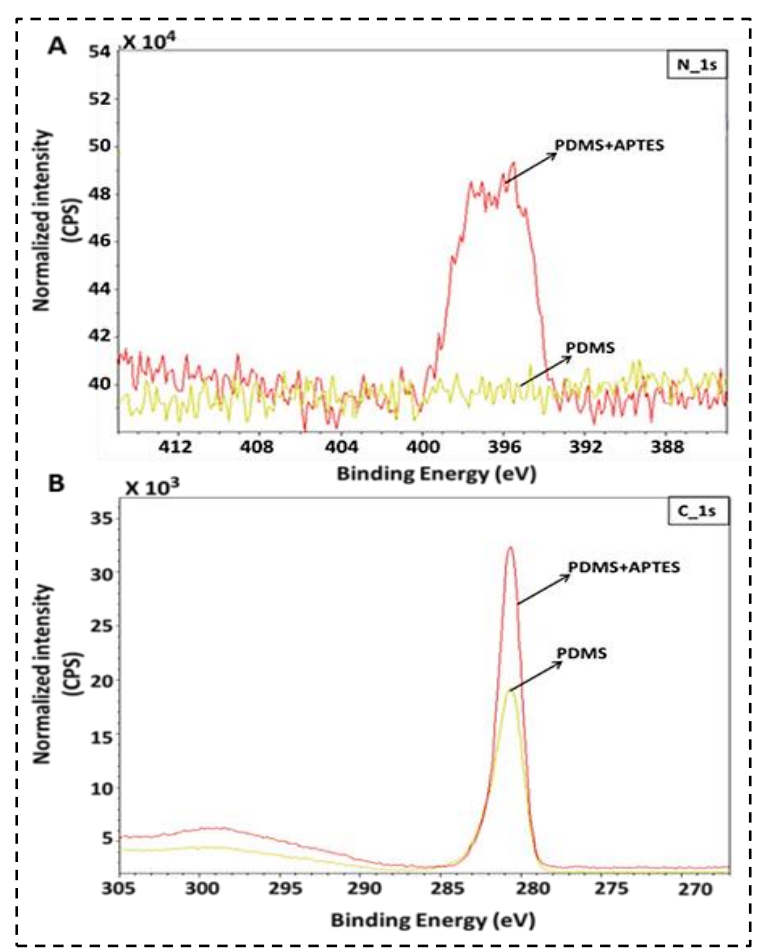

Figure 5-2: Spectral analysis of elemental composition. (A) $N$ 1s (nitrogen) and (B) $C$ 1s (carbon) region XPS scans on native PDMS and APTES treated PDMS substrates.

The relative loading of nitrogen (N1s) (Figure 5-2 A) and carbon (C 1s) (Figure 5-2 B) was found to be higher in APTES modified PDMS surfaces in comparison with the native (unmodified) PDMS, which could be attributed to the presence of amino $\left(-\mathrm{NH}_{2}\right)$ and triethyl $\left(-\mathrm{C}_{2} \mathrm{H}_{5}\right)$ functional groups of APTES respectively on the modified substrates.

\subsubsection{Water contact angle measurements}

The surface wettability of the native PDMS and the chemically modified (with APTES+protein) and unmodified (without APTES modification and protein adsorption) PDMS was evaluated by 
measuring the mean water droplet contact angle of the static drop dispensed on the substrate surface (Figure 5-3).

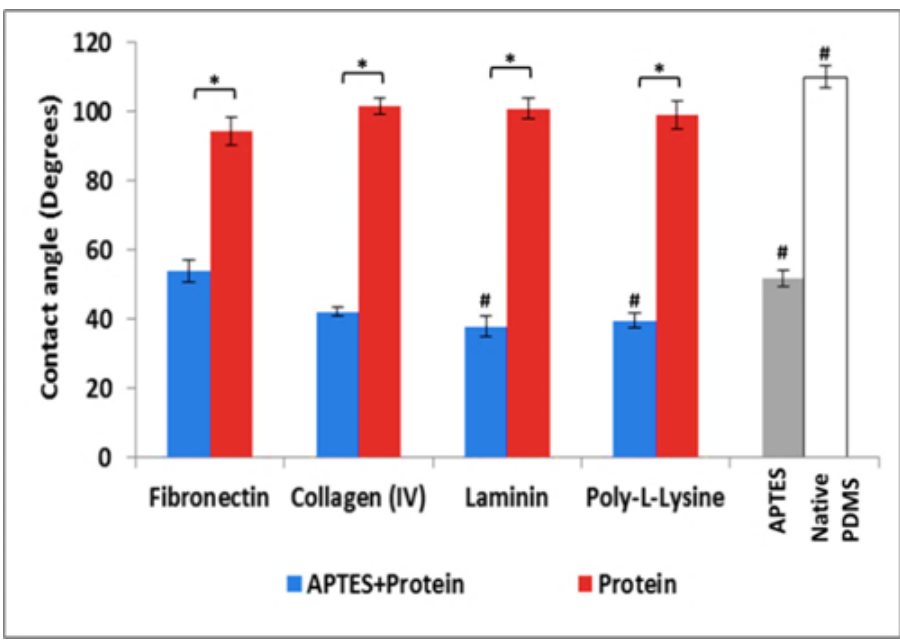

Figure 5-3: Measurements of the average contact angle various PDMS surfaces. The graph is represented as the mean \pm SD. *indicates $p$ values of $<0.05$ between two compared groups. \# indicates $p$ values of $<0.05$ compared to the rest of the similarly treated (APTES+protein) groups.

Lowest wettability was observed on the surfaces of the native PDMS which ranged within a hydrophobic region $\left(110.09 \pm 3.12^{\circ}\right)$ (Figure 5-3). A slight decrease in contact angles were measured on the protein adsorbed PDMS surfaces. However, the surface wettability could still be measured within a hydrophobic range of $\sim 90^{\circ}$ to $100^{\circ}$. However, APTES modified PDMS surfaces with subsequent protein immobilization showed a significant reduction in contact angle at a relatively hydrophilic range for all the tested ECM proteins.

\subsubsection{Protein retention analysis}

To evaluate the degree of protein adsorption on the APTES modified and protein adsorbed PDMS surfaces, a micro BCA analysis was performed (Figure 5-4). 


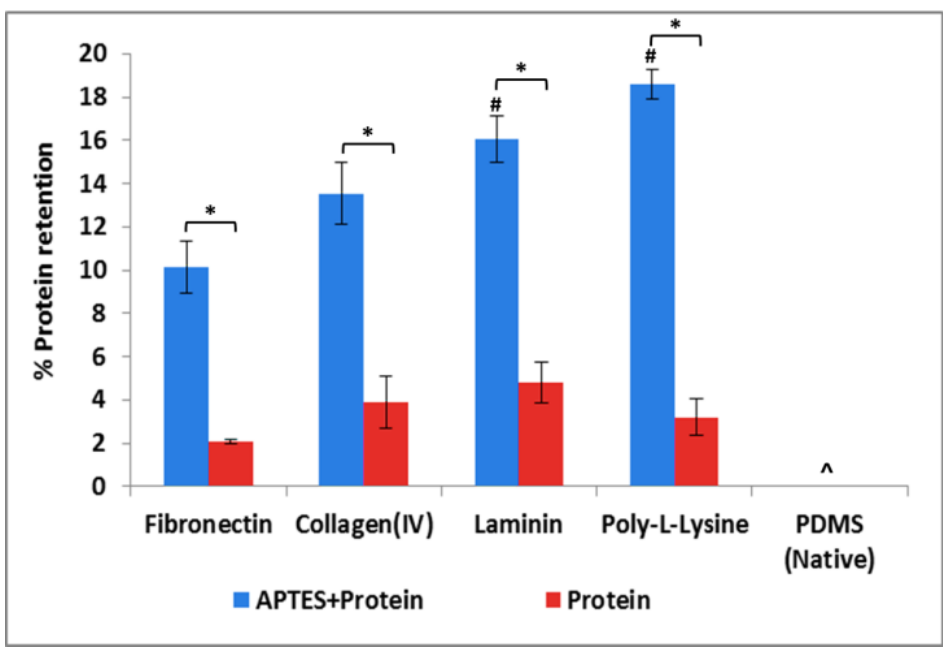

Figure 5-4: Amount of proteins retained on the APTES modified and protein adsorbed PDMS surfaces, calculated from micro BCA assay. The graph is represented as the mean \pm SD. *indicates $p$ values of $<0.05$ between the two compared groups. \# indicates a $p$ value of $<0.05$ compared to the rest of the similarly treated (APTES+protein) groups. ^ indicates zero (nil measurement) on the control substrate.

PDMS surfaces modified with APTES showed enhanced protein retention on the surfaces whereas the unmodified surfaces showed poor protein retention (Figure 5-4). Further, APTES cross-linked laminin and poly-L-lysine surfaces showed relatively stable and higher protein retention compared to similarly modified fibronectin and collagen surfaces. However, protein adsorption on the unmodified surfaces showed a slightly contrasting trend as observed on laminin and poly-l-Lysine adsorbed PDMS surfaces. Among the proteins cross-linked by APTES modification, poly-1-lysine showed highest retention and fibronectin showed a lower retention indicating preferential affinity of proteins towards the functional (- $\left.\mathrm{NH}_{2}\right)$ moieties on modified PDMS surfaces.

\subsubsection{Cortical cell culture}

Cerebral cortex cells were imaged on the APTES modified/unmodified PDMS surfaces during intervals of 3,7, 13 and 21 days in vitro (Figure 5-5). Phase contrast imaging of cells on all the APTES+protein modified plates imaged on 21 st day reveal neuron attachment with a good cell 
viability and neurite formation compared to the unmodified/protein adsorbed PDMS surfaces. In case of the latter, higher cell dislodging, lower cell density and cell agglomeration was observed (Figure 5-5A).

Among the chemically modified surfaces, APTES+laminin and APTES+poly-l-lysine surfaces showed healthier cell adhesion and neural process formation with a well spread cell distribution over the culture area. In contrast, a relatively higher degree of cell dissociation and clumping over time was observed on APTES+fibronectin and APTES+collagen modified surfaces. Live dead assay and Cell viability (Cyquant) assays showed similar trend with a low viability on APTES+collagen surfaces and a relatively lower viability on all unmodified surfaces. APTES+PDMS (without protein) and native PDMS surfaces were found unsuitable for prolonged culture due to early cell dissociation and clumping observed as early as 3 days and 1 day in culture respectively on these surfaces. Similar observations were also reported in previous studies $[4,47$, 66-68].

\subsubsection{Mean neurite length and primary branching}

Neuronal morphology on the modified/unmodified surfaces was characterized by measurement of the neurite length and primary branching around the neural soma. Since neural growth and function are dependent on optimal neuronal attachment, in this study, the neurite density (neurite count per neuron) and mean neurite length were used as indicators of healthy neuron cell adhesion on the substrate surfaces. Hence the neuronal morphology on APTES+protein modified and protein adsorption (without APTES) surfaces was characterized by measurement of the neurite length and primary branching around the neural soma, which shows a relatively higher neurite length (Figure 5-5 B, Row 1) and primary branching (Figure 5-5 B, Row 2) respectively, on the APTES+protein modified surfaces. 


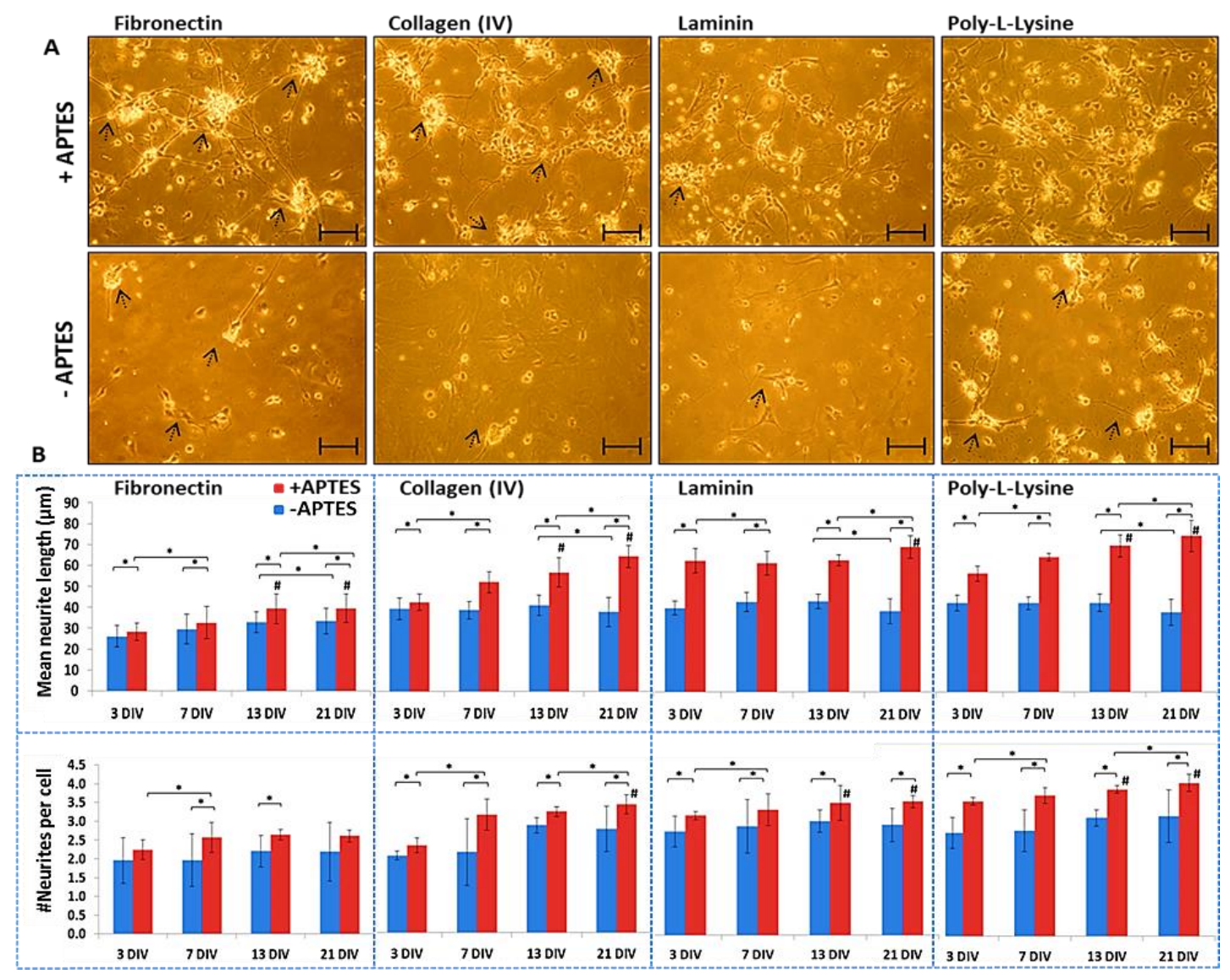

Figure 5-5: (A) Day 21 image set of cerebral cortex cell culture on APTES+protein modified (row 1) and protein adsorbed (row 2) PDMS surfaces. Arrows are pointed to the cell aggregation on surfaces. Scale bar: $100 \mu \mathrm{m}$. (B): Morphological analysis of attached neuronal cells by mean neurite length measurements (row 1) and neurite branch count (row 2) imaged on 3, 7, 13 and 21 DIV on APTES+protein modified (Red) and protein adsorbed (Blue) surfaces. *indicates p values of $<0.05$ between two compared groups. \# indicates $p$ values of $<0.05$ compared to the rest of the similarly treated (APTES+protein) groups.

In general, the unmodified surfaces showed slightly higher standard deviations in the neurite count per cell in all the time points due to greater differences in morphology of neuronal population. Among the APTES modified substrates, APTES+laminin and APTES+poly-1-lysine showed higher neurite length and branching with an increasing trend each measurement time points. For more detailed understanding of the differences in neurite length and neurite count, we studied the distribution of length and branching features on the final time-point of 21 DIV. The data shows 
higher instances of increased neurite growth and branching on APTES cross-linked protein surfaces compared to the unmodified PDMS surfaces. Furthermore, a relatively higher neurite length and branching was observed in both APTES+poly-l-lysine and APTES+laminin surfaces compared to APTES+collagen and APTES+fibronectin surfaces (in descending order).

\subsubsection{Immunostaining and Scanning Electron Microscopy}

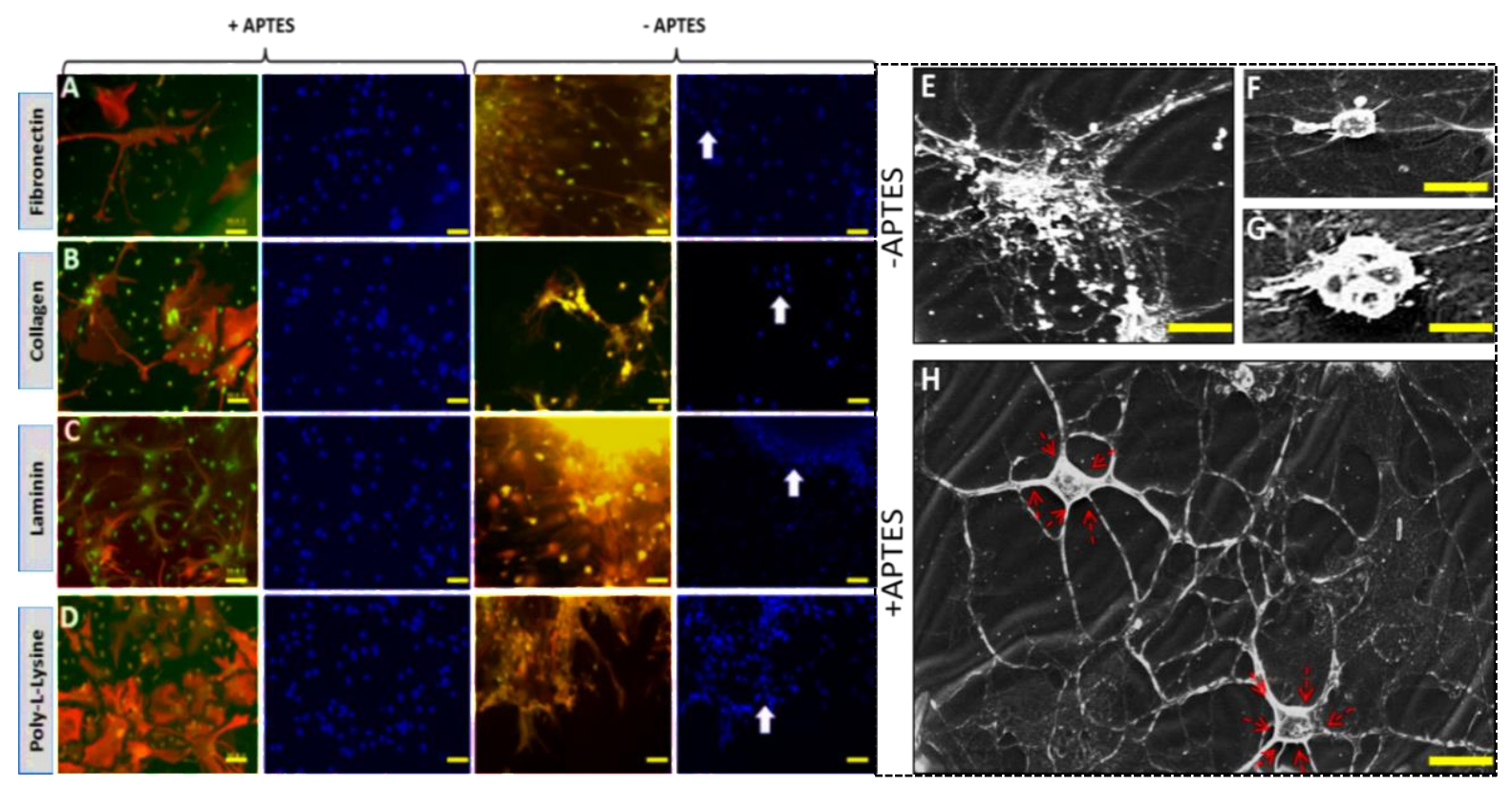

Figure 5-6: Left: Immunostaining of neurons and glial cells (21 DIV) on (A) fibronectin (B) collagen (C) laminin and (D) poly-l-lysine surfaces with (column 1, 2) or without (column 3, 4) APTES crosslinking. MAP2 and GFAP primary antibodies for neurons and glial cells are conjugated with green and red secondary antibodies. DAPI (blue) was used as nuclear stain. White arrows indicate cell clumping sites (Scale bar: $50 \mu \mathrm{m})$. Right: SEM micrographs of (E-G) protein adsorbed and (H) APTES+poly-l-lysine modified PDMS substrates. Red arrows indicate neural branching points (Scale bar: $20 \mu \mathrm{m}$ ).

Although the culture imaging reveals cell growth and neurite details, the complex morphological details of the underlying and surrounding glial cells could be revealed by immunostaining by cell specific biomarker. Particularly, cell type specific immunostaining on MAP2 (conjugated with green fluorescent secondary antibody) and GFAP (conjugated with red fluorescent secondary 
antibody) on primary cortical cells revealed the distribution of neurons and glial cells on the modified/unmodified PDMS substrates respectively (Figure 5-6, A-D).

Generally, the ratio of neural and glial cell population varied with respect to the type of protein immobilized on the PDMS surface. APTES+fibronectin (Figure 5-6 A) surfaces showed a relatively lower glial cell density compared to the similarly immobilized collagen surfaces (Figure 5-6 B). Along with a well spread coverage of neural cells, both APTES+laminin (Figure 5-6 C) and the APTES+poly-1-lysine (Figure 5-6 D) substrates showed higher glial cell coverage with the highest cell coverage on APTES+poly-1-lysine. On the contrary, the cell types on the unmodified PDMS substrates could not be easily differentiated due to a high degree of cell dislodgement and eventual clumping. Hence, an uneven cell distribution with an indiscernible morphology was observed on these surfaces irrespective of the ECM protein type. Detailed investigation of neural cell branching by SEM reveals that on the unmodified poly-1-lysine surfaces, the cells had either unclear or irregular branching (Figure 5-6 E) or clumped up without neurite extensions either as single/grouped cells (Figure 5-6: F and G). On the contrary, the APTES+poly-lysine surfaces showed a healthy neurite branching and morphology (Figure 5-6 H). 


\subsubsection{Long term culture within PDMS microfluidic chip}

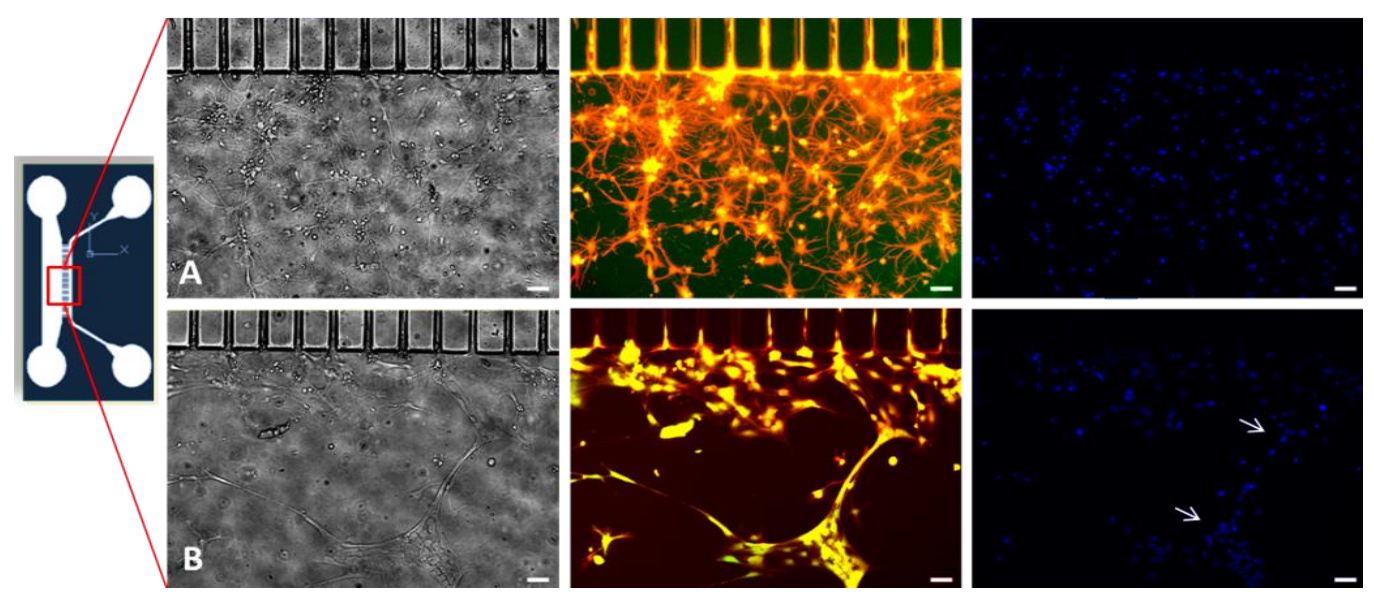

Figure 5-7: Cell culture and immunostaining of neurons (green) and glial cells (red) cultured for 21 DIV on poly-l-lysine immobilized surfaces on two chamber brain cell culture PDMS chip with (A) APTES modification and (B) without APTES modification. The arrows indicated cell clumping sites within the micro-channel surfaces. Scale bar: $50 \mu \mathrm{m}$

Among the proteins tested, poly-l-lysine was chosen for bio-functionalization of PDMS microchannels on a compartmentalized chip due to its improved surface properties and enhanced cell response. Cell loading and medium change cycles on chip were performed with reference to previously tested microfluidic compartmental chip cultures $[54,58]$. The microchip was used to test the implementation of the proposed surface modification on-chip and to subsequently assess the biocompatibility of modified PDMS micro-channels.

Cerebral cortex cells showed improved adhesion within the APTES+poly-1-lysine modified chip compared to the unmodified/protein adsorbed PDMS (Figure 5-7 A and B). The morphology and cell behavior of neuron and glial cells were found to be similar to the cell culture performed on the 2D PDMS substrates. On the contrary, usual cell dislodgement and agglomeration was observed in the unmodified substrates. Although the cells were subject to shear force induced by medium flow during routine medium change cycles, the cell anchorage and growth was shown to be stable on APTES+poly-l-lysine micro-channel surface up to 21 DIV. 


\subsubsection{Discussion}

In recent years, advances in precise micro-environment design and control have greatly enabled in-vitro studies on brain tissue development, cell signaling and function, disease modeling and drug screening in miniaturized platforms. Cell interactions with surface functional moieties and protein type are critical since the surface chemical properties influence cell behavior and morphology. A stronger anchorage of cortical cells on ECM protein bio-functionalized matrix surfaces is important for healthy cell adhesion, neurite formation, cell-cell interaction, maturation, synaptic connectivity, electrophysiology and axo-dendritic polarity. Considering the long-term nature of in vitro investigations involving neural cells, a stable anchorage of cells is desirable. However, high surface hydrophobicity and hydrophobic recovery have posed serious limitations on any long term neurological cell studies on PDMS surfaces. Surface adsorption of matrix proteins or plasma modification of PDMS could only induce transient effects eventually leading to cell dislodgement, uneven spatial distribution of cells, clumping and death. Further, certain anomalies in the native conformation of protein or loose attachment could occur during subsequent washing and routine medium change or perfusion operations which could trigger a depletion of bioactivity of the supportive ECM protein layer [28, 29, 59].

Effective matrix protein immobilization on the substrate is necessary to promote stable cell adhesion at the focal adhesion domains, thus aiding in long-term cell culture $[47,55]$. In this study, we implemented APTES silanization for stable crosslinking of cortical matrix proteins on PDMS. While fibronectin, collagen IV and laminin constitute some of the major proteins of cortical ECM, poly-l-lysine has been widely used for in vitro neural cell cultures due to its charged interaction with proteins and cells [20, 46, 60-62]. Besides, APTES functions as molecular spacers between the protein and the surface, thereby minimizing non-specific/random steric interactions. The rationale for such a cross linking strategy is that silanization in polar solvents expose charged amine $\left(-\mathrm{NH}_{2}\right)$ functionality of APTES to freely interact with abundance of carboxyl terminal $(-\mathrm{COOH})$ at the protein side chains resulting in the formation of stable 
amide/amide like cross-linkages. The XPS spectra (Figure 5-3) on C_1s and N_1s regions confirmed the APTES modification on PDMS because of the presence of triethyl $\left(-\mathrm{C}_{2} \mathrm{H}_{5}\right)$ group revealed by higher carbon loading and amine $\left(-\mathrm{NH}_{2}\right)$ groups revealed by a higher nitrogen loading compared to the unmodified PDMS.

The strength of cell adhesion plays a crucial role in determining the cell viability and growth and maturation. Additionally, certain substrate surface properties such as bio-molecular/bio-chemical composition, wettability, charges, and chemistry are known to influence cell behavior on the substratum surfaces. Previous studies indicate a low degree of neuronal adhesion to the substratum could result in retardation of growth cone formation as well as an overall reduction in the degree of neurite initiation, elongation, and branching [28, 34, 51, 55, 56, 57, 61].

In this regard, one of the dominant factors that could influence cell adhesion on substrate surface is surface wettability. Water contact angles were measured on the APTES modified and unmodified surfaces to correlate the cell (adhesion) against the substratum (hydrophobicity) effects. Particularly, we compared the changes in water contact angles following the protein treatment on APTES modified and unmodified PDMS surfaces for cerebral cortex ECM proteins. A substantial decrease in contact angle was seen in APTES + protein modified surfaces compared to the unmodified/protein adsorbed surfaces indicating the combinatorial role of APTES and protein in mediating (decreasing) surface hydrophobicity (Figure 5-4). Both APTES+laminin and APTES+poly-l-lysine surfaces showed lower contact angles compared to APTES+collagen and APTES+fibronectin surfaces.

Although, APTES+laminin surfaces showed slightly higher wettability compared to APTES+poly-1-lysine, the mean neurite length and neurite branching on these surfaces showed an opposite trend (Figure 5-5 B). This suggested that in addition to surface wettability, cell anchorage on a particular surface could also be influenced by the type of the ECM protein in the immediate microenvironment of the cells. Since these proteins are involved in more direct and specific interactions with the cells, they can mediate the overall cell behavior. Cell adhesion motifs on 
these proteins interact with multiple integrin receptors on cell membrane with varying ligand specificities which could preferentially trigger specific cell behavioral responses [49, 57]. The adhesive interactions between neurons, glial cells and the surrounding extracellular matrix (ECM) mediate several neuronal cell behaviors within the developing cortex. Further, the ECM proteins can induce spatio-temporal changes in expression and function of integrin receptors resulting in changes in the overall cell adhesion behavior. Membrane integrins are also known to mediate both cell-cell and cell-ECM interactions during the development of the cerebral cortex [57]. Previous studies also show the effect of specific functional groups on neural cell growth from progenitor cells in which the presence of amine (-NH2) groups beneficially influenced focal adhesion and cell morphology, supporting the largest cell contact area among other functional groups $[48,55]$.

To test protein binding on PDMS surfaces, the weakly and non-specifically bound proteins were washed off with Tween 20 reagent to assess the relative protein stability of the APTES+protein and protein adsorbed surfaces after 21 DIV under cell incubation conditions. We also tested the protein retention before and after Tween 20 wash for the initial time-point ( 3 days) in culture conditions to assess the amount of proteins dislodged from APTES modified and unmodified (protein adsorbed) substrates. A significant loss of loosely bound proteins after Tween 20 wash was observed on the protein adsorbed surfaces (average $=21.07 \pm 3.63 \%$ ) compared to APTES+Protein surfaces (average=7.80 $\pm 2.24 \%$ ). Higher protein retention on APTES+protein surfaces indicates a higher degree of stable linkages between the protein and substrate in comparison with the protein adsorbed PDMS surfaces. Laminin and poly-l-lysine showed relatively higher stability on APTES modified PDMS. Additionally, collagen bonding showed higher stability than fibronectin on modified surfaces. Relative differences in protein affinity to the substratum surfaces could be attributed to inherent differences in the amino acid distribution in the side chains. Additionally, the overall structural conformation of the proteins could play a part in specific protein-substratum intermolecular interactions. Protein conformation is known to control the organization and dynamics of cell-matrix adhesion $[8,48,56,59]$. Functionalization of 
different matrix proteins with APTES cross-linking on the PDMS surface could offer protein specific preferential orientation and facilitate binding through the affinity moieties on proteins which could specifically bind to the reactive amine(-NH2) groups functionalized on the surface. Furthermore, APTES modification reduced the PDMS surface roughness to a relatively smoother surface topography which could have positively influenced protein binding and subsequent cell attachment. Although APTES treated PDMS surfaces could facilitate initial adhesion of cells due to reduced surface hydrophobicity and probably due to initial interaction between the cell surface charges and APTES self-assembled monolayers, the cell adhesion was generally lower compared to the APTES+protein substrates with also substantial cell loss and clumping within day 3 of culture. Further, the exact mechanisms of cell interactions with self-assembled organosilane monolayers are not clearly established. Native PDMS surfaces were highly hydrophobic and cell dislodgement and clumping occurred within day 1 of culture.

Certain intrinsic ECM protein functional behaviors could also affect cortical cell adhesion [60, 61]. The RGD motifs in fibronectin is known to have high interaction with membrane bound integrins and hence the protein is shown to favor better cell anchorage and viability compared to collagen surfaces. On the contrary, fibronectin had a relatively lower influence on neurite branching. Further, immunostaining of MAP2 antibody of neurons and GFAP of glial cells reveal the functional effects of individual proteins in supporting anchorage and function of specific cell types. APTES modified fibronectin and collagen substrates showed slightly lower attachment of glial cells compared to APTES modified laminin and poly-1-lysine. In general, APTES modified laminin and poly-l-lysine showed notable enhancement of cell adhesion with reduced detachment and clumping coupled with higher neurite branching and lengths. Moreover, APTES+poly-l-lysine surfaces also showed a relatively higher attachment of glial cells along with the cortical neurons, which mimics the in vivo mammalian (rodent) brain cell type distribution [63].

All of the above discussed phenomena point to a wide array of contributing factors, such as higher surface wettability, influence of reactive amine $\left(-\mathrm{NH}_{2}\right)$ groups of APTES on protein conformation 
and immobilization, ECM protein type and their preferential affinity with the substrate surfaces and cell membranal domains which could play a synergistic role in enhancement of cortical cell adhesion. This synergistic effect implies that it is not straightforward to predict which protein modified surfaces are suitable for certain cell culture based on any individual factor. For example, although many cells prefer culture surfaces with high surface energies (i.e. hydrophilic surfaces), neuronal cells require the additional presence of primary anime groups on the growth surface [6466]. Of all the surface proteins, APTES+poly-1-lysine surfaces showed a relatively higher degree of cell adhesion of both neural and glial cell types and higher neurite density. In addition to high wettability and stability seen in our results, poly-l-lysine is known to offer high density of both net positive charge and primary amines which strongly facilitate cell adhesion [20, 46]. Furthermore, these surfaces showed higher number of cells with longer neurites and branching density indicating a healthy cell anchorage and development of neuronal processes. We further tested the implementation of the APTES+poly-1-lysine modification chemistry on a model compartmented PDMS micro-chip, which has been explored for wide array of investigation in neuroscience involving co-culture, axonal injury, disease and drug screening models [54, 58, 63]. On a longterm observation (21 DIV), the modified chip surface showed higher degree of adhesion with optimal adhesion of both cell types while the unmodified channels showed cell dislodgement and clumping, with low cell count which could be attributed to weaker protein attachment and cell loss during routine medium change operations.

\subsubsection{Conclusion}

The high inherent hydrophobicity of PDMS surface restricts cell attachment due to lower wetting and reactivity. Surface chemical modification by APTES cross-linking of ECM proteins on PDMS assists in an enhanced attachment and growth of P1 rat cortical primary cells. Surface wettability, ECM protein retention and protein types influence cell adhesion and morphology in a synergistic interaction. A higher cell density with increased neurite outgrowth and branching was 
observed on APTES+poly-l-lysine surfaces. The surface modification tested on both planar PDMS substrates and PDMS micro-channel surfaces showed stable protein attachment, improved cell adhesion, healthy morphology and growth of both cell types for up to 3 weeks in vitro. The surface modification strategy could prove as a platform for several PDMS based long term neurological system investigations on different microfluidic experimental configurations to tackle various neuroscience questions. 


\section{References}

[1] Halldorsson, S.; Lucumi, E.; Gomez-Sjoberg, R.; Fleming, R. M., Advantages and Challenges of Microfluidic Cell Culture in Polydimethylsiloxane Devices. Biosens. Bioelectron. 2015, 63, 218-231

[2] Velve-Casquillas, G.; Le Berre, M.; Piel, M.; Tran, P. T., Mi-crofluidic Tools for Cell Biological Research. Nano today 2010, 5 (1), 28-47

[3] Zhang, Q.; Austin, R. H., Applications of Microfluidics in Stem Cell Biology. BioNanoScience 2012, 2 (4), 277-286.

[4] Zhang, W.; Choi, D. S.; Nguyen, Y. H.; Chang, J.; Qin, L., Studying Cancer Stem Cell Dynamics on PDMS Surfaces for Microfluidics Device Design. Sci. Rep 2013, 3, 2332:12332:8.

[5] Bartalena, G.; Loosli, Y.; Zambelli, T.; Snedeker, J. G., Bio-material Surface Modifications can Dominate Cell-Substrate Mechanics: the Impact of PDMS Plasma Treatment on a Quantitative Assay of Cell Stiffness. Soft Matter 2012, 8 (3), 673-681

[6] Choi, J. H.; Lee, H.; Jin, H. K.; Bae, J. S.; Kim, G. M., Micro-patterning of Neural Stem Cells and Purkinje Neurons Using a Polydimethylsiloxane (PDMS) Stencil. Lab Chip 2012, 12 (23), 5045-5050.

[7] Hamon, M.; Hanada, S.; Fujii, T.; Sakai, Y., Direct Oxygen Supply with Polydimethylsiloxane (PDMS) Membranes In-duces a Spontaneous Organization of Thick Heterogeneous Liver Tissues from Rat Fetal Liver Cells In vitro. Cell transplant. 2012, 21 (2-3), 401-410

[8] Chuah, Y. J.; Kuddannaya, S.; Lee, M. H. A.; Zhang, Y. L.; Kang, Y. J., The Effects of Poly(dimethylsiloxane) Surface Si-lanization on the Mesenchymal Stem Cell Fate. BiomaterSci-Uk 2015, 3 (2), 383-390.

[9] Ratnayaka, S. H.; Hillburn, T. E.; Forouzan, O.; Shev-koplyas, S. S.; Khismatullin, D. B., PDMS Well Platform for Culturing Millimeter-size Tumor Spheroids. Biotechnol. Progr. 2013, 29 (5), 1265-1269

[10] Hsieh, C. H.; Huang, C. J.; Huang, Y. Y., Patterned PDMS Based Cell Array System: A Novel Method for Fast Cell Array Fabrication. Biomed. Microdevices 2010, 12 (5), 897905

[11] Wong, I.; Ho, C. M., Surface Molecular Property Modifi-cations for Poly(dimethylsiloxane) (PDMS) Based Microfluidic Devices. Microfluid. Nanofluid. 2009, 7 (3), 291-306

[12] Keselowsky, B. G.; Collard, D. M.; Garcia, A. J., Integrin Binding Specificity Regulates Biomaterial Surface Chemistry Effects on Cell Differentiation. P. Natl. Acad. Sci. USA 2005, 102 (17), 5953-5957.

[13] Henle, C.; Hassler, C.; Kohler, F.; Schuettler, M.; Stieglitz, T., Mechanical Characterization of Neural Electrodes based on PDMS-Parylene C-PDMS Sandwiched System. Ieee Eng. Med. Bio. 2011 640-643

[14] Nakazawa, K.; Izumi, Y.; Mori, R., Morphological and Functional Studies of Rat Hepatocytes on a Hydrophobic or Hydrophilic Polydimethylsiloxane Surface. Acta biomater. 2009, 5 (2), 613-620

[15] Tsai, M.; Kita, A.; Leach, J.; Rounsevell, R.; Huang, J. N.; Moake, J.; Ware, R. E.; Fletcher, D. A.; Lam, W. A., In vitro Modeling of the Microvascular Occlusion and Thrombosis That Occur in Hematologic Diseases Using Microfluidic Tech-nology. J. Clin. Invest. 2012, 122 (1), 408-418

[16] Antia, M.; Herricks, T.; Rathod, P. K., Microfluidic Model-ing of Cell-Cell Interactions in Malaria Pathogenesis. PLoS pathog. 2007, 3 (7), e99:0939-e99:0948. 
[17] Beduer, A.; Vieu, C.; Arnauduc, F.; Sol, J. C.; Loubinoux, I.; Vaysse, L., Engineering of Adult Human Neural Stem Cells Differentiation Through Surface Micropatterning. Biomateri-als 2012, 33 (2), 504-514.

[18] Park, J.; Lee, B. K.; Jeong, G. S.; Hyun, J. K.; Lee, C. J.; Lee, S.-H., Three-Dimensional Brain-on-a-Chip With an Interstitial Level of Flow and its Application as an In vitro Model of Alz-heimer's Disease. Lab Chip 2015, 15 (1), 141-150

[19] Li, W.; Xu, Z.; Huang, J.; Lin, X.; Luo, R.; Chen, C.-H.; Shi, P., NeuroArray: A Universal Interface for Patterning and Inter-rogating Neural Circuitry with Single Cell Resolution. Sci. Rep. 2014, 4, 4784:1-4784:7.

[20] Shi, M.; Majumdar, D.; Gao, Y.; Brewer, B. M.; Goodwin, C. R.; McLean, J. A.; Li, D.; Webb, D. J., Glia Co-culture With Neurons in Microfluidic Platforms Promotes the Formation and Stabilization of Synaptic Contacts. Lab Chip 2013, 13 (15), 3008-3021.

[21] Fan, Y.; Xu, F.; Huang, G.; Lu, T. J.; Xing, W., Single Neuron Capture and Axonal Development in Three-Dimensional Mi-croscale Hydrogels. Lab Chip 2012, 12 (22), 4724-4731

[22] Goyal, G.; Nam, Y., Neuronal Micro-culture Engineering by Microchannel Devices of Cellular Scale Dimensions. Bio-med. Eng. Lett. 2011, 1 (2), 89-98

[23] Bugnicourt, G.; Brocard, J.; Nicolas, A.; Villard, C., Nanoscale Surface Topography Reshapes Neuronal Growth in Culture. Langmuir 2014, 30 (15), 4441-4449

[24] Thomas Yeo, B. T.; Krienen, F. M.; Sepulcre, J.; Sabuncu, M. R.; Lashkari, D.; Hollinshead, M.; Roffman, J. L.; Smoller, J. W.; Zöllei, L.; Polimeni, J. R.; Fischl, B.; Liu, H.; Buckner, R. L., The Organization of the Human Cerebral Cortex Estimated by Intrinsic Functional Connectivity. J. Neurophysiol. 2011, 106 (3), 1125-1165

[25] Gordon, J.; Amini, S.; White, M. K., General overview of neuronal cell culture. Methods. Mol. Biol 2013, 1078, 1-8

[26] Ray, B.; Bailey, J. A.; Sarkar, S.; Lahiri, D. K., Molecular and Immunocytochemical Characterization of Primary Neu-ronal cultures from adult rat brain: differential expression of neuronal and glial protein markers. J. Neurosci. Methods 2009, 184 (2), 294-302

[27] Lacombe, O.; Videau, O.; Chevillon, D.; Guyot, A. C.; Con-treras, C.; Blondel, S.; Nicolas, L.; Ghettas, A.; Benech, H.; Thevenot, E.; Pruvost, A.; Bolze, S.; Krzaczkowski, L.; Prevost, C.; Mabondzo, A., In vitro Primary Human and Animal Cell-based BloodBrain Barrier Models as a Screening Tool in Drug Discovery. Mol. Pharm. 2011, 8 (3), 651-663.

[28] Lee, J. N.; Jiang, X.; Ryan, D.; Whitesides, G. M., Compat-ibility of Mammalian Cells on Surfaces of Poly(dimethylsiloxane). Langmuir 2004, 20 (26), 11684-91.

[29] Fuard, D.; Tzvetkova-Chevolleau, T.; Decossas, S.; Tracqui, P.; Schiavone, P., Optimization of Poly-di-methyl-siloxane (PDMS) Substrates for Studying Cellular Adhesion and Motil-ity. Microelectron. Eng. 2008, 85 (5-6), 1289-1293.

[30] McDonald, J. C.; Duffy, D. C.; Anderson, J. R.; Chiu, D. T.; Wu, H.; Schueller, O. J. A.; Whitesides, G. M., Fabrication of Microfluidic Systems in Poly(dimethylsiloxane). ELECTROPHORESIS 2000, 21 (1), 27-40.

[31] Pinto, S.; Alves, P.; Santos, A. C.; Matos, C. M.; Oliveiros, B.; Gonzalves, S.; Gudina, E.; Rodrigues, L. R.; Teixeira, J. A.; Gil, M. H., Poly(dimethyl siloxane) Surface Modification with Biosurfactants Isolated from Probiotic Strains. J. Biomed. Mater. Res. A 2011, 98 (4), 535-543.

[32] Bodas, D.; Khan-Malek, C., Hydrophilization and Hydro-phobic Recovery of PDMS by Oxygen Plasma and Chemical Treatment - An SEM Investigation. Sensor Actuat. BChem 2007, 123 (1), 368-373.

[33] Hillborg, H.; Gedde, U. W., Hydrophobicity Recovery of Polydimethylsiloxane after Exposure to Corona Discharges. Polymer 1998, 39 (10), 1991-1998. 
[34] Forry, S. P.; Reyes, D. R.; Gaitan, M.; Locascio, L. E., Facili-tating the Culture of Mammalian Nerve Cells with Polyelec-trolyte Multilayers. Langmuir 2006, 22 (13), 5770-5775

[35] Wu, M.-H.; Wang, H.-Y.; Liu, H.-L.; Wang, S.-S.; Liu, Y.-T.; Chen, Y.-M.; Tsai, S.-W.; Lin, C.-L., Development of High-throughput Perfusion-Based Microbioreactor Platform Capa-ble of Providing Tunable Dynamic Tensile Loading to Cells and its Application for the Study of Bovine Articular Chon-drocytes. Biomed. Microdevices 2011, 13 (4), 789798

[36] Fu, J.; Wang, Y.-K.; Yang, M. T.; Desai, R. A.; Yu, X.; Liu, Z.; Chen, C. S., Mechanical Regulation of Cell Function with Ge-ometrically Modulated Elastomeric Substrates. Nat. Meth. 2010, 7 (9), 733-736.

[37] Kilian, K. A.; Bugarija, B.; Lahn, B. T.; Mrksich, M., Geo-metric Cues for Directing the Differentiation of Mesenchymal Stem Cells. P. Natl. Acad. Sci. USA 2010, 107 (11), 4872-4877

[38] Valor, L. M.; Charlesworth, P.; Humphreys, L.; Anderson, C. N. G.; Grant, S. G. N., Network Activity-Independent Coor-dinated Gene Expression Program for Synapse Assembly. P. Natl. Acad. Sci. USA 2007, 104 (11), 4658-4663.

[39] Lesuisse, C.; Martin, L. J., Long-term Culture of Mouse Cortical Neurons as a Model for Neuronal Development, Ag-ing, and Death. J. Neurobiol. 2002, 51 (1), 9-23

[40] Fitzsimonds, R. M.; Song, H. J.; Poo, M. M., Propagation of Activity-Dependent synaptic Depression in Simple Neural Networks. Nature 1997, 388 (6641), 439-448

[41] Eytan, D.; Marom, S., Dynamics and Effective Topology Underlying Synchronization in Networks of Cortical Neurons. J. Neurosci 2006, 26 (33), 8465-8476.

[42] Jimbo, Y.; Kasai, N.; Torimitsu, K.; Tateno, T., MEA-Based Spike Recording in Cultured Neuronal Networks. In Frontiers in Biochip Technology, Xing, W.-L.; Cheng, J., Eds. Springer US: 2006, 88-98.

[43] Wagenaar, D.; Pine, J.; Potter, S., An Extremely Rich Rep-ertoire of Bursting Patterns During the Development of Cor-tical Cultures. BMC Neurosci. 2006, 7 (1), 11:1-11:18.

[44 ] Hlady, V.; Buijs, J., Protein Adsorption on Solid surfaces. Curr. Opin. Biotech. 1996, 7 (1), $72-77$

[45] Halliwell, C. M.; Cass, A. E., A Factorial Analysis of Silani-zation Conditions for the Immobilization of Oligonucleotides on Glass Surfaces. Anal. Chem. 2001, 73 (11), 24762483;

[46] Yuan, H. D.; Mullett, W. M.; Pawliszyn, J., Biological Sam-ple Analysis with Immunoaffinity Solid-phase Microextrac-tion. Analyst 2001, 126 (8), 1456-1461.

[47] Kuddannaya, S.; Chuah, Y. J.; Lee, M. H. A.; Menon, N. V.; Kang, Y.; Zhang, Y., Surface Chemical Modification of Poly(dimethylsiloxane) for the Enhanced Adhesion and Pro-liferation of Mesenchymal Stem Cells. ACS Appl. Mater. Inter-faces 2013, 5 (19), 9777-9784.

[48] Cooke, M. J.; Phillips, S. R.; Shah, D. S.; Athey, D.; Lakey, J. H.; Przyborski, S. A., Enhanced Cell Attachment Using a Novel Cell Culture Surface Presenting Functional Domains from Extracellular Matrix Proteins. Cytotechnology 2008, 56 (2), 71-79.

[49] Ali, S. A.; Pappas, I. S.; Parnavelas, J. G., Collagen Type IV Promotes the Differentiation of Neuronal Progenitors and Inhibits Astroglial Differentiation in Cortical Cell Cultures. Dev. Brain Res. 1998, 110 (1), 31-38.

[50] Jun, S. B.; Hynd, M. R.; Dowell-Mesfin, N.; Smith, K. L.; Turner, J. N.; Shain, W.; Kim, S. J., Low-density Neuronal Net-works Cultured Using Patterned Poly-1-lysine on Microelec-trode Arrays. J. Neurosci. Methods 2007, 160 (2), 317-326.

[51] Clark, P.; Britland, S.; Connolly, P., Growth Cone Guid-ance and Neuron Morphology on Micropatterned Laminin Surfaces. J. Cell Sci. 1993, 105, 203-212. 
[52] Kavinsky, C. J.; Garber, B. B., Fibronectin Associated with the Glial Component of Embryonic Brain Cell Cultures. J. Su-pramol. Struct. 1979, 11 (2), 269-281.

[53] Vashist, S. K.; Dixit, C. K.; MacCraith, B. D.; O'Kennedy, R., Effect of Antibody Immobilization Strategies on the Analytical Performance of a Surface Plasmon Resonance-Based Immu-noassay. Analyst 2011, 136 (21), 4431-4436.

[54] Dinh, N.-D.; Chiang, Y.-Y.; Hardelauf, H.; Baumann, J.; Jackson, E.; Waide, S.; Sisnaiske, J.; Frimat, J.-P.; Thriel, C. v.; Janasek, D.; Peyrin, J.-M.; West, J., Microfluidic Construction of Minimalistic Neuronal Co-cultures. Lab Chip 2013, 13 (7), 1402-1412.

[55] Kim, Y. T.; Wu, C. F., Reversible Blockage of Neurite De-velopment and Growth Cone Formation in Neuronal Cultures of a Temperature-sensitive Mutant of Drosophila. J. Neurosci. 1987, 7 (10), 3245-3255.

[56] Interactions of a Neuronal Cell Line (PC12) with Laminin, Collagen IV, and Fibronectin: Identification of Integrin-Related Glycoproteins Involved in Attachment and Process Outgrowth. J. Cell Biol. 1987, 105 (5), 2347-2358

[57] Schmid, R. S.; Anton, E. S., Role of Integrins in the De-velopment of the Cerebral Cortex. Cereb. Cortex 2003, 13 (3), 219-224

[58] Campenot, R. B.; Lund, K.; Mok, S.-A., Production of Compartmented Cultures of Rat Sympathetic Neurons. Nat. Protocols 2009, 4 (12), 1869-1887.

[59] Hytonen, V. P.; Wehrle-Haller, B., Protein Conformation as a Regulator of Cell-matrix Adhesion. Phys. Chem. Chem. Phys. 2014, 16 (14), 6342-6357

[60] Barros, C. S.; Franco, S. J.; Muller, U., Extracellular Matrix: Functions in the Nervous System. Cold Spring Harb. Perspec. Biol. 2011, 3 (1), a005108:1- a005108:24.

[61] Franco, S. J.; Muller, U., Extracellular Matrix Functions During Neuronal Migration and Lamination in the Mammali-an Central Nervous System. Dev. Neurobiol. 2011, 71 (11), 889-900.

[62] Emtage, L.; Gu, G.; Hartwieg, E.; Chalfie, M., Extracellu-lar Proteins Organize the Mechanosensory Channel Com-plex in C. elegans Touch Receptor Neurons. Neuron 2004, 44 (5), 795-780.

[63] Blake, A. J.; Pearce, T. M.; Rao, N. S.; Johnson, S. M.; Williams, J. C., Multilayer PDMS Microfluidic Chamber for Controlling Brain Slice Microenvironment. Lab Chip 2007, 7 (7), 842-849.

[64] Corey, J. M.; Wheeler, B. C.; Brewer, G. J., Compliance of Hippocampal-Neurons to Patterned Substrate Networks. J. Neurosci. Res. 1991, 30 (2), 300-307.

[65] Schaffner, A. E.; Barker, J. L.; Stenger, D. A.; Hickman, J. J., Investigation of the Factors Necessary for Growth of Hippocampal Neurons in a Defined System. J. Neurosci. Methods 1995, 62 (1-2), 111-119

[66] Hardelauf, H.; Waide, S.; Sisnaiske, J.; Jacob, P.; Hausherr, V.; Schobel, N.; Janasek, D.; van Thriel, C.; West, J., Micropat-terning Neuronal Networks. Analyst 2014, 139 (13), 3256-3264

[67] Kleinfeld, D.; Kahler, K. H.; Hockberger, P. E., Controlled Outgrowth of Dissociated Neurons on Patterned Substrates. J. Neurosci. 1988, 8 (11), 4098-4120.

[68] Millet, L. J.; Stewart, M. E.; Sweedler, J. V.; Nuzzo, R. G.; Gillette, M. U., Microfluidic Devices for Culturing Primary Mammalian Neurons at Low Densities. Lab Chip 2007, 7 (8), 987-994. 
Remarks: The above chapter is related to the manuscript that was submitted to ACS Applied materials and Interfaces journal for consideration to publish as a research article 


\section{Chapter. 6 Effect of inclusion of topographic elements on hMSC behaviors}

\subsection{Combined effects of multi-topographical and surface chemical environment on mesenchymal stem cell fates}

\subsubsection{Background}

Cell-surface interactions constitute the basic principles of synchronous relationship between cells and biomaterials which needs to be thoroughly understood to facilitate efficient tissue repair and regeneration strategies. The self-renewal property of bone marrow Mesenchymal Stem Cells (hMSCs), has been increasingly utilized to tune their lineage into several tissue specific cell types such as osteoblasts [3-7], adipocytes [8-12], myoblasts [13-16] and chondrocytes [17-20] using wide variety of differentiation factors and extra cellular material/interfacial stimuli. Besides, of the reportedly lower level of immunogenicity offered by hMSCs has been an attractive feature for potential transplantation based therapies [21, 22].

In vivo, critical cell behaviors, including cellular stresses, proliferation, migration, and differentiation are dominated by signals from their surrounding micro-environment [23]. In this regard, it is vital to study the cell-material interactions in terms of both the underlying material surface chemistry and the surrounding physical/mechanical cues which dictate key cellular behaviors. Optimal interaction of the trans-membranal integrins of hMSCs with the underlying proteins and biochemical cues in the extra-cellular matrix is a key factor in regulating their vital behaviors such as adhesion, proliferation, long term survival, migration and differentiation [2325]. With a focus on surface chemical regulation of stem cell fates, past studies have highlighted 
the importance of interactive functional groups and extracellular matrix proteins in influencing cell behaviors [26-29]. Recently, we optimized the surface bio-compatibility of PDMS by silanization based cross-linking strategy to improve surface wettability and subsequent ECM protein immobilization which significantly improved hMSC adhesion [2]. This platform enabled direct investigation of hMSC fates in response to the underlying surface chemistry with detailed characterization of various cell-surface chemical/functional group interactions that govern long term cell adhesion, proliferation and osteogenic differentiation. Controlling cell fates through external cues has thus been a core focus of translational regenerative therapies to direct stem cells to perform specific environment directed functions.

Besides the bio-chemical composition of the ECM and surface chemical functionality, the hMSCs have also been shown to be sensitive towards the physical and mechanical factors such as topography [30-33], rigidity [34-36] and roughness [34, 37] of the underlying substrate. Predominantly, the physical properties in the close vicinity of hMSCs such as micro and nanoscale topographical cues are known to trigger specific cell responses [33, 38, 39]. To mimic such micro-environments in vitro by considering the in vivo complexities is desirable to accurately regulate stem cell self-renewal and differentiation towards specific therapeutic end results. Micro and nano scale fabrication technology have been extensively exploited to precisely manipulate the substrate microarchitecture and eventually to guide hMSCs behaviors. For instance, Engel et al., have found that the adhesion of rat hMSCs was significantly favored on micro-ring shape patterns on poly(methyl methacrylate) (PMMA) to compared to other microstructures [40]. Lee et al. showed that the cell spreading and proliferation of human hMSCs were enhanced by island-like micropatterns with $100 \mu \mathrm{m}$ diameter [41]. In addition, myogenic differentiation of human hMSCs could be promoted by micro-grooved surface by RhoA/ROCK pathway activation and cell shape elongation [42] while geometrical variations (e.g. holly leaf) or honey comb like microstructures orchestrated osteogenic differentiation by WnT signaling [31, 43]. 
Considering the formidable in vivo complexity of both the biochemical and physical environments of the stem cells, it is critical to investigate the comprehensive effects of surface chemistry as well as the topographical signals for rational and optimal tissue regeneration. However, most in vitro models do not adequately address the combinatorial influences of substratum properties on hMSCs behaviors. This necessitates the design of suitable platforms wherein the individual and synergistic influences of both surface chemical and micro topographical could be suitably varied and the subsequent influence on hMSCs fates could be effectively studied.

The high tuneability of PDMS based platforms have been predominantly employed in several cell based studies especially involving well-defined micro/nano-patterning and microfluidic fabrications [44-47]. In addition to favorable properties such as optical transparency, gas permeability and low cost, most importantly, PDMS could be molded into sub-micrometer-scale patterns by soft lithography which enables rapid prototyping of micron scale physical complexity of stem cell niches. This feature has been explored in studies which have focused solely on the influence of physical micro/nano mechano-cues in driving stem cell proliferation and differentiation [30, 32, 47, 48]. 


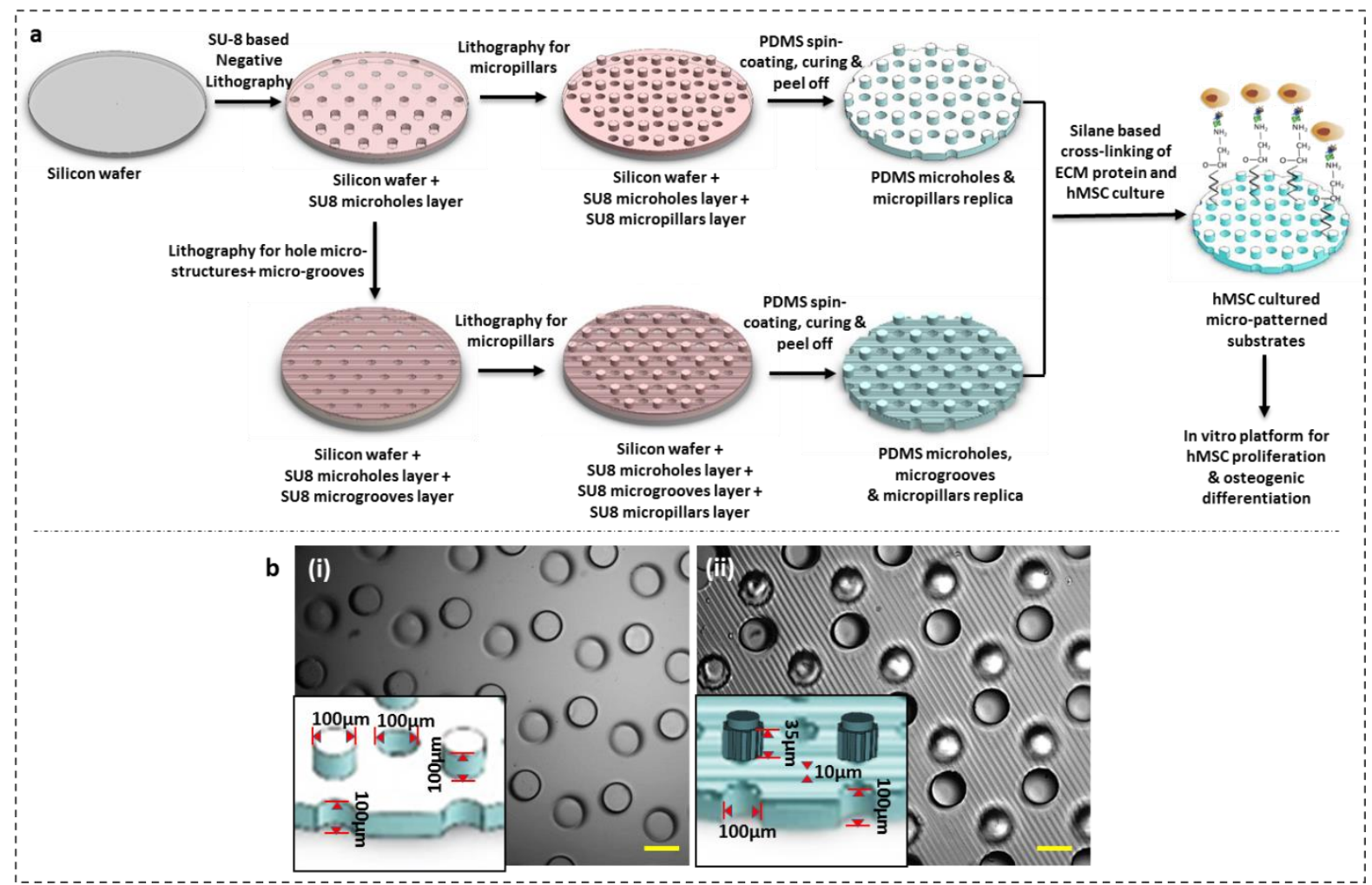

Figure 6-1: (a) Fabrication steps involved in generating PDMS based hole/pillar and groove architectures and subsequent surface modification by covalent linkage of protein (Col1) by silane (APTES) and glutaraldehyde based crosslinking chemistry. (b) Brightfield images of the fabricated (i) hole-pillar (H-P) and (ii) hole-pillar and groove (H-P-G) structures (inset: dimensions of topographical elements). Scale bar: $100 \mu \mathrm{m}$

In order to investigate the comprehensive effects in this work along with the previously dealt surface chemical modification, we studied the inclusion of complex physical/topographic cues on PDMS substrates by employing a novel microfabrication strategy (Figure 6-1). Previous studies have extensively focused on various individual topographical features of various geometry and regularity in the range of $2 \mathrm{~nm}-15 \mu \mathrm{m}$ or with slightly higher physical features such as micropillars/micro brushes $[43,46,48]$. However, since the in vivo bio-mimicking involves a combination of such factors, most studies offer limited, restricted and isolated view of cell behaviors to a certain premeditated physical cue signals. Hence, it is unclear how these physical properties collectively influence formation of hMSC cell sheets right from the cell adhesion, 
proliferation and subsequent tissue development. Moreover, there has been a sparse understanding of hMSC responses to bio-chemical cues in such models due to the overseeing of the underlying surface chemistry which is an essential contributing factor which mediates adhesion and long term cell survival and differentiation.

To address these issues, we aim to investigate the combinatorial role of microstructures and surface chemical modification on adhesion, proliferation and differentiation of hMSCs. Specifically, to include a wide gamut of micro-cue signals, we prepared a hole, pillar and microgroove based micro architecture on PDMS with followed by surface chemical modification for subsequent hMSCs culture on these substrates. Our findings indicate a constructive synergistic role of both microscale physical structure and surface chemistry in forming stable hMSCs cell sheets with practical relevance towards engineering novel, versatile and rational biomaterial/interface for tissue repair and regeneration. 


\subsubsection{Results}

\subsubsection{Cytoskeletal profile and surface coverage of adherent cell layers}

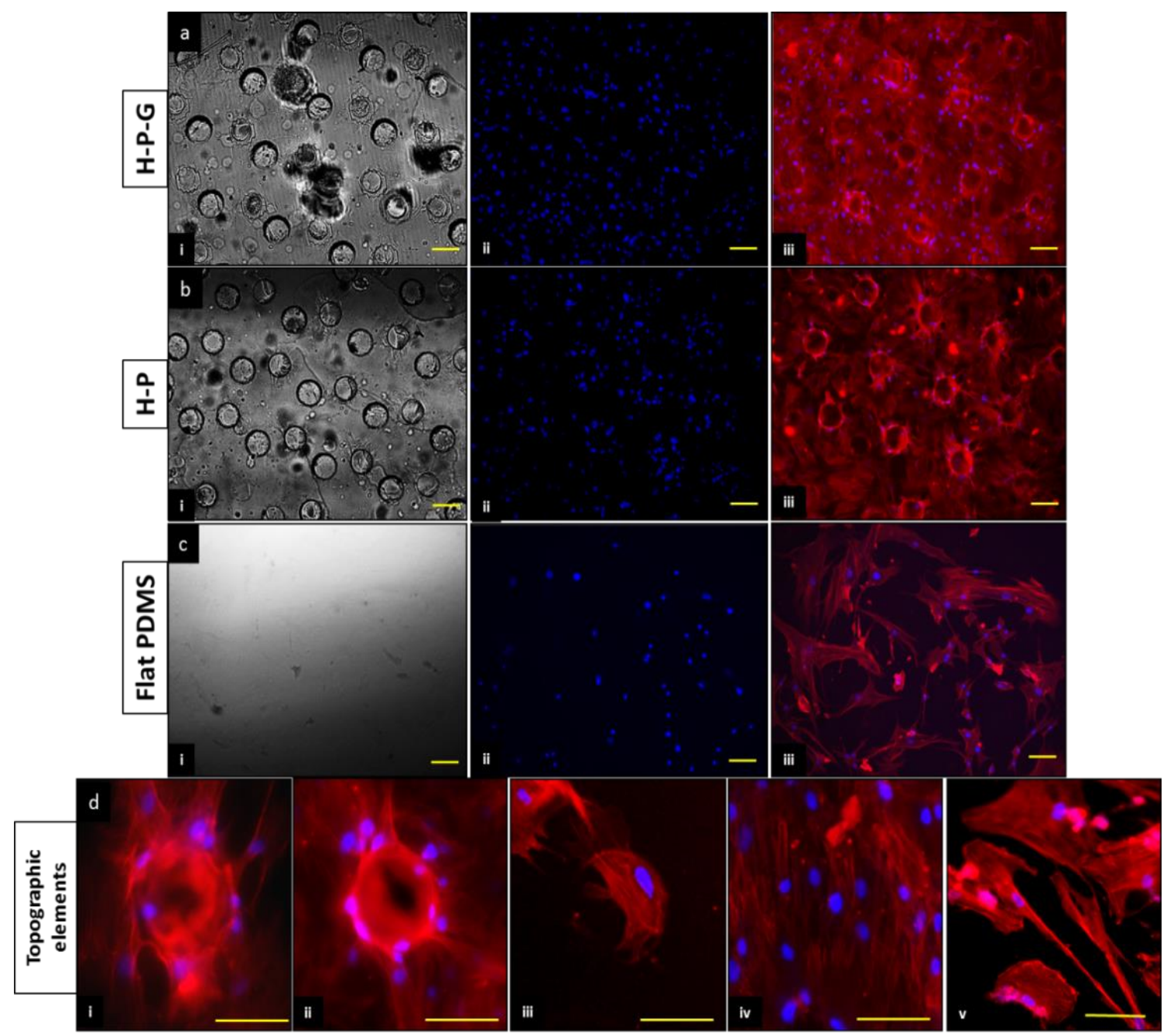

Figure 6-2: Cytoskeletal (F-actin) and nuclear (DAPI) staining of adhered hMSC cell layers (Day 14) on (a) Hole-pillar and groove (b) Hole and pillar (c) Flat PDMS substrates. (i) Brightfield (ii) DAPI and (iii) F-actin+DAPI images. (d) F-actin+DAPI profiles of cells around (i) ridged pillar (ii) smooth pillar (iii) hole (iv) TCP (v) plain PDMS individual topographical elements. Scale bar: $100 \mu \mathrm{m}$

Analysis of cell layer adherence by F-actin cytoskeletal staining revealed the cell cytoskeletal preferences on various substrates. Irrespective of the underlying topography, a healthy adhesion of cells could be observed in all substrates with well spread morphology owing to the reduced hydrophobicity and biocompatibility affected by the APTES+GA based surface chemical 
modification to covalently crosslink Col1 [2]. However characteristic differences in adhesion profiles were observed in response to the dominating topographical elements in the immediate vicinity of the hMSCs. The hMSC cell sheet formed on the H-P-G substrates displayed a consistent surface coverage with almost a uniform cell distribution (Figure 6-2 a(ii)) and closely knit cell-cell contacts (Figure 6-2 a(iii)). This could be attributed to the dominant presence of either the surface grooves or the grooved pillar features which can provide adhesive cues for focal adhesion. Similarly, the H-P substrates (without grooves) also showed a fair cell distribution with comparatively lower surface coverage than on the H-P-G substrates. Stem cell preference to adherent topographic cues are also seen with the hMSCs preferring to attach on nearest pillars, since the pillars are the only dominant topography in this substrate (Figure 6-2 b(iii)). As a result, an increased grouping of the cells around the pillars could be seen (Figure 6-2 b (ii), d(ii)) which resulted in reduced overall cell-cell contact in other regions of the substrate. There were no major differences in the hMSC morphology on the recurring holes present in both H-P-G and H-P substrates. However, obvious preference to pillars instead of holes is evident in H-P substrates. The hMSCs preferences to individual topographic elements showed differences in nucleus distribution and cell attachment in response to the surrounding cues. A noteworthy feature is the preferential attachment of cells at the pillar grooves and the curved surfaces instead of the inner protrusions in H-P-G and H-P substrates respectively (Figure 6-2 d (i) and (ii)). hMSCs sheets partially (sometimes almost fully) cover the holes while anchoring around the edges of the holes often with a stretched morphology (Figure 6-2 d(iii)). On the grooves, the hMSCs showed a higher surface coverage and orientation of actin filaments mostly along the direction of the groove (Figure 6-2 d (iv)). In contrast, although the flat PDMS supported healthy cell adhesion, a relatively lower degree of cell coverage, cell orientations and degree of cell-cell contacts were seen on these substrates (Figure 6-2 c and d (v)). 


\subsubsection{Morphological analysis of hMSCs on topographic substrates}

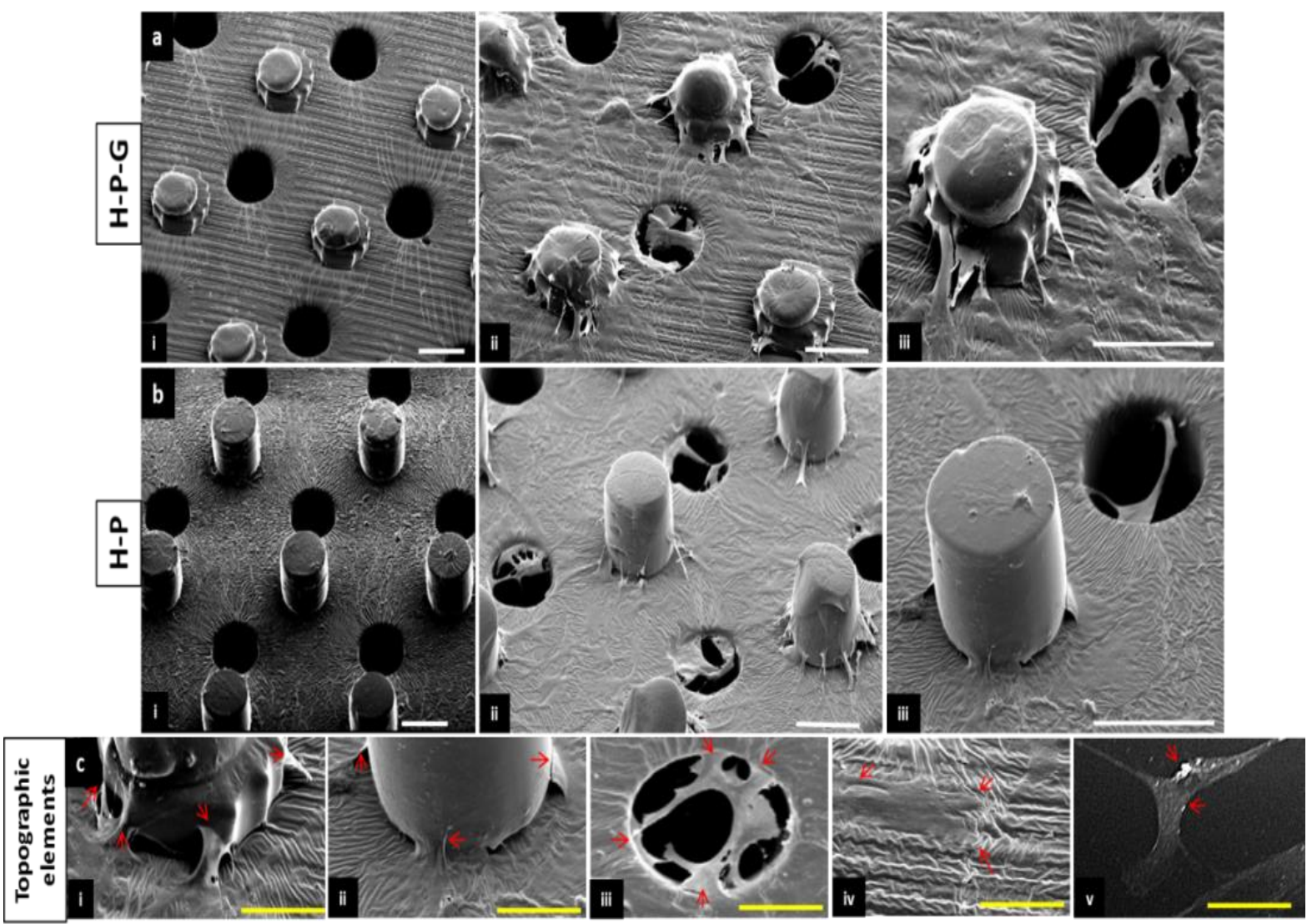

Figure 6-3: (a, b) SEM micrographs of hMSC adhesion and cell sheet formation preferences on H-PG and H-P substrates: (i) un-seeded substrates (ii) hMSC adhered regions and (iii) close-up of cell anchorage on pillar and holes. (c) comparison of cell anchorage points in response to individual topographic elements. Scale bars (White: $100 \mu \mathrm{m}$, Yellow: $50 \mu \mathrm{m}$ )

The SEM micrographs revealed the detailed morphology of the adhered hMSCs on the substrates. The hMSC cell layers on the H-P-G substrates showed diversity in adhesive morphology with respect to the nature of contacting topography. The hMSCs appeared to both climb over and cling to the angled edges rather than on the inner crevices of the ridged pillars (Figure 6-3 a). While anchoring around the pointed ridges, the cells also assumed a well spread and stretched morphology appearing to flow into the grooved support underneath these pillars (Figure 6-3 a (iii), c (i)). On the grooved surfaces, the cells showed a highly-spread profile with strong adherence on grooves compared to relatively random adherence and minimal adhesion junctions of hMSCs 
prevalent on flat PDMS (Figure 6-3 a(iii), c(iv)). The isotropic curved surface of the cylindrical pillars in H-P structures also supported cell anchorage with multiple climbing and clinging points (Figure 6-3 b). Although a strong cell anchorage is evident around these pillars, the advancing cell layers appeared thinner with relatively lower spreading morphology as compared to the H-P-G substrates (Figure 6-3 b, c (i)). Consistent with the F-actin profiles, the hMSC morphology on holes in both H-P-G and H-P substrates showed strong anchorage points at the edges of the holes while the cell body covered a major portion of the hole opening. Hence the cells on these holes could be imagined as accessing the medium components both above and below the cell body during the course of cell growth and differentiation processes.

\subsubsection{Cell adhesion}

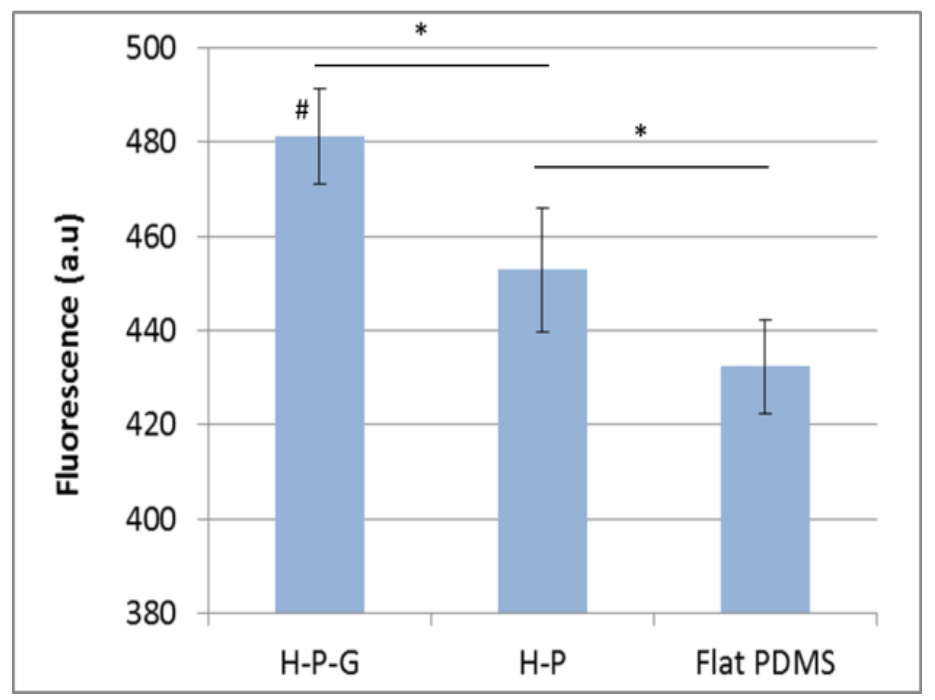

Figure 6-4: Comparison of cell adhesion on H-P-G, H-P and flat PDMS substrates. * indicates p-value $<0.05$ between the compared groups. \# indicates $p$ value $<0.05$ in comparison with all the groups.

To assess the influence of the micropatterns on the initial adhesion of hMSCs, the relative levels of adherent cells were estimated by Cyquant ${ }^{\circledR}$ assay within 90 min of the initial cell seeding (Figure 6-4). Consistent with cell coverage observed from F-actin and cell anchorage profiles, the micropatterned (H-P-G and H-P) substrates showed enhanced adhesion of hMSCs compared to the 
flat PDMS. Previously we had shown an enhanced degree of cell adhesion on chemically modified PDMS surfaces in comparison to the unmodified substrates which showed a rather poor cell adhesion [2]. In this work, the inclusion of topographical features resulted in a positive influence in further enhancing cell anchorage which could be largely attributed to the availability of multiple anchorage points and junctions which are known to affect the integrin signaling and focal adhesion formation. What is further evident is that the H-P-G substrates displayed a significantly higher cell adhesion compared rest of the groups. The presence of intermediate grooves and the ridge structures could not only result in closely packed cell-cell contact but also significantly influence cell adhesion. Since the nature of cell adhesion is known to critically affect the subsequent cell processes such as proliferation and differentiation, it is desirable to know the detailed influences of our micro-topographical features on healthy cell sheet formation.

\subsubsection{Cell proliferation on micro-topographic supports}

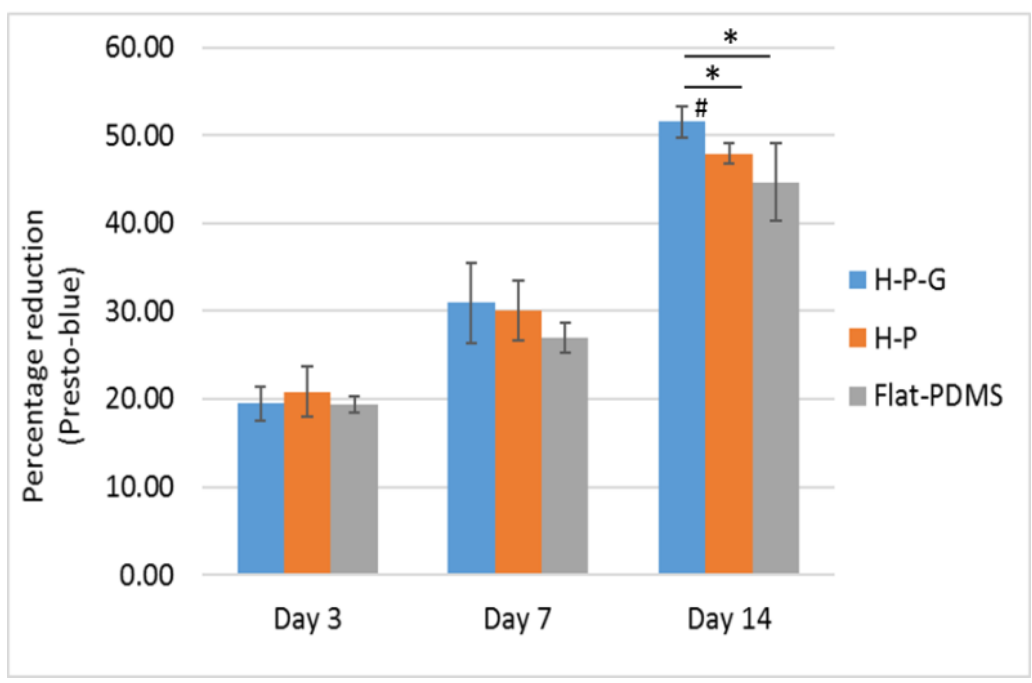

Figure 6-5: Prestoblue analysis of the hMSC proliferation on micropatterend (HPG and HP) and flat PDMS substrates measured at 3,7 and 14 day intervals. * indicates p-value $<0.05$ between the compared groups. \# indicates $\mathrm{p}$ value $<0.05$ in comparison with all the groups.

The proliferation of hMSCs on micropatterned and un-patterned (flat) PDMS surfaces was evaluated with Presto Blue ${ }^{\circledR}$ Cell Viability Reagent on day 3, 7 and 14 respectively. As 
previously observed, the hMSCs cultured on the APTES + GA + collagen modified flat PDMS surface showed persistent cell activity with an increasing proliferation trend unlike the unmodified PDMS substrates [2]. However, both the H-P-G and H-P micropatterned substrates showed an increasing proliferation trend with significantly higher proliferation levels compared to the flat PDMS substrates (Figure 6-5). This significant shift in supporting higher proliferation in micropatterned substrates was observed from Day 7 until Day 14. Among the micropatterned substrates, the H-P-G substrates showed most dominant proliferation trend which significantly differed from other groups on both day 7 and 14. These trends agree with the morphological, adhesive and surface coverage trends elicited by these substrates discussed in the previous sections. This shows that the micropatterned substrates offer favorable support for enhanced focal adhesion which eventually results in an overall enhanced proliferation trend.

\subsubsection{Differentiation to osteogenic lineage}

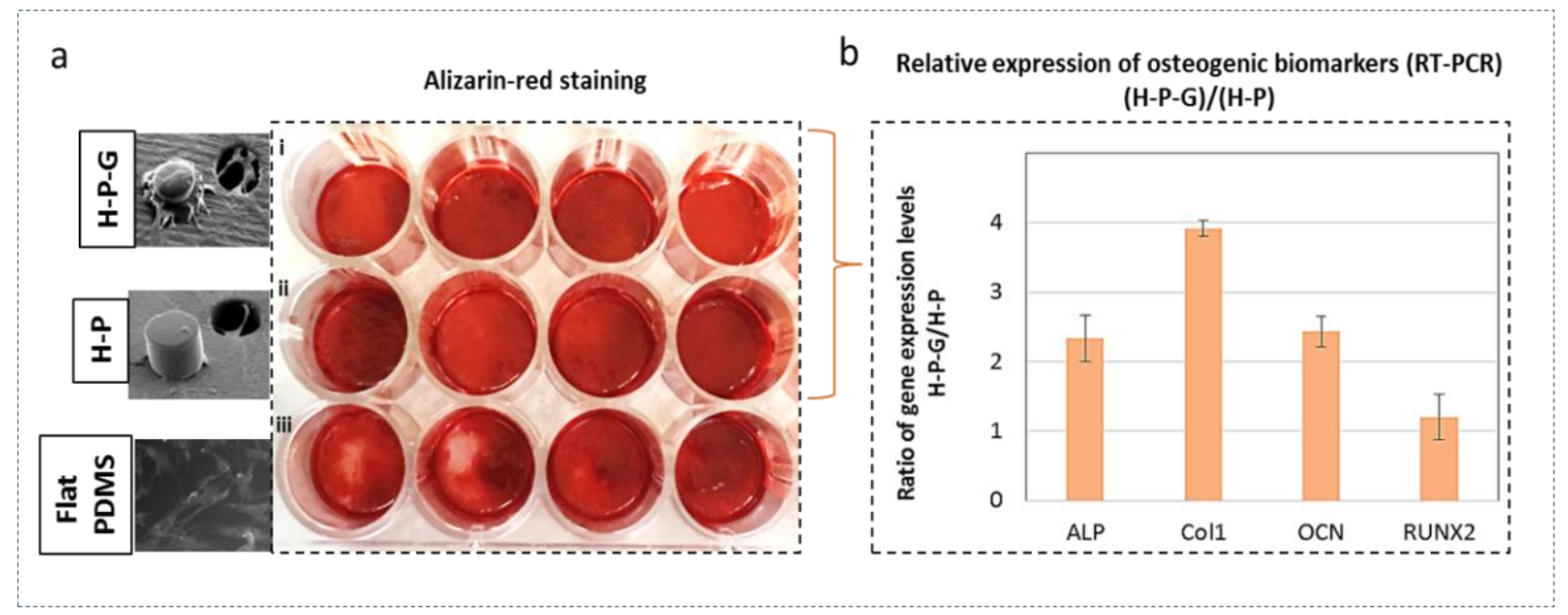

Figure 6-6: (a) Assessment of calcium bio-mineralization following the osteogenic differentiation of hMSCs on micropatterned (HPG and HP) and un-patterned (Flat PDMS) substrates treated with the calcium specific alizarin red dye (D21). (b) Ratio of relative expression of osteogenic genes on H-P-G and H-P substrates. 
Previously, the immobilization of Col1 by APTES+GA based chemical crosslinking was shown to result in a decrease of surface hydrophobicity, thereby stabilizing the cell adhesion with active cell-cell contacts which is an important feature to promote osteogenic differentiation [2]. In this work, the assessment of bio-mineralization by Alizarin red staining after 21-day osteogenic induction revealed that the micro-patterned H-P-G and H-P substrates promoted even higher Ca mineralization during osteogenesis. However, the extent of mineralization in H-P-G and H-P substrates could not be easily distinguished by staining (Figure 6-6 a). Further it could be noted that the cell sheets remained strongly adhered on the micro-patterned substrates (Figure 6-6 a(i) and ii) throughout the 21-day osteogenic culture whereas in one or two of the (chemically modified) flat PDMS substrates, we observed a slight peeling off/dislodgement of cell layer (Figure 6-6 a(iii)). Hence, to obtain a reliable quantitative assessment of osteogenic potential of these substrates, with a clear distinction between the HPG and HP groups, the relative expression of osteogenic biomarkers on these substrates was assessed. In this regard, we chose to study the relative levels of expression four commonly studied biomarkers i.e alkaline phosphatase (ALP), Col1, osteocalcin (OCN) and Runx2. Due to slight non-uniform cell sheet formation on flat substrate, the concentration of mRNA isolated on Day 21 had large variations. Therefore, we compared the relative gene expression between H-P-G and H-P substrates in which a significantly higher fold expression of all the four biomarkers were observed on H-P-G substrates (Figure 6-6 b). The relatively higher fold gene expression on microfabricated PDMS provides a comparative basis of osteogenic potential of microfabricated substrates and emphasizes the higher osteogenic potential of the groove based H-P-G substrates.

\subsubsection{Discussion}

There has been a growing interest to study of vital cell processes such as stem cell differentiation, tissue formation and organogenesis within microfluidic and micropatterned biomimicking interfaces [50-52]. The inherent therapeutic properties of human mesenchymal stem cells 
(hMSCs) are being efficiently tapped by studying the surface chemical and physical factors guiding their survival and multilineage differentiation within carefully controlled artificial environments [53-55]. Due to mechanical tunability and rapid prototyping of micro/nano scale tissue-like architectures, polydimethylsiloxane (PDMS) based platforms are extensively utilized in such studies [56]. With regard to the regulatory effects of the underlying surface chemistry, we had recently shown the influence of ECM protein covalent cross-linking in improving the biocompatibility of PDMS and promoting healthy hMSC adhesion, growth and differentiation [2]. The chemical modification also promoted changes in the nano-scale surface roughness of the material which could significantly affect cell behaviors [1, 2]. Apart from the influence of nanoscale physical characteristics, the hMSC mechanobiology is also critically influenced by micro-scale features which modulate their adhesion and activate subsequent signaling pathways that control their survival, migration and differentiation. Charest et.al, 2006 had shown that cell morphology and adhesion can be strongly influenced by physical topography which can often overrun chemical topography (generated by ECM microprinting) [57]. The size/strength/composition of focal adhesion are sensitive to changes in the topography of the underlying substrate which activate RhoA/ROCK activation of cell cytoskeletal fibers and further regulate subsequent cell processes [58]. Recently, in separate studies Abagnale et.al and Shi et.al, showed the influence of micron scale ridges in the range of 2-15 $\mu \mathrm{m}$ grooves/ridges on hMSC cell spreading and orientation which can regulate adipogenic and osteogenic differentiation [59, 60]. Similarly, holes of various dimensions are shown to affect hMSC behaviors in microporous scaffolds [61, 62]. Studies on shallow pillar like architectures were also performed to assess the cell responses [63-65].

These studies mainly indicate the role of individual topographic elements in directing cell behaviors. However, since in vivo environments represent a complex mélange of diverse cues varying in geometry, regularity and size, these studies offer a limited scope in understanding the synergic contribution from diverse topographic elements in the cell niches. Hence it is important 
to include the hMSC behavioral responses with topographic signals in multiple topographical combinations. There has been a limited understanding of combined effects of the surface chemical and topographic cues in directing the adhesion, morphology and fates of hMSCs. Along with the nanoscale roughness induced by surface chemical modification, considering the presence of both micro and nano scale topographies prevalent in native cell environments, we aimed to further introduce hole/pillar/groove micro-topographical structures. Since most of topographical effects are also limited to two-dimensional (2D) mat like patterned features, we found the necessity to further include hole/pillar based structures which can provide a semi $2 \mathrm{D}$ and $3 \mathrm{D}$ culture environment during hMSC expansion and differentiation.

Morphological evaluation of the adhered hMSCs revealed a well spread morphology and adhesion of cells on the H-P-G, H-P and flat PDMS substrates. Previously, the surface chemical modification and stable ECM protein layers on flat PDMS were found to enhance cell spreading as also observed in our control substrate (chemically modified Flat PDMS (Figure 6-1 c)). However, since the spreading of cells is linked to the formation of multiple focal adhesion points, recent studies on grooved based structures have also reported a higher cell spread area [59]. Hence the surface topography could play an active role in stabilizing cell adhesion and formation of uniform cell layers with high cell-cell contacts especially on the groove based H-P-G substrates (Figure 6-2 a). Although, the H-P substrates showed higher cell coverage compared to flat-PDMS, slight inconsistencies were observed in terms of overall cell sheet coverage perhaps due to the higher cell density observed around the cylindrical pillars (Figure 6-2 b). Detailed observation of cell layers revealed specific cell sheet morphological details (Figure 6-3 a, b), topographic preferences and filipodial anchorage points around individual topographic elements (Figure 6-2 c and Figure 6-3c) which could have synergistically influenced the resultant behaviors such as cell adhesion, proliferation and cell sheet formation.

Quantification of cell adhesion revealed a relatively higher cell adhesion on both H-P-G and H-P substrates compared to flat substrates (Figure 6-4). This reveals the high reliance and preference 
towards topographic elements compared to flat substrates to form anchorage (adhesion) points which are consistent with the morphology (Figure 6-2) and cell clinging trends (Figure 6-3) observed on these substrates. Since focal adhesion affect long term cell viability, a resultant higher trend in proliferation in H-P-G and H-P substrate is observed (Figure 6-5) wherein H-P-G substrates showed an increasing trend in from Day 7. Although the initial adhesion was noted as significantly high for H-P-G substrates on Day 1, the initial (Day 3) proliferation was lower than H-P substrates and almost comparable with the Flat PDMS values. This could be attributed to the relatively sparse cell distribution around the pillar/grooves and almost uniform cell distribution throughout the sample with some cells attached to the surface grooves. However, on the H-P substrates, there was already a high colonization of cell around the pillars and hence a high cellcell contact at the vicinity of the pillars. Since the establishment of cell-cell contact highly desirable for high hMSC proliferation and ECM secretion by PI3K signaling, relatively higher degree of initial proliferation was observed on H-P substrates [66]. However, the H-P-G substrate showed enhanced surface coverage and high cell-cell contact formation on stable cell sheet after day 7 (Figure 6-2) and hence after day 7 and until day 14, they showed a growing trend with significantly higher proliferation compared to all substrates. 


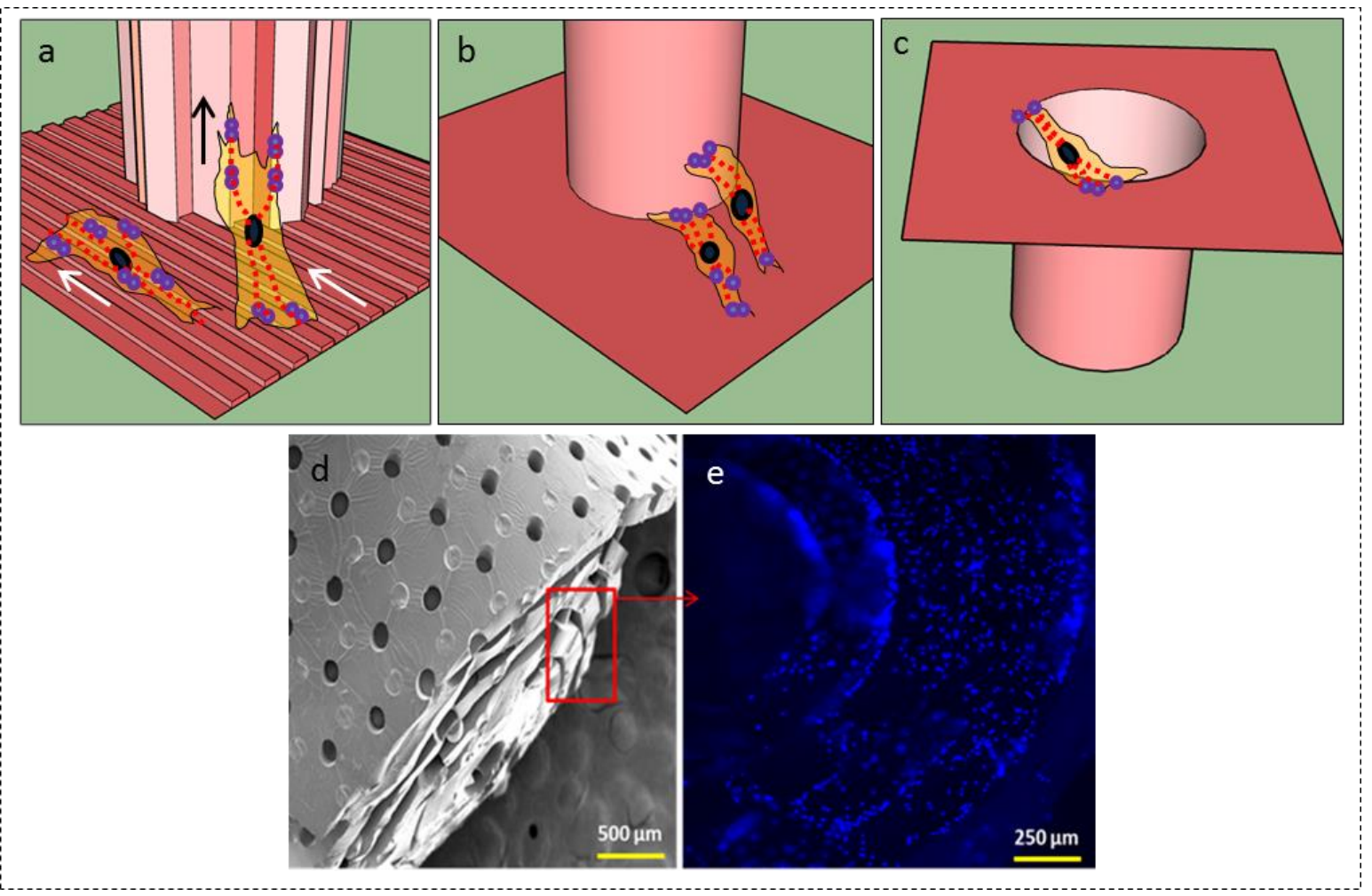

Figure 6-7: Illustration of mechanisms of hMSC adhesion and cytoskeletal filament organization in response to individual topographic elements: Cell morphology around (a) ridged pillars and grooves (b) cylindrical pillars and (c) holes. (d, e) SEM and cross sectional nuclear staining (DAPI) on the rolled hole/pillar substrates as a 3D tissue construct. The black and white arrows indicate the actin filament direction on the ridges/grooves on the ridged pillar and patterned 2D platform respectively

The observed cell adhesion and proliferation trends in response to individual physical features could be summarized as below (Figure 6-7). Since hMSC cytoskeletal activation is linked to the nature of trans-

membranal integrin triggered focal adhesion formation which are highly sensitive to microscale topography, distinct morphological endpoints (as seen in Figure 6-2 and Figure 6-3) could be observed. The ridges (elevations) on the surface and the pillars of the H-P-G provide anchorage points for the cells wherein integrin binding and recruitment of focal adhesion associated fibers and the resultant stresses can be relatively high compared to groove regions or the flat substrates [67-69]. The cells sense the ridges and showed a highly spread profile while anchoring at these 
sites (Figure 6-7 a). Within 2-20 $\mu \mathrm{m}$ paths, there is high tendency of integrin binding and actin polymerization on the grooved/ridged supports which promote orientation and regulation of hMSC fates by Wnt signaling [33, 59, 70, 71]. This phenomenon may result in stronger adhesion $[71,72]$ and subsequently higher proliferation [58], migration [58], cell sheet formation [59] and differentiation [59, 73]. The absence of groove/ridged features in H-P substrates may have influenced the higher preference of hMSC colonization at the pillars, however the absence of ridged structures on pillars or the base may have resulted in lower spreading and weaker anchorages at the clinging sites (Figure 6-7 b). These factors could have influenced the formation of relatively inconsistent and weaker cell sheet formation on these substrates. It was also noted that the cells adhered to the pillar structures in H-P substrates only up to a certain (one-third) pillar height which is almost the height of the ridged pillar in H-P-G substrates. The hole features also played a role in guiding cell adhesion since the edge of the holes were used as clinging sites by the cells with a well-spread morphology. The stable cell coverage could also be contributed by the holes due to a higher cell anchorage and spreading promoted at the perimeter of the holes (Figure 6-7 c). This could result in enhanced cytoskeletal tension and since hMSCs at the holes can access the expansion/differentiation medium in 3D like niche [61], which can synergistically contribute for enhanced cell-microenvironment interactions and differentiation. Nevertheless, these contributing factors which influenced stable cell formation in micropatterned substrates could also significantly influence the differentiation of hMSCs to osteogenic lineage. Naturally, the adhesion profiles and close cell-cell contact could influence higher collagen synthesis in the ECM which is a desirable factor for healthy osteogenesis and mineralization. From the alizarin-red staining for calcium biomineralization, a healthy osteogenesis and stable cell sheet formation could be seen even on day 21 on H-P-G and H-P substrates (Figure 6-6 a). Although the chemically modified flat PDMS showed a high cell adhesion compared to unmodified PDMS, slight peeling-off of cell layers towards day 21 was observed, which shows the strong influence of micro-topography in healthy cell anchorage and stable cell sheet formation. Further, the gene expression comparison 
between the H-P-G and H-P substrates could provide a quantitate fold based estimate, in which the influence of grooves and ridged pillars could be clearly linked to positively influence the expression of osteogenic biomarkers on hMSCs adhered on H-P-G substrates. Since past studies have individually ascertained the positive influence of holes, pillars and grooves on osteogenic lineage regulation of hMSCs [33], the H-P-G substrate could demonstrate positive synergistic influence of topographic features in promoting osteogenesis in semi $2 \mathrm{D}$ or $3 \mathrm{D}$ bone/cartilage tissue like niches.

Taken together, we demonstrate an easily fabricable, reproducible and adaptable PDMS based model to study the combinatorial influences of physical features and surface chemical modification on the morphology, adhesion, viability and osteogenic potency of hMSCs. Since a thin membranous soft/hard layers of cell laden materials are desired in several tissue engineering applications, similar fabrications with diverse range of materials (E.g., alginate, agarose), varying in texture or chemical composition could be readily applied for bone, cartilage, retinal/corneal and dental tissue regeneration. Although cell penetration into similar hole/pillar based stacked architecture of PDMS structures have been shown previously [74], the implementation of such structures in tissue engineering studies has not been considered. Existing 3D printing techniques are expensive, require laborious pre-material preparations and are prone to limitations while creating stable and high-resolution features with good repeatability at sizes below 20-30 $\mu \mathrm{m}[75$, 76]. Hence the dynamic adaptability of PDMS could be utilized in systems wherein the hole/pillar based sheets could be either stacked/rolled to mimic tissue like architectures (Illustration in Figure 6-7d, e). A major advantage of our platform is the easy tenability of feature sizes, mechanical properties and topography which can be controlled to elicit specific cell-surface interactions applicable in cell/tissue specific studies. Such considerations would also be advantageous and readily applicable in organotypic models requiring a realistic mimicking of surface chemical and topographical cell environments. 


\subsubsection{Conclusion}

In vivo tissue environments regulate cell behaviors through a complex mélange of biochemical and biophysical signals. Conventional in vitro studies concerning stem cell-substrate offer a limited understanding of comprehensive effects of ECM properties on cell behaviors. To study the combinatorial role of various ECM elements present in natural niches, we fabricated 3D like hole/pillar/groove/ride based PDMS substrates which were subjected to surface chemical modification for Col1 protein immobilization. We report distinct differences in cell morphology and adhesion in response to underlying topography which could subsequently influence cell proliferation, cell-sheet formation and osteogenic differentiation. The H-P-G substrates demonstrated highest stabilization of cell sheet formation and highest expression of osteogenic biomarkers which reveals the dominant contribution of a combination of topographical elements to synergistically influence key hMSC behaviors. We present an easily fabricable, reproducible and adaptable system which can propel rational bio-design strategies for tissue regeneration studies, on-chip differentiation and cell behavioral regulation in complex tissue like niches and organotypic models. 


\section{References}

[1] Chuah, Y.J., et al., The effects of poly (dimethylsiloxane) surface silanization on the mesenchymal stem cell fate. Biomaterials Science, 2015. 3(2): p. 383-390.

[2] Kuddannaya, S., et al., Surface Chemical Modification of Poly (dimethylsiloxane) for the Enhanced Adhesion and Proliferation of Mesenchymal Stem Cells. ACS Applied Materials \& Interfaces, 2013. 5(19): p. 9777-9784.

[3] Faia-Torres, A.B., et al., Osteogenic differentiation of human mesenchymal stem cells in the absence of osteogenic supplements: A surface-roughness gradient study. Acta Biomaterialia, 2015. 28: p. 64-75.

[4] Glueck, M., et al., Induction of Osteogenic Differentiation in Human Mesenchymal Stem Cells by Crosstalk with Osteoblasts. Bioresearch Open Access, 2015. 4(1): p. 121-130.

[5] Haeri, S.M.J., et al., Osteogenic differentiation of human adipose-derived mesenchymal stem cells on gum tragacanth hydrogel. Biologicals, 2016. 44(3): p. 123-128.

[6] Jin, L., et al., Stimulated Osteogenic Differentiation of Human Mesenchymal Stem Cells by Reduced Graphene Oxide. Journal of Nanoscience and Nanotechnology, 2015. 15(10): p. 7966-7970.

[7] Kim, S., et al., Promotion of the Osteogenic Differentiation Potential of Human Inferior Turbinate-derived Mesenchymal Stem Cells by Mechanical Memory. Tissue Engineering Part A, 2015. 21: p. S343-S344.

[8] He, W., et al., In vitro Effect of $30 \mathrm{~nm}$ Silver Nanoparticles on Adipogenic Differentiation of Human Mesenchymal Stem Cells. Journal of Biomedical Nanotechnology, 2016. 12(3): p. 525-535.

[9] Kohl, Y., et al., Effect of gold nanoparticles on adipogenic differentiation of human mesenchymal stem cells. Journal of Nanoparticle Research, 2011. 13(12): p. 6789-6803.

[10] Tenstad, E., et al., Extensive adipogenic and osteogenic differentiation of patterned human mesenchymal stem cells in a microfluidic device. Lab on a Chip, 2010. 10(11): p. 14011409.

[11] Mauney, J.R., V. Volloch, and D.L. Kaplan, Matrix-mediated retention of adipogenic differentiation potential by human adult bone marrow-derived mesenchymal stem cells during ex vivo expansion. Biomaterials, 2005. 26(31): p. 6167-6175.

[12] Pittenger, M.F., et al., Characterization of the differentiation of human mesenchymal stem cells to the chondrogenic, adipogenic and osteogenic pathways. Molecular Biology of the Cell, 1997. 8: p. 654-654.

[13] Boroujeni, S.M., et al., The synergistic effect of surface topography and sustained release of TGF-1 on myogenic differentiation of human mesenchymal stem cells. Journal of Biomedical Materials Research Part A, 2016. 104(7): p. 1610-1621.

[14] Yu, H.Y., et al., Insights into the Role of Focal Adhesion Modulation in Myogenic Differentiation of Human Mesenchymal Stem Cells. Stem Cells and Development, 2013. 22(1): p. 136-147.

[15] Liu, J.L., et al., MicroRNA 16 enhances differentiation of human bone marrow mesenchymal stem cells in a cardiac niche toward myogenic phenotypes in vitro. Life Sciences, 2012. 90(25-26): p. 1020-1026.

[16] Tian, H., et al., Myogenic differentiation of human bone marrow mesenchymal stem cells on a 3D nano fibrous scaffold for bladder tissue engineering. Biomaterials, 2010. 31(5): p. 870-877.

[17] Chen, G.P., et al., Chondrogenic differentiation of human mesenchymal stem cells cultured in a cobweb-like biodegradable scaffold. Biochemical and Biophysical Research Communications, 2004. 322(1): p. 50-55. 
[18] Cho, J.H., et al., Chondrogenic differentiation of human mesenchymal stem cells using a thermosensitive poly (N-isopropylacrylamide) and water-soluble chitosan copolymer. Biomaterials, 2004. 25(26): p. 5743-5751.

[19] Guo, L., et al., Chondrogenic differentiation of human mesenchymal stem cells on photoreactive polymer-modified surfaces. Biomaterials, 2008. 29(1): p. 23-32.

[20] Liu, K., et al., The dependence of in vivo stable ectopic chondrogenesis by human mesenchymal stem cells on chondrogenic differentiation in vitro. Biomaterials, 2008. 29(14): p. 2183-2192.

[21] Keating, A., Mesenchymal stromal cells: new directions. Cell Stem Cell, 2012. 10(6): p. 709-16.

[22] Caplan, A.I. and D. Correa, the hMSC: An Injury Drugstore. Cell Stem Cell, 2011. 9(1): p. $11-15$.

[23] Gattazzo, F., A. Urciuolo, and P. Bonaldo, Extracellular matrix: A dynamic microenvironment for stem cell niche. Biochimica Et Biophysica Acta-General Subjects, 2014. 1840(8): p. 2506-2519.

[24] Jones, D.L. and A.J. Wagers, No place like home: anatomy and function of the stem cell niche. Nature Reviews Molecular Cell Biology, 2008. 9(1): p. 11-21.

[25] Frith, J.E., et al., Tailored Integrin-Extracellular Matrix Interactions to Direct Human Mesenchymal Stem Cell Differentiation. Stem Cells and Development, 2012. 21(13): p. 2442-2456.

[26] Benoit, D.S.W., et al., Small molecule functional groups for the controlled differentiation of human mesenchymal stem cells encapsulated in poly(ethylene glycol) hydrogels. Nature materials, 2008. 7(10): p. 816-823.

[27] Philip, D., et al., Complex extracellular matrices promote tissue-specific stem cell differentiation. Stem Cells, 2005. 23(2): p. 288-296.

[28] Becerra-Bayona, S., et al., Influence of select extracellular matrix proteins on mesenchymal stem cell osteogenic commitment in three-dimensional contexts. Acta Biomaterialia, 2012. 8(12): p. 4397-4404.

[29] Chuah, Y.J., et al., Simple surface engineering of polydimethylsiloxane with polydopamine for stabilized mesenchymal stem cell adhesion and multipotency. Scientific Reports, 2015. 5.

[30] Choi, J.H., et al., Micropatterned Culture and Differentiation of Human Bone Marrow Mesenchymal Stem Cells Using a Polydimethylsiloxane Microstencil. Journal of Biomedical Nanotechnology, 2016. 12(2): p. 366-370.

[31] Kawano, T., et al., Honeycomb-shaped surface topography induces differentiation of human mesenchymal stem cells (hMSCs): uniform porous polymer scaffolds prepared by the breath figure technique. Biomaterials Science, 2014. 2(1): p. 52-56.

[32] Kurpinski, K., et al., Anisotropic mechanosensing by mesenchymal stem cells. Proceedings of the National Academy of Sciences of the United States of America, 2006. 103(44): p. 16095-16100.

[33] Metavarayuth, K., et al., Influence of Surface Topographical Cues on the Differentiation of Mesenchymal Stem Cells in vitro. ACS Biomaterials Science \& Engineering, 2016. 2(2): p. 142-151.

[34] Chuah, Y.J., et al., Combinatorial effect of substratum properties on mesenchymal stem cell sheet engineering and subsequent multi-lineage differentiation. Acta Biomater, 2015. 23: p. 52-62.

[35] Park, J.S., et al., The effect of matrix stiffness on the differentiation of mesenchymal stem cells in response to TGF-beta. Biomaterials, 2011. 32(16): p. 3921-3930.

[36] Pek, Y.S., A.C.A. Wan, and J.Y. Ying, The effect of matrix stiffness on mesenchymal stem cell differentiation in a 3D thixotropic gel. Biomaterials, 2010. 31(3): p. 385-391. 
[37] Balloni, S., et al., Effects of Titanium Surface Roughness on Mesenchymal Stem Cell Commitment and Differentiation Signaling. International Journal of Oral \& Maxillofacial Implants, 2009. 24(4): p. 627-635.

[38] Dalby, M.J., N. Gadegaard, and R.O.C. Oreffo, Harnessing nanotopography and integrinmatrix interactions to influence stem cell fate. Nature Materials, 2014. 13(6): p. 558-569.

[39] Tejeda-Montes, E., et al., Bioactive membranes for bone regeneration applications: Effect of physical and biomolecular signals on mesenchymal stem cell behavior. Acta Biomaterialia, 2014. 10(1): p. 134-141.

[40] Engel, E., et al., Mesenchymal stem cell differentiation on microstructured poly (methyl methacrylate) substrates. Ann Anat, 2009. 191(1): p. 136-44.

[41] Lee, I.C., et al., The behavior of mesenchymal stem cells on micropatterned PLLA membranes. J Biomed Mater Res A, 2009. 91(3): p. 929-38.

[42] Li, H., et al., Role of RhoA/Rho kinase signaling pathway in microgroove induced stem cell myogenic differentiation. Biointerphases, 2015. 10(2): p. 021003.

[43] Kilian, K.A., et al., Geometric cues for directing the differentiation of mesenchymal stem cells. Proc Natl Acad Sci U S A, 2010. 107(11): p. 4872-7.

[44] Zhang, W.J., et al., Studying Cancer Stem Cell Dynamics on PDMS Surfaces for Microfluidics Device Design. Scientific Reports, 2013. 3.

[45] Velve-Casquillas, G., et al., Microfluidic tools for cell biological research. Nano Today, 2010. 5(1): p. 28-47.

[46] Migliorini, E., et al., Effect of PDMS Nanopatterned Substrates on Embryonic Stem Cells Differentiation into Neuronal Lineage. Biophysical Journal, 2011. 100(3): p. 622-622.

[47] Zhang, Q. and R.H. Austin, Applications of Microfluidics in Stem Cell Biology. BioNanoScience, 2012. 2(4): p. 277-286.

[48] Yang, M.T., et al., Assaying stem cell mechanobiology on microfabricated elastomeric substrates with geometrically modulated rigidity. Nature Protocols, 2011. 6(2): p. 187213.

[49] Yang, Z., et al., Improved Mesenchymal Stem Cells Attachment and In vitro Cartilage Tissue Formation on Chitosan-Modified Poly(l-Lactide-co-Epsilon-Caprolactone) Scaffold. Tissue Engineering. Part A, 2012. 18(3-4): p. 242-251.

[50] Occhetta, P., et al., High-Throughput Microfluidic Platform for 3D Cultures of Mesenchymal Stem Cells, Towards Engineering Developmental Processes. Scientific Reports, 2015. 5.

[51] Allazetta, S. and M.P. Lutolf, Stem cell niche engineering through droplet microfluidics. Current Opinion in Biotechnology, 2015. 35: p. 86-93.

[52] Ertl, P., et al., Lab-on-a-chip technologies for stem cell analysis. Trends in Biotechnology, 2014. 32(5): p. 245-253.

[53] Huang, C., J.X. Dai, and X.A. Zhang, Environmental physical cues determine the lineage specification of mesenchymal stem cells. Biochimica Et Biophysica Acta-General Subjects, 2015. 1850(6): p. 1261-1266.

[54] Higuchi, A., et al., Physical Cues of Biomaterials Guide Stem Cell Differentiation Fate. Chemical Reviews, 2013. 113(5): p. 3297-3328.

[55] Solanki, A., et al., Controlling Differentiation of Neural Stem Cells Using Extracellular Matrix Protein Patterns. Small, 2010. 6(22): p. 2509-2513.

[56] Halldorsson, S., et al., Advantages and challenges of microfluidic cell culture in polydimethylsiloxane devices. Biosensors \& Bioelectronics, 2015. 63: p. 218-231.

[57] Charest, J.L., et al., Combined microscale mechanical topography and chemical patterns on polymer cell culture substrates. Biomaterials, 2006. 27(11): p. 2487-2494.

[58] Janson, I.A. and A.J. Putnam, Extracellular matrix elasticity and topography: Materialbased cues that affect cell function via conserved mechanisms. Journal of Biomedical Materials Research Part A, 2015. 103(3): p. 1246-1258. 
[59] Abagnale, G., et al., Surface topography enhances differentiation of mesenchymal stem cells towards osteogenic and adipogenic lineages. Biomaterials, 2015. 61: p. 316-326.

[60] Shi, X.T., et al., Stretchable and Micropatterned Membrane for Osteogenic Differentation of Stem Cells. Acs Applied Materials \& Interfaces, 2014. 6(15): p. 11915-11923.

[61] Lo, Y.P., et al., Three-dimensional spherical spatial boundary conditions differentially regulate osteogenic differentiation of mesenchymal stromal cells. Scientific Reports, 2016. 6.

[62] Fan, H., et al., Efficacy of prevascularization for segmental bone defect repair using $\beta$ tricalcium phosphate scaffold in rhesus monkey. Biomaterials, 2014. 35(26): p. 74077415.

[63] You, R., et al., Response of filopodia and lamellipodia to surface topography on micropatterned silk fibroin films. Journal of Biomedical Materials Research Part A, 2014. 102(12): p. 4206-4212.

[64] Rizwan, M., et al., Evaluation of the corneal endothelial cell monolayer formation on synthetic guttata micro-structures to study the feasibility of corneal cell therapy. Frontiers in Bioengineering and Biotechnology.

[65] Gao, X.H., et al., Regulating cell behaviors on micropillar topographies affected by interfacial energy. Rsc Advances, 2015. 5(29): p. 22916-22922.

[66] Nelson, C.M. and C.S. Chen, Cell-cell signaling by direct contact increases cell proliferation via a PI3K-dependent signal. Febs Letters, 2002. 514(2-3): p. 238-242.

[67] Vigliotti, A., R.M. McMeeking, and V.S. Deshpande, Simulation of the cytoskeletal response of cells on grooved or patterned substrates. Journal of The Royal Society Interface, 2015. 12 (105).

[68] Teixeira, A.I., et al., Epithelial contact guidance on well-defined micro- and nanostructured substrates. Journal of Cell Science, 2003. 116(10): p. 1881-1892.

[69] McMurray, R.J., et al., Surface topography regulates wnt signaling through control of primary cilia structure in mesenchymal stem cells. Scientific Reports, 2013. 3: p. 3545.

[70] McMurray, R.J., et al., Surface topography regulates wnt signaling through control of primary cilia structure in mesenchymal stem cells. Scientific Reports, 2013. 3.

[71] Ventre, M., et al., Topographic cell instructive patterns to control cell adhesion, polarization and migration. Journal of the Royal Society Interface, 2014. 11(100).

[72] Wang, T., et al., Construction of a bioactive surface with micro/nano-topography on titanium alloy by micro-milling and alkali-hydrothermal treatment. Proceedings of the Institution of Mechanical Engineers, Part H: Journal of Engineering in Medicine, 2016.

[73] Tijore, A., et al., Modulating Human Mesenchymal Stem Cell Plasticity Using Micropatterning Technique. Plos One, 2014. 9(11).

[74] Mata, A., et al., A three-dimensional scaffold with precise micro-architecture and surface micro-textures. Biomaterials, 2009. 30(27): p. 4610-4617.

[75] Chia, H.N. and B.M. Wu, Recent advances in 3D printing of biomaterials. Journal of Biological Engineering, 2015. 9.

[76] Gu, B.K., et al., 3-dimensional bioprinting for tissue engineering applications. Biomaterials Research, 2016. 20: p. 12.

$* * * * * *$

Remarks: The above chapter is related to the manuscript that was submitted to RSC-Biomaterials

Science journal for consideration to publish as a research article 


\section{Chapter. 7 Effect of substrate topography on neuronal cell behaviors}

\subsection{Geometrically mediated topographic steering of neurite behaviors and network formation}

\subsubsection{Background}

Gaining adequate control over spatial resolution of cells is a pivotal factor in constructing tissue specific functional cellular microarchitectures. The complex functions of the brain, predominantly regulated by neuronal cells are linked to their distinct complex morphology, polarity and circuitry which necessitate a detailed understanding of neuronal micro-environments for nerve tissue regeneration and development. Neurite branching and orientation are critical to establish functional, healthy cell-to-cell contacts which are necessary during all the stages of neural development and during the post-injury corrective processes [1, 2]. Advancements in micropatterning techniques have greatly enabled in vitro studies of neuronal micro-architecture and control of resultant cell behavior and morphology with high precision and reliability [3-6]. Microscale printing of cell-interactive ECM proteins such as laminin and poly-l-lysine with sub-micron resolutions are shown to support directed growth and controllable conformity of neurites [7-11]. Neurite sensing of immediate topographic microenvironment by physical guidance from microtopographical features along with the chemical cues has been reported to influence neurite growth cone navigation, both independently [12-14] and synergistically [15-17]. From the standpoint of topographically induced neuro-morphological behavior, features such as grooved surfaces, aligned fibers, micro and nanopillars and micro-cages have been found to successfully influence neurite guidance and orientation [13, 17-20]. These studies indicate an active interaction of cells and processes with topographical features of sizes in the range of 1-10 $\mu \mathrm{m}$. Specific arrangements of 
such spacing and gratings at the cell vicinity have been shown to elicit neurite guidance and initiate growth cone direction along the predestined patterns $[9,13,21,22]$. These studies have spurred detailed investigations of neurite sensing of immediate topographical cues at diverse configurations within the close proximity of the neuronal soma. Although chemical micro-patterns are known to induce cytoplasmic re-arrangements resulting in the subsequent changes in neuritogenesis trends [23-25], the specific effects induced by the physical micro-environments in response to diverse geometrical micro-cues remain unclear.

Considering the in vivo complexity of neuronal microenvironments, it is essential to identify neuronal cell morphological responses towards diverse topographical signals from proximal cell environments. Since morphological constraints imposed on the cell body are known to affect intrinsic mechanical tensions during neuronal growth [24, 26, 27], it is interesting to know the effect of physical micro-features on neuronal development. With a need for a holistic understanding on these lines, it is imperative to consider the influence of submicron features in diverse arrangements of the topographic contact points at the vicinity of the neuronal soma with precise and quantifiable neurite morphological characterization at the levels of both single neuron and minimalistic neuronal networks. Furthermore, most of the studies in this realm do not substantially explain the intricacies of physical guidance induced by angled/curved micro-cues at the somal contact points and their eventual influence on the neuronal branching and overall directionality in regulated networks.

In this work, we have focused on these research gaps by investigating neurite outgrowth and network dynamics on PDMS micro-trap arrays encompassing a wide range of geometrical configurations (Figure 7-1). Specifically, we have patterned micro-traps of height $\sim 4.5 \mu \mathrm{m}$ of various shapes separated by micro-gaps $(\sim 2 \mu \mathrm{m}$ width) to evaluate the influence of curved and angled physical cues on neurite exit/pathfinding mechanisms. Foreseeing the effects of diverse cue angles on the growth cone guidance, we have studied the preferential trends of both entrapped and the adjacent surrounding neurons. Further, the propensity of neurites to sense the immediate 
micro-gaps and synergistically form directionally aligned neuronal networks with distinct branching and orthogonal/ oblique orientations on the substrate is reported.

Taken together, we report the influence of geometrically diverse surface topographical features on primary branching of neurites from soma and their eventual effect on regularity of network formation in an in vitro micro-array setup. Since topographic regulation at close proximity of soma which affects neuritogenesis and networking behaviors is sparsely understood, in this work we saw a need to reconstruct various complex topographical and physical scenarios. Our findings are aimed to foster potential rational strategies applicable for neural guidance and repair with a realistic outlook of complex in vivo scenarios which morphologically regulate key neuronal behaviors. The study could further aid towards creating model minimalistic networks with distinct synaptic and networking conformations which could support drug screening, electrophysiological studies and disease modelling applications. 


\subsubsection{Results}

\subsubsection{Design of the Micro-trap array}

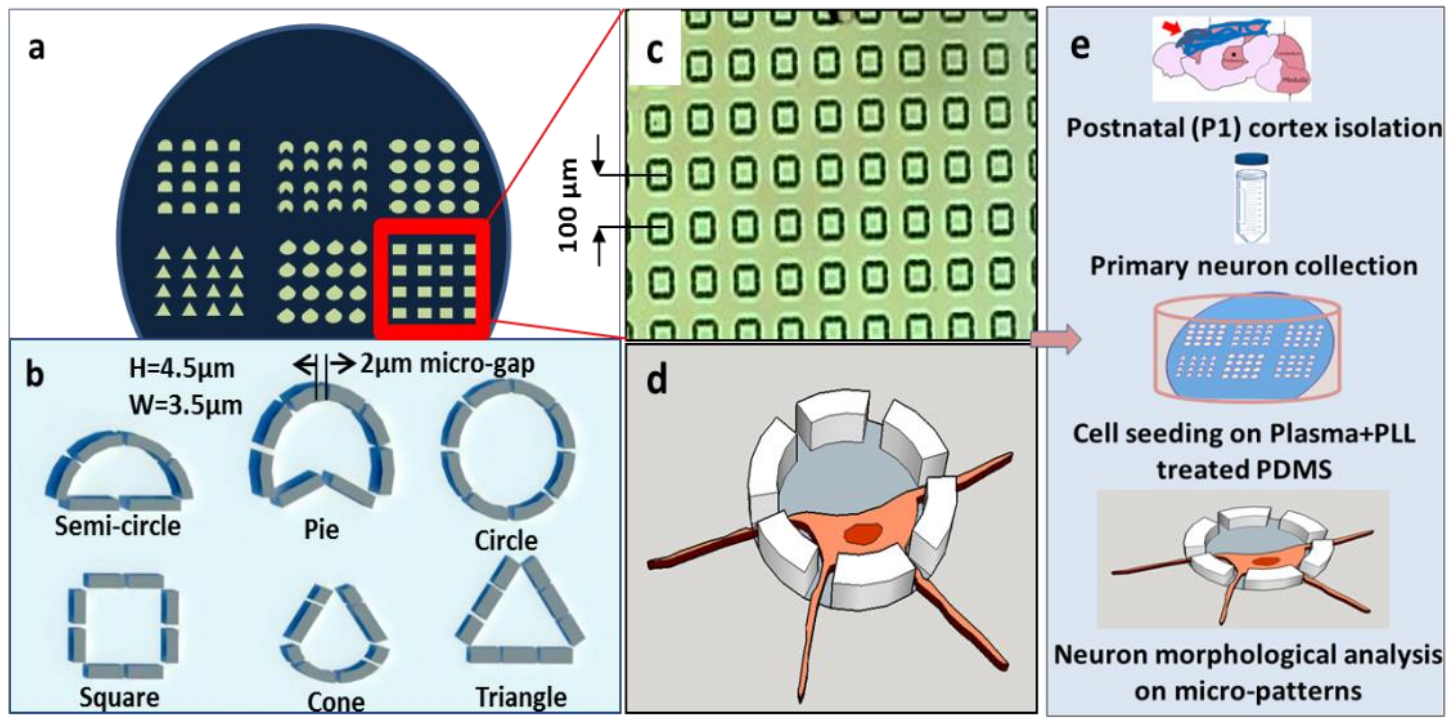

Figure 7-1: Silicon wafer with micro-trap geometrical array topographical features fabricated by negative photolithography (b) Resultant microtrap designs patterned on PDMS substrates with micro-pillar and micro-trap features. (c) Bright-field image of an individual (square) microtrap array. (d) Representative 3D illustration of neuronal cell entrapment within the microtrap and neurite extensions through the micro-gaps. (e) Schematic representation of cortical neuron isolation and culture on the surface functionalized PDMS micro-trap array substrates.

We fabricated a cyto-compatible PDMS substrate with an array of geometrically distinct microtraps encompassing varied angled and curved features from a silicon master wafer by negative photolithography (Figure 7-1 a). Each micro-trap consists of micro pillars with height of $4.5 \mu \mathrm{m}$ and width of $3.5 \mu \mathrm{m}$ arranged around a central zone for neurons to reside in and microgaps to allow the extension of neurites out of the micro-trap (Figure 7-1 b). The design considerations were determined to fulfil four specific requirements: (i) Optimal micro-gap width to guide individual neurite extensions; (ii) Optimal space (Figure 7-1c) for the neurites to freely navigate, connect and interact with the surrounding trapped/un-trapped neurons; (iii) Optimal height $(\sim 4.5 \mu \mathrm{m})$ and trap area (average trap area $\left.=70 \mu \mathrm{m}^{2}\right)$ to house single cells, minimize escape 
of trapped cells after direct cell seeding and to provide optimal space for neuronal soma (Figure 7-1 (b, d)); and (iv) Highly stable cyto-compatible substrate to withstand the duration of the culture.

\subsubsection{Neuronal growth and neurite deflection analysis}

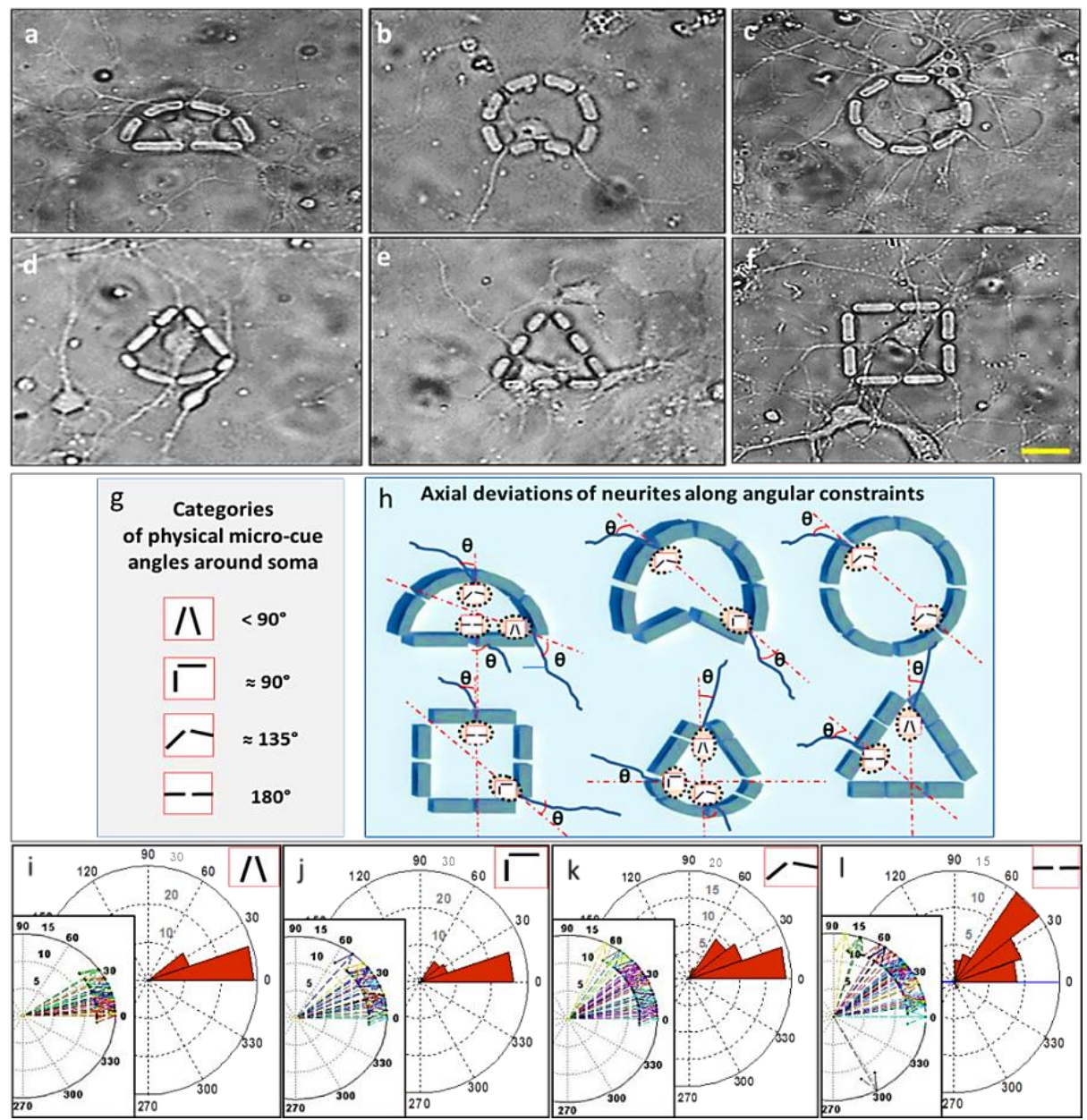

Figure 7-2: (a-f) Neuronal cell entrapment and neurite guidance through the micro-traps in various geometries. (g) Categories of neuronal soma positions based on angular arrangement of bordering microtrap cues (h) possible orientations of neuronal soma and neurite exit points at the micro-gaps. The red lines indicate various reference axes to measure neurite angles ( $\theta$ ) (i-l) Compass plots and polar plots (inset) indicating the neurite deflections from the microtrap direction (axes) at the vicinity of angled and curved profiles in various geometries. Scale bar: $20 \mu \mathrm{m}$ 
The bright field images collected on day 7 of in vitro neuron culture showed the optimal capture of healthy and mature neurons within the micro-traps of various geometries (Figure 7-2 a-f). A clear guidance of most of the neurites through the micro-gaps and the contact of the neuronal soma along the inner edges of micro-pillars was observed for entrapped neurons. Cell profiles within the microtrap suggested that that the average trap area provided optimal space to house single neurons with a somal size in the range of $12-15 \mu \mathrm{m}$ upon mid to complete maturation. The entrapped neurons could be utilized to further investigate the mechanical tensions imposed by the surrounding micro-pillars in contact with the neuronal soma [21], which could have an influence on several neurite behaviors.

The collected image data were classified into four micro-cue angle categories $\left(<90^{\circ},=90^{\circ},=135^{\circ}\right.$ and $180^{\circ}$ ) based on the angle of the geometrical cues in contact/within close proximity of neuronal soma (Figure 7-2 g). Various positions of neuron soma within the micro-traps were considered to measure the axial deviations of neurites with respect to the neighboring guidance cue angles (Figure 7-2 h). The distribution of angular deflection of neurites that extend through the microgaps along a wide range of regulation angles $\left(30^{\circ}-180^{\circ}\right)$ which fall into these four categories could be visualized by a compass plot (Figure 7-2. i-1). Distinct notable trends were observed in each micro cue angle groups. With an increase in the confinement angle from acute to obtuse angles, a corresponding increase in angular deflection of neurite over a wide-angle range was observed. Hence the physical cue angled arrangements not only affect the neurite navigation direction, but also these effects were proportional to the angle values. At acute angles the neurite directions were strictly confined to a narrow deflection range (Figure 7-2. i, j). On the contrary, with an increase in micro-cue angles at the somal contact points, the neurites exhibited a higher freedom of navigation through the micro-gaps (Figure 7-2. k, 1). The above trends indicate the possibility of neurite directions being influenced by the mechanical tensions on the soma, induced by the surrounding angular morphological constraints. It is noteworthy that the neurite navigation directions after passing through the micro-gaps are influenced without any external cues such as micro-grooves or 
lines, but these deviations are morphologically predestined at the neuronal soma based on the surrounding micro-cue angular arrangements.

\subsubsection{Morphological analysis of entrapped neurons}

The variations in neurite deflection could be attributed to varied degree of cytoskeletal rearrangements based on the relative levels of freedom offered by the confining micro-cue angles. Theses morphological changes induced by the micro-cue angular stresses on the neuronal soma assessed by the beta-tubulin immunological staining and f-actin profiles were investigated to observe distinct differences in polarization of cytoskeletal elements. The changes in these profiles were predominant at the cell and micro-trap inner wall contact junctions which are the vantage points for cell anchorage and eventual maturation. 
Analysis of cell cytoskeleton and neurite branching points

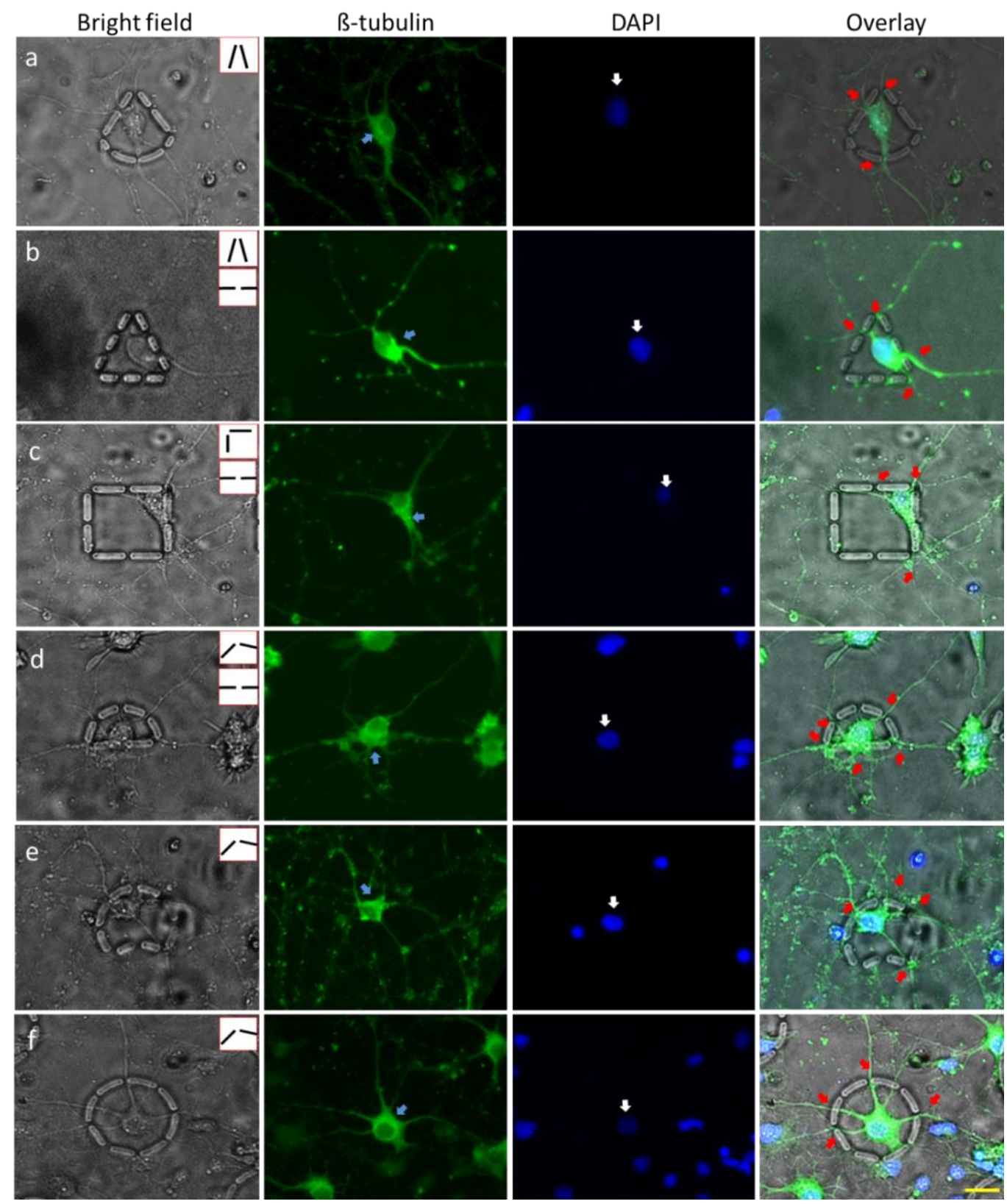

Figure 7-3: (a-f) Neuronal morphology within the angled and curved micro-traps and neurite extensions through the micro-traps. The columns indicate the bright-field, B-III-tubulin immunostaining, DAPI nuclear stain and the merged composite images of the entrapped neurons. Scale bar: $20 \mu \mathrm{m}$

Detailed morphology of entrapped neurons showed marked variations in $\beta$-tubulin polarization profiles within the neuronal soma in response to the angularity or curvature of the surrounding 
cues (Figure 7-3). Within highly angled entrapments, cytoskeletal $\beta$-tubulin was seen to be highly concentrated and polarized at the angled edges of neighboring/contacting micro-trap walls (Figure 7-3 (a-c), blue arrows). Most neurite extensions could be seen spawning from these regions (Figure 7-3, red arrows). However, within highly curved microtraps, the neurons show a wider and uniform distribution of $\beta$-tubulin all along the soma-micro-trap wall junctions, which indicates a high propensity of radial neurite branching along a widely spread surface area (Figure 7-3 (d-f), red and blue arrows). Furthermore, the shape of the nucleus appeared oval, slanted or compressed (white arrows) as per the tension on the soma imposed by angular/curved features. In general, the nuclei assumed globular profile when curved cues bordered the soma (Figure 7-3 (d-f), white arrows). However, at angular junctions, the nucleus assumed skewed orientations (Figure 7-3 (ac), white arrows). The tensions imposed by highly grooved/relaxed features are known to induce corresponding variations in nuclear morphology and tension within the nuclei [28-30]. The larger lateral surface area in contact with the soma within the curved geometries provides higher probability and freedom for the growth cone extensions along the radial pathways, compared to the relatively stricter regulation imposed by the angular cues.

\section{Analysis of F-actin profiles along angular/curved cues}

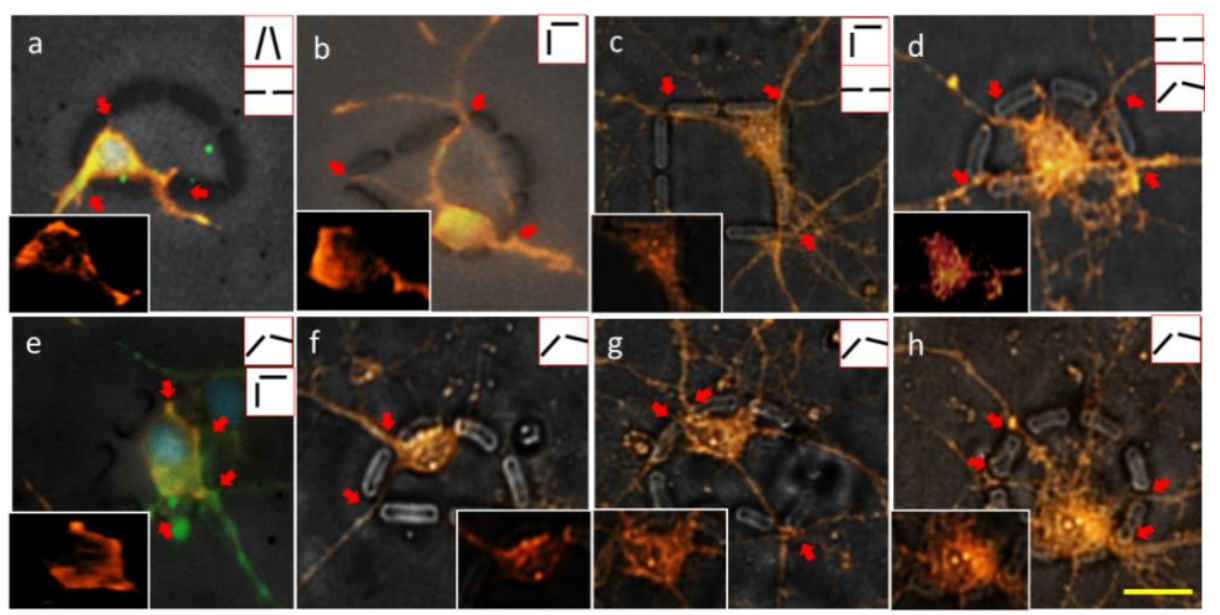

Figure 7-4: (a-h) f-actin distribution and orientation profiles along angled, cornered, flat, curved and circular profiles. Inset: Enlarged view of f-actin distribution points inside the soma. Red arrows indicate the neurite exit points through the micro-gaps. Scale bar: $20 \mu \mathrm{m}$ 
The f-actin distribution profiles indicate a tendency of growth cones to sense the nearest microgaps and steer the direction of neurites out through the micro-gap exit points. The distribution of factin shows highly concentrated polarized regions at the angular and sharp edges which in most cases are the initiation and nucleation points of for subsequent neurite outgrowth (Figure 7-4 (ac)). However, in flat and curved geometries, the f-actin is radially distributed over a larger surface area resulting in a highly spread focal adhesion profile and prominent radially directed growth cone advancements (Figure 7-4 (d-f)). The above trends indicate the restrictive control on growth cone navigation and branching dictated by the morphological changes as per the angled/curved/flat profiles neighboring the soma. 
Microtrap geometry affects neurite branching and length
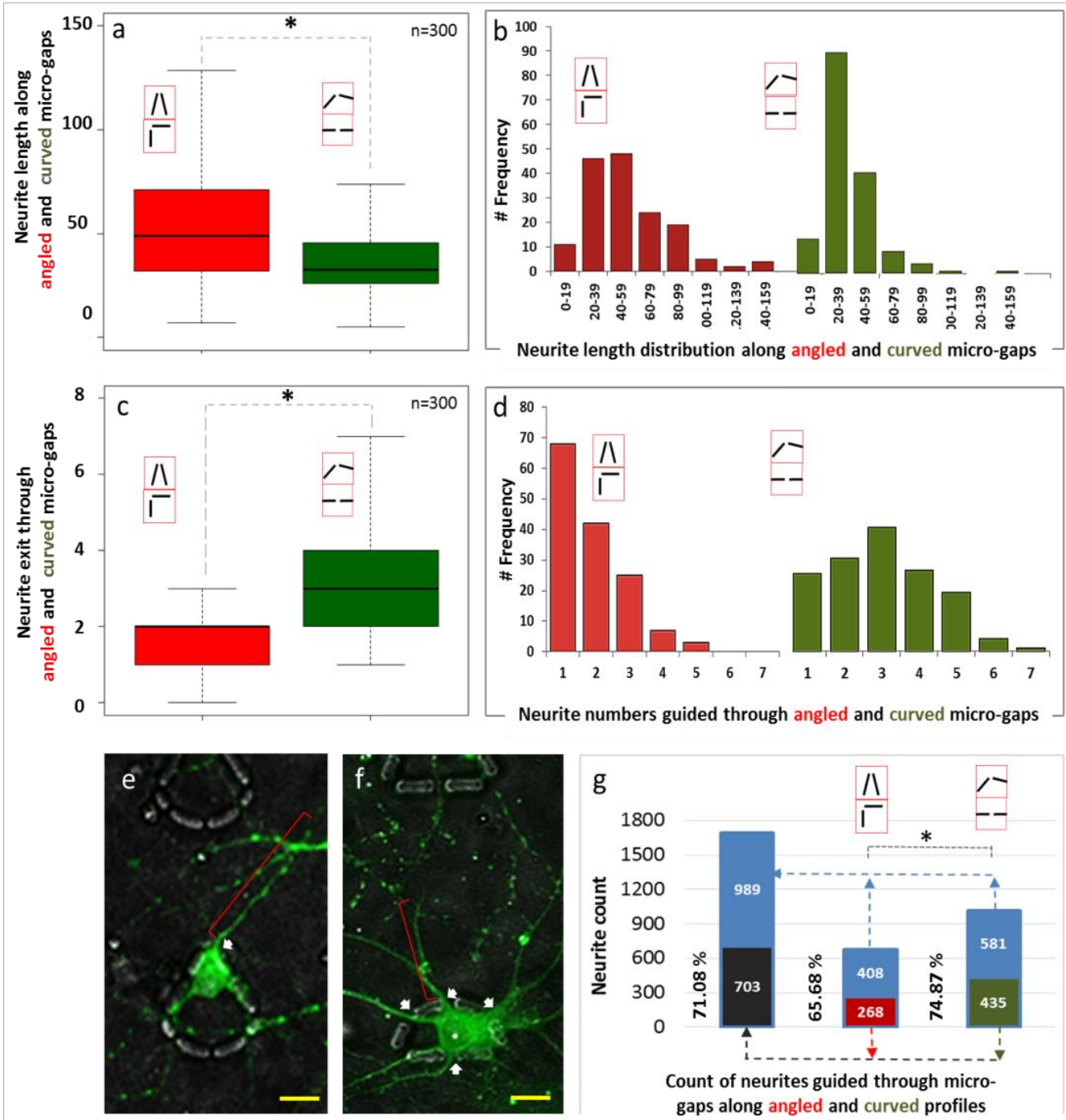

Figure 7-5: (a) Comparison of neurite lengths along angled and curved cues (b) Distribution of neurite lengths along angled/curved constraints (c) Comparison of the number of neurites exit tendency along angled and curved cues (d) Distribution of the neurite numbers which follow the exit routes through the micro-gaps along angled/curved constraints (e, f) Illustrative images of B-tubulin staining to determine morphology, precise neurite exit points and neurite length measurements in angled/curved junctions (g) Percentage wise comparison of exit and branching tendency of neurites. *indicates a p value of $<0.05$. Scale bar: $20 \mu \mathrm{m}$.

The morphological variations induced by the angled/curved/flat junctions at the soma could play a role in regulating the neurite length, branching and micro-gap exit tendencies. Neurites exited 
from the sharp/low angled cues exhibited higher mean neurite lengths compared to the curved and flat cues (Figure 7-5 a). Additionally, the neurite length distribution indicates higher tendency to form longer neurites in the highly restrictive angled junctions (Figure 7-5 b) which were explainable from the morphological analysis which indicate enhanced cytoskeletal polarization in restrictive cues. However, the curved cues showed a marked lower tendency to form longer neurites. At angular junctions, a relatively lower degree of neurite branching was observed (Figure 7-5 c) with a high tendency form only 1-4 branches per entrapped neuron (Figure 7-5c, d, e, g). In contrast, and consistent with the highly spread cytoskeletal morphology, the curved and flat cues supported enhanced branching around the neuronal soma (Figure 7-5 c, d, f, g). Irrespective of geometry of entrapment, the neurites showed a general preference (71.08\%) to sense and exit through the nearest micro-gaps (Figure 7-5 g). However, this exit trend was predominantly expressed in curved and flat cues ( $74.87 \%$ of the total exited neurites). This trend could be linked to the growth cone advancements near the micro-exit points over a broader contact area prevalent in the curved and flat cues.

\subsubsection{External guidance of neurites and the formation of minimalistic neuron networks}

In the context of formation of overall network guided by the micro-pattern array, the neurite guidance imparted on neurons outside the entrapments and the supporting glial cells were studied. 
Neurite pathfinding along external cues and the influence of glial cells
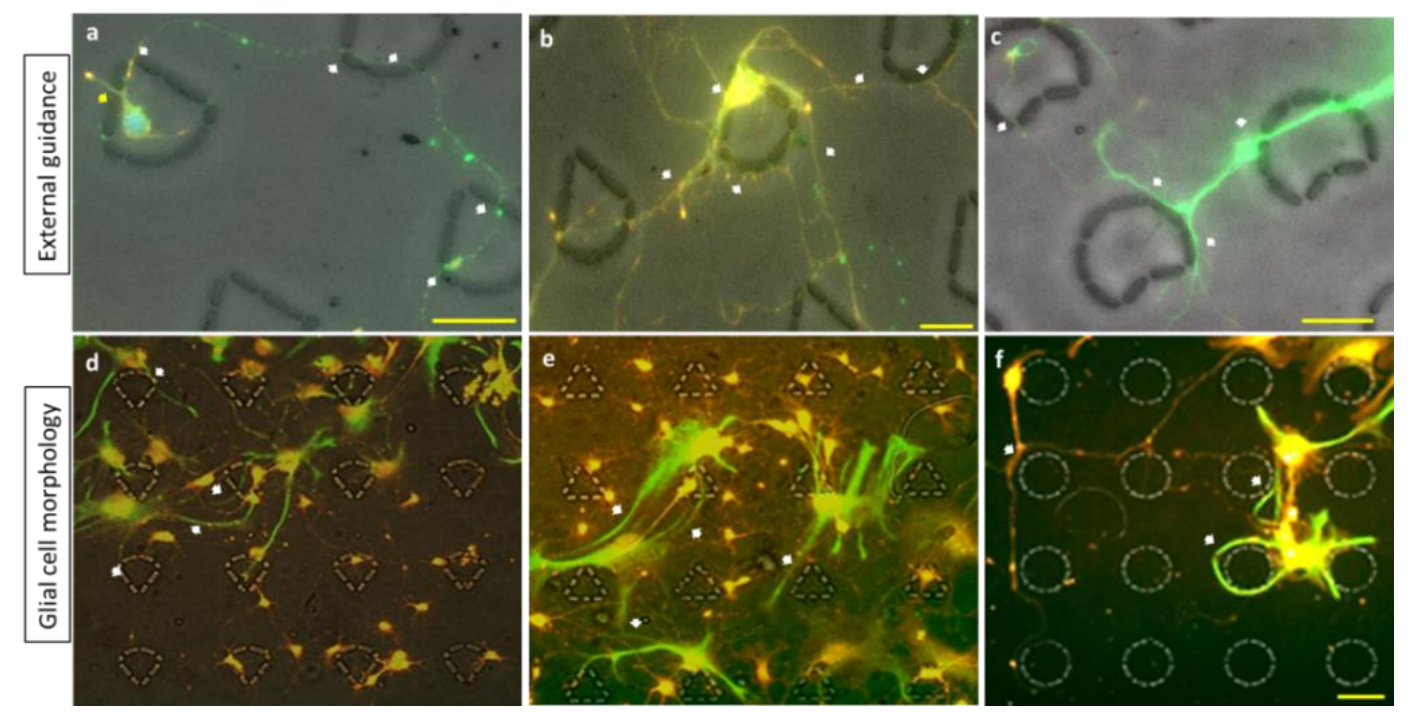

Figure 7-6: (a) Guidance of neurites through sensing the micro-gaps along the path of neurite growth cone advancement. (b and c) Neurite guidance along the external profiles of the circle, cone and pie micro-trap arrays (d-f) possible role of glial cell orientation/guidance in cone, triangle and circular microtrap arrays (white arrows indicate neurite orientation and guidance along external profiles of the geometrical shapes). Scale bar: $20 \mu \mathrm{m}$.

The neurites showed clear guidance paths around the external profile of the microtraps by either sensing the micro-gaps along the growth cone path (Figure 7-6 a) or by forming wrapped extensions around the micro-traps (Figure 7-6 b,c) and connect with the neighboring (trapped/untrapped) neurons. Additionally, along with the general neurite guidance, the glial cell pseudopods could also be clearly seen to be morphologically guided either by engulfment or external topographic guidance (Figure 7-6. (d-f)). Previous studies have also emphasized on the constructive effects of the surrounding glial cells in directing the orientation and morphology of developing neurons $[31,32]$ and show that glial cells in the proximity of neurons support long term growth and synapse formation [33-35]. Thus, the glial cell guided neuronal orientation could form additional realistic basis to understand neurite alignments especially in the context of a network of multiple neurons. 
Analysis of Minimalistic network formation on patterned substrates

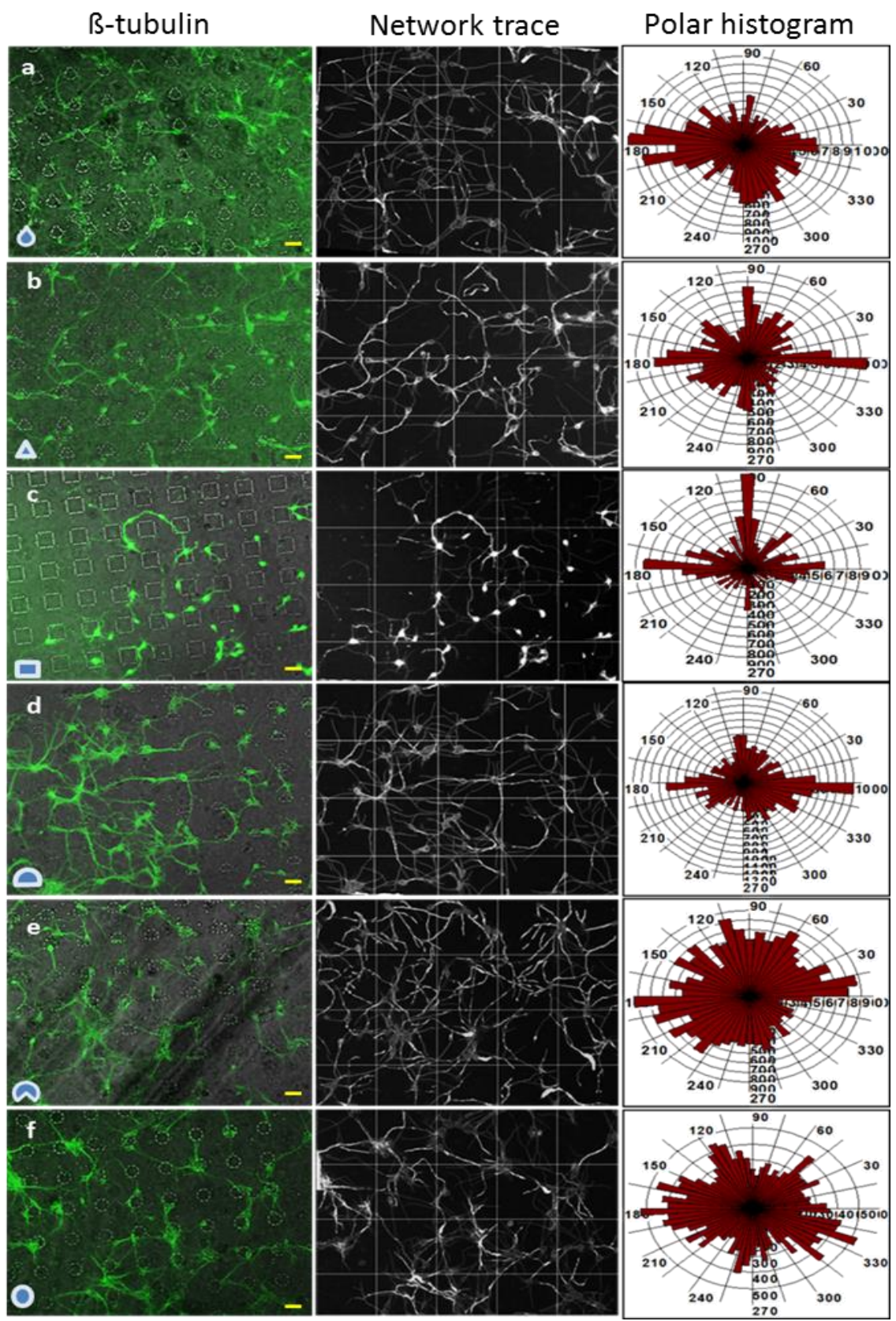

Figure 7-7: Neuronal networks formed on angled (a-c) and curved (d-f) geometric arrays. The columns indicate the B-III-tubulin stained neurons in network, corresponding high-resolution network tracing and polar histogram indicating the overall neurite orientation in each geometrical array. Scale bar: $30 \mu \mathrm{m}$. 
Generally, the neurons showed a high preference to grow in networks (Figure 7-7). However, the morphology of neurons and the directionality of the neurites extending from either the trapped or surrounding neurons were primarily determined by the underlying geometry in each micro-trap array. The overall neuron network patterns in each array showed distinguished differences in regularity and branching. The number of angled edges within a given geometry and the sharpness of the shape could play a significant role in dictating the overall orientation of neurites within the microtrap array. In highly angled arrays (cone and triangle) a general high tendency to form neurites in orthogonal directions was seen, with only a slight tendency to form neurite organization in other random directions (Figure 7-7 a, b). In square micro-traps, the neurites showed highest tendency to organize in orthogonal directions (Figure 7-7 c). Previous studies [11] [36] of neurite guidance on chemically and topographically patterned square arrays also showed a high degree of orthogonal orientation of neurites in 2-10 $\mu \mathrm{m}$ paths. Interestingly, the semi-circular patterned arrays showed a high degree of conformity along the horizontal axis. This behavior could be attributed to the influence of two sharp corners and the horizontal base of the semi-circular profile which could assist in cytoskeletal polarization and eventual neurite propagation in horizontal directions. (Figure 7-3 d, Figure 7-7 d). However, with the increase in the curvature, a noticeable decrease in neurite alignment at orthogonal directions could be observed in pie and circle arrays (Figure 7-7 e, f). On these patterns neurons generally exhibited a high degree of branching and neurite growth along random radial directions. 
Regulation of neurite directionality and length in minimalistic networks

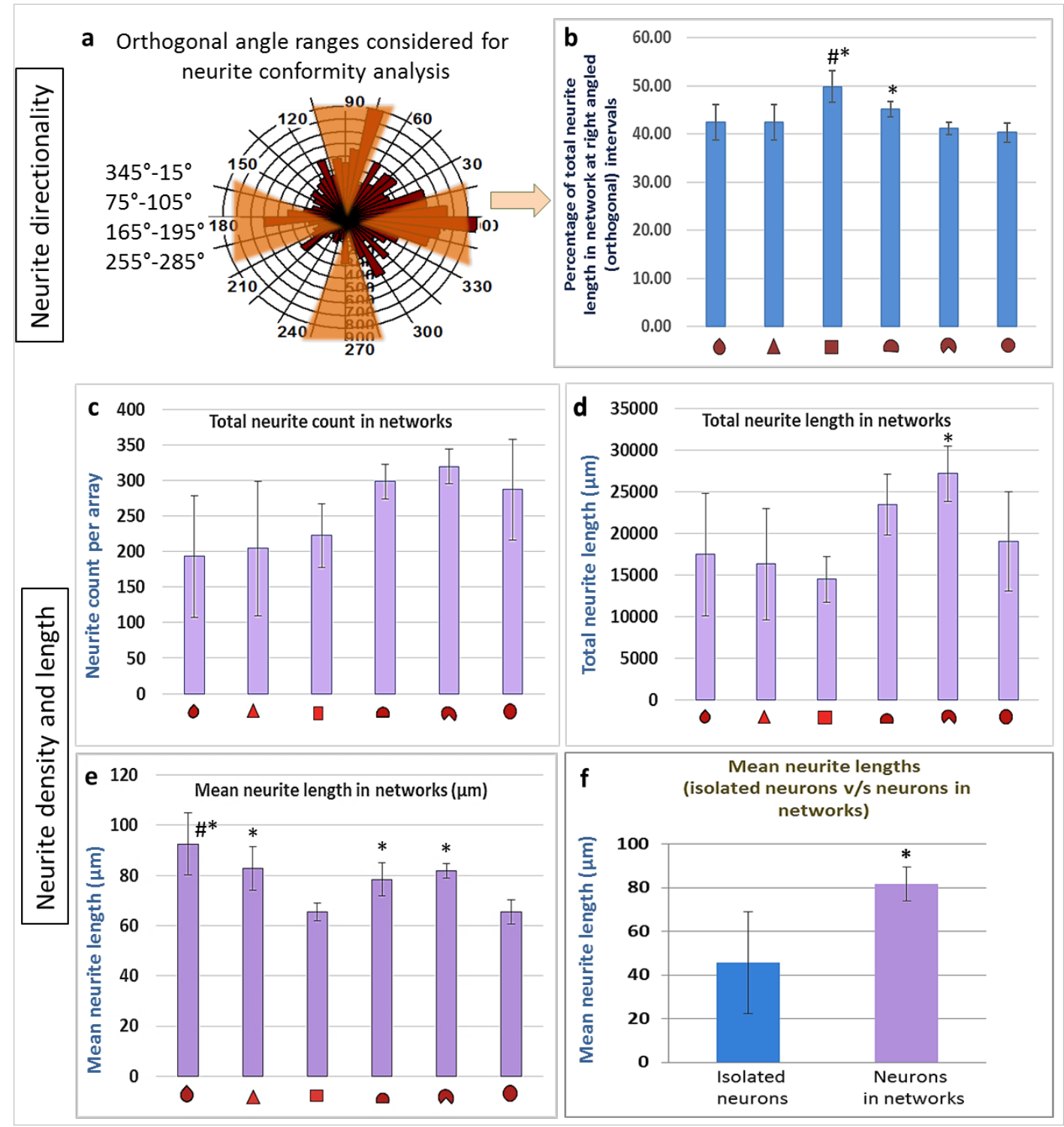

Figure 7-8: (a) Graphical representation of analysis of neurite directionality in neuronal network arrays with neurite directional commitment at orthogonal angle intervals; (b) Percentage of total neurite length in orthogonal/right angled intervals within network arrays; (c and d) Total neurite count and total (sum) of corresponding neurite lengths in geometrically varying arrays; (e) Mean neurite lengths in networks based on total neurite length and neurite density in each array; (f) Comparison of mean neurite lengths in isolated (unconnected) neurons and neurons in network arrays *indicates $p$ value $<0.05$ in comparison with circle and square micro-trap arrays. \# indicates $p$ value $<0.05$ compared to all the micro-trap arrays. 
Polar histogram and length analysis (Neurolucida Explorer, MBF Bioscience®, VT, United States) provided a quantitative assessment and statistical comparison of the overall neurite directionality and length in networks in each geometrical array. The neurite network tracings data indicated a high conformity of the neurite direction in right angled intervals $\left(345^{\circ}-15^{\circ}, 75^{\circ}-105^{\circ}\right.$, $165^{\circ}-195^{\circ}, 255^{\circ}-285^{\circ}$, Figure $7-8$ a) predominantly in the angled arrays. A relatively high percentage of total neurite in network showed orthogonal directionality in the square arrays. Although triangle and the cone arrays showed a general high tendency of orthogonal commitment, the double-sided pattern sharpness in the semicircle arrays resulted in a relatively high conformity in horizontal $\left(345^{\circ}-15^{\circ}\right.$ and $\left.165^{\circ}-195^{\circ}\right)$ directions (Figure $7-8$ b, Figure $7-7 \mathrm{~d}$ ).

The underlying topography affected both neurite density and neurite lengths in network. The highly curved arrays (circle, pie and semicircle) showed higher neurite density compared to the angled cues (square, cone and triangle) (Figure 7-8 c). This accounted for a correspondingly a higher total neurite length in network (sum of all individual neurite lengths) which showed almost similar trends as neurite density in each geometry (Figure 7-8 d). The higher neurite density in curved arrays could be linked to their pronounced tendency to initiate branching at the soma, compared to angled cues. However, the mean neurite lengths could provide more accurate picture of overall neurite length tendency per neuron based on the underlying geometry (Figure 7-8 e). The angular and sharp constraints within the cone, triangle, semicircle, and pie arrays supported higher mean neurite lengths in networks compared to the micro-trap features with higher angles/lower sharpness (square) and curved (circle) constraints. The mean length is determined from the total neurite length in networks and the neurite density. Previous studies on chemically micro-patterned designs also report the presence of longer neurites in microarray networks involving highly angled or sharp features $[21,25]$. Although a high degree of the orthogonal conformity was observed on square micro-traps, the mean neurite length in networks was significantly lower in comparison with arrays of other micro-trap shapes (Figure 7-8 b). The marked reduction in mean length in networks for square and circle patterns could be attributed to 
the absence of sharp or low angled features at regular intervals within these arrays. Further, the mean neurite lengths in networks measured significantly higher than the average neurite lengths of isolated neurons (Figure 7-8 f) which indicates the preference of neurons to grow in networks and to establish cell-cell synaptic contacts with the surrounding neurons which is essential for collective maturation of neurons [37, 38]. Previously, Hanson et.al [43] had reported similar neuronal behavior on textural guidance cues.

\subsubsection{Discussion}

We aimed to investigate the behavioral responses of cortical neurons to geometrically diverse topographical signals at the level of both single cells and neuronal networks. Past studies have extensively highlighted neurite navigation tendencies along micro-patterned chemical cues in 2-10 $\mu \mathrm{m}$ paths. These effects influence axon formation [23, 39], myelination [40], synaptogenesis [22], growth cone development $[8,14]$, and branching [9, 41-43]. Although the surface chemistry plays a significant role in regulating key neuronal behaviors [15, 44-46], considering the formidable invivo physical complexities, the inclusion of topographical elements would provide an additional basis to large scale morphological screening of neuronal niches. Thereby, the inclusion of more complex topographical and physical cue signals which affect neuronal development, morphology and polarity would certainly broaden the scope of such investigations. Although physical cues are shown to affect neurite navigation in definite micro-grooves [23, 36, 47] and micro-patterns [48], detailed mechanisms on the influence of diverse geometrical constraints around close proximity of neuronal cell body are sparsely understood. Recent studies have indicated specific cytoarchitectural polarization (of $\geq 60 \%$ of primary neurons) with high neurite navigation efficiency $[15,49,50]$ at close proximal gaps of approximately $<5 \mu \mathrm{m}$ between the cell body attachment node and the immediate topographic cues. In a previous study by Jun et.al, $2 \mu \mathrm{m}$ cues were shown to optimally guide the directional motility of growth cone in hippocampal neurons [47]. Here, we study the influence of diverse physical cue signals on neurite growth, branching, directionality and 
networking mechanisms through the variations of cytoskeletal tensions in soma in response to geometrically varying topographic cues involving $2 \mu \mathrm{m}$ micro-gaps. This system presented a diverse angular and curved arrangement of micro-cues which is a convenient tool to study neurite orientation restriction, branching and network formation in response to the nature of surrounding physical boundaries.

Firstly, we observed a high regulation of neurite outgrowth direction by sharp/angular features. In contrast, within the curved/flat features the neurites exhibited higher directional freedom and randomness of growth cone motility. A notable pronounced trend in our study is the general preference of neurite growth cones to sense and exit the micro-gaps (Figure 7-3. a-c). Most neurites $(71.08 \%)$ of the entrapped neurons showed a preferential exit through the micro-gaps. However, this tendency could be evidently influenced by angularity / curvature of surrounding physical cues. The exiting behavior was more dominant $(74.87 \%$ exit rate) along the curved $\left(\sim 135^{\circ}\right)$ and flat $\left(180^{\circ}\right)$ boundaries compared to the angled junctions (65.68\% exit rate) (Figure 7-5 g). This could be attributed to a relatively larger contact area of neuronal soma in these interfaces which allow the advancing filipodia to adequately sense the immediate exits (microgaps) along the highly spread profiles. These phenomena were understood in terms of morphological analysis of entrapped single neurons which showed a wider distribution of cytoskeletal elements ( $\beta$-tubulin and f-actin) along curved borders at the vicinity of micro-gaps (Figure 7-3, Figure 7-4). In contrast, the angular junctions strictly steered the filipodial advancement towards the angled corners, thereby restricting the branching and promoting longer neurite growth in confined paths (Figure 7-5a, b). The highly spread morphological distribution of cell cytoskeleton influenced by the higher angle (curved/flat) resulted in an enhanced degree of neurite branching tendency compared to the angled edges (Figure 7-5 c, d).

A key factor which could influence the above discussed trends could be the shape specific cytoskeletal reorganization in response to the sharpness or bluntness of physical restrictions. Immunostaining with neuron specific Beta-III-tubulin markers and staining of F-actin focal 
adhesion domains revealed distinct differences in the localization of cytoskeletal elements and filipodial extensions between angled (guidance angle $<90^{\circ}$ and $=90^{\circ}$ ), curved (guidance angle $\sim 135^{\circ}$ ) and flat (guidance angle $\sim 180^{\circ}$ ) profiles. The mechanical restriction and the resulting tension on the cytoskeletal elements could be seen as similar to previous works with neurite guidance dictated by distinct shapes of the micro-patterned cell adhesive ECM cues [23-25]. A high density of cytoskeletal elements is polarized and concentrated at the low guidance angled junctions whereas along the curved cues with higher guidance angles, the distribution of cytoskeletal elements within the soma seemed to be almost well distributed and aligned along the curved/flat contacting boundaries (Figure 7-3, Figure 7-4).

Consistent with previous studies, we observed that the cortical neurons used in this study preferred to grow in networks by immediately extending out their neurites to connect with the surrounding neurons through as dictated by their physical cues [48]. 


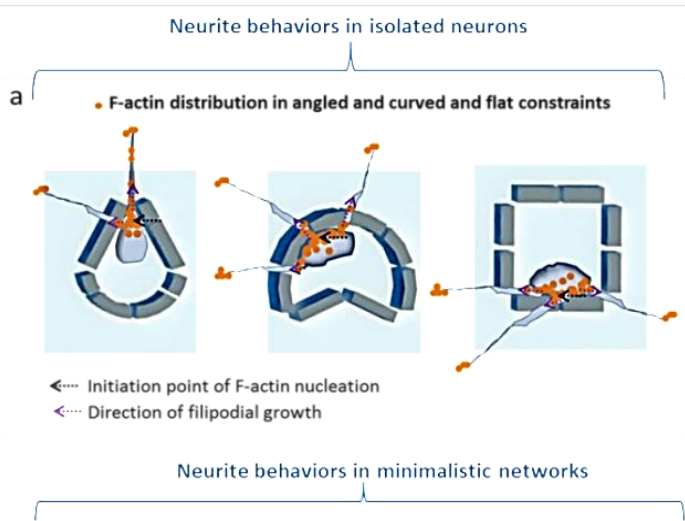

b. Higher neurite lengths in networks than in isolated neurons

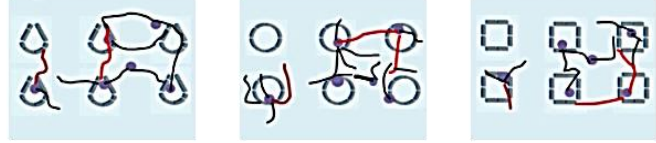

c. Neurite length and pathfinding tendency is affected by underlying microtrap array

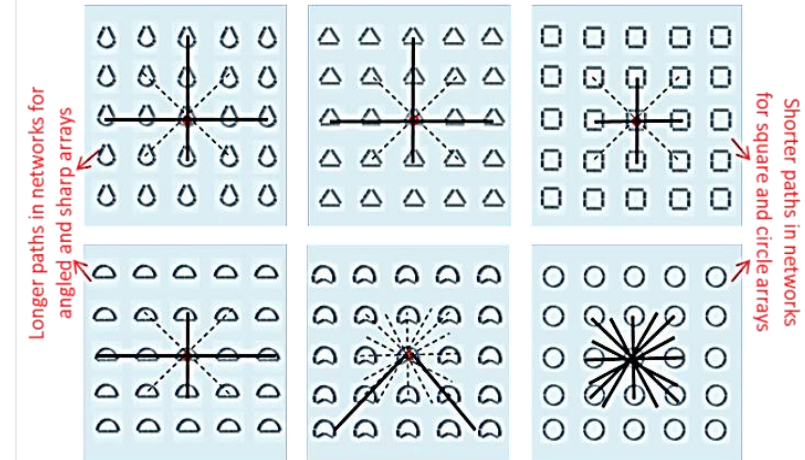

Figure 7-9: (a) Proposed mechanism of F-actin distribution and filipodial growth in response to various angular cues; (b-d) graphical illustration of neurite behaviors in minimalistic networks. The solid black line indicates preferred/dominant paths and the dotted lines indicate other potential paths in each array.

Further, distinct difference existed in the behavior of isolated single neurons and neurons in network as summarized in Figure 7-9. The neurons which preferred to grow in networks also showed a relatively higher mean neurite length in networks compared to isolated neurons (Figure 7-8 d). This indicates the formation of optimal synaptic contacts with the surrounding neurons through neurite extensions, which affects the overall growth and survival of neurons in networks. However, the neurons showed distinct preferential networking trends based on the geometric nature of the underlying micro-trap arrays. An enhanced neurite length with a relatively lower 
degree of branching was observed on sharper geometries involving at least one acute angled $(<90)$ corner (cone, triangle, semi-circle). These surfaces also promoted higher degree of orthogonal conformity. However, although square arrays showed highest orthogonal conformity, the neurons showed significantly lower neurite lengths. This could be attributed to the absence of acute angles $\left(<90^{\circ}\right)$ and also the dominant presence of flatter $\left(180^{\circ}\right)$ wall profiles which may have resulted in the neurites tending to take the shortest path to network with the surrounding neurons. The circle and circle +angled arrays showed higher dendrite count probably due to higher branching along the dominant curved boundaries. However, the mean neurite lengths in circle arrays remained low which could be attributed to the absence of highly angled features in these arrays. However, it should be noted that the overall direction and neurite navigation are also largely dependent on neurons residing outside the microtraps and the external guidance which may also be influenced by guidance from the glial cells and other external factors (Figure 7-6). The network dynamics reported in this study indicate that the filipodial transformation could be distinctly affected by the underlying geometrical physical cues and could be tuned to form more stable and well-regulated minimalistic neuronal networks with controlled neuronal branching rate, neurite length and directionality.

Based on the above discussed branching and neurite length trends, the angle/sharp cues could be seen to affect axo-dendritic polarity and higher probability of axonogenesis in orthogonal directions. Hence precise spacing and arrangement of cues could be employed in topographic platforms requiring highly controlled polarity, synaptic points and interneuron connections. Similarly, the curved/flat cues, could be utilized in developmental studies (E.g. morphogen and growth factor effects, actin dynamics and genetic screening) associated with neurite branching [51] and overall morphology $[27,52,53]$. A synchronized combination of these cues with the inclusion of nano-topographic could be used for detailed morphodynamic studies of differentiating neurons. 
The neurons of cerebral cortex are widely studied for to decipher higher brain functions and in the context of neurodegenerative diseases such as the Alzheimer's disease [54] and lafora disease [55] . Impaired growth and branching of dendrites could result in shunted neuron development, loss of neuronal polarity and synaptic plasticity. This could result in formation of aberrant or dysfunctional inter-neuronal communications and associated disorders [1, 52, 56]. Dendritic anomalies associated with Down's syndrome and Rett syndrome which occur at post-natal developmental stage are linked to variations in cell morphology [52]. In this regard, dendritic development, physiology, repair and re-organization have been closely investigated on carefully controlled micro-architectures and engineered environments [53, 57, 58]. However, most chemical environments do not represent a realistic picture and are further prone to batch variations, low resolution, unknown and nonspecific interactions. Therefore, in this study we have considered the use of simple negative lithography based facile fabrication of PDMS micro-trap arrays of varied geometries which provides a simple, tunable and re-producible system for understanding neuronal morphology and neurite dynamics influenced by diverse physical cues. These features could help in designing well organized neural circuits with controllable degree of orientation of interconnections involving dendrite-dendrite or dendrite-axon synaptogenesis. Eventually, our study could propel systematic design of neuro-responsive topographic/bio/chemical micro-environments with high resolution that would form the basis for a realistic understanding of in vivo complexities associated with neuronal development and repair mechanisms. 


\subsubsection{Conclusion}

We fabricated a simple PDMS based micro-trap array involving several geometrical variations to study the influence of micro cues on neuronal behavior in diverse angled and curved physical cue arrangements. The degree of branching, neurite pathfinding, direction and length were significantly influenced by the angularity and curvature of the physical features in the vicinity of neuronal soma. These features imposed variable degree of cytoskeletal tension within the soma, causing distinct variations in neuronal behaviors. Further, the neurons residing outside of the microtraps and the glial cells in culture also played a role in guiding the cell extensions along the external profile of the geometric patterns. These factors synergistically influenced the neurite directionality and behaviours within minimalistic networks. Large scale evaluation of neuronal response to diverse signals from the immediate physical cues would enable systematic modulation of neuronal physical and chemical microenvironments for high resolution screening of neuronal morphological variations, neuritogenesis and networking anomalies associated with several neuronal aberrations. Further, the design principles underlying topographic guidance could also be exploited in developing tunable and highly regulated nerve regeneration strategies in complex biomaterial niches. 


\section{References}

[1] Kaufmann, W.E. and H.W. Moser, Dendritic anomalies in disorders associated with mental retardation. Cereb Cortex, 2000. 10(10): p. 981-91.

[2] Lankford, K.L., S.G. Waxman, and J.D. Kocsis, Mechanisms of enhancement of neurite regeneration in vitro following a conditioning sciatic nerve lesion. J Comp Neurol, 1998. 391(1): p. 11-29.

[3] Hardelauf, H., et al., Micropatterning neuronal networks. Analyst, 2014. 139(13): p. 3256-3264.

[4] Nagamine, K., et al., Portable Micropatterns of Neuronal Cells Supported by Thin Hydrogel Films. ACS Biomaterials Science \& Engineering, 2015. 1(5): p. 329-334.

[5] Choi, J.H., et al., Micropatterning of neural stem cells and Purkinje neurons using a polydimethylsiloxane (PDMS) stencil. Lab on a Chip, 2012. 12(23): p. 5045-5050.

[6] Bani-Yaghoub, M., et al., Neurogenesis and neuronal communication on micropatterned neurochips. Biotechnol Bioeng, 2005. 92(3): p. 336-45.

[7] Turcu, F., et al., Ink-jet printing for micropattern generation of laminin for neuronal adhesion. J Neurosci Methods, 2003. 131(1-2): p. 141-8.

[8] Clark, P., S. Britland, and P. Connolly, Growth cone guidance and neuron morphology on micropatterned laminin surfaces. J Cell Sci, 1993. 105 ( Pt 1): p. 203-12.

[9] Hart, S.R., et al., Adhesive micro-line periodicity determines guidance of axonal outgrowth. Lab Chip, 2013. 13(4): p. 562-9.

[10] Xing, S., et al., Development of neurons on micropatterns reveals that growth cone responds to a sharp change of concentration of laminin. Electrophoresis, 2010. 31(18): p. 3144-51.

[11] Xu, X., et al., A patterned recombinant human IgM guides neurite outgrowth of CNS neurons. Sci Rep, 2013. 3: p. 2267.

[12] Baranes, K., et al., Topographic cues of nano-scale height direct neuronal growth pattern. Biotechnology and Bioengineering, 2012. 109(7): p. 1791-1797.

[13] Li, W., et al., Large-scale topographical screen for investigation of physical neuralguidance cues. Sci Rep, 2015. 5: p. 8644.

[14] Flanagan, L.A., et al., Neurite branching on deformable substrates. Neuroreport, 2002. 13(18): p. 2411-2415.

[15] Greene, A.C., et al., Combined chemical and topographical guidance cues for directing cytoarchitectural polarization in primary neurons. Biomaterials, 2011. 32(34): p. 8860-9.

[16] Kundu, A., et al., Superimposed topographic and chemical cues synergistically guide neurite outgrowth. Lab on a Chip, 2013. 13(15): p. 3070-3081.

[17] Millet, L.J. and M.U. Gillette, New perspectives on neuronal development via microfluidic environments. Trends Neurosci, 2012. 35(12): p. 752-61.

[18] Migliorini, E., et al., Acceleration of neuronal precursors differentiation induced by substrate nanotopography. Biotechnol Bioeng, 2011. 108(11): p. 2736-46.

[19] Bugnicourt, G., et al., Nanoscale Surface Topography Reshapes Neuronal Growth in Culture. Langmuir, 2014. 30(15): p. 4441-4449.

[20] Xie, J., et al., Electrospun nanofibers for neural tissue engineering. Nanoscale, 2010. 2(1): p. 35-44.

[21] Scott, M.A., Z.D. Wissner-Gross, and M.F. Yanik, Ultra-rapid laser protein micropatterning: screening for directed polarization of single neurons. Lab Chip, 2012. 12(12): p. 2265-76.

[22] Czondor, K., et al., Micropatterned substrates coated with neuronal adhesion molecules for high-content study of synapse formation. Nat Commun, 2013. 4: p. 2252.

[23] Tomba, C., et al., Tuning the adhesive geometry of neurons: length and polarity control. Soft Matter, 2014. 10(14): p. 2381-7. 
[24] Roth, S., et al., How Morphological Constraints Affect Axonal Polarity in Mouse Neurons. Plos One, 2012. 7(3).

[25] Jang, M.J. and Y. Nam, Geometric effect of cell adhesive polygonal micropatterns on neuritogenesis and axon guidance. J Neural Eng, 2012. 9(4): p. 046019.

[26] Grevesse, T., et al., Opposite rheological properties of neuronal microcompartments predict axonal vulnerability in brain injury. Sci Rep, 2015. 5: p. 9475.

[27] Lamoureux, P., et al., Mechanical tension can specify axonal fate in hippocampal neurons. Journal of Cell Biology, 2002. 159(3): p. 499-508.

[28] Singh, A.V., et al., Astrocytes Increase ATP Exocytosis Mediated Calcium Signaling in Response to Microgroove Structures. Scientific Reports, 2015. 5.

[29] Versaevel, M., T. Grevesse, and S. Gabriele, Spatial coordination between cell and nuclear shape within micropatterned endothelial cells. Nature Communications, 2012. 3.

[30] Versaevel, M., et al., Cell confinement: putting the squeeze on the nucleus. Soft Matter, 2013. 9(29): p. 6665-6676.

[31] Guy, A.T., et al., Neuronal development. Glycerophospholipid regulation of modalityspecific sensory axon guidance in the spinal cord. Science, 2015. 349(6251): p. 974-7.

[32] Piret, G., M.T. Perez, and C.N. Prinz, Support of Neuronal Growth Over Glial Growth and Guidance of Optic Nerve Axons by Vertical Nanowire Arrays. ACS Appl Mater Interfaces, 2015. 7(34): p. 18944-8.

[33] Ullian, E.M., et al., Control of synapse number by glia. Science, 2001. 291(5504): p. 657-

[34] Pfrieger, F.W. and B.A. Barres, Synaptic efficacy enhanced by glial cells in vitro. Science, 1997. 277(5332): p. 1684-7.

[35] Yang, Y., et al., Contribution of astrocytes to hippocampal long-term potentiation through release of D-serine. Proc Natl Acad Sci U S A, 2003. 100(25): p. 15194-9.

[36] Dowell-Mesfin, N.M., et al., Topographically modified surfaces affect orientation and growth of hippocampal neurons. J Neural Eng, 2004. 1(2): p. 78-90.

[37] Sang, Q. and S.S. Tan, Contact-associated neurite outgrowth and branching of immature cortical interneurons. Cerebral Cortex, 2003. 13(6): p. 677-683.

[38] Cove, J., et al., Growth of neurites toward neurite-neurite contact sites increases synaptic clustering and secretion and is regulated by synaptic activity. Cerebral Cortex, 2006. 16(1): p. 83-92.

[39] Liu, W., et al., Change of laminin density stimulates axon branching via growth cone myosin II-mediated adhesion. Integr Biol (Camb), 2013. 5(10): p. 1244-52.

[40] Liazoghli, D., et al., Substrate Micropatterning as a New in vitro Cell Culture System to Study Myelination. Acs Chemical Neuroscience, 2012. 3(2): p. 90-95.

[41] Francisco, H., et al., Regulation of axon guidance and extension by three-dimensional constraints. Biomaterials, 2007. 28(23): p. 3398-407.

[42] Tonazzini, I., et al., Interaction of leech neurons with topographical gratings: comparison with rodent and human neuronal lines and primary cells. Interface Focus, 2014. 4(1).

[43] Beduer, A., et al., Investigation of the Competition Between Cell/Surface and Cell/Cell Interactions During Neuronal Cell Culture on a Micro-Engineered Surface. Macromolecular Bioscience, 2013. 13(11): p. 1546-1555.

[44] Kuddannaya, S., J. Bao, and Y. Zhang, Enhanced In vitro Biocompatibility of Chemically Modified Poly(dimethylsiloxane) Surfaces for Stable Adhesion and Long-term Investigation of Brain Cerebral Cortex Cells. ACS Appl Mater Interfaces, 2015. 7(45): p. 25529-38.

[45] Ali, S.A., I.S. Pappas, and J.G. Parnavelas, Collagen type IV promotes the differentiation of neuronal progenitors and inhibits astroglial differentiation in cortical cell cultures. Brain Res Dev Brain Res, 1998. 110(1): p. 31-8. 
[46] Fereol, S., et al., Micropatterned ECM substrates reveal complementary contribution of low and high affinity ligands to neurite outgrowth. Cytoskeleton (Hoboken), 2011. 68(7): p. 373-88.

[47] Jun, S.B., et al., Low-density neuronal networks cultured using patterned poly-L-lysine on microelectrode arrays. Journal of Neuroscience Methods, 2007. 160(2): p. 317-326.

[48] Hanson, J.N., et al., Textural guidance cues for controlling process outgrowth of mammalian neurons. Lab Chip, 2009. 9(1): p. 122-31.

[49] Suzuki, I., et al., Stepwise pattern modification of neuronal network in photo-thermallyetched agarose architecture on multi-electrode array chip for individual-cell-based electrophysiological measurement. Lab Chip, 2005. 5(3): p. 241-7.

[50] Zeck, G. and P. Fromherz, Noninvasive neuroelectronic interfacing with synaptically connected snail neurons immobilized on a semiconductor chip. Proc Natl Acad Sci U S A, 2001. 98(18): p. 10457-62.

[51] Kalil, K. and E.W. Dent, Branch management: mechanisms of axon branching in the developing vertebrate CNS. Nature Reviews Neuroscience, 2014. 15(1): p. 7-18.

[52] Emoto, K., Dendrite remodeling in development and disease. Development Growth \& Differentiation, 2011. 53(3): p. 277-286.

[53] Romanova, E.V., et al., Engineering the morphology and electrophysiological parameters of cultured neurons by microfluidic surface patterning. FASEB J, 2004. 18(11): p. 12679.

[54] Bakkour, A., et al., The effects of aging and Alzheimer's disease on cerebral cortical anatomy: specificity and differential relationships with cognition. Neuroimage, 2013. 76: p. 332-44.

[55] Ortolano, S., et al., Loss of GABAergic cortical neurons underlies the neuropathology of Lafora disease. Molecular Brain, 2014. 7: p. 7-7.

[56] Ma, W.Y. and L.L. Vaccagalloway, Reduced Branching and Length of Dendrites Detected in Cervical Spinal-Cord Motoneurons of Wobbler Mouse, a Model for Inherited Motoneuron Disease. Journal of Comparative Neurology, 1991. 311(2): p. 210-222.

[57] Romanova, E.V., et al., Self-assembled monolayers of alkanethiols on gold modulate electrophysiological parameters and cellular morphology of cultured neurons. Biomaterials, 2006. 27(8): p. 1665-9.

[58] Van Meerbergen, B., et al., Peptide-functionalized microfabricated structures for improved on-chip neuronal adhesion. Conf Proc IEEE Eng Med Biol Soc, 2008. 2008: p. 1833-6.

$* * * * *$

Remarks: The above chapter is related to the manuscript that was submitted to Biomaterials journal for consideration to publish as a research article 


\section{Chapter. 8 Discussion and future works}

\subsection{Discussion}

This study aims to develop simple and versatile strategy to evaluate the influence of diverse biochemical and biophysical cues on mammalian cell behaviors. One of the major problems associated with in vitro evaluation of long term culture is the biocompatibility and tunability of surface chemical and physical features to mimic the natural cell environments. Further, the exact mechanisms and cellular effects induced by immediate micro-environments require the design of reliable platform for consistent results with realistic emulation of in vitro signals. An adaptable PDMS based system was developed to investigate diverse micro-environmental influences which regulate the fates and development of differentiating and nascent cells whose functions are highly reliant and sensitive to changes in the ECM. In this regard, the cell behaviors of hMSCs and nascent primary neurons which are associated with complex in vivo milieu are studied.

Firstly, a surface chemical modification strategy was employed to render a biocompatible surface which could address the limitation of high inherent hydrophobicity of PDMS encountered in traditional in vitro studies (Chapter 4). In many studies, the silane based SAM layer formation on the surfaces have been utilized in highly defined molecular interactions. Inspired by enhanced surface reactivity and functionality of silanes, a covalent and/or ionic interaction based crosslinking of ECM proteins was employed which resulted in a drastic decrease in PDMS surface hydrophobicity. The key behaviors of hMSCs such as focal adhesion, morphology and proliferation could be significantly affected by silane functionality/SAM layer characteristics as well as the nature and conformation of immobilized ECM proteins. A relationship between surface chemistry and (1) cell morphological regulation/adhesion and (2) long term survival and (3) was established. By characterization of surface modification, the subtle but effective influences of surface groups, silane structures and their role in regulation of cell fates (by APTMS/APTES/MPTMS silanes with/without ECM protein) was studied. Moreover, the 
silanization could be successfully implemented within PDMS micro-channels with/without Col1 ECM protein immobilization. The modification enabled both long-term cell sheet stability and osteogenesis on PDMS chip.

Secondly, the surface chemical modification was used to study the influence of biochemical signals from several ECM proteins which are important in the context of neuronal development in both natural and synthetic micro-environments (Chapter 5). In vitro models so far, provided a limited outlook on the importance of stable adhesion and long-term survival of developing neurons on in vitro platforms. Through silanization, a stable attachment of ECM proteins (FN, Col1, laminin and Poly-L-Lysine) which are critical for neuron development, long term neuronal survival, neurite growth and synaptogenesis was tested. The model enabled investigation of synergistic influences of both silanes SAM layer and bio-active ECM protein layer in controlling the cortical neuron development. Further, the surface chemistry had an influence in selectively supporting the co-survival of astro-glial cells which form a major composition of natural neuronal niches. Finally, the effect of biofunctionalization on dendrite density, length and surface morphology was characterized by immunostaining with neuro/glial-specific biomarkers and statistical image analysis. Silane based modification on PDMS micro-channels with compartmentalized structures revealed successful adhesion, distinguishable morphology and longterm survival of both neuronal and glial cells.

Thirdly, along with the surface chemical influences, the convenience of micro-patterning and rapid-prototyping of physical features on PDMS was exploited to include diverse biophysical features relevant in the morphological-modulation of hMSC behaviors (Chapter 6). Previous studies in this regard, provided isolated and limited insight with hMSC behaviors influenced by individual micron scale topographic elements within the range of 2-20 $\mu \mathrm{m}$. We studied the combinatorial effect of holes/pillars /groove based platform which were subsequently modified with covalent Col1 protein cross-linking. The platform enabled study of multiple cues which could affect hMSC cell fates in semi-2D/3D like niches. An active regulation of hMSC cell and cell 
sheet morphology towards distinct cues which eventually directed osteogenic differentiation was characterized. A higher expression of osteogenic genes and biomineralization was observed on the micro-patterned + surface modified substrates. Hence, the system represented a convenient platform and an additional basis to study influence of diverse synergy of factors which can regulate key hMSC processes.

Finally, the biophysical regulation of neuronal cell development elicited by the geometry of micro-topographical features on the overall morphology and physiological behaviors or neurons was investigated (Chapter 7). Specifically, the role of geometrically diverse physical micro-cues at a close vicinity of cortical neurons in morphologically regulating the neurite branching, direction and networking was studied. Distinct differences in neurite branching, micro-gap sensing and path-finding tendencies in response to the underlying angled and curved cues were observed. While the curved cues promoted high degree of branching, the highly angled and sharp cues demonstrated relatively lower branching with higher neurite lengths. Further, notable differences in neurite navigation and collective conformity were observed in response to the underlying topographic arrays. The micro-fabricated platform modified with plasma and poly-lysine coating, facilitated the study of diverse signals affecting neuronal behaviors.

Taken together, the cells were strongly affected by their physical and chemical neighborhood. One of the most powerful tools to control the cell-microenvironment and affect physiologically relevant cell behaviors is by microfabrication of PDMS. In this work, inherent limitations of PDMS for long term cell studies was addressed and detailed control of chemical/physical environments was employed to study various cell regulatory signals under a common platform. PDMS surface interaction characteristics were successfully altered to elucidate and study diverse cell behaviors applicable to various mammalian cell types. Mainly, by controlled surface functionalization and topography alteration, we studied the key hMSC cellular responses of therapeutic relevance namely: adhesion, proliferation and regulation of differentiation. The study is aimed guide several aspects of devising efficient biomaterial strategies at the cellular level to 
promote stem cell stimulated repair and regeneration of tissues. Similarly, diverse factors affecting the neurite morphology and network formation were investigated. The high-resolution manipulation of immediate neuronal cell topographical niches could be readily applied in neuronal morphological screening, probing neuro-developmental anomalies, neuronal guidance and repair strategies. In the following tables, a consice overview of these effects have been summarized. 
Table 2: Mesenchymal stem cell responses to surface chemical cues

\begin{tabular}{|c|c|c|c|c|c|c|}
\hline & TCP & $\begin{array}{c}\text { Group A: } \\
\text { (PDMS+Protein) }\end{array}$ & $\begin{array}{c}\text { Group B: } \\
\text { (APTES+Protein) }\end{array}$ & $\begin{array}{c}\text { Group C: } \\
\text { (APTES+GA+Protein) }\end{array}$ & $\begin{array}{c}\text { Group D: } \\
\text { APTES/APTMS/MPTMS }\end{array}$ & $\begin{array}{l}\text { Group E: (Group D } \\
\quad+\text { Protein-C1) }\end{array}$ \\
\hline \multicolumn{7}{|c|}{ Surface properties } \\
\hline Hydrophobicity & $\begin{array}{c}\text { Slightly } \\
\text { hydrophobic }\end{array}$ & Hydrophobic & Hydrophilic & Hydrophilic & Hydrophilic & Hydrophilic \\
\hline Protein Retention & Transient & Transient and weak & $\begin{array}{c}\text { Higher than Grp.A (Ionic } \\
\text { bonding) }\end{array}$ & $\begin{array}{l}\text { Highest protein retention } \\
\text { (Covalent bonding) }\end{array}$ & N/A & $\begin{array}{l}\text { Higher than Grp.A (Ionic } \\
\text { bonding) }\end{array}$ \\
\hline $\begin{array}{l}\text { Nanoscale surface } \\
\text { roughness }\end{array}$ & N/A & Moderate to high & Low & $\begin{array}{c}\text { Higher than Grp.B, reduces on } \\
\text { protein coating }\end{array}$ & $\begin{array}{c}\text { Low, remains same with } \\
\text { protein coating }\end{array}$ & $\begin{array}{l}\text { Higher than Grp.D, reduces on } \\
\text { protein coating }\end{array}$ \\
\hline \multicolumn{7}{|c|}{ Mesenchymal stem cell behaviours } \\
\hline Cell Attachment & $\checkmark \checkmark$ & $\begin{array}{l}\checkmark(\mathrm{C} 1) \\
\checkmark \checkmark(\mathrm{FN})\end{array}$ & $\begin{array}{l}\checkmark(\mathrm{C} 1) \\
\checkmark \checkmark(\mathrm{FN})\end{array}$ & $\begin{array}{l}\checkmark \checkmark(\mathrm{C} 1) \\
\checkmark \checkmark \checkmark(\mathrm{FN})\end{array}$ & $\begin{array}{l}\checkmark \text { (APTMS) } \\
\checkmark \text { (APTES) } \\
\checkmark \text { (MPTMS) }\end{array}$ & $\begin{array}{l}\checkmark \checkmark \text { (APTMS) } \\
\checkmark \checkmark \text { (APTES) } \\
\checkmark \checkmark \checkmark \text { (MPTMS) }\end{array}$ \\
\hline Cell Spread Area & $\checkmark \checkmark$ & $\begin{array}{l}\checkmark \checkmark(\mathrm{C} 1) \\
\checkmark \checkmark \checkmark(\mathrm{FN})\end{array}$ & $\begin{array}{l}\checkmark \checkmark(\mathrm{C} 1) \\
\checkmark \checkmark \checkmark(\mathrm{FN})\end{array}$ & $\begin{array}{l}\checkmark \checkmark \checkmark(\mathrm{C} 1) \\
\checkmark \checkmark \checkmark(\mathrm{FN})\end{array}$ & $\begin{array}{l}\checkmark \checkmark \text { (APTMS) } \\
\checkmark \text { (APTES) } \\
\checkmark \text { (MPTMS) }\end{array}$ & $\begin{array}{l}\checkmark \checkmark \text { (APTMS) } \\
\checkmark \checkmark \text { (APTES) } \\
\checkmark \checkmark \text { (MPTMS) }\end{array}$ \\
\hline Cell Proliferation & $\checkmark \checkmark \checkmark$ & $\begin{array}{l}\checkmark(\mathrm{C} 1) \\
\checkmark(\mathrm{FN})\end{array}$ & $\begin{array}{l}\checkmark \checkmark(\mathrm{C} 1) \\
\checkmark \checkmark \checkmark(\mathrm{FN})\end{array}$ & $\begin{array}{l}\checkmark \checkmark \checkmark(\mathrm{C} 1) \\
\checkmark \checkmark \checkmark(\mathrm{FN})\end{array}$ & $\begin{array}{l}\checkmark \checkmark \text { (APTMS) } \\
\checkmark \checkmark \text { (APTES) } \\
\checkmark \checkmark \text { (MPTMS) }\end{array}$ & $\begin{array}{l}\checkmark \checkmark \text { (APTMS) } \\
\checkmark \checkmark \text { (APTES) } \\
\checkmark \checkmark \checkmark \text { (MPTMS) }\end{array}$ \\
\hline Prolonged Cell Adhesion & $\checkmark \checkmark$ & $\mathrm{x}$ & $\checkmark \checkmark$ & $\checkmark \checkmark \checkmark$ & $\begin{array}{l}\checkmark \text { (APTMS) } \\
\checkmark \checkmark \text { (APTES) } \\
\checkmark \checkmark \text { (MPTMS) }\end{array}$ & $\begin{array}{c}\checkmark \checkmark \text { (APTMS) } \\
\checkmark \checkmark \text { (APTES) } \\
\checkmark \checkmark \checkmark \text { (MPTMS) }\end{array}$ \\
\hline Cell sheet formation & $\checkmark \checkmark$ & $\begin{array}{l}\checkmark(\mathrm{C} 1) \\
\checkmark(\mathrm{FN})\end{array}$ & $\begin{array}{l}\checkmark \checkmark(\mathrm{C} 1) \\
\checkmark \checkmark \checkmark(\mathrm{FN})\end{array}$ & $\begin{array}{l}\checkmark \checkmark \checkmark(\mathrm{C} 1) \\
\checkmark \checkmark \checkmark(\mathrm{FN})\end{array}$ & $\begin{array}{l}\checkmark \checkmark \text { (APTMS) } \\
\checkmark \checkmark \text { (APTES) } \\
\checkmark \checkmark \text { (MPTMS) }\end{array}$ & $\begin{array}{l}\checkmark \checkmark \checkmark \text { (APTMS) } \\
\checkmark \checkmark \checkmark \text { (APTES) } \\
\checkmark \checkmark \checkmark \text { (MPTMS) }\end{array}$ \\
\hline $\begin{array}{c}\text { Cell stability in } \\
\text { microfluidic channels }\end{array}$ & N/A & $\begin{array}{l}\checkmark(\mathrm{C} 1) \\
\checkmark(\mathrm{FN})\end{array}$ & $\begin{array}{l}\checkmark \checkmark(\mathrm{C} 1) \\
\checkmark \checkmark \checkmark(\mathrm{FN})\end{array}$ & $\begin{array}{l}\checkmark \checkmark \checkmark(\mathrm{C} 1) \\
\checkmark \checkmark \checkmark(\mathrm{FN})\end{array}$ & $\begin{array}{l}\checkmark \checkmark \text { (APTMS) } \\
\checkmark \checkmark \text { (APTES) } \\
\checkmark \checkmark \text { (MPTMS) }\end{array}$ & $\begin{array}{l}\checkmark \checkmark \checkmark \text { (APTMS) } \\
\checkmark \checkmark \checkmark \text { (APTES) } \\
\checkmark \checkmark \checkmark \text { (MPTMS) }\end{array}$ \\
\hline Osteogenesis & $\checkmark \checkmark$ & $\mathrm{x}$ & $\checkmark \checkmark \checkmark$ & $\checkmark \checkmark \checkmark \checkmark$ & $\begin{array}{l}\checkmark \checkmark \text { (APTMS) } \\
\checkmark \checkmark \text { (APTES) } \\
\checkmark \checkmark \text { (MPTMS) }\end{array}$ & $\begin{array}{l}\checkmark \checkmark \text { (APTMS) } \\
\checkmark \checkmark \text { (APTES) } \\
\checkmark \checkmark \checkmark \text { (MPTMS) }\end{array}$ \\
\hline
\end{tabular}


Table 3: Neuronal cell responses to surface chemical cues

\begin{tabular}{|c|c|c|c|c|c|c|c|}
\hline & TCP & $\begin{array}{c}\text { Group A: } \\
\text { PDMS-native }\end{array}$ & $\begin{array}{l}\text { Group B: } \\
\text { APTES }\end{array}$ & $\begin{array}{c}\text { Group C: } \\
(\text { APTES+FN) }\end{array}$ & $\begin{array}{c}\text { Group D: } \\
(\text { APTES+C1) }\end{array}$ & $\begin{array}{c}\text { Group E: } \\
\text { (APTES+Lam) }\end{array}$ & $\begin{array}{c}\text { Group E: } \\
\text { (APTES+PLL) }\end{array}$ \\
\hline \multicolumn{8}{|c|}{ Surface properties } \\
\hline Hydrophobicity & $\begin{array}{c}\text { Slightly } \\
\text { hydrophobic }\end{array}$ & $\begin{array}{c}\text { Highly } \\
\text { hydrophobic }\end{array}$ & $\begin{array}{c}\text { Slightly } \\
\text { Hydrophobic }\end{array}$ & Hydrophilic & Hydrophilic & Hydrophilic & Hydrophilic \\
\hline Protein Retention & Transient & $\begin{array}{l}\text { Transient and } \\
\text { weak }\end{array}$ & N/A & $\begin{array}{l}\text { Higher than Grp.A } \\
\text { (Ionic bonding) }\end{array}$ & $\begin{array}{l}\text { Higher than Grp.A } \\
\text { (Ionic bonding) }\end{array}$ & $\begin{array}{l}\text { Higher than Grp.A } \\
\text { (Ionic bonding) }\end{array}$ & $\begin{array}{l}\text { Higher than Grp.A } \\
\text { (Ionic bonding) }\end{array}$ \\
\hline $\begin{array}{l}\text { Nanoscale surface } \\
\text { roughness }\end{array}$ & N/A & $\begin{array}{l}\text { Moderate, } \\
\text { higher with } \\
\text { protein }\end{array}$ & Low & Moderate to low & Moderate to low & Moderate to low & Moderate to low \\
\hline \multicolumn{8}{|c|}{ Neuronal cell behaviours } \\
\hline $\begin{array}{l}\text { Overall cell } \\
\text { morphology }\end{array}$ & $\checkmark \checkmark$ & $\checkmark$ & $\checkmark$ & $\checkmark \checkmark \checkmark$ & $\checkmark \checkmark \checkmark$ & $\checkmark \checkmark \checkmark \checkmark$ & $\checkmark \checkmark \checkmark \checkmark$ \\
\hline Cell Viability & $\checkmark \checkmark \checkmark$ & $\checkmark$ & $\checkmark \checkmark$ & $\checkmark \checkmark \checkmark$ & $\checkmark \checkmark \checkmark$ & $\checkmark \checkmark \checkmark$ & $\checkmark \checkmark \checkmark \checkmark$ \\
\hline Mean neurite length & $\checkmark \checkmark$ & $\checkmark$ & $\checkmark$ & $\checkmark \checkmark$ & $\checkmark \checkmark$ & $\checkmark \checkmark \checkmark$ & $\checkmark \checkmark \checkmark$ \\
\hline Neurites per cell & $\checkmark \checkmark$ & $\checkmark$ & $\checkmark$ & $\checkmark \checkmark$ & $\checkmark \checkmark$ & $\checkmark \checkmark \checkmark$ & $\checkmark \checkmark \checkmark$ \\
\hline $\begin{array}{l}\text { Neuron distribution } \\
\text { with glial cells }\end{array}$ & $\checkmark \checkmark$ & $\mathrm{x}$ & $\checkmark$ & $\checkmark \checkmark$ & $\checkmark \checkmark$ & $\checkmark \checkmark \checkmark$ & $\checkmark \checkmark \checkmark \checkmark$ \\
\hline $\begin{array}{l}\text { Long term culture } \\
\text { within micro-chip }\end{array}$ & N/A & $\mathrm{x}$ & $\checkmark$ & $\checkmark \checkmark \checkmark$ & $\checkmark \checkmark \checkmark$ & $\checkmark \checkmark \checkmark$ & $\checkmark \checkmark \checkmark \checkmark$ \\
\hline
\end{tabular}


Table 4: Mesenchymal stem cell responses to biophysical cues

\begin{tabular}{|c|c|c|c|c|c|c|c|c|c|}
\hline & TCP & $\begin{array}{l}\text { Group } \\
\text { A: Flat } \\
\text { PDMS } \\
\text { (native) }\end{array}$ & $\begin{array}{l}\text { Group B: } \\
\text { Flat PDMS } \\
\text { (modified) }\end{array}$ & $\underset{\text { Hodified) }}{\text { Group C: }}$ & $\begin{array}{c}\text { Group D: } \\
\text { H-P-G } \\
\text { (modified) }\end{array}$ & $\begin{array}{l}\text { Group E: } \\
\text { Ridge } \\
\text { pillars } \\
\text { (modified) }\end{array}$ & $\begin{array}{l}\text { Group F: } \\
\text { Smooth } \\
\text { pillars } \\
\text { (modified) }\end{array}$ & $\begin{array}{l}\text { Group G: } \\
\text { Holes } \\
\text { (modified) }\end{array}$ & $\begin{array}{c}\text { Group H: } \\
\text { Grooves } \\
\text { (modified) }\end{array}$ \\
\hline \multicolumn{10}{|c|}{ Surface properties } \\
\hline $\begin{array}{l}\text { Hydrophobicit } \\
\text { y }\end{array}$ & $\begin{array}{l}\text { hydropho } \\
\text { bic }\end{array}$ & $\begin{array}{l}\text { Highly } \\
\text { hydroph } \\
\text { obic }\end{array}$ & Hydrophilic & Hydrophilic & Hydrophilic & Hydrophilid & Hydrophilic & Hydrophilic & Hydrophilic \\
\hline $\begin{array}{c}\text { Protein } \\
\text { Retention }\end{array}$ & Transient & Weak & Stable & Stable & Stable & Stable & Stable & Stable & Stable \\
\hline $\begin{array}{l}\text { Nanoscale } \\
\text { roughness }\end{array}$ & $\mathrm{N} / \mathrm{A}$ & $\begin{array}{l}\text { Moderat } \\
\text { e with } \\
\text { protein }\end{array}$ & Moderate & Moderate / low & $\begin{array}{c}\text { Moderate / } \\
\text { low }\end{array}$ & $\begin{array}{c}\text { Moderate } / \\
\text { low }\end{array}$ & $\begin{array}{c}\text { Moderate } / \\
\text { low }\end{array}$ & $(\mathrm{N} / \mathrm{A})$ & Moderate \\
\hline $\begin{array}{l}\text { F-actin } \\
\text { profiles }\end{array}$ & $\begin{array}{l}\text { Normal, } \\
\text { flat spread } \\
\text { morpholo } \\
\text { gy }\end{array}$ & $\begin{array}{l}\text { Clumpe } \\
\text { d/irregul } \\
\text { ar cells }\end{array}$ & $\begin{array}{l}\text { Flat profile, } \\
\text { high spread } \\
\text { area }\end{array}$ & $\begin{array}{l}\text { Flat profile, } \\
\text { high cell } \\
\text { density on } \\
\text { pillars than } \\
\text { flat /holes }\end{array}$ & \begin{tabular}{|c} 
Flat profile, \\
cell \\
clinging at \\
pillars + \\
uniform \\
coverage
\end{tabular} & $\begin{array}{c}\text { High cell } \\
\text { adherence } \\
\text { preference } \\
\text { to pillar } \\
\text { ridges }\end{array}$ & $\begin{array}{l}\text { High cell } \\
\text { adhesion } \\
\text { around } \\
\text { smooth } \\
\text { pillars }\end{array}$ & $\begin{array}{c}\text { Stretched } \\
\text { cytoskeletal } \\
\text { profiles at } \\
\text { pillar edges }\end{array}$ & $\begin{array}{l}\text { Flat profile \& } \\
\text { cell adhesion } \\
\text { at ridges }\end{array}$ \\
\hline $\begin{array}{l}\text { Cell sheet } \\
\text { morphology }\end{array}$ & Normal & $\begin{array}{l}\text { Irregular } \\
\text { islands } \\
\text { of cells }\end{array}$ & $\begin{array}{l}\text { Flat and } \\
\text { well spread } \\
\text { cell sheet }\end{array}$ & $\begin{array}{l}\text { Well spread } \\
\text { \& crowding } \\
\text { arnd } \\
\text { smooth } \\
\text { pillars }\end{array}$ & $\begin{array}{c}\text { Consistent } \\
\text { cell sheet } \\
\text { with good } \\
\text { cell-cell } \\
\text { contact }\end{array}$ & $\begin{array}{c}\text { Cell sheets } \\
\text { over pillars } \\
\text { \& anchor } \\
\text { pillars at } \\
\text { ridges }\end{array}$ & $\begin{array}{l}\text { 'Climbing' of } \\
\text { sheet \& dense } \\
\text { around pillars }\end{array}$ & $\begin{array}{l}\text { Covered/stret } \\
\text { ched } / \text { with } \\
\text { focal } \\
\text { adhesion }\end{array}$ & $\begin{array}{l}\text { High and } \\
\text { strong cell } \\
\text { coverage }\end{array}$ \\
\hline Adhesion & $\checkmark \checkmark$ & $\checkmark$ & $\checkmark \checkmark \checkmark$ & $\checkmark \checkmark \checkmark \checkmark$ & $\checkmark \checkmark \checkmark \checkmark \checkmark$ & \multirow{4}{*}{\multicolumn{4}{|c|}{$\begin{array}{l}\text { The individual features contribute to the overall } \\
\text { expression of cell sheets in H-P-G and H-P substrates } \\
\text { as discussed in Chapter } 6.1\end{array}$}} \\
\hline Proliferation & $\checkmark \checkmark$ & $\checkmark$ & $\checkmark \checkmark \checkmark$ & $\checkmark \checkmark \checkmark \checkmark$ & $\checkmark \checkmark \checkmark \checkmark \checkmark$ & & & & \\
\hline $\begin{array}{c}\mathrm{Ca}^{2+} \\
\text { mineralization }\end{array}$ & $\checkmark \checkmark$ & $\mathrm{x}$ & $\checkmark \checkmark \checkmark$ & $\checkmark \checkmark \checkmark \checkmark$ & $\checkmark \checkmark \checkmark \checkmark \checkmark$ & & & & \\
\hline $\begin{array}{l}\text { Osteo-gene } \\
\text { expression }\end{array}$ & $\checkmark v$ & $\mathrm{X}$ & $\checkmark \checkmark \checkmark$ & 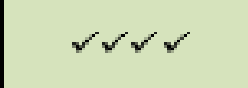 & $\checkmark \checkmark \checkmark \checkmark \checkmark$ & & & & \\
\hline
\end{tabular}


Table 5: Neuronal cell responses to biophysical cues

\begin{tabular}{|c|c|c|c|c|c|c|}
\hline & TCP & $\begin{array}{l}\text { Group A: } \\
\text { Flat PDMS }\end{array}$ & $\begin{array}{l}\text { Group A: } \\
\text { Micro cue angles } \\
\quad 90\end{array}$ & $\begin{array}{l}\text { Group B: } \\
\text { MFicro ene Ingles } \\
\Rightarrow 90\end{array}$ & $\begin{array}{l}\text { Group C: } \\
\text { Micro cue angles } \\
390\end{array}$ & $\begin{array}{l}\text { Group D: } \\
\text { Mitero cue angles } \\
>>90\end{array}$ \\
\hline $\begin{array}{l}\text { Hydrophobi } \\
\text { city }\end{array}$ & $\begin{array}{c}\text { Slightly } \\
\text { hydrophobic }\end{array}$ & $\begin{array}{c}\text { Highly } \\
\text { hydrophobic }\end{array}$ & \multirow{3}{*}{\multicolumn{4}{|c|}{$\begin{array}{l}\text { All micro-fabricated supports are hydrophilic due to plasma oxidation and PLL coating. } \\
\text { Protein retention is transient due to adsorption. Roughness>plain PDMS because of } \\
\text { plasma activation and protein corona induced variation in surface morphology }\end{array}$}} \\
\hline $\begin{array}{c}\text { Protein } \\
\text { Retention }\end{array}$ & Transient & $\begin{array}{c}\text { Transient and } \\
\text { weak }\end{array}$ & & & & \\
\hline $\begin{array}{l}\text { Nanoscale } \\
\text { roughness }\end{array}$ & N/A & $\begin{array}{l}\text { Moderate/ } \\
\text { high }\end{array}$ & & & & \\
\hline $\begin{array}{c}\text { Cell } \\
\text { cytoskeleton }\end{array}$ & $\begin{array}{l}\text { Random } \\
\text { morphology. } \\
\text { Normal } \\
\text { branching } \\
\text { /extensions }\end{array}$ & $\begin{array}{l}\text { Random } \\
\text { morphology }\end{array}$ & $\begin{array}{l}\text { Highly pointed } \\
\text { morphology at } \\
\text { sharp cues with } \\
\text { lower branching. }\end{array}$ & $\begin{array}{l}\text { Angle induced stress } \\
\text { on neurom body. } \\
\text { Low branching and } \\
\text { neurite growth at } \\
\text { comers }\end{array}$ & $\begin{array}{l}\text { Disk shaped (F- } \\
\text { actin) profiles with } \\
\text { high branching } \\
\text { tendency. }\end{array}$ & $\begin{array}{c}\text { Flat F-actin profiles } \\
\text { with tendency to form } \\
\text { branching points along } \\
\text { the linear path of soma } \\
\text { /neurite out-growth }\end{array}$ \\
\hline $\begin{array}{c}\text { Neurite } \\
\text { deflection }\end{array}$ & Random & Random & $\checkmark$ & $\checkmark \checkmark$ & $\checkmark r \checkmark$ & $\checkmark \checkmark \checkmark \checkmark$ \\
\hline $\begin{array}{l}\text { Neurite } \\
\text { branching }\end{array}$ & Random & $\begin{array}{l}\text { Random } \\
\text { /ocalized }\end{array}$ & $\checkmark$ & $\checkmark r$ & $\checkmark \checkmark r$ & $\checkmark \checkmark \checkmark \checkmark$ \\
\hline $\begin{array}{l}\text { Neurite } \\
\text { growth/lengt } \\
\text { h }\end{array}$ & \multirow{3}{*}{\multicolumn{2}{|c|}{$\begin{array}{l}\text { Depends on surface chemical } \\
\text { cues, cell density and random } \\
\text { micrónano-topographic cues. }\end{array}$}} & $\checkmark \checkmark \checkmark \checkmark$ & $\checkmark \checkmark \checkmark$ & $\checkmark \checkmark$ & $\checkmark$ \\
\hline $\begin{array}{c}\text { Neurite exit } \\
\text { tendency }\end{array}$ & & & $\checkmark$ & $\checkmark r$ & $\checkmark \checkmark \checkmark$ & $\checkmark \checkmark \checkmark \checkmark$ \\
\hline $\begin{array}{c}\text { External } \\
\text { guidance \& } \\
\text { neurite } \\
\text { network } \\
\text { morphology }\end{array}$ & & & $\begin{array}{l}\text { Highly aligned, } \\
\text { linear neurites } \\
\text { from extemal } \\
\text { neurons/glial cells. } \\
\text { High orthogonal } \\
\text { commitment }\end{array}$ & $\begin{array}{l}\text { Highly aligned, } \\
\text { morphology with } \\
\text { highest orthogonal } \\
\text { commitment (Esp. } \\
\text { Square arrayg) }\end{array}$ & $\begin{array}{c}\text { High degree of } \\
\text { branched networks } \\
\text { with lower degree of } \\
\text { orthogonal } \\
\text { commitment }\end{array}$ & $\begin{array}{l}\text { Highly aligned features } \\
\text { at angles } ~ 180^{\circ} \text { with } \\
\text { linear neurite path } \\
\text { guided by both internal } \\
\text { \& external features }\end{array}$ \\
\hline
\end{tabular}




\subsubsection{Table-summary}

In our study, a 10:1 ratio of normal PDMS was selected since it provides optimal cross-linking density between the two components when cured. An increase in the ratio of curing agent to base (i.e.,10:5- 10:3) increases the number of un-cross-linked dimethyl methylhydrogen siloxane groups, and dimethylsiloxane monomers having silicon hydride end-groups. This leaves more of the vinyl-terminated dimethylsiloxane units un-cross-linked in the elastomer. The composition of the PDMS affects the surface properties of the elastomer, because the composition determines the types of un-crosslinked and thus the lower molecular weight chains of mobile components that may reside on (or migrate to) the surface of the polymer. We observed that these differences in PDMS composition also affect the attachment and growth of cells and maintained the standard stiffness of 10:1 cross-linking throughout the surface chemical/physical modification study. The plasma modification and silanization (by APTES/APTMS/MPTMS) resulted in substantial reduction of surface hydrophobicity and improved surface reactivity. Moreover, crosslinking of FN and Col1 proteins with GA improved the cell spreading and proliferation which also aided in long term survival and osteogenic differentiation. The surfaces of hole groove and pillar based multiscale supports when modified with this chemistry resulted in further enhancement of cell sheet formation and differentiation. Neuronal cell attachment was highly supported on the silanized and laminin/PLL modified supports. The high charge interaction of stable polycationic PLL layers with neuronal membrane integrins resulted in enhanced attachment and activity of neurons in flat substrates, microfluidic channels as well as microfabricated supports. The inclusion of physical cues resulted in higher control on the overall neuron morphology and growth. On the geometrically patterned PDMS (modified with plasma+PLL), the sharper cues supported enhanced neurite growth with highly organized networks. Whereas, the curved cues support enhanced neurite branching with shunted neurite growths. These trends were also influenced by external cues and glial cells in culture 


\subsection{Limitations}

Some of the limitations of this study are enlisted below:

- Although the natural neuronal environments contain two cell types: i.e. the neurons and glial cells, the implementation of the system for co-culture between two or more cell types and dynamic long-term interactions are not studied in the context of hMSCs. This may involve cross-talks with various chemical/biomolecular components.

- Due to limited access to super resolution microscopy, the focal adhesion dynamics in terms of integrin localization was not studied. Immuno-staining of the membrane bound integrins can be used to further understand how cytoskeletal stresses from topography or the surface chemistry affect focal adhesion dynamics.

- A constant PDMS stiffness of 1:10 mixing ratio was used in the study. Since the hMSCs are sensitive to stiffness, variation in cell behaviors, the effects of varying surface hydrophobicity on cell attachment/migration could be considered to add a mechanical dimension to ECM. However, some aspects of PDMS stiffness induced changes in hMSC behaviors were discussed by a recent work (ref [74] in Chapter 2).

- Although cytoskeletal stresses are proven to be affected by the surface chemistry/surrounding topography, the extent and quantifiable degree to which these modifications affected actin-polymerization and changes in actin filament was hard to determine.

- Some of the inherent molecular mechanisms which induced morphological and its implication on electrophysiological properties of neurons could not be covered in the scope of this study. 
- Certain factors may influence protein and cell attachment on the surface: (i) defects on PDMS surface (ii) homogeneity of surface chemistry (affected by poor mixing/incomplete treatment) and (iii) low MW species migrating from the bulk and residing on the surface.

\subsection{Future directions}

In terms of cell behavior, diversity, relevance of biochemical and biophysical signals as well as characterization/fabrication methods employed in this work, several future directions are proposed:

- From this study, the cell adherence on chemically well-defined PDMS support was shown to provide a stable and robust platform to assess several cell functions with the capability to apply in microfluidic studies. Hence, co-culture between two or more cell types and dynamic long-term interactions between can be systematically investigated.

- Surface modification induces nano-scale surface roughness changes. However, to develop detailed in vitro test platforms with 3D nano-scale interactions, models involving nanofabrication of nanofibers/nano-arrays can be employed.

- As a test model to find effects of multiple cues to direct cell fate, long term culture and cell sheet formation in the context of osteogenic differentiation was studied in this work. Our model readily supported neuron viability. Since neurons showed different characteristics compared to hMSCs, the model could be used to potentially test directed differentiation of hMSCs or patient derived iPSCs to neuronal cells.

- Since PDMS based strategy involved cost-effective fabrication and simple tuning of surface properties, it could be utilized as a regular test platform to eventually design complex bio-fabrication involving expensive cell lines and materials.

- The rapid prototyping of micron scale features was used to create semi 2D/3D tissue like constructs with regular hole-pillar-groove morphology. Using this as a base substrate, A three-dimensional niche either by rolling/stacking the micro-fabricated PDMS mats could 
be employed with various stiffness level to generate models which could be used on dental/retinal/nerve tissue engineering.

- PDMS hydrophobicity could be easily tuned to obtain either flat cell sheets applicable in osteogenic models or to obtain 3D organoids/cell spheres of iPSCs or hMSCs which can be applicable in nerve/hepatic tissue engineering. Precise tuning of both hydrophobicity and micro-features can be performed to facilitate high-throughput screening of disease as well as drug conditions.

- In our study, strategies for precise control of micro-topography for neuronal morphological regulation were employed and the cell characteristics were studied with neuron specific biomarker. For further insight into direct control of substrate interactions on neuronal cell functions, models with direct electrophysiological recording of neuronal activity, monitoring of calcium influx and immunostaining with pre/post synaptic biomarkers can be implemented.

- For precise understanding of the nature of focal adhesion modulated by the underlying substrate chemistry and topography, immunostaining of proteins involved in focal adhesion clustering could be employed. Immuno-staining, high resolution imaging and statistical distribution of the membrane bound integrins, talins or vinculin proteins can provide accurate basis to correlate focal adhesion dynamics with lineage commitment or neuronal development. 


\subsection{Conclusion}

This thesis aimed to develop, simple and tunable platforms to study various cell-substrate interactions. The bone marrow and the brain environments are highly complex with diverse chemical and physical signals and molecules which affect cell functions. In this thesis, in vitro models are developed and a detailed characterization and analysis of the effect of the surface chemical and topographical/physical cues on the hMSCs and cortical neuron behaviors has been presented. To achieve this, in vitro systems based on PDMS material were built. PDMS is being used in several studies involving micro/nano-patterned synthetic bio-environments of cells and biomolecules. However, the high surface hydrophobicity and low surface reactivity of PDMS surfaces has been a critical bottleneck due to which the long-term studies of complex cell systems has not been possible.

To solve this problem and to enhance the material utility for cell studies, a silanization based chemical modification of PDMS surfaces is proposed. Specifically, functional group presenting silane molecules of distinct molecular structures such as APTES/APTMS/MPTMS was used for covalent cross-linking of ECM proteins which formed a synthetic biocompatible microenvironment for cells. The hence modified surfaces were characterized by various bio/chemical analytical techniques. Eventually the response of cells to surface chemical and topographical cues as well as the application of the platform in PDMS microfluidic devices/micropatterned surfaces was studied. One of the major improvements is the decrease in surface hydrophobicity and successful ECM protein retention. As a result, the adhesion of hMSCs was significantly improved. The hMSCs showed enhanced proliferation and differentiation into osteogenic cells over a long culture period. This confirms the applicability of this strategy for long term stem cell mediated tissue regenerative studies.

Additionally, with the inclusion of multiscale topographic elements that are relevant in the natural tissue like niches, a positive synergistic influence on hMSC behaviors has been reported. The 
chemical modification was also suitably tuned to immobilize neuron relevant ECM proteins for a stable culture and long-term examination of developing neurons isolated from neonatal rat cortex. A stable culture of cortical cells was achieved and the effect of underlying chemical cues on neuronal cell development is reported. Finally, several neuronal behaviors were assessed on both flat-modified and micro-fabricated PDMS substrates in response to diverse physical signals in the form of shape micro trap arrays. Overall, the techniques and findings are aimed to facilitate strategic design of cell instructive bio-materials and smart bio-interfaces. Further, the thesis aims to aid the investigation of the intricacies associated with cell development and differentiation in complex micro-environments. 